A COMPREHENSIVE PLANNING FRAMEWORK FOR URBAN INLAND OIL SPILL MANAGEMENT

\author{
by \\ Marija Eric \\ Master of Applied Science, Ryerson University, 2012 \\ Bachelor of Engineering, Ryerson University, 2010
}

\author{
A dissertation \\ presented to Ryerson University \\ in partial fulfillment of the \\ requirements for the degree of \\ Doctor of Philosophy \\ in the program of \\ Civil Engineering
}

Toronto, Ontario, Canada, 2019

(C) Marija Eric, 2019 


\title{
A Comprehensive Planning Framework for Urban Inland Oil Spill Management
}

\author{
Marija Eric \\ Doctor of Philosophy \\ Civil Engineering, Ryerson University, 2019
}

\begin{abstract}
Oil spills occurring on land have accounted for at least one third or over 24,000 of all land spills (approximately 76,000) of various substances reported in Ontario from 1988 to 2013. The objective of this research was to develop a comprehensive planning framework for urban inland oil spill management encompassing all three stages of spill management: (1) prevention, (2) control and (3) response. An inland oil spill database was developed and the source of each spill was analyzed. Preliminary analyses determined that approximately $46 \%$ of spills occur at fixed locations (stationary spills), $21 \%$ of spills involve moving vehicles (transportation-related), $13 \%$ involve moving vehicle accidents (transportation-related accidents) and $20 \%$ were categorized as other kinds of spills.
\end{abstract}

Sub-databases were developed for both stationary and transportation-related spills which include numeric and non-numeric data variables. Hot spot analyses (optimized version) were performed on a subset of transportation-related spills to develop a highway spill model. The highway spill model illustrates that the majority of highway spills (75\%) occur at interchanges and the remaining spills occur either on the highway ( $8 \%$ ) or at unknown locations $(17 \%)$.

A macro program was developed to simulate future spill events based on historical spill events of gasoline spills within the case study area. The variables under study were fitted with distributions and Monte Carlo or Iman Conover methods were used to generate simulation 
results spreadsheets of spill series data based on the fitted distributions. The final macro program generated 30,000 simulation results spreadsheets and compiled the results in an aggregate spreadsheet. Descriptive statistics of the numeric variables were calculated and used to recommend spill management strategies.

A simulation results spreadsheet with predicted spill records was used to develop a Geographic Information Systems (GIS) model to delineate spill pathways and calculate travel-time for overland flow and channel flow within the storm sewer system (geometric network). The model delineates the overland spill path and traces the spill path through the storm sewer network. The travel-time for each type of path is calculated and can be summed to determine the total travel-time for each predicted spill.

Keywords: inland oil spill, comprehensive planning framework, spill management, prevention, control, response, stationary, transportation-related, hot spot analysis, macro program, Monte Carlo, Iman Conover, simulation, GIS, travel-time, spill path, geometric network 


\section{Acknowledgements}

This research is funded by the Natural Sciences and Engineering Research Council of Canada. I gratefully acknowledge the help, guidance and support of Dr. James Li, Dr. Songnian Li and Dr. David Atkinson. I would also like to thank Xintao Liu and Desmond Rogan for their contribution to the development of the Web-based GIS prototype. 


\section{Table of Contents}

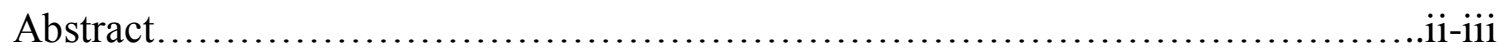

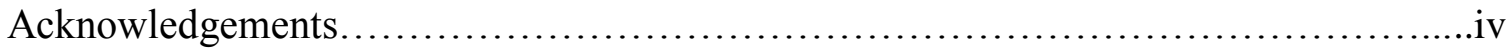

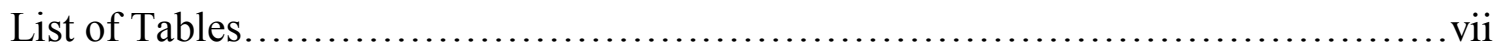

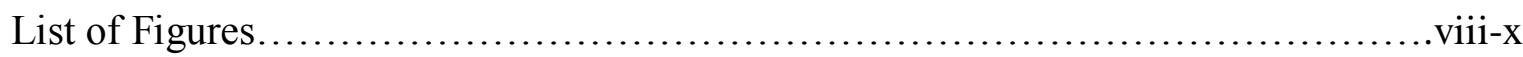

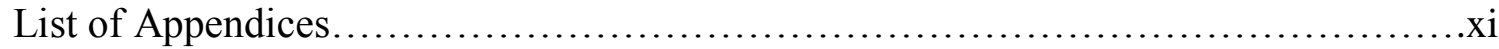

Dissertation Structure ........................................................

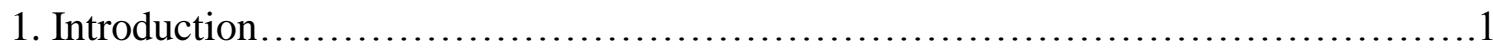

1.1 Recent Oil Spill Issues and Controversies...............................

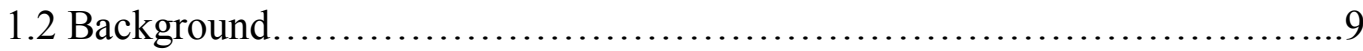

1.3 A Brief History of Oil Spills...............................................13

1.4 Government Programs and Legislation....................................16

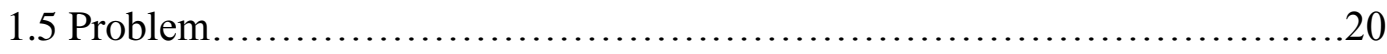

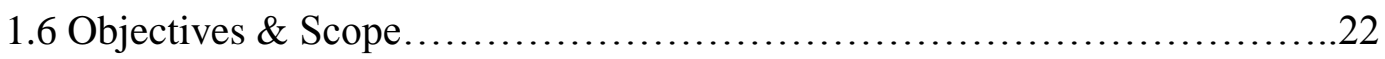

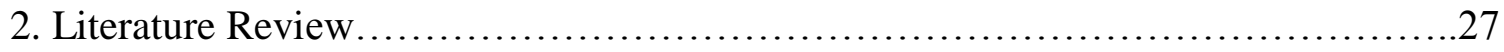

2.1 Spill Database Analysis...............................................27

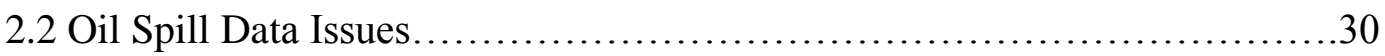

2.3 Land Spills vs. Water Spills............................................. 33

2.4 Oil Spill Impacts on Freshwater Environments.............................36

2.5 The Three-Stage Framework: Prevention, Control and Response..............41

2.6 Web-Based GIS ......................................................... 54

2.7 Spill Modelling Software..............................................60

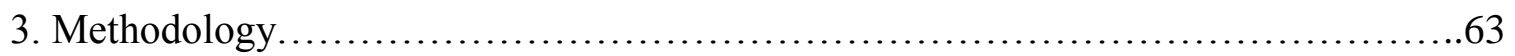

3.1 Development of a Spill Management Framework..........................63

3.2 Compilation of Spill Database \& Spill Sub-Databases......................65

3.3 Transportation-Related Spill Sub-Database \& Highway Spill Model

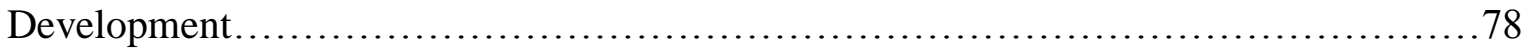

3.4 The Development of a Web-Based GIS Prototype............................83

3.5 Statistical Treatment of Stationary Spill Data..............................85 
3.5.1 Stationary Spill Sub-Database.................................. 85

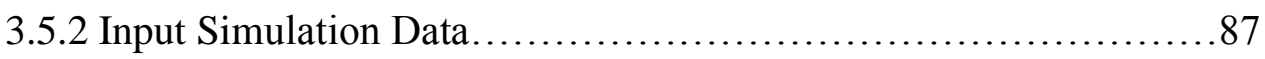

3.6 Macro Development \& Simulation Data.................................. 88

3.7 Spill Path \& Travel-Time Model Development.............................96

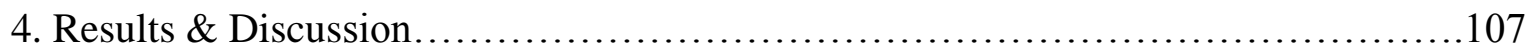

4.1 Analysis of Main Inland Oil Spill Database................................107

4.2 Spatial Analysis of Transportation-Related Spills \& Highway Spill Model...115

4.3 A Simple Prototype of a Web-Based GIS System for Inland Oil Spill...........

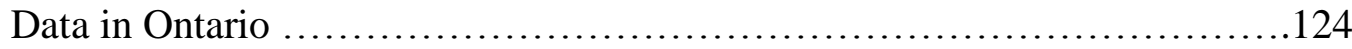

4.4 Statistical Analysis of Main Spill Database \& Stationary Spill Sub-Database.125

4.5 Simulation Data Analysis \& Macro Program Results.........................135

4.5.1 Input Simulation Data Analysis...................................135

4.5.2 Macro Program Development \& Output Simulation Data Results....144

4.6 Analysis of Spill Path \& Travel-Time Model Results.......................158

5. Application of Stationary and Transportation-Related Spill Models to the Spill Management

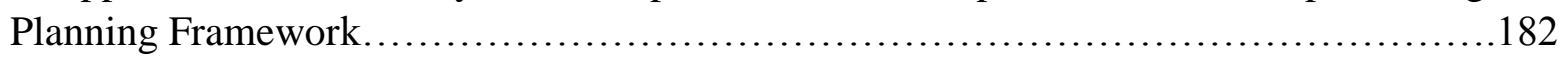

5.1 Development of Planning Tools for the Stationary Spill Model................182

5.1.1 Stationary Spill Model Planning Tools for Spill Prevention...........182

5.1.2 Stationary Spill Model Planning Tools for Spill Control................184

5.1.3 Stationary Spill Model Planning Tools for Spill Response \& Clean-Up.190

5.2 Development of Planning Tools for the Transportation-Related Spill Model...194

5.2.1 Transportation-Related Planning Tools for Spill Prevention...........194

5.2.2 Transportation-Related Planning Tools for Spill Control..............198

5.2.3 Transportation-Related Planning Tools for Spill Response \& Clean-Up.199

6. Conclusion. .202

APPENDIX A.1: Spill Path \& Travel-Time Model Development Displayed in three Main Parts. $.207-209$

APPENDIX B.1: Types of Environments Impacted by Inland Oil Spills in Ontario.......210 APPENDIX C.1: A Map Service of Transportation-Related Spills with Other Data........211 


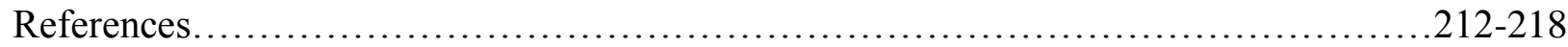




\section{List of Tables}

Table 1. Annual worldwide oil spillage in marine environments.....................6

Table 2. Previous research work on spill management carried out at Ryerson

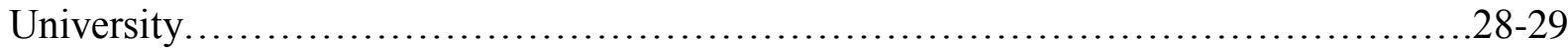

Table 3. Potential advantages and disadvantages of spills in land vs. water environments...36

Table 4. SAC Material Group Categories and Contaminant Codes.......................67-68

Table 5. Current vs. ideally recorded inland oil spill characteristics.....................111

Table 6. Descriptive statistics for the historical gasoline spill set of Humber River

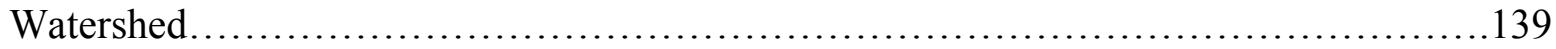

Table 7. Correlation matrix for numeric variables of the input simulation data set.......140

Table 8 . Text labels for cause variable categories................................. 150

Table 9. Descriptive statistics for three numeric variables of aggregate output simulation

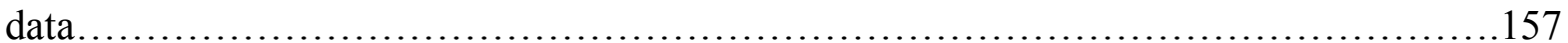




\section{List of Figures}

Figure 1. Histogram of values for percentage of Spill Volume (L) cleaned up as given in the $\%$ Clean-Up column of the spill database..........................................

Figure 2. Spill data records classified into categories based on the source of the spill.......24

Figure 3. The case study area for transportation-related spills which entailed the network of major highways in the City of Toronto.......................................... 25

Figure 4. The case study area for stationary spills which entailed the lower region of the Humber

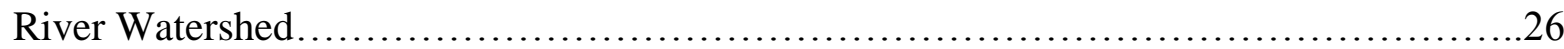

Figure 5. Structure of the probabilistic spill occurrence model..........................42

Figure 6. MATLAB-based Monte Carlo Simulation Model..............................43

Figure 7. Flow diagram of general overview of the methodology.......................65

Figure 8. A bar chart of the causes of all inland oil spills based on preliminary analyses...109

Figure 9. A bar chart of the reasons of all inland oil spills based on preliminary analyses...109

Figure 10. Type of inland oil spill as determined by the source of the spill.................114

Figure 11. Statistically significant hot and cold spots of inland transportation-related oil spills in

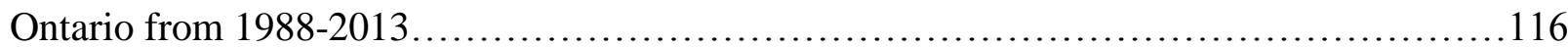

Figure 12. Optimized hot spot analysis using a hexagon polygon mesh for transportation-related inland oil spills in Ontario from 1988-2013 ...................................... 118

Figure 13 Spill points overlaid on the hexagon mesh of the optimized hotspot analysis

Figure 14. A map of a statistically significant hotspot beginning at the lakefront area in downtown Toronto

Figure 15. A map of spills within $50 \mathrm{~m}$ of major Toronto highways, often occurring at interchanges

Figure 16. Map service illustrating transportation-related land-based oil spills in Ontario....124

Figure 17. The retrieval of spill information by selecting spill points on the map service......125

Figure 18. An S-shaped pattern formed by the observed residuals........................127

Figure 19. Histogram of SAC oil type categories................................. 128

Figure 20. Scree plot of first PCA of initial stationary spill sub-database.....................130

Figure 21. The plot of factor coordinates for the first PCA experiment with all variables as active variables 
Figure 22. Loading scatterplot of component 1 and component $2 \ldots \ldots \ldots \ldots \ldots \ldots \ldots \ldots \ldots \ldots$

Figure 23. Groups of gasoline spill points with similar characteristics shown as colour-coded

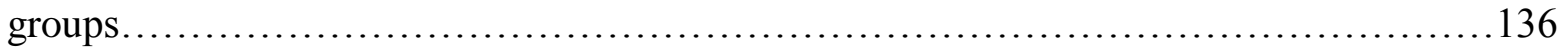

Figure 24. Lower region of Humber Watershed within the City of Toronto.................138

Figure 25. Bivariate histogram of categorical variables illustrating reason against cause.

Figure 26. Bivariate histogram illustrating spill cause versus spill sector.

Figure 27. Number of spills vs. number of simulations over a 10-year time period.....146

Figure 28. Number of spills vs. number of simulations over a 20-year time period.....146

Figure 29. Number of spills plotted against the number of simulations for the 10-year time period.

Figure 30. Illustration of mean interevent time (days) for each of 15,000 simulations...149

Figure 31. Histogram of mean of interevent time (days) for each random sample........151

Figure 32. Histogram of mean of spill mass $(\mathrm{kg})$ for each random sample.............152

Figure 33. Histogram of mean of \% clean-up for each random sample................152

Figure 34. Log-normal distribution overlaid on histogram of interevent time (days)....154

Figure 35. Histogram of $\%$ clean-up with a normal distribution fit type................155

Figure 36. Illustration of Toronto DEM after initial adjustments..................... 160

Figure 37. An example of an odd grouping of lines seen throughout the DEM both before and after the DEM was modified.............................................. 161

Figure 38. The slope raster resulting from the DEM input raster....................162

Figure 39. Some important GIS data layers used for model development............... 163

Figure 40. A close view of many GIS data layers used for model development...........164

Figure 41. An individual spill point and the direction coding legend for the underlying flow direction raster......................................................... 165

Figure 42. The output cost distance raster resulting from a cost distance analysis tool underlying the access points layer (blue points) for the study area

Figure 43. The output cost backlink raster resulting from a cost distance analysis tool.

Figure 44. Examples of least-cost paths of the output polyline feature class created.....172

Figure 45. The highlighted spill path contacts both points to connect them..... .173 
Figure 46. A short spill path does not connect the spill point (green) to the access point

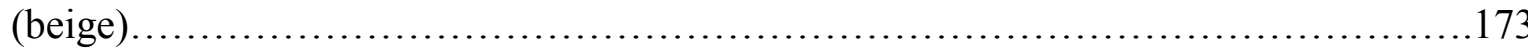

Figure 47. A typical line in the output polyline feature class that approximates the least-cost path between the spill point (green) and the access point (beige)...

Figure 48. A portion of the geometric network developed and used in the spill path and travel-time model............................................................. 176

Figure 49. Geometric network without additional junction end points................177

Figure 50. Three output files resulting from the tracing of spill pathways through the sewer

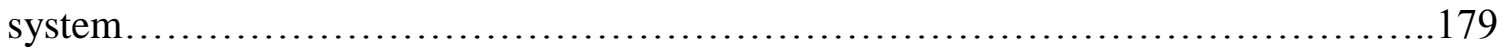

Figure 51. A section of an example of the final result of the spill path and travel-time

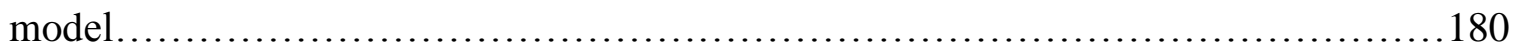

Figure 52. A typical design of a stormwater-collection system with gasoline spills......185

Figure 53. A sketch of an example of a catch basin insert to target oil and grease......187

Figure 54. A sketch of an oil-water separator used to trap free-phase oils and grease...188

Figure 55. Storage of spill clean-up supplies and equipment near a spill exit point.....194

Figure 56. A curve radius and sight distance at the entrance ramp of an interchange...196

Figure 57. A sketch of a cloverleaf interchange with spill clean-up kits placed inside each quadrant................................................................200 


\section{List of Appendices}

APPENDIX A.1: Spill Path \& Travel-Time Model Development Displayed in three Main

Parts.........................................................207-209

APPENDIX B.1: Types of Environments Impacted by Inland Oil Spills in Ontario.........210

APPENDIX C.1: A Map Service of Transportation-Related Spills with Other Data........211 


\section{Dissertation Structure}

This dissertation consists of six chapters and three appendices. Chapter 1 introduces the topic of oil spill management with respect to recent news issues and controversies. Background knowledge regarding oil and oil spills is then discussed, followed by a brief history of oil spills. Government programs and legislation in regards to spills and spill management is presented. The chapter concludes with a discussion of the objectives and scope of the research.

Chapter 2 is a literature review comprised of four sections which begins with a review of spill database analysis and spill data issues, the three-stage planning framework, and methods for modelling on-land oil spills. Chapter 3 first presents the overall methodology and then discusses the compilation of the main spill database and sub-databases. A flow chart of the overall methodology is shown in Figure 7. The methodology developed is comprised of four main components. The development of the highway spill model as it relates to transportation-related spills is discussed, followed by the statistical treatment of the stationary spill sub-database. The

development of the macro program and the associated simulations are then discussed. Lastly, the development of the spill path and travel-time model is presented.

Chapter 4 presents and discusses the results of each of the major elements of the methodology. The development of the main inland oil spill database is analyzed. The results from the spatial analyses of transportation-related spills are presented as part of the highway spill model. The statistical analysis of the main spill database and stationary spill sub-database is addressed. The simulation output results from the macro program are then analyzed. The results of the spill path and travel-time model are illustrated and analyzed. Chapter 5 links the results in Chapter 4 to each of the three stages of the spill management framework. Chapter 6 concludes the study with a review of significant research contributions and possible areas for improvement. 


\section{Chapter 1: Introduction}

Oil spill events usually bring to mind images of big ships situated by ocean shorelines full of wildlife covered in oil, however many major oil spill events occur on land and minor oil spill events occur in land environments every day. Oil spill events originating from sources on land, known as on-land or inland oil spills, may seem less alarming than major oil spill events in marine environment yet these events occur much more frequently. Inland oil spill events also have the potential for significant harmful impacts on local populations and environments by, for example, contaminating the water supply.

In this chapter, the terms oil, oil spills and oil discharges are first defined, followed by a discussion of relatively recent oil spill issues and controversies. Background knowledge pertaining to oil spills is then presented followed by a brief history of major oil spill events and increased awareness regarding spills. A description of government programs and legislation is then given, as well as the main problems addressed by the study and the objectives and scope of the research.

\subsection{Recent Oil Spill Issues and Controversies}

The term oil is a general term that is used to describe a wide variety of natural substances with varying origins such as plant, animal, or mineral, in addition to a wide range of synthetic compounds (Fingas et al., 2011). The terms oil spills and oil discharges are often used interchangeably yet differ in meaning. A spill is a discrete event in which oil is accidentally or, sometimes, intentionally released, often in an uncontrollable manner. A 
discharge is a legally permitted release of oil, usually in a highly diluted state in water, as part of normal operations (Fingas et al., 2011). Inland oil spills are also referred to as land spills as well as land-based or onshore spills.

Pipeline spills, transporting large quantities of crude oil and refined fuels, produce the highest volume of oil spilled on land. Most of the oil spills on land take place at petroleum production facilities, production collection facilities, battery sites, and wells (Fingas et al., 2011). Inland oil spills are the main reason for increasing controversies surrounding oil pipeline installations and developments yet the focus of public concern is often on marine spills resulting from the transport of oil to land environments.

The well-known Trans Mountain pipeline expansion in western Canada has been projected to generate billions in revenues but would result in a significant increase in the number of tankers carrying crude oil through the coastal waters of British Columbia, as well as the associated risk of spills (Weigh Anchor, 2018). When the federal government approved the expansion of the Trans Mountain pipeline, a promise was made to improve marine oil spill protection. The delay in the pipeline expansion in 2018 has been suggested to indicate that persistent gaps in oil spill response have yet to be rectified (Hunter, 2018). The issues surrounding oil pipeline spills, both locally and abroad, illustrate the problems and lack of preparedness with respect to the response and clean-up efforts of oil spills, for both land and marine environments.

For instance, the Trans Mountain pipeline expansion demonstrates some of the issues and concerns regarding oceanic tanker spills while ignoring many of the risks associated with 
inland oil spills from pipelines. A lack of emphasis on the central issues with respect to oil pipeline spills is also evident in other news articles which report on significant pipeline projects - even when the proposed pipelines are situated at some distance from marine environments. It is also common for spill response and clean-up measures in regards to inland oil spills from pipelines to be completely overlooked.

The largest inland oil spill in U.S. history occurred on July 25, 2010 yet did not receive any media attention at the national level (Hennighausen, 2012; McDiarmid, 2013). It occurred in Kalamazoo, Michigan, just 10 days after the well was finally capped in order to stop the largest oil spill in the history of the United States (U.S.), the Deepwater Horizon accident in the Gulf of Mexico. The Deepwater Horizon accident occurred in April through July 2010 and received a considerable amount of media attention.

A ruptured pipeline, belonging to a U.S. affiliate of Enbridge Inc., released more than 1.1 million gallons of heavy crude oil into the Kalamazoo River in Michigan. The rupture was caused by a 6.5 foot tear in a 30 -inch carbon steel pipeline. According to the National Transportation Safety Board, the company was aware of the cracks and corrosion in the pipeline for at least five years before the spill (Hennighausen, 2012). The spill event is the most expensive pipeline accident in the U.S. and has cost the company over one billion dollars (McDiarmid, 2013).

The pipeline accident in Michigan has raised alarm in regards to safety and spill response and disaster preparedness by the City of Toronto and surrounding municipalities, when Enbridge attempted to expand a pipeline carrying oil from east to west through critical 
urban areas. The pipeline currently runs beneath Finch subway station at a depth of less than two metres below the sidewalk and a little more than half of a metre above the subway structure.

According to the City of Toronto, there was no contingency plan in place by all relevant authorities if a petroleum leak were to occur near the entrances of the subway station (McDiarmid, 2013). Municipal fire departments would act as first responders in the event of a spill since Enbridge's response time of between 90 minutes and 4 hours is viewed as too slow by the implicated municipalities. Enbridge's emergency manual has been criticized by Toronto Fire Services as being "generic" as it lacks a response specific to Toronto. This example demonstrates the need for more specific clean-up and response measures for managing urban inland oil spills.

In 2018, a similar project was proposed by Imperial Oil known as the Imperial Oil Waterdown to Finch Project. The project aims to replace an aging 63-kilometre section of pipeline between a rural Waterdown pumping station in Hamilton and a storage facility in Toronto (Van Dongen, 2018). The pipeline carries diesel and aviation fuel from Sarnia to Toronto through Waterdown and Burlington. Discussions were held regarding the potential impact of the project on environmentally sensitive lands, endangered species and waterways in or near Hamilton (Van Dongen, 2018).

Although the environmental impacts of the project were being considered, the risk of a spill or spill response was not mentioned. Some concern was raised in regards to the ongoing flow of oil in relation to alternative forms of energy (Van Dongen, 2018). 
According to peak oil theory, global oil consumption is expected to peak in the late 2030s with an accompanying increase in renewable energy sources. Examples of renewable energy sources include wind power, solar power, biomass, geothermal energy, and hydroelectric power (Cooper \& Johnson, 2018). Aviation fuel or jet fuel may not be as easily replaced as other types of oils because planes require a powerful burst of energy to lift off. A pipeline carrying jet fuel may therefore be required for some time (Cooper \& Johnson, 2018).

Oil spill catastrophes have become relatively rare in recent years due to the implementation and enforcement of prevention measures as well as more responsible operations by shipping and oil industries. This has resulted in a global downward trend in oil spills over the past several decades. Global oil spillage rates have decreased significantly since the 1960 s and 1970 s from approximately 635,000 tons per year to approximately 300,000 tons per year from all sources, excluding the intentional spillage during the 1991 Gulf War. Sporadic increases in annual spill amounts can be attributed to one or two especially large oil spill events occurring in a particular year (Fingas et al., 2011). The reduction in marine oil spill disasters or catastrophes due to prevention measures as well as co-operation by certain industries suggests that if more prevention measures are applied to land environments, a reduction in on-land oil spills can be achieved.

From approximately 1990 to 2010, the largest sources of oil spills in marine or oceanic environments have been related to oil transportation by tank ships (tankers) or through 
pipelines. Oil spills from pipelines therefore also contribute significantly to spilled oil in marine environments. Various sources which contribute to oil spills in marine environments are listed in Table 1.

Table 1. Annual worldwide oil spillage in marine environments.

\begin{tabular}{|l|l|l|l|}
\hline & \multicolumn{3}{|c|}{ Estimated Average Annual Tons Spilled (excluding } \\
war-related spillage)
\end{tabular}

(Source: Adapted from Fingas et al., 2011)

Table 1 demonstrates a significant reduction in the total volume of oil spills in marine environments from the 1970s to the 1990s. This can be regarded as a remarkable accomplishment considering the increases in production, shipping, and handling of oil that occurred during this time (Fingas et al., 2011). The progress demonstrated by the reduction in marine spills indicates potential improvement for reducing oil spills in land environments as well.

"Urban runoff", with respect to oil spill analysis, is composed of accumulated drops of oil that leak from trucks, automobiles, and other vehicles in addition to small, chronic spillages that result from other land-based sources. The accumulated oil washes off into culverts, storm sewers, and other water bodies. The oil that washes off into water bodies such as streams and rivers may reach the coastal or marine environment and a substantial 
quantity could be involved. Annual oil inputs from urban runoff into U.S. marine waters approximate 50,000 tons which is approximately one-sixth of the annual worldwide oil spillage in marine environments during the 1990s. Since the exact source of this type of oil spillage is often difficult to determine, it is classified as nonpoint source pollution (Fingas et al., 2011).

It is a common misconception among the public that oil spills from tankers are the main source of oil pollution in the marine environment. Only $5 \%$ of all oil pollution on the seas is from tankers. Half of the oil spilled in the seas is from runoff of oil and fuel from land-based sources rather than from accidental spills on water (Fingas et al., 2011). Oil spills in land and freshwater environments can therefore have a significant impact on marine environments.

Oil spills that originate in marine environments, on the other hand, can also permeate into freshwater and land environments. Inland oil spills first impact local environments rather than marine environments since oil spills that originate on land flow downslope like water, except in rare circumstances, and often collect in water bodies such as rivers, creeks, ditches, and streams (Owens, 2002). Since oil spills can originate from and spread to various environments, it is important to understand how wildlife and vegetation inhabiting different environments react to oil spill events (Saba and Spotila, 2003).

Wildlife and vegetation in land environments can even be negatively impacted from small, inland oil spills. In Ontario, small industrial oil spills occur on a weekly basis. Each spill has the capacity to affect hundreds of animals and cause significant damages 
on sensitive wildlife habitat. Although clean-up companies are usually the first to arrive at the site, they often lack the training required to treat animals affected by such accidents. The Toronto Wildlife Centre is one of the only wildlife centres in Ontario, Canada, with staff capable of responding to oil-exposed animals (The Toronto Wildlife Centre, 2013).

Well-publicized oil spill disasters are usually much larger in scope than typical oil spill events and account for a large portion of the overall total volume of oil spilled (Han, 2007; Fingas et al., 2011). Oil spills with more than 30 tons spilled are the largest spill events which account for only $0.1 \%$ of all oil spill events yet comprise nearly $60 \%$ of the total volume spilled. Common, smaller oil spill events release 0.003 to 0.03 tons of oil and account for $72 \%$ of all spills yet comprise only $0.4 \%$ of total spillage volume (Fingas et al., 2011).

The majority of oil spill events are relatively small and occur daily by the hundreds or even thousands on a global scale. These oil spills can be considered as "routine" events that can be easily responded to using local resources (Fingas et al., 2011). In spite of the general downward trend in oil spill events, they continue to pose a persistent threat to environments and communities throughout the world, which is why maintaining spill response preparedness is imperative (Kai, 2005; Fingas et al., 2011).

Although large oil spill events occur infrequently, they attract the most attention from the public and the media because they are more visible and exert a greater impact in some ways. For any event, the size of a spill cannot be used to directly measure the damage. 
Location and oil type are also very important factors in assessing the extent of environmental and socioeconomic damage. The attention drawn by major oil spills in recent years has raised awareness of the risks and associated environmental damage of oil spills; however they are not yet recognized by the public as a daily fact of life (Fingas et al., 2011).

In Canada, the majority of oil spills occur on land and constitute a large portion of the total volume spilled (Han, 2007). Approximately $80 \%$ of the total annual volume (tons) of oil spilled are land spills while the remaining $20 \%$ are marine spills. Similarly, approximately $90 \%$ of the number of annual oil spills are inland oil spills while the remaining $10 \%$ are marine oil spills (Han, 2007). Spills categorized as marine represent the proportion of all annual spills in Canada that enter directly into the ocean or navigable waters (Han, 2007).

\subsection{Background}

Oils are mainly composed of hydrocarbon compounds, compounds made of hydrogen and carbon (Fingas et al., 2011). Although the primary elements in oil are hydrogen and carbon, oils also contain varying amounts of sulphur, nitrogen, oxygen, and the occasional mineral salts. Trace metals such as nickel, vanadium, and chromium can also be found in oil. Mixtures of hydrocarbon compounds form crude oils which can range from smaller, volatile compounds to very large, non-volatile compounds. Crude oils are commonly known as the raw products of oil and are used in the oil production process to create petroleum products for transport, heating, and chemical synthesis. There are many 
different kinds of crude oils with varying compositions which contain many compounds of different sizes and different classes, and new compounds are continuously being discovered (Fingas et al., 2011).

The mixture of hydrocarbon compounds in crude oil depends on the geological formation of the area in which the oil is found. This mixture exerts a strong influence on the properties of the oil, giving each oil or petroleum product certain unique characteristics or properties that influence how the oil behaves when spilled. These characteristics or properties determine the fate and effects of the oil in the environment and influence the efficiency of clean-up operations. Spill events entail many different kinds of oil that range from varieties of crude oil to a large assortment of refined products that may even include cooking oil. The spilled oil may also range from heavy persistent fuels to lighter, less persistent, but very toxic lighter fuels (Fingas et al., 2011).

Oil spill events occur under many different conditions and circumstances such as oil type and volume, season, weather effects, distance to sensitive resources, and currents. Consequently, each oil spill event is relatively unique as well as the resulting impact, damages, and response challenges. Regional and national patterns of spillage are determined by the type of oil-related activities in areas where oil spills occur, the volume of oil handled, and the extent to which oil prevention measures have been implemented and enforced (Fingas et al., 2011). 
Oil spills are considered as a form of pollution and can occur anywhere that oil is produced, transported, stored, or consumed which includes land, ocean, and inland freshwater systems.

In general, a spill of a pollutant is defined by the Environmental Protection Act as: "... a discharge into the natural environment, from or out of a structure, vehicle or other container, and that is abnormal in quantity or quality in light of all the circumstances of the discharge." (Part X, Section 91(1)).

A "wet spill" occurs during rainfall and a "dry spill" occurs during dry weather conditions (Spills Action Centre [SAC], 1993).

Oil spills and discharges can occur at any point in the "life cycle" of oil in which raw oil products such as crude oil are transformed into refined oil products such as petroleum. An accident can occur at each step or storage period during the life cycle of oil within the oil industry. This includes the oil exploration and production stages, as well as transport by vessel, pipeline, railroad, or tanker truck. Other stages are refinement, storage, waste disposal, or consumption or usage of oil as a fuel or as a raw material for manufacturing (Fingas et al., 2011).

From the oil fields to the consumer, the movement of oil entails as many as 10 to 15 transfers between various modes of transportation. While oil is being transported, it is stored at transfer points and at terminals and refineries (Fingas et al., 2011). An oil spill can potentially occur whenever oil is transferred during exploration and production activities and while in transport by tank ships, pipelines, and railroad tank cars to the refineries. In the refineries, the oil is refined to create the various types of fuels that are 
then transported by pipeline, rail, truck or tank vessels to consumers, providing more opportunities for oil spill events to occur (Fingas et al., 2011).

Consumption-related spillage stems from manufacturing facilities, non-tank vessels (cargo ships) that carry oil only as fuel and for machinery, tanker trucks bringing oils to service stations and heating oil tanks, as well as many miscellaneous sources. Structural failures, weather-related events, operational errors, earthquakes, vandalism, political conflicts and human errors and negligence are all possible reasons why spills occur. According to spill experts, an estimated $30 \%$ to $50 \%$ of oil spills are either directly or indirectly caused by human error and $20 \%$ to $40 \%$ of these incidents are caused by equipment failure or malfunction (Fingas et al., 2011).

Inland oil spills and other chemical land spills are considered to be one of the major contributors to pollution in the Greater Toronto Area (GTA) and the Great Lakes Region (Li, 2005; Cao, 2013). The detrimental effects of inland oil spills extend to air, water, and soil environments ( $\mathrm{Li}, 2005)$. Possible negative impacts to these environments include impairment of drinking water quality, destruction of freshwater invertebrates and vertebrates, and contamination of surface water and groundwater (Cao, 2013).

Urban environments can be negatively impacted by large quantities of inland oil spills in many ways. The toxicity of the spilled oil that runs off of impervious surfaces such as concrete and seeps into creeks can poison exposed organisms and destroy their natural habitats through processes such as algal bloom. Sewage treatment is more difficult as a result of spilled oil entering sanitary sewers. If spilled oil enters storm sewers and 
becomes ignited, a major fire hazard exists because of the potential for fire and smoke to spread throughout the whole neighbourhood (Chui, 2002). Land-based oil spills in urban areas can therefore endanger public health, cause economic disturbances, and damage natural resources (Han, 2007).

\section{$1.3 \underline{\text { A Brief History of Oil Spills }}$}

Oil spills do not always occur because of anthropogenic or human-related activities. Natural leakages of oil from the Earth have been occurring since prehistoric times, as evidenced by commonly visible oil slicks on water and oiled shorelines. Liquid and gaseous hydrocarbons leak out of the ground from natural springs known as "natural seeps" that release a substantial amount of crude oil annually. Oil seeps are supplied by natural underground accumulations of oil and natural gas. As a result of geological processes, oil from submarine and inland subterranean oil reservoirs rise to the surface each year. Natural seeps often release oil in small amounts at regular intervals but sometimes release oil in larger amounts, having the same impact on the environment as crude oil spills from tankers or other sources (Fingas et al., 2011).

Historical recordings of natural discharges of petroleum seeps can be found in the writings of Herodotus and Marco Polo (Fingas et al., 2011). It has also been shown that Native American groups living in California used products made from oil seeps long before European settlement. Although the results have not been openly published in scientific or technical literature, locations of natural seeps have been used in recent times to determine potential locations for oil exploration and production (Fingas et al., 2011). 
An enhanced public awareness of general environmental issues emerged during the late 1960s. The combination of greater public awareness and several larger anthropogenic oil spills that occurred at this time brought the concern over oil pollution to the forefront. Before this period, many oil spills occurred during wartime, such as the many oil tank ships that were torpedoed and sunk during World War II. A total of 484,200 tons of oil were released from torpedoed tankers within 90 kilometers of the United States coast, amounting to approximately 20,000 tons of oil per week over the first six months of 1942 (Fingas et al., 2011). Oil spills resulting from war efforts did not raise much concern, however, as they were associated with wartime. There was also a general lack of awareness of environmental protection during these times (Fingas et al., 2011).

Over the last several decades, sunken war ships have been a source of environmental concern because they release oil in the form of leakages and discharges, with the potential to continue in the future. In a study by Michel et al. (2005), most sunken vessels in the open ocean were found to have begun to release small amounts of oil over a relatively long period of time or had the potential to do so, as opposed to releasing a large quantity all at once. By releasing small quantities over time, a vessel behaves similarly to a "natural seep" (Michel et al., 2005; Fingas et al., 2011).

The location of the shipwreck plays a significant role in determining the potential of the impact since those closer to the shoreline are more threatening for land environments. In 2005, a database was developed that recorded shipwrecks that met a certain minimum 
weight and carried oil. The estimated amount of oil contained in shipwrecks throughout the world is 2.5 to 20.4 million tons (Michel et al., 2005; Fingas et al., 2011).

The first noteworthy major oil spill, the Torrey Canyon, occurred in 1967. Although it was not the first oil tanker spill, it was the largest spill to date and incited a large public outcry. Highly toxic first-generation dispersant chemicals (chemical spill-treating agents used to clean up or remove oil) were used to treat the spill but only exacerbated the environmental damage. Other significant oil spills occurred throughout the world during the 1970s, bringing greater attention to the issue on an international level. In 1979, the largest tanker spill of all time, the Atlantic Empress, occurred in Trinidad and Tobago/Barbados while the largest non-war-related spill in history occurred in the Gulf of Mexico, the Ixtoc I well blowout (Fingas et al., 2011).

Perhaps the most notorious, though not the largest, oil spill event is the 1989 tanker spill in Alaska known as Exxon Valdez, which spilled over 37,000 tons of crude oil onto what was considered to be a pristine location. The spillage resulted in the most costly and lengthy spill response and damage settlements in history and the ramifications were felt all over the globe. Resultantly, the Oil Pollution Act (OPA) of 1990 was enacted in the United States which included significant spill prevention and liability legislation. International conventions on spill prevention included measures such as the requirement for double-hulls (a type of design for the lower, waterproof body of a ship) on tankers by 2015 and increased financial liability (Fingas et al., 2011). 


\subsection{Government Programs and Legislation}

Government and industry are both striving to lessen oil spill risk by introducing strict new legislation and stringent operating codes. The prevention of oil spills minimizes the number of oil spills, leading to a more highly protected environment. New operating and maintenance procedures have been put in place by industry to reduce accidents leading to spills (Fingas et al., 2011).

Government fines, high clean-up costs and reputational loss or damage also act as deterrents to oil spills. Using an assumed currency of U.S. dollars, clean-up costs can be compared between countries. For example, it costs an average of $\$ 20$ to clean up each litre of oil spilled in Canada and an average of approximately $\$ 100$ per litre spilled in the United States (Fingas et al., 2011). Globally, the average cost of clean-up ranges from \$20 to \$200 per litre depending on oil type and location of spillage (Fingas et al., 2011). The most expensive kind of clean-up process is usually the cleaning up of oil on shorelines (Fingas et al., 2011). The higher average cost per litre in the U.S. relative to the average cost per litre in Canada indicates that there may be higher incidences of oil spills on U.S. shorelines or other location types that are more difficult to clean-up. There may also be more spills of oil types that present greater challenges for spill clean-up.

Methods for controlling and cleaning up oil spills must also be implemented and devised. The different sizes and impacts of oil spills require different response levels. It has been suggested that an integrated system of contingency plans and response options be used to facilitate and ameliorate oil spill responses, leading to a reduction in the environmental 
impact and severity of a spill. The aim of a contingency plan is to coordinate all treatment options of the response to an oil spill. A contingency plan could involve a control phase which includes stopping the flow of oil and containing the oil, followed by a clean-up phase. The unpredictable nature of environmental emergencies such as oil spills necessitates an effective response approach that must include preparations for unexpected situations and plans for spill countermeasures that can be executed even during worst-case scenarios (Fingas et al., 2011).

The coastal and inland waters of Canada are the common responsibility of federal, provincial, and territorial governments as well as several federal departments ("Acts", 2013). The management of spilled materials in Canada, including oil, is based on a legislative framework encompassing all levels of government as well as the individual responsibility of the discharger of the spill. Relevant regulations to spill management are contained in Federal Acts including the Canadian Environmental Protection Act, the Canada Shipping Act, and the Emergency Plans Act. Provincial Acts include the Ontario Water Resources Act and the Environmental Protection Act (Ontario), as well as municipal by-laws. Regulations within these acts pertain to prevention planning, notification to proper authorities, and clean-up and restoration of the natural environment (Li, 2002).

The Department of Environment Canada is the federal body of government responsible for managing over a dozen Acts of Parliament, either wholly or partly, by providing programs to administer these acts. The Department carries the responsibility for meeting 
several obligations delineated in the legislation under these acts. Two important pieces of water legislation administered by Environment Canada as part of environmental protection are the International Rivers Improvement Act (IRIA) and the Canada Water Act (“Acts”, 2013).

Environment Canada places a strong emphasis on pollution prevention (P2) to minimize or avoid creating pollutants and wastes. The Department believes that a proactive approach such as pollution prevention is potentially more effective when protecting the environment than treating or cleaning pollutants and waste. The focus of P2 is on the avoidance of pollutant creation instead of on the management of pollutants after they have been created ("Pollution Prevention", 2013). Four acts administered by Environment Canada that fall under P2 are listed as follows:

\section{1) Canadian Environmental Protection Act 1999 (CEPA 1999) \\ 2) Fisheries Act \\ 3) Antarctic Environmental Protection Act (AEPA) \\ 4) Arctic Waters Pollution Prevention Act}

According to Environment Canada, P2 should not exist by itself but rather be part of an ongoing pollution management approach encompassing prevention, control, and clean-up (“Pollution Prevention”, 2013). The Department administers a pollution planning (P2) process in which organizations investigate present operations and devise a plan for decreasing or eliminating pollution at the source ("Pollution Prevention Planning", 2013).

The Federal Government of Canada also administers an Environmental Emergencies Program to reduce the frequency and occurrence of environmental emergencies concerning the accidental, unplanned or uncontrolled release of hazardous materials such 
as chemicals or oils. The Canadian environmental notification system uses federal-provincial/territorial 24-hour authorities as the first point of contact (“Environmental Emergencies", 2013).

The first point of contact for reporting the accidental, unplanned or uncontrolled release of hazardous materials such as chemicals or oils in Ontario is the Spills Action Centre (SAC) which is administered by the Ministry of the Environment, Conservation and Parks (formerly known as the Ontario Ministry of the Environment or MOE). SAC operates a 24-hour-7-day call centre that can be reached through a province-wide toll-free number. The range of notifications received by SAC is quite broad and can include reports of spills and other environmental discharges, adverse drinking water results, and disruptions to time-critical ministry services (“Spills Action Centre”, 2012).

SAC emerged from the Great Lakes Water Quality Agreement (GLWQA), first signed in 1972 by Canada and the United States. The agreement requires both countries to maintain the chemical, physical, and biological integrity of the Great Lakes basin ecosystem (Schardt, 2010). SAC began operations in 1985 on the same day that Part X of the Environmental Protection Act came into effect (Ontario Ministry of the Environment [MOE], 1994). The legislative authorities which govern SAC are the Environmental Protection Act (RSO 1990, Part X), the Ontario Water Resources Act (RSO 1990), and the Pesticides Act (RSO 1990). 
In accordance with the Emergency Management and Civil Protection Act (EMCP Act) and Regulation 380/04, Ontario municipalities and provincial ministries are required to have Emergency Management Programs. The five pillars of emergency management are:

1) prevention,

2) mitigation,

3) preparedness,

4) response, and

5) recovery.

These five pillars should support a comprehensive emergency management program which incorporates a risk-management approach ("Emergency Planning”, 2012).

A common method of promoting pollution prevention at the municipal level is through the implementation of sewer use by-laws. Sewer use by-laws can regulate activities that prevent spilled substances from entering sewers $(\mathrm{Li}, 2002)$. By embedding mandatory pollution prevention requirements into its sewer use by-law, the City of Toronto is trying to motivate businesses to find ways of avoiding, reducing or eliminating the creation of pollutants at the source (Han, 2007).

\section{5 $\underline{\text { Problem }}$}

The two main problems associated with inland oil spills are: (1) the frequency of inland oil spill events and (2) the impact of inland oil spill events. Although most oil spill events are small and occur throughout the world on a daily basis, inland oil spills usually occur in industrialized areas near urban centres and can therefore contaminate the 
drinking water supply of local populations (Fingas et al., 2011). Oils are also flammable substances that can pose threats to safety when spilled.

Preliminary analyses of the spill database developed in the present study has revealed a general lack of spill response and clean-up efforts. Most entries under the \% Clean-Up column, indicating the percentage of Spill Volume (L) cleaned up, are either blank or zero. Some of these entries were assumed to represent mistakes or unknown values. The composition of non-blank \% clean-up entries is shown in the histogram of Figure 1.

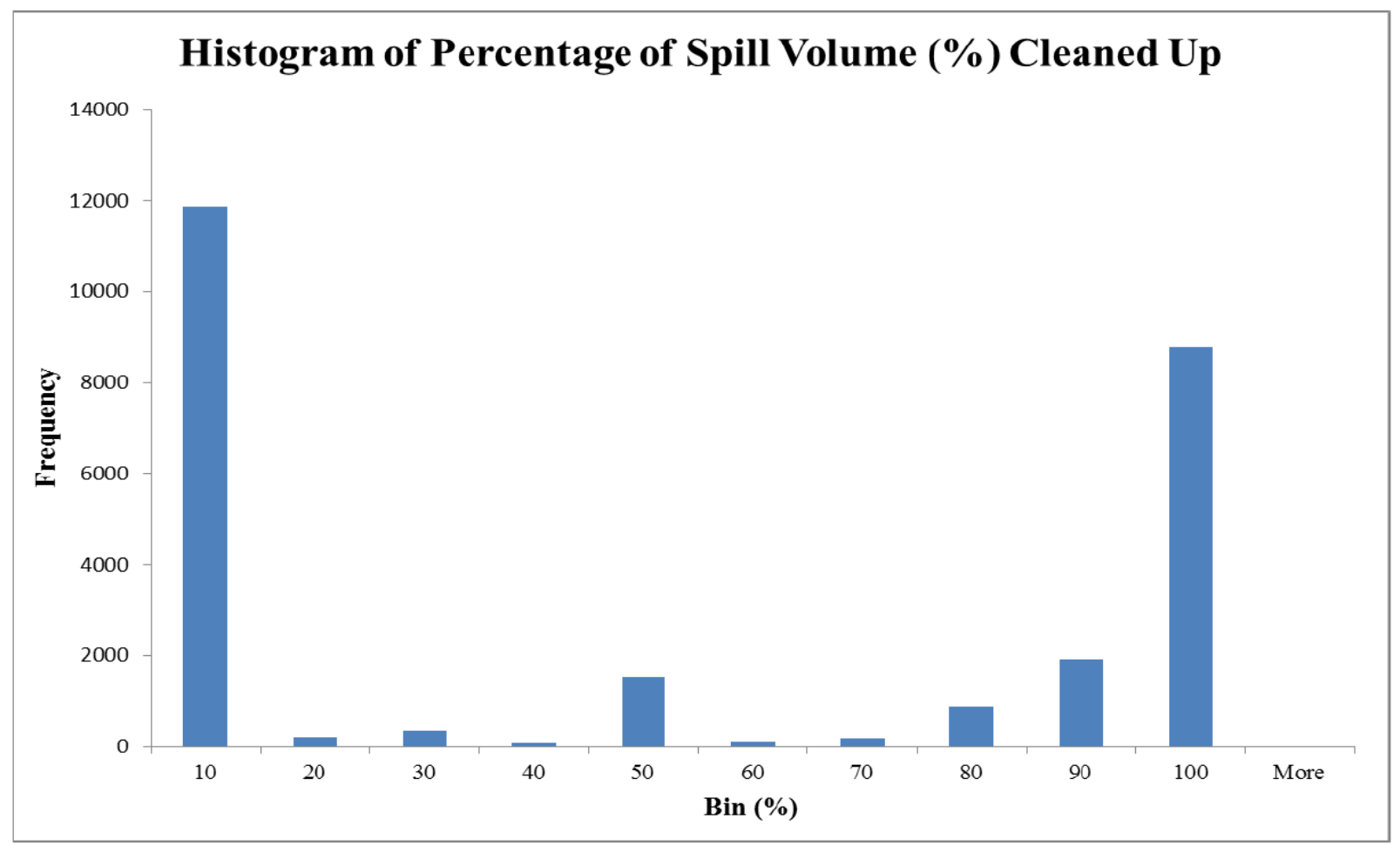

Figure 1. Histogram of values for percentage of Spill Volume (L) cleaned up as given in the $\%$ Clean-Up column of the spill database.

The histogram in Figure 1 illustrates how the Clean-Up (\%) figures for on-land oil spills in Ontario are either relatively low, indicating very little clean-up, or very high, indicating that most of the spill was cleaned up. A small portion of Clean-Up (\%) values 
fall in the middle or in between various intervals. The histogram of \% Clean-Up provides evidence to assert that there is a fairly significant problem with respect to spill response and clean-up strategies in Ontario.

The conventional focus by the public, media, and major environmental organizations has been on major oil spill events in marine environments. The majority of research efforts have therefore been directed towards marine oil spill events, leaving a tremendous gap in research with respect to inland oil spill events (Fingas et al., 2011). At present, there is no comprehensive spill management framework for urban municipalities, although many local municipalities have integrated some aspects of spill management into their procedures and policies.

\section{$1.6 \underline{\text { Objectives \& Scope }}$}

The overall objective of this study was to develop a comprehensive planning framework for urban inland oil spill management entailing models or tools for each of three main stages of spill management: (1) prevention, (2) control, and (3) response and clean-up. Oil spills occurring on land or originating from sources on land, inland oil spills, were the only type of oil spills considered. The framework involves both temporal and spatial aspects. The following situations or types of inland oil spill events were excluded from consideration:

- Real-time emergency responses or extreme events such as fires or disasters

- Spills at major hydro stations 
- Spills related to aircraft, trains, or watercraft (unless at marine terminals)

- Out-of-province spills

Out-of-province spills are inland oil spills originating in other provinces or in the U.S. Since the spill data provided was for Ontario only, out-of-province spills were considered as errors and removed from the database. Other kinds of spill records that were excluded, such as spills occurring at major hydro stations or as a result of being transported by train, involve different vehicles and equipment than the spills which were the focus of the study. The parameters and factors pertaining to these situations differ sufficiently enough from the rest of the spill data to have justified the exclusion of these kinds of spills from being studied.

Only spills impacting surface water environments with freshwater were included since they are within close proximity to the areas of study. Water bodies such as rivers and streams are considered to be surface water environments, as well as stormwater-collection systems that transport on-land oil spills to surface water environments. Therefore, inland oil spills which spilled directly into water bodies or were transported to such environments by stormwater-collection systems were retained. Oil spills impacting soil, air or other media without impacting surface water environments were excluded.

Although spill records entailing surface water impacts were included, the focus of the research was on the management and flow characteristics of spills on land. The 
management and flow characteristics of spills in receiving waters was not part of the study. Spill modelling was conducted for the on-land portion of the spill travel-path which begins at the point of spill occurrence, continues to the point of entry into the stormwater-collection system and ends at the exit point of the system. The travel-path after the spill enters the receiving water body was not addressed by this study.

The inland spill data was sub-divided into the following two categories based on the source of the spill event: (1) transportation-related spills and (2) stationary spills. If an inland oil spill occurred as a result of a moving vehicle or while being transported by a moving vehicle, it was categorized as transportation-related. If the spill occurred at a fixed point or location such as at a factory or at a gas station, it was categorized as stationary. Figure 2 illustrates the division of inland oil spill data records according to each spill category.

\section{Inland Spill Source Percentage}

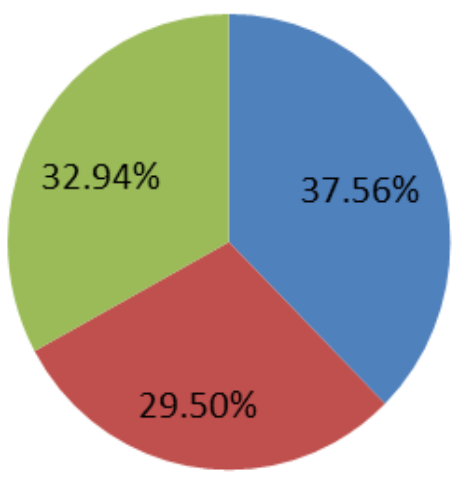

- Other/Unknown/Not

Applicable/Miscellaneous/Blank

- Stationary

- Transportation-Related

Figure 2. Spill data records classified into categories based on the source of the spill. 
The purpose of subdividing the spill data was to develop tools or models specifically for stationary and transportation-related spills in each of the three stages of the spill management framework. The case study area for transportation-related spills was the network of major highways within the City of Toronto as shown in Figure 3.

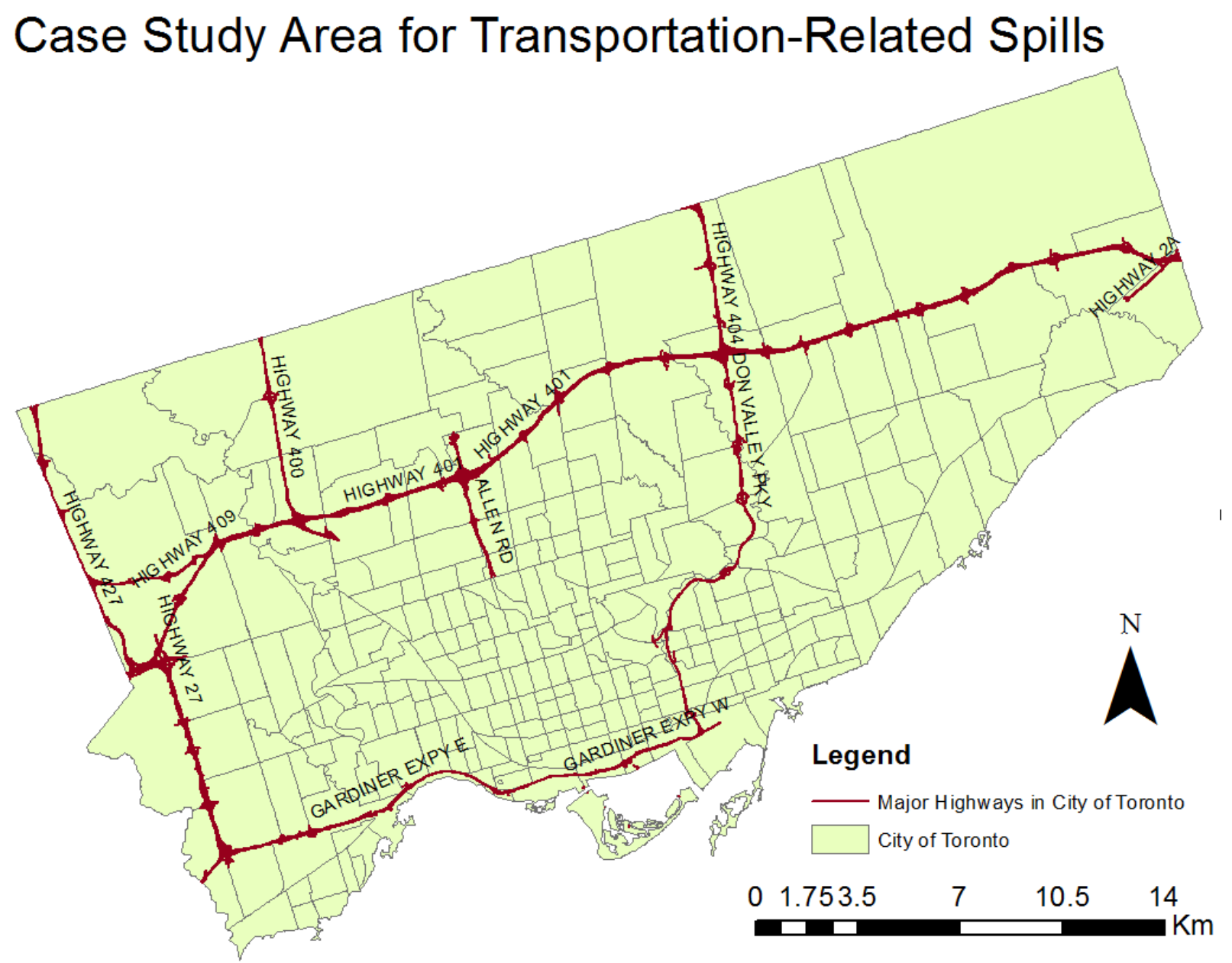

Figure 3. The case study area for transportation-related spills which entailed the network of major highways in the City of Toronto.

The case study area for stationary spills is the lower region of the Humber River

Watershed that encompasses the City of Toronto as shown next in Figure 4. 


\section{Case Study Area for Stationary Spills}

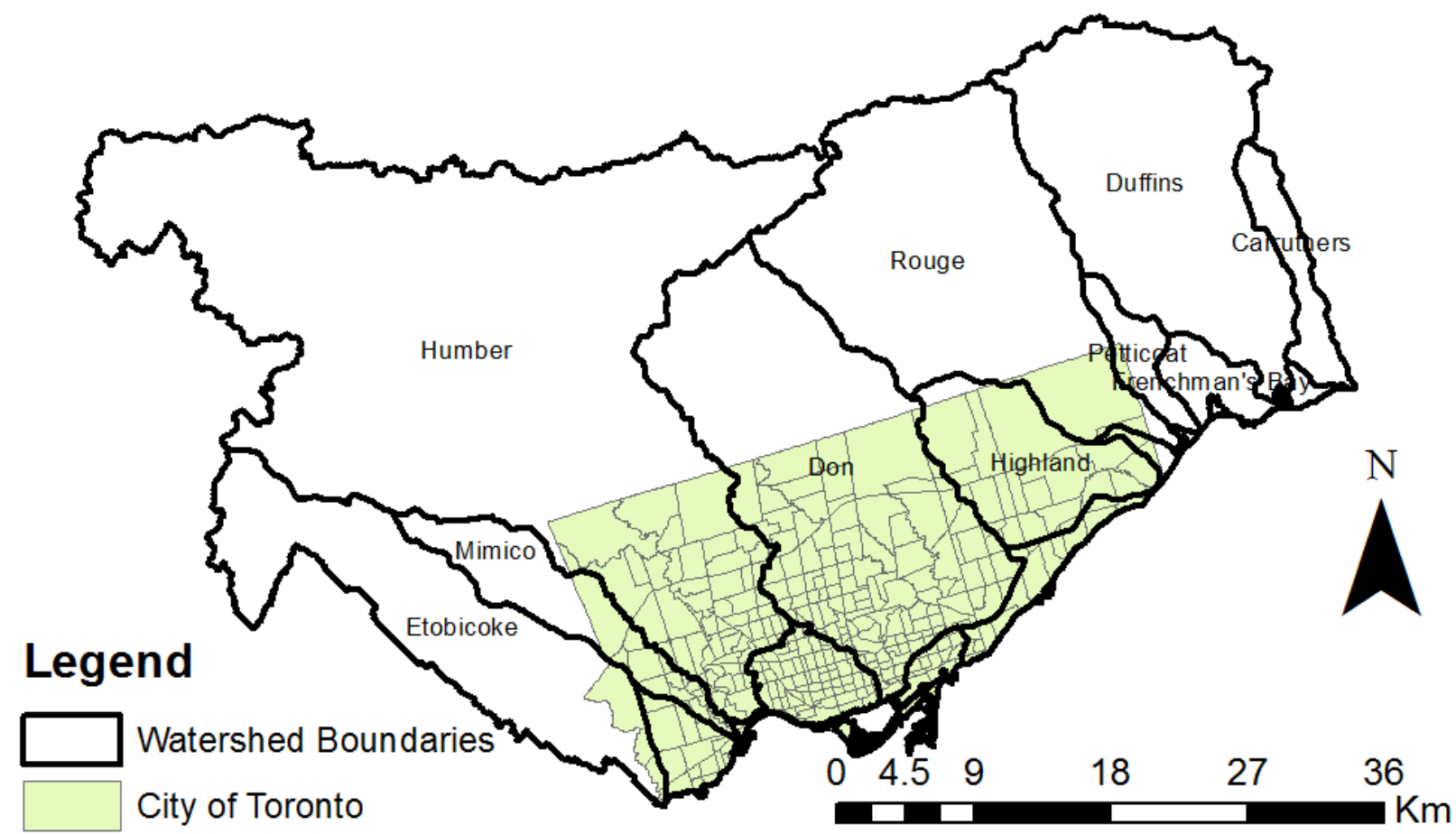

Figure 4. The case study area for stationary spills which entailed the lower region of the Humber River Watershed.

The Humber River Watershed is a relatively large watershed containing ten

municipalities. The lower region of the Humber Watershed is located in the west end of the City of Toronto. The entire watershed occupies an area of $911 \mathrm{~km}^{2}$ and is home to 856,200 people. Although it is heavily industrialized in some areas, the overall land use is still primarily rural with $32 \%$ natural cover (TRCA, 2014). 


\section{Chapter 2: Literature Review}

The inland spill data was compiled into a database to form a spill database which is an organized collection of spill data that is stored and accessed by a computer system. Since spill databases are the key component to developing spill management plans, other spill databases are reviewed in this chapter as well as previous research on inland oil spills. Issues pertaining to oil spill data are then discussed. Since the scope of the study entails inland oil spills which impact freshwater environments, a comparison between land spills and water spills is presented as well as a review of the impacts of oil spills on freshwater environments. Areas of increasing importance for spill management, such as the application of the three-stage framework (prevention, control and response), Web-based GIS and spill modelling software are then discussed.

\subsection{Spill Database Analysis}

Oil spills are usually recorded by the same authorities responsible for initiating emergency spill response operations. Environment Canada delegates this authority to localized provincial agencies such as the Spills Action Centre (SAC) in Ontario and collects spill information through the National Pollution Release Inventory (Tang, 2005; Environment and Climate Change Canada, 2013). Examples of American authorities include Coast Guard agencies or state and local governments (Fingas et al., 2011). As a result of the different governing bodies and polluting organizations compiling spill statistics, there can be considerable variation with respect to the accuracy of reporting, especially in regards to smaller spills (Tang, 2005; Fingas et al., 2011). 
A great deal of research on inland oil spill management has been conducted at Ryerson University in Ontario which is in the unique position of receiving comprehensive spill databases from SAC. During earlier work on spill management at Ryerson University, inland spill data containing spill records for various chemical and oil inland spills were obtained from SAC for the years of 1988 to 2006 inclusive.

A Microsoft Access spill database was developed at Ryerson University to store records for inland chemical and oil spills from 1988 to 2002 in separate tables. The database first developed for oil spill data from 1988 to 2002 and the data received from SAC for years 2003 to 2013 formed the repository of raw data for the development of all spill databases and sub-databases in the current study. The majority of spill characteristics from the data sets of both earlier (1988-2002) and later (2003-2013) time periods are often the same or similar (e.g. Oil Type, Spill Volume, Incident Date and/or Date Reported). Table 2 lists previous research work on spill management conducted at Ryerson University.

Table 2. Previous research work on spill management conducted at Ryerson University.

\begin{tabular}{|l|l|l|l|}
\hline Year & $\begin{array}{l}\text { Author (i.e. Name } \\
\text { of Student) }\end{array}$ & Title of Thesis or Work & Focus of Research \\
\hline 2002 & Chui, J. K. L. & $\begin{array}{l}\text { Control of Oil Spills in Urban } \\
\text { Areas }\end{array}$ & $\begin{array}{l}\text { *Design and physical } \\
\text { model study of oil spill } \\
\text { control device } \\
\text { *Spatial analysis }\end{array}$ \\
\hline 2004 & Fan, C. & $\begin{array}{l}\text { Hydraulic Analysis of Oil Spill } \\
\text { Control Systems in } \\
\text { Transformer Stations }\end{array}$ & $\begin{array}{l}\text { *Physical and } \\
\text { mathematical model to } \\
\text { examine systems }\end{array}$ \\
\hline
\end{tabular}




\begin{tabular}{|c|c|c|c|}
\hline 2004 & $\begin{array}{l}\text { Dinca-Panaitescu, } \\
\text { M. }\end{array}$ & $\begin{array}{l}\text { Cumulative Effects of } \\
\text { Chemical Spills Using a } \\
\text { Spatial-Temporal Dynamics } \\
\text { Analysis Algorithm }\end{array}$ & $\begin{array}{l}\text { *Spill modelling } \\
\text { *Algorithm to quantify } \\
\text { effects of chemical } \\
\text { spills }\end{array}$ \\
\hline 2005 & Tang K., N. M., & $\begin{array}{l}\text { A Land-Based Oil Spill } \\
\text { Management Planning } \\
\text { Framework for the Petroleum } \\
\text { Industry }\end{array}$ & $\begin{array}{l}* \text { Oil spill management } \\
\text { framework } \\
* \text { Statistical analysis }\end{array}$ \\
\hline 2007 & Han, H. Y. & $\begin{array}{l}\text { A Web-Based GIS Planning } \\
\text { Framework for Urban Oil Spill } \\
\text { Management }\end{array}$ & $\begin{array}{l}\text { *Statistical and spatial } \\
\text { analysis } \\
* \text { Oil spill management } \\
\text { planning framework }\end{array}$ \\
\hline 2010 & Cheng, V. & $\begin{array}{l}\text { The Development of } \\
\text { Risk-Based Spill Management } \\
\text { Criteria Related to the } \\
\text { Beneficial Use Impairments in } \\
\text { the St. Clair River }\end{array}$ & $\begin{array}{l}* \text { Statistical and risk } \\
\text { analysis }\end{array}$ \\
\hline 2013 & Cao, W. & $\begin{array}{l}\text { Probabilistic Spill Occurrence } \\
\text { Simulations and Quantitative } \\
\text { Water Quality Risk Analysis }\end{array}$ & $\begin{array}{l}\text { *Statistical and spatial } \\
\text { analysis } \\
\text { *Probabilistic } \\
\text { simulation of spills } \\
\text { *Quantitative Risk } \\
\text { Analysis } \\
\text { *Comprehensive } \\
\text { planning framework }\end{array}$ \\
\hline
\end{tabular}

Another comprehensive spill database that was reviewed is from the Energy Resources Conservation Board in Alberta, Canada. This database shares some of the same spill characteristics as with SAC spill data such as Cause Category, Substance and Volume Released, Environment affected, and Geographic Coordinates (Young, 2014). Other spill characteristics are specific to the oil and gas industry (e.g. Pipeline License Segment Identification, Pipe Damage Type) and rural areas (e.g. Affected Wildlife/Livestock). This is most likely due to the likelihood of many oil spills in Alberta occurring in rural 
areas and being associated with the oil and gas industry as well as the nature of the organization collecting the spill data (Young, 2014).

For more concise spill databases, many spill characteristics are also found in common with SAC database spill characteristics such as: Date, Identification Number, Substance Type, Location-Based Information, Quantity, Clean-Up Status, and Source (Northwest Territories Environment and Natural Resources, 2014; Shell Petroleum Development Company of Nigeria Ltd., 2014). The Hazardous Materials Spill Database for the Northwest Territories includes an additional column listing the agency associated with the clean-up of each spill. Examples include the Canadian Coast Guard, Environment Canada, and the Government of the Northwest Territories (Northwest Territories Environment and Natural Resources, 2014). An implication of this is the involvement of federal agencies, not just provincial, in spill management at the territorial level.

\subsection{Oil Spill Data Issues}

Collecting, recording, and analyzing spill data form the foundation for the development of a spill management framework. The creation of a geocoded spill database was deemed necessary to facilitate spill management plans from land spill research studies in the GTA (Li, 2005). Oil spill reporting can be considered to be one of the most important parts of an environmental management system. A heightened public awareness and more concern for the responsibility claimed for spill events have led to an increase in the reporting of increasingly smaller spills, though this is not necessarily reflective of an increase in the number of such incidents (Fingas et al., 2011). Useful information can be gained for a 
range of purposes such as forming policies, observing trends, and environmental enforcement and regulation (Tang, 2005).

The usefulness of spill reporting can be attributed to the accuracy of the reported data and whether the reporting of data is obligatory (Tang, 2005). Insufficient spill data and a lack of data consistency and follow-up data entry could decrease the usefulness of a spill database. It could also hamper the ability of municipal workers to understand spill causes, target mitigation measures, and develop preventative methods for oil pollution (Han, 2007).

A lack of frequent and proper spill data reporting can be a limiting factor for the development of a spill management plan. A recommendation was made by Cao (2013) for a longer history of sufficient spill data and information on spill duration. Improvements to the spill reporting system such as reporting or estimating spill duration time, identifying industrial North American Industry Classification System (NAICS) codes of chemical spills, and geocoding spill locations were also suggested (Cao, 2013).

The spill management planning framework that was developed was built upon the conceptual, comprehensive inland spill management planning framework previously proposed by Cao (2013). The approach developed by Cao (2013) was proposed as a proactive approach to address inland chemical spills. This approach has been further developed and tailored for municipal inland oil spills by transforming some previously 
suggested ideas into usable planning models or tools and by developing new planning models or tools.

Although previous research by Cao (2013) focused on chemical spills, the focus of the current research is on oil spills. Characteristic differences exist between the two types of spilled substances. Oils are often used to power and maintain machinery and are therefore used in a more generic way than chemicals. The tracking system for oils is also less stringent than for chemicals, making it more difficult to determine the type of oil involved in a spill - an important characteristic when modelling oil spills. However spilled oil can, in some ways, be easier to treat because it floats on top of water so it can be extracted, unlike spilled chemicals which could dissolve into water.

When spills occur near or along national boundaries, such as along the St. Clair River, international cooperation is required to ensure appropriate and effective preparedness, reporting, and response procedures between the involved countries (Cao, 2013). At the end of a study focusing on the St. Clair River, it was suggested that future research in spill management be based on improved spill data. It was determined that the spill records used did not contain information regarding the amount of material recovered after each spill and that such information could improve the accuracy of spill statistical analysis (Cheng, 2003). 


\subsection{Land Spills vs. Water Spills}

Amongst the authors of oil spill-related literature, it is commonly agreed that each oil spill should be addressed in a uniquely defined way according to the characteristics of the spill event itself. The characteristics of a spill event depend, to a large extent, on the behaviour of the spilled oil, which varies according to the environment in which it is spilled. The main difference in behaviour between oil that is spilled on land and oil that is spilled on water is the speed with which oil moves and the size of the impacted area (Owens, 2002).

Oil spilled on land moves much more slowly than oil spilled on water. The surface of the land is rarely flat and so the rate of downslope movement is a function of oil viscosity, air/ground temperatures, slope steepness, surface condition, precipitation and other weather conditions. The transport of oil on water is directed by winds and/or surface currents, rendering it much more difficult to predict the fate, behaviour, and effects of spills on water. Since the rate of movement of oil on land is slower and flow directions more simple than on open or coastal waters, the ability to predict the pathway taken by oil is enhanced. This allows response strategies for oil spills on land to be more accurately focused (Owens, 2002).

This assertion contradicts other research findings which maintain that the clean-up of land-based oil spills is a much more complicated process than the clean-up of marine spills because more variables have to be considered. For example, the movement of various types of oil in various habitats and spill site conditions. Examples of spill site 
conditions include soil type, soil moisture conditions, land slope, and the level and flow rate of groundwater (Kai, 2005). The temperature, the type and growth stage of vegetation present, and weather conditions such as snow or ice are other variables that must be taken into account. Adhesion properties of oil and its ability to permeate surface and subsurface environments are another consideration (Tang, 2005).

If oil reaches a waterway such as a river, the rate of transport may increase but the flow direction usually remains the same as the river currents which are essentially unidirectional. Oil and water are immiscible which results in a layer of oil above the water layer, making it easier to remove the oil. Nonetheless, floating oil on water can move very quickly so control and protection strategies may entail the identification of practical interception points and even the pre-staging or pre-deployment of equipment downstream to establish control and prevent spreading (Owens, 2002). This is why it has been stated that inland oil spills pose more challenges with respect to clean-up than marine oil spills (Tang, 2005).

Weathering, with respect to oil, is defined by the National Oceanic and Atmospheric Administration (NOAA, 2014) as:

The physical and chemical changes, such as evaporation, emulsification, and biodegradation, that oil or other pollutants undergo when exposed to the environment. Weathering can change an oil's density, viscosity (resistance to flow), rate of evaporation and dispersion into the water column, and the rate at which it may emulsify. A pollutant's weathering behaviour affects its eventual behaviour in the environment. 
The rates of different weathering processes depend upon the relative amount of surface area that is exposed to the spill. Since oil thins on water, these rates are expected to be slower for oil spills on land. Unlike oil on water, after oil spreads out on land, it reaches a stable condition in a short amount of time. Once this stable condition is achieved, the chance of further movement and weathering is at a minimum (Owens, 2002).

Consequently, the accuracy of forecasting and modeling the movement and weathering of spilled oil diminishes as oil moves from land to water environments. As a diverse range of scenarios are presented that must be considered, planning becomes more complex. Hence, preventing land spills from reaching water environments is a valuable response strategy. Most response strategies attempt to minimize the spread of spills by focusing on containment and control as near to the spill source as possible (Owens, 2002).

The underlying reasons for the similarities and differences between spills in land and water environments are differences in rates of spreading, mixing, transport, and weathering. As the scale of the response increases with the size of the affected area, the amount of oil recoverable significantly decreases. Some of the potential advantages and disadvantages of spills in land environments compared with spills in water environments are shown in Table 3. 
Table 3. Potential advantages and disadvantages of spills in land versus water environments.

\begin{tabular}{|c|c|}
\hline Advantages & Disadvantages \\
\hline $\begin{array}{l}\text { - The area impacted by the spill is } \\
\text { often relatively smaller in size. } \\
\text { - There is more potential for } \\
\text { predicting the movement and } \\
\text { effects of the spill. } \\
\text { - There are greater operational } \\
\text { opportunities and flexibility. } \\
\text { - There is greater potential for the } \\
\text { recovery of oil. }\end{array}$ & $\begin{array}{l}\text { The rates of weathering and natural } \\
\text { attenuation are slower. } \\
\text { - There is more potential for } \\
\text { affecting human-use activities and } \\
\text { resources. } \\
\text { - The potential for stricter standards } \\
\text { for clean-up and endpoints exists. }\end{array}$ \\
\hline
\end{tabular}

(Source: Adapted from Owens, 2002)

Regardless of the differences between spills in land and water environments, knowledge and operational practices can still be transferred from one environment to another (Owens, 2002).

\subsection{Oil Spill Impacts on Freshwater Environments}

Since land-based oil spills often spread into freshwater environments, a common source of drinking water for urban populations, it is important to review the resulting impacts and issues. Much of the research encountered to date has focused on the effects of oil spills on freshwater aquatic organisms which frequently suffer from toxicity when exposed to oil. In some cases, such as for oil-coated waterfowl (ducks, geese, or other large birds living in water), toxic effects may be very visible whereas in other cases, such as for benthos, a substantial amount of research may be required (Klerks et al., 2004).

Phytobenthos, a type of phytoplankton species, aid in regulating water quality in wetlands and are especially helpful in regards to phosphorus loading from urban runoff. An 
investigation into a sunflower oil spill in South Africa concluded that a decline in certain phytobenthos species occurred, producing a negative impact on phytoplankton species diversity. In cases of post-spill vegetable oil restoration activities, very high concentrations of fertilizers should not be applied to stimulate natural microbial activity for biodegradation of oil. If applied concentrations are too high, there is an increase in biomass and bloom formation of certain species which can threaten the food web structure and functioning of freshwater ecological systems (Oberholster et al., 2010).

Another study found that freshwater turtle behavior, water temperature preference, and home range size, are unaffected by crude oil exposure and rehabilitation. Oil-exposed freshwater turtles were successfully rehabilitated to their normal behavior in nature. Other uninvestigated areas of a freshwater turtle's biology could be affected by oil exposure, however, especially since turtles have long lifespans so some symptoms may not surface until several years later. A concluding recommendation of the study was that more research should be conducted on the long-term effects of oil exposure on freshwater turtle ecology (Saba \& Spotila, 2003).

Oil and its refined products are some of the most complex and variable mixtures to evaluate toxicologically since they are comprised of $75 \%$ short and long hydrocarbon chains (Castaldelli et al., 2012). If contaminant levels could be used to predict toxicity to aquatic organisms, then a quick analysis of hydrocarbon levels could be performed after an oil spill. This could provide a more effective area of focus for clean-up methods. Hydrocarbon measurements are routinely used as part of monitoring programs to assess 
oil spill impacts. In order to optimize environmental monitoring programs and to interpret their data, information on the relationships between various hydrocarbon measurements and toxicity are required (Klerks et al., 2004).

Regression techniques were used to determine the extent to which toxicity, as expressed by survival in bioassays, was related to various hydrocarbon measures. Approximately $60 \%$ of the variation in survival could be explained by the relationships between toxicity and hydrocarbon measures. Hence, it is still not feasible to quantify impacts at oil-contaminated freshwater sites unless actual toxicity measurements are conducted. Future research work should lead to the capability of predicting toxicity on the basis of chemical analyses which could be useful in developing more efficient clean-up procedures (Klerks et al., 2004).

Both petroleum and non-petroleum oil spills can produce negative effects on surrounding ecosystems, especially environmentally sensitive areas such as wetland ecosystems. The transformation of spilled oil in wetland areas is achieved through a variety of chemical, physical, and biological weathering processes. These processes change the composition, behavior, exposure pathways, and toxicity of spilled oil. Consequently, the environmental fate and toxicity of the transformed products may differ from that of the parent compounds (Oberholster et al., 2010).

A shortage of data on freshwater toxicity and toxicity interactions for petroleum hydrocarbons are obstacles for the development of freshwater models. As a result of the 
lack of models using detailed hydrocarbon information to predict toxicity of oil in freshwater ecosystems, the level of detail of the analysis provided by current analytical methods may be excessive (Klerks et al., 2004).

A lack of research in general, with respect to oil spills on freshwater wetlands, is a frequently made criticism. Only a few studies have addressed the multitude of factors that control the reaction of freshwater wetland ecosystems to oil contamination. This, in turn, limits the ability of researchers to predict the effects of a vegetable oil spill on freshwater wetlands, especially phytoplankton communities. Nonetheless, many studies have investigated the effects of vegetable oil on marine, river and coastal marsh environments (Oberholster, 2010).

A causal relationship has been established by numerous studies in Europe and the U.S. between aquatic pollution levels and diseases associated with fish. In one study, an assertion was made that chemical analysis can determine the extent of pollution but not the damage caused to organisms (Castaldelli et al., 2012). This assertion runs contradictory to the finding regarding chemical analyses from Klerks et al. (2003), though more work may have been done in this area since the latter study was completed.

The environmental quality of an array of aquatic habitats can be indicated by the occurrence of diseases and histo-cytological alterations in fish. Relevant data for these parameters are increasingly being used as biomarkers for studying oil spill impacts and aquatic toxicology. Breams, a type of fish, were captured after an oil spill into the Po 
River to detect the effects of oil contamination on ichthyofauna. Microscopic examination revealed the skin and gills of the fish, which were in constant contact with the water, were the most affected areas. Although all of the breams remained alive, these changes suggest that the release of oil was a stressful experience for the fish (Castaldelli et al., 2012). When compared with the previously discussed study regarding turtles, it seems that certain organisms may be more resilient to oil-exposed freshwater systems than others.

Freshwater environments damaged by oil spill events can be restored to provide fish-friendly habitats, as exemplified by a scenic Oregon watershed that was once impacted by a gasoline spill. In 1999, a tanker truck drove off of a road and spilled more than 5,000 gallons of gasoline into the Beaver Butte Creek. Chinook salmon, steelhead trout, and other fish and wildlife living downstream of the creek were affected. Plants were eliminated and the soil surrounding the creek became contaminated (NOAA, 2013).

A clean-up procedure began immediately and an assortment of restoration projects was selected to improve conditions for steelhead trout and Chinook salmon throughout the watershed. The first restoration project was carried out in 2011 and four more projects have been built since that time such as riparian fencing and road removal. More restoration projects will be conducted to provide the greatest benefit to injured fish and wildlife (NOAA, 2013). 


\subsection{The Three-Stage Framework: Prevention, Control and Response}

Oil spills, like other environmental emergencies, are unpredictable and can occur at any moment and under any kind of weather conditions. The relentless dependence by society on petroleum and its products combined with the innate tendency for human error and potential for equipment failure in producing, transporting, and storing petroleum, make oil spills almost inevitable (Fingas, 2011).

The conceptual three-stage comprehensive inland spill management planning framework proposed by Cao (2013), as previously mentioned, incorporated the three measures of prevention, control, and clean-up to address inland chemical spills. The framework entails a spill pollution prevention plan, a spill control plan, and an emergency response plan. The proposed planning framework was intended to assist municipalities in developing a spill management plan to prevent, control, and respond to inland chemical spills and to protect water resources. Technical planning tools were created to predict spill occurrence and associated water quality risk. These tools can help municipalities develop an inland spill management plan by identifying key spilled chemicals, responsible industries, and areas of concern using historical spill data analysis and predictive model simulations (Cao, 2013).

The spill pollution prevention plan (P2P) proposed by Cao (2013) should include spill data analysis, spill occurrence prediction, fate and transport in receiving waters, risk analysis of water quality impairment along the waters, and spill management. The structure of the probabilistic spill occurrence model is shown in Figure 5. 


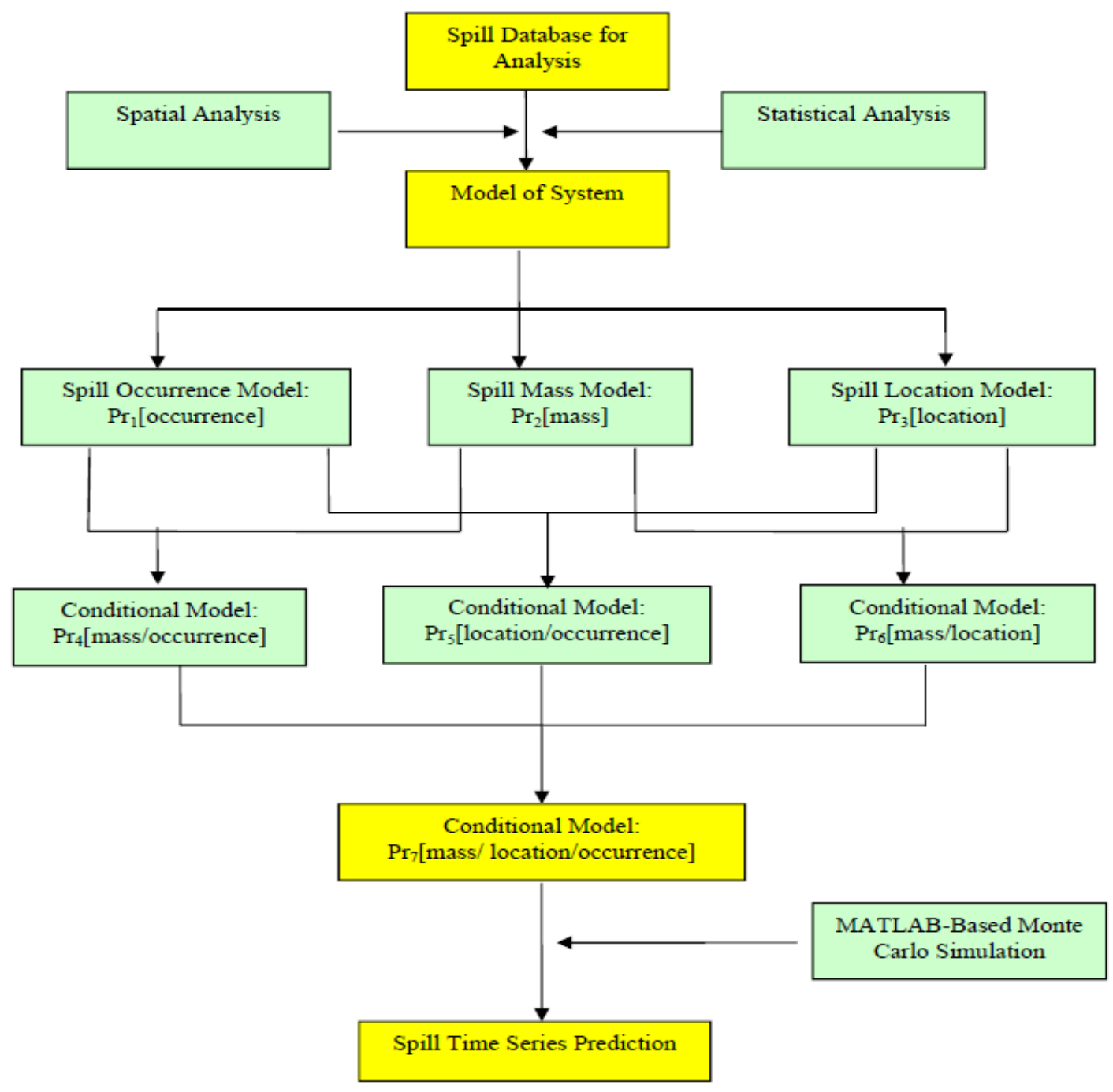

Figure 5. Structure of the probabilistic spill occurrence model. (Source: Cao, 2013)

A MATLAB-based Monte Carlo Simulation (MMCS) model was developed by Cao (2013), as shown next in Figure 6, to simulate the probabilistic quantifiable occurrences of inland chemical spills by time, magnitude, and NAICS code in a certain area. The MMCS model illustrated in Figure 6 contains variables M and TP which represent the number of simulation runs and simulation time period, respectively (Cao, 2013). 


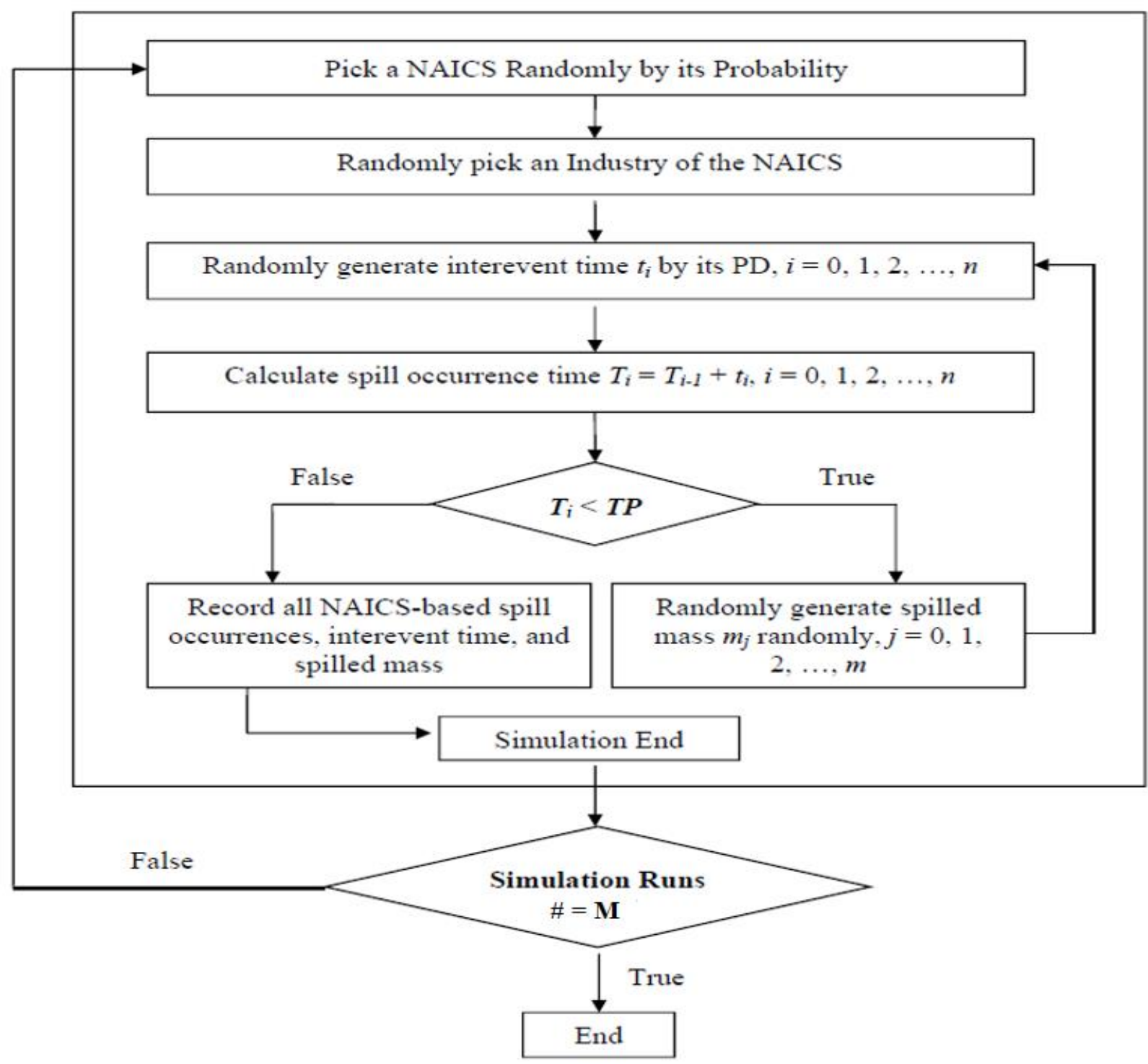

Figure 6. MATLAB-based Monte Carlo Simulation Model. (Source: Cao, 2013)

The goal of a P2P is to protect water quality and human health (Cao, 2013). An oil spill data analysis was conducted as part of the initial and subsequent stages of the research. Other suggested components of the proposed P2P that have been developed by Cao (2013) such as Geographic Information Systems (GIS) Mapping were also implemented or further developed in the current work. 
A management plan (MP) was also developed under the P2P to prevent the occurrences of inland spills at source. The proposed MP incorporates a range of programs including an Education Program, a Finance Program, a Collaboration and Cooperation Program, and an Inspection and Monitoring Program (Cao, 2013). An outline of each program is provided by Cao (2013) as well as clues for the development of a MP. The current work has expanded upon some of the clues that were proposed in the MP.

Although some of the ideas and suggestions put forth by Cao (2013) have been materialized in the current work, significant differences still exist. Spill management planning tools have generally been developed in a more quantifiable way in the current work for each stage of the three-stage planning framework, in order to assist urban municipalities facing spills. An example is the development of the prevention planning tool for enhanced employee training based on data analysis of a bivariate histogram.

Text or categorical variables have also been included in this study unlike in the previous work. The focus of the current work is also on spills of oil in contrast with spills of chemical which behave differently when spilled in water and are recorded with less stringency. The main difference, however, is that spill models and planning tools were developed for spills travelling on land as opposed to spills being transported by water. The focus of the current work has been on the land side of spill management as opposed to receiving waters. Cao's (2013) work was reviewed in the early stages of research and served to initiate some ideas with respect to predictive spill modelling rather than modelling inland spills based only on the analysis of historical data. 
A similar spill management planning framework was developed earlier for land-based oil spills in the petroleum industry and demonstrated at a petroleum company in Trinidad and Tobago (Tang, 2005). At the time of the development of the framework, there was no formal land-based oil spill management planning tool within the petroleum industry to address oil spill pollution. The framework is comprised of five components: oil spill characterization, prevention, control, clean-up, and economic evaluation. The middle three components are the same as the three stages specifically proposed in the current study. The same three stages are also put forth by Fingas et al. (2011) for both inland and marine oil spills and by Cao (2013) for inland chemical spills.

The beginning and ending components, spill characterization and economic evaluation, were the focus of the past study, as a result of the comprehensiveness of the planning framework (Tang, 2005). The planning framework developed for the current study also entails the initial component of oil spill characterization, primarily to develop prevention planning tools and as a preliminary stage of statistical analysis prior to planning tool development.

For the spill response and clean-up stage, oil spill characterization determines characteristics specific to each event since each event requires a relatively unique response. For example, the volume of oil spilled and the area that the spill covers may be the first steps in characterizing the event. These initial steps are important because the level of response is determined by the size and impact of an oil spill event (Fingas et al., 2011). 
In the oil spill management framework proposed by Tang (2005), the initial oil spill characterization stage identifies the trends and factors that influence oil spill events, which are then used to develop suitable oil spill prevention measures. An example is the development of a suitability matrix to identify preferred prevention measures that address specific oil pollution sources. Suggestions regarding other areas of oil spill prevention include ISO 14001 certification, oil spill contingency planning, and adherence to applicable environmental laws (Tang, 2005).

The suggested spill prevention measures were reiterated in another study based on a series of research projects to facilitate spill management planning in the GTA for oil and chemical spills. It was also suggested that cities should adopt sewer use by-laws, such as the Toronto sewer use by-law, which regulate industrial discharges to sewers and require industries to prepare pollution prevention plans ( $\mathrm{Li}, 2005)$.

In the study for the GTA, a spill management planning framework was developed for municipalities. Municipalities were advised to promote spill education and training by coordinating with industrial associations and provincial and federal agencies. Human error and equipment failure were found to be the main reasons for oil spills. Special emphasis should also be placed on employee training and preventative maintenance in the training programs of spill prone sectors such as the petroleum and transportation sectors (Li, 2005). This suggestion has materialized to some extent since intensive training programs have been developed to reduce the potential for human error (Fingas et al., 
2011). Spill prevention programs should also be designed and implemented for residents in relatively older residential areas and institutions still employing heating oil (Li, 2005).

The studies by Tang (2005) and Li (2005) both suggested that oil spill contingency planning can be conducted as a type of pollution prevention measure. Contingency planning has, however, been highlighted as a response measure for the control and clean-up phases of an oil spill management planning approach (Fingas et al., 2011). In this case, the focus of the contingency plan is on the treatment of the spill after it has occurred, rather than the prevention of the spill which contradicts the consideration of a contingency plan as a pollution prevention measure.

The type of contingency plan addressing control and cleanup can be customized for a certain facility. For example, if the contingency plan was devised for a bulk storage terminal, organizations and resources from neighbouring areas would be included which could lead to plans for spills of greater impact. Contingency plans for the various levels of government usually address the roles and responsibilities of responding personnel and outline how the response organizations will cooperate with one another. Such plans do not usually address specific response actions. When response actions are described in a contingency plan, they can be divided into the following phases: alerting and reporting, evaluation and mobilization, containment and recovery, disposal, and remediation or restoration. These phases can be applied to any type of facility and often overlap rather than occurring in sequence one-by-one (Fingas et al., 2011). 
Another generic planning framework was proposed for municipal oil spill management and demonstrated interactively using a Web-based GIS platform. The framework addresses prevention, control, response and cleanup, much like the other spill management strategies discussed. However, it provides for an oil spill inventory analysis stage as the initial stage. It is therefore comprised of four steps: (1) oil spill inventory analysis, (2) oil spill pollution prevention, (3) oil spill control measures, and (4) oil spill response and clean-up (Han, 2007).

The first step in the oil inventory analysis stage entails a data compilation process by recommending the data variables that should be recorded (Han, 2007). A data compilation process was also the initial step in the current research which was repeated and revised in later phases of the research. Preventative measures suggested in the generic planning framework were fairly similar to others discussed so far such as the adoption of a sewer use by-law. The initiation of programs such as sewer use control, water sampling, law enforcement, and education after adopting a sewer use by-law, was also advised to help protect municipal sewer use (Han, 2007).

It is evident that preventative measures for spill management plans usually encompass non-structural measures such as improved operational practices, education and training programs, and adoption of sewer use by-laws ( $\mathrm{Li}, 2005)$. The inclusion of structural measures within the preventative stage is a possible future research challenge. In the current framework, structural measures, as well as many of the suggested non-structural preventative measures for the prevention stage were included. 
Structural measures, such as spill control devices, are usually included in the control phase of a spill management framework, especially for industries. Examples of spill control devices include surface water ponds, containment walls, oil/water separators, and oil back-up systems. Containment walls are built structures that are usually situated around or near fixed operational structures that might be expected to produce a spill such as tanks or wells (Li, 2005). These systems confine any spills from the fixed structures within a certain area which saves the spilled oil and protects the surrounding environment from oil pollution. However, it must be ensured that there is proper drainage in order to drain rainfall and trap spills. Any captured oil can then be removed physically or mechanically (Li, 2005).

Another commonly used control device is the oil-water separator which intercepts spilled oil along the drainage systems and is based on the relative density of oil and water ( $\mathrm{Li}$, 2005). In the past, the City of Toronto recommended that businesses and industries practice pollution prevention by installing on-site oil separators; however the sizing criteria were not well-defined. The draft code practices put forth by the Canadian Petroleum Product Institute (1994) had also not yet been approved by the Ministry of the Environment at that time (Li, 2000; Chui, 2002).

An investigation was then undertaken to examine the possibility of installing oil-water separators at spill prone sewer outfalls. In order to apply oil-water separators at sewer outfalls, it was required that the devices be able to operate under high flow conditions and that the capacity be reflected by the land use characteristics of the corresponding 
watershed (Chui, 2002). An innovative spill control device was developed for the Humber Creek outfall. The Humber Creek is a tributary of the Humber River which is the main waterway of the Humber River Watershed which was selected as the case study area for the current research work (Chui, 2002).

A flow diversion structure was first designed to capture the dry weather flow at the outfall, which could then be transported into an oil-water separator. The oil-water separator was designed in accordance with the prescribed specifications in the manual of the American Petroleum Institute (1990). Next, a GIS-based analysis technique was used to identify potential treatment options for spill-prone sewer outfalls in the Town of Richmond Hill (Chui, 2002).

In another investigation, hydraulic oil spill control systems were examined in regards to transformer oil that is spilled under all weather and operating conditions at transformer stations. The focus of the study was on the design of oil trap systems and oil back-up systems at transformer stations. Oil trap systems capture spilled transformer oil and oil back-up systems provide an extra source of transformer oil after oil spills occur at a transformer station (Fan, 2004).

The study concluded that the oil trap system and the oil back-up system under examination were successful at preventing spilled transformer oil from entering the local sewer. It was recommended that a new physical model be constructed for the oil trap system to minimize the uncertainties in oil trap experiments. More numerical experimentation was recommended for the oil back-up system to ascertain the 
relationship between different volumes of spilled transformer oil and various design parameters (Fan, 2004).

On-site oil back-up systems and oil-water separators should be installed in commercial and industrial facilities that commonly experience oil spills. Food service and commercial facilities often release wastewater discharges that may include sediment, oils, and grease. An on-site oil and grease interceptor should be required in these facilities to prevent excessive amounts of oil and grease from entering the sanitary collection system (Han, 2007).

After a spill has occurred, on-site technologies control the quantity and quality of a spill to mitigate the negative impacts on the environment. For chemical spills, the characteristics of the spilled substance and the feasibility of the technology, both technically and financially, should be considered. Characterization and monitoring technologies such as gas chromatograph and mass spectrometry can be used (Cao, 2013). Other technologies such as remediation technologies have also been proposed, however, these technologies are usually considered to be part of the clean-up stage and are not usually included as part of the control plan.

Off-site spill control strategies and devices should also be considered for a spill prone watershed or area. A spill control volume should be included as part of stormwater management criteria for a spill prone area or watershed that is reflective of the statistical properties of the spill event volume of the facilities. A stormwater pond can be retrofitted for spill containment or an outfall oil/water interceptor can be installed at the downstream 
end of a spill prone sewershed (Han, 2007). Municipal capital programs and land use approval must be integrated with spill control strategies (Han, 2007). There must also be an emphasis on relevant regulations, acts, by-laws, or guidelines in a spill control plan. Since all measures used in controlling a spill at the source must be cost-effective, a cost analysis should be performed (Cao, 2013).

The final stage of an oil spill management framework is the response and clean-up stage. In the comprehensive three-stage inland chemical spill framework, an Emergency Response Plan (ERP) is proposed that consists of a Response Center, a Spill Clean-Up Plan, and a Potential Spill Plan. The ERP allows for a response team to be formed in advance to execute the spill response plan immediately when an event occurs. Three types of emergency response teams were proposed, as well as emergency response exercises for facilities along with emergency preparedness to foster international cooperation when a spill is shared by more than one country (Cao, 2013).

Education and training programs are integral to emergency preparedness. All staff and members of a spill prone area should be made aware of the risks of a spill and what actions to take in the event of a spill. More in-depth training can be provided to the personnel who will act as first responders in the event of an oil spill, which usually entails the personnel of local municipalities. A response team should therefore be established at the municipal level (Han, 2007).

The proper equipment to respond to a spill event should be placed in a spill prone area and made easily accessible. The placement of spill response resources should be a part of 
a spill management plan. The frequency and location of spill events can be analyzed to determine the type and amount of spill response equipment to be placed in certain locations. A Web-based GIS platform can be used to establish optimal locations for the placement of spill response equipment (Han, 2007).

A Web-based GIS platform can also be used to supply up-to-date information, an important element for any emergency situation. Maps of environmentally sensitive areas requiring special protection against spills can be generated by GIS. GIS can identify surface water intakes and map the stormwater drainage system with flow direction throughout the city. The shortest travel route to the oil spill for first responders and other spill response staff can also be determined using GIS (Han, 2007). Hence, there are many advantages to using a Web-based GIS platform.

After the first response to a spill event, a clean-up process must ensue. Only very small oil spill events may be exempt from some type of oil spill clean-up process. Careful selection of the clean-up techniques or technologies is important in order to mitigate the negative impacts on the surrounding environment to the fullest extent and to recover as much oil from the spill as possible. Various types of clean-up techniques or technologies exist for land surfaces such as sorbents, shallow trenches, manual removal, mechanical recovery removal, and in-situ burning (Tang, 2005).

Some of the techniques or technologies for oil spill recovery and clean-up that are the conventional and preferred methods are the physical response methods such as sorbents (in socks or booms), booms, berms, vacuums, skimmers, and physical herding. These 
methods are preferred because they usually do not require any kind of special conditional approval for their use. Other physical response methods include vegetation removal and cold and hot water flushing. Natural recovery, which entails leaving spilled oil on the spill site, is also considered a physical response method (Han, 2007). Some clean-up methods are more suitable for certain types of habitats than others (Tang, 2005).

After the clean-up phase, remediation measures must be implemented if the ecological integrity of the spill site prior to the spill has not been restored. The natural environment that has been adversely affected by the oil pollution can be rehabilitated by remediation technologies such as dispersants and gelling agents for oil spill contamination on water (Li, 2005; Tang, 2005). Such types of chemical and biological methods are often used with mechanical methods (Tang, 2005). It is believed to be impossible to remediate a site completely after an oil spill event, indicating the possibility for future research work in this area.

\subsection{Web-Based GIS}

The structure and advantages offered by merging the three-stage planning framework with a Web-based GIS system are exemplified by previous applications of Web-based GIS to areas of environmental monitoring and management. An example of a customized Web-based GIS application is given by the development of the first repository for Florida's wetlands which entailed an interactive Web-based GIS and a geodatabase. It provided a centralized storage centre and mechanism for sharing geospatial data, information, and maps of Florida's wetlands and adjacent agricultural ecosystems. It 
represents an attempt to address the environmental and political interest in the wetlands, especially the Everglades (Bloom et al., 2004).

The Web-based GIS system developed for Florida's wetlands, often referred to in the study as a Web-based GIS tool, a Web-based tool, or a Web-tool, consisted of map services, data services, meta data, and other information such as photographs. A Microsoft Access relational database was used to store and manage datasets. The data services were provided by the geodatabase that was developed. A three-tier client-server model was used to efficiently manage the database. Users were given the option to display the results from a database query in the Web browser on the client-side or download it to the local machine of the client (Bloom et al., 2004).

The Web-based GIS tool was created using Arc Internet Map Server (ArcIMS) software, a commercially available software product. ArcIMS was used to augment georeferenced point observations of soil attributes with other GIS data layers such as land use, geology, soils, and county boundaries. The final result offered users map display and interactive query, selection, and navigation functions (Bloom et al., 2004).

Components of the server-side included the ArcIMS application server, application server connectors, spatial server, and manager. The ArcIMS spatial server processes requests for maps and related information. The load of requests being sent is balanced by the ArcIMS application server which also tracks the type of map services that are running. The ArcIMS application server connectors are used to connect the Web server to the application server. Lastly, the ArcIMS manager is a suite of tools which provides access 
to all ArcIMS server-side functions and tools that were used to develop the application (Bloom et al., 2004).

The Web-based GIS tool developed demonstrated some of the general advantages for GIS such as the ability to easily share data globally and the provision of a cost-saving solution for users to access up-to-date spatial datasets. The spatial datasets were tailored towards a specific topic for users with limited GIS knowledge. After completion, state and regional agencies were some examples of authorities who were making increased use of the Web-based GIS tool to support decision-making in regards to the restoration and preservation of wetlands in Florida (Bloom et al., 2004).

Similarly, GIS can be used as a decision-support tool in cases which require immediate action by the proper authorities. For example, oil spills can have devastating effects on coastal habitats, especially if fast and appropriate response measures are not taken. All geographical information must be kept up-to-date for emergency purposes. Up-to-date information is also imperative for the operation of emergency response teams when establishing high priority areas (Milanelli et al., 2009).

A proposed system which demonstrated great potential for aiding emergency response teams employed a decision tree combined with a knowledge-based approach using GIS in order to assign oil sensitivity indices to Brazilian coastal habitats. Rules set by the official standards of the Brazilian Environment Federal Organ formed the basis of how the modelled system works (Milanelli et al., 2009). 
The sensitivity of an environment to oil spills mainly depends on the amount of impact the oil can exert on the area and on its persistence throughout the area. These factors will vary according to geomorphological characteristics such as substrate composition, land inclination, and degree of exposure to waves and tides. Geomorphological characteristics are seasonal and will vary throughout the year, an aspect that had not been accounted for in the preparation of Brazilian coastal sensitivity maps (Milanelli et al., 2009).

This gap in the Brazilian oil spill management framework provides an opportunity for GIS to act as a powerful resource. GIS overcomes the limitations of traditional sensitivity maps, such as being expensive to produce and usually being issued in paper form. These limitations hinder the ability of traditional sensitivity maps to be quickly distributed, transmitted, and updated. GIS enables the response to specific queries by promptly accessing precise, updated information while also providing spatial analysis capabilities (Milanelli et al., 2009).

An expert system is a computer system that simulates response measures which would be taken by an expert in a given situation within a particular area. The foundation of an expert system is knowledge which has already been input into the system. This knowledge is acquired either through experts in the field or from instructions and manuals. The acquired knowledge is transmitted to the system engine to generate rules used to rank Littoral Sensitivity Indices (LSI) for coastal habitats. The ranking rules are generated by using a decision tree, a method that has been successfully applied in other 
studies for the generation of GIS databases. The resulting information is then saved in a geographic database (Milanelli et al., 2009).

The expert system was developed to systemize oil spill environmental sensitivity using GIS. The output of the proposed GIS-generated expert system is Environmental Oil Spill Sensitivity (ESI) Maps. Since there is always some chance for an oil spill accident to occur, it is crucial to have an up-to-date classification of the oil spill environmental sensitivity of all habitats in regions where the petroleum industry is active or has the potential to become active (Milanelli et al., 2009).

The GIS-generated expert system demonstrated great potential for assisting emergency response teams in evaluating the damage from oil spills and implementing immediate solutions. The coupling of a geographic database and a knowledge-based GIS model of the expert system is similar to the approach carried out by the previous study which focused on the Florida wetlands (Milanelli et al.; Bloom et al., 2004). Both approaches are used as decision-making tools; however, the GIS-generated expert system is designed to aid in delivering fast responses and was not initially proposed as a Web-based GIS system (Milanelli et al., 2009).

Another area in which a Web-based GIS system has been suggested as a tool for environmental monitoring and management is sustainable agriculture, in which the purpose is to restore degraded cultivated soils. A prototype Web-based Decision Support System was developed for use in resource management and assessment of environmental quality in the agricultural sector. The designed Decision Support System (DSS) was 
aimed at being a part of the Conservation Reserve Program (CRP). The CRP is one of the largest programs of the United States Department of Agriculture (USDA). It strives to encourage farmers and ranchers to treat soil, water, and related natural resource issues on their lands in a manner that is environmentally sustainable (Awawdeh et al., 2007).

The prototype of the CRP-DSS was specifically intended for providing assistance to the USDA with respect to better management and planning of CRP enrolments. The CRP-DSS prototype was based on the same emerging industry-standard ArcIMS GIS platform which was used to create the Web-based GIS tool for the Florida wetlands. However, the platform integrated a mapping component Automated Feature Information Retrieval System (AFIRS) and a hydrological modelling component, Soil and Water Assessment Tool (SWAT). The AFIRS tool can be used for classifying image data using multi-source spatial data. The SWAT tool is used to simulate long-term trends of soil and water quality (Awawdeh et al., 2007).

A decision tree classification method was also used in this study. The method was used to combine both categorical and non-categorical data using remote sensing techniques. The CRP-DSS prototype which resulted was a single, seamless interactive system that fully integrated AFIRS and SWAT within ArcIMS. The integrated modelling system was designed to work as an efficient and effective tool for managing and evaluating the CRP. The system designed was only a prototype that was implemented in a distributed computing environment. Hopefully, the prototype system will shed some light on core 
issues necessary for the development of the operational CRP-DSS in the future (Awawdeh et al., 2007).

A prototype for a simple Web-based GIS urban oil spill application was developed to initiate the development of the comprehensive spill management framework for urban municipalities. The prototype demonstrates the application of a simple Web-based GIS platform in managing an oil spill database for Southern Ontario and in presenting relevant information on the Internet.

\subsection{Spill Modelling Software}

Methods which apply GIS technology have been used to model the movement and fate of oil spills on land and in surface water features. A system used to simulate land-based oil spills from pipelines and oil storage facilities has been used to model hundreds of spills in a range of terrains and environments throughout the world (Farrar et al., 2014). An

Overland Flow Toolset for the pipeline industry is available from an Environmental Systems and Research Institute (ESRI) Partner (a company in partnership with ESRI, the international supplier of GIS software). The spill model addresses spill movement of the substance through surface water and lateral spread over terrain (Environmental Systems and Research Institute [ESRI], 2017).

A few software programs are commercially available for modelling land-based spills. Since spill events can sometimes have extreme consequences, a land-based spill model should be as realistic as possible. A comprehensive spill model is based on proven algorithms which consider variations in terrain, fluid properties, surface cover, and soil 
absorption. The following is a list of fundamental parameters for modelling overland spills:

- Terrain or topography

- Drainage systems (natural or constructed)

- Fluid properties

- Operating conditions

- Potential release volume

- Potential physical travel paths

- Emergency response time

- Hydrography

- Surface cover

- Type of pavement

- Soil properties

- Weather conditions

- Season

- Time of day

- Crossings

- Crossings and transportation

- Hydraulic gradient

- Land use type

- Location

- Nearby built structures

- Nearby watercourses

Critical factors such as surface cover, soil properties, and weather conditions allow for flow resistance, soil absorption, and fluid evaporation to be included in the spill model. 
Weather conditions, for example, can have a dramatic effect on the speed at which the spill travels as well as the evaporative loss of the fluid. The above list is not exhaustive and more factors can be added.

The literature review has explored various facets and issues in regards to spill data and spill database development. The importance of proper spill database development for the development of spill management strategies has been emphasized and due consideration has been given to this aspect while developing the spill database for the current study. The investigation of the impacts of oil spills on freshwater environments highlighted the need for more and improved response and clean-up efforts with respect to site rehabilitation.

The review of Web-based GIS literature provided ideas for the development of the simple Web-based GIS prototype. The increasing implementation of the three-stage framework for spill management guided the current research in the direction of predictive spill simulation modelling rather than relying strictly on the analysis of historical spill data as was done in earlier work. The comparisons drawn between land spills and water spills, as well as the review of spill modelling software, aided in the development of the spill path and travel-time model. 


\section{Chapter 3: Methodology}

The overall methodology to develop the spill management framework and the associated models and planning tools is first presented, followed by a description of the compilation of the spill database and sub-databases. More details pertaining to the development of the transportation-related spill sub-database are given and the method of developing the highway spill model is described. The statistical treatment of the stationary spill data for both the stationary spill sub-database and the input simulation data set is then demonstrated. The method of analysis of the input simulation data set and the creation of the macro program is presented. The approach followed to devise the spill path and travel-time model is then discussed.

\subsection{Development of a Spill Management Framework}

The development of a comprehensive oil spill management planning framework is becoming increasingly important to prevent and mitigate the negative and harmful impacts of future oil spills. Prevention, control, and clean-up of oil spills are all important aspects of general oil spill management and were applied as part of a three-stage planning framework in the current study (Fingas et al., 2011). Many local municipalities explicitly include one or two of the three stages to address spills or oil spills. 
The methodology followed to develop a comprehensive three-stage inland oil spill management planning framework is comprised of four main parts as listed below and described in the following sections:

1) The compilation of the main spill database and two sub-databases: (1) A stationary spills sub-database and (2) A transportation-related spills sub-database.

2) The spatial analysis of transportation-related spills and the development of a highway spill model.

3) The development of a macro program to simulate or predict future spill scenarios and analyses of generated simulation data.

4) The development of an automated spill path and travel-time model.

The order of listed parts is not representative of the sequence in which each part was developed. Each part can be used as a model to generate planning tools which can be used to make recommendations for at least one or more of the three stages of prevention, control, and response and clean-up of inland oil spills. The methodology presented can be used as an approach that can be followed by a municipality or organization to develop their own customized spill management strategy. A general overview of the order of development is given by the flow diagram of Figure 7. 


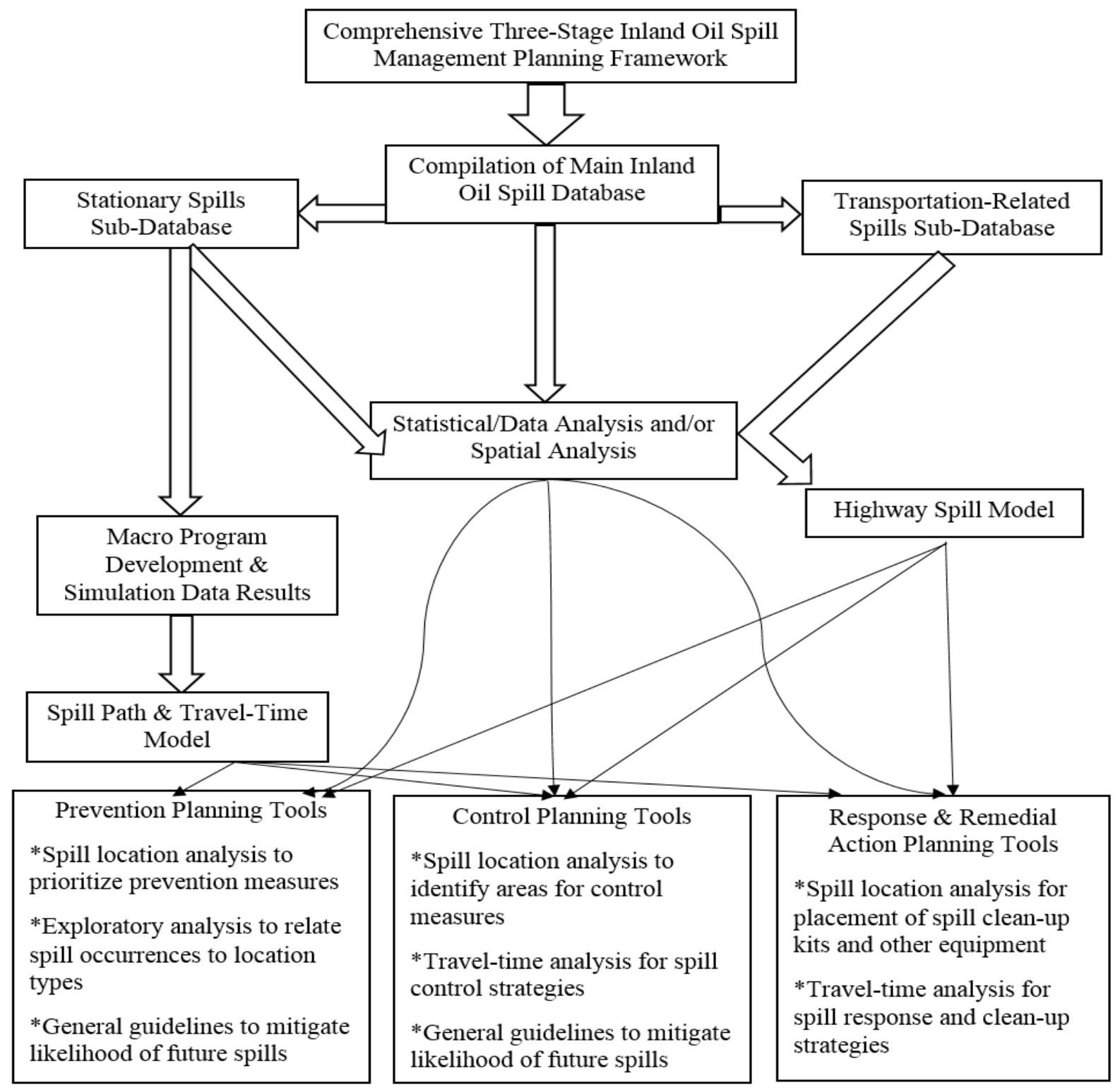

Figure 7. Flow diagram of general overview of the methodology.

\subsection{Compilation of Spill Database \& Spill Sub-databases}

A spill database was first developed to store all inland oil spill records, to analyze spill data and to create input data for model development. The main database comprising all on-land oil spills for Ontario was compiled from the following given data sets: 
1) A Microsoft Access Database developed from previous spill research at Ryerson University containing several tables including the first table of oil spill data for years 1988 to 2007 inclusive.

2) A spreadsheet containing worksheets for oil and chemical spill data for each year from 2003 through 2007, as supplied to Ryerson University by the Spills Action Centre (SAC).

3) Spreadsheets, provided by SAC, of oil and chemical spill data for each year from 2007 to 2013 inclusive.

Spill data beyond year 2013 could potentially be obtained from SAC but were not included as part of the range of spill data for the study.

The following additional data sets were also provided by SAC:

- An up-to-date spreadsheet for the group of spreadsheets covering years 2007 to 2013.

- A spreadsheet to update category names for the Sector/Source Type data column of 2013.

- A spreadsheet to confirm Contaminant Code values (values assigned to certain groups of oils) for various oils.

- A spreadsheet to fill in missing data for 2005 Contaminant Code values.

The first database initially given, resulting from previous spill research at Ryerson University, contains tables specific to chemical spills for 1988 to 2002, oil spills related to the train and hydro industries for 1999 to 2002 and a table of inland oil spill data for 
1988 to 2007. Since spills occurring within the chemical, train and hydro industries are not within the scope of the study and the focus was on inland oil spills, only the inland oil spill table in the database was used for spill database development.

The given Microsoft Access Database table for oil spills contains 26,896 oil spill records. Approximately 15,557 records from years 1988 to 2002 were extracted from the given database to develop the main spill database. The main spill database that was developed was slightly limited by the extracted table since it contains fewer data columns than the spreadsheets provided by SAC. The group of spreadsheets provided by SAC for years 2007 to 2013 contain raw spill data for a variety of fluid substances and a table of SAC

Material Group categories and Contaminant Code values, as shown in Table 4.

Table 4. SAC Material Group Categories and Contaminant Codes

\begin{tabular}{|c|c|c|c|}
\hline \multicolumn{4}{|c|}{ Material Codes } \\
\hline \multicolumn{2}{|r|}{ Oil } & \multicolumn{2}{|r|}{ Chemicals } \\
\hline 10 & Oil & 20 & Chemicals \\
\hline 11 & Crude & 21 & Acids \\
\hline 12 & Gasoline/Jet Fuel/Kerosene & 22 & Bases \\
\hline 13 & $\begin{array}{l}\text { Light Petroleum Oils (Motor, } \\
\text { Diesel, Furnace, Mineral) }\end{array}$ & 23 & Halogenated Solvents \\
\hline 14 & $\begin{array}{l}\text { Heavy Petroleum Oils (Bunker, } \\
\text { Lubricating, Tar, Asphalt) }\end{array}$ & 24 & Non-Halogenated Solvents \\
\hline 15 & Other Petroleum Oils & 25 & Pesticides \\
\hline \multirow[t]{3}{*}{16} & \multirow{3}{*}{ Non-Petroleum Oils } & 26 & PCBs \\
\hline & & 27 & Other Organic Chemicals \\
\hline & & 28 & Other Inorganic Chemicals \\
\hline \multicolumn{2}{|r|}{ Gases/Particulate } & \multicolumn{2}{|r|}{ Wastes } \\
\hline 30 & Gases/Particulate & 40 & Wastes \\
\hline 31 & Smoke & 41 & Liquid Industrial \\
\hline 32 & Dust Particulate & 42 & Liquid Hazardous \\
\hline
\end{tabular}




\begin{tabular}{|l|l|l|l|}
\hline 33 & Nitrous Oxides & 43 & Solid Non-Hazardous \\
\hline 34 & Sulphur Dioxide & 44 & Sewage \\
\hline 35 & Natural Gas & 45 & Agricultural \\
\hline 36 & Other Gases/Particulate & 46 & Other Wastes \\
\hline 37 & Heavy Particulate & \multicolumn{2}{|c|}{} \\
\hline 38 & Ozone Depleting Substances & \multicolumn{2}{|c|}{ Miscellaneous } \\
\hline \multirow{2}{*}{} & \multicolumn{3}{|}{} \\
\cline { 3 - 4 } & & \multicolumn{2}{|c|}{ Miscellaneous } \\
\cline { 3 - 4 } & 96 & Feed \& Foodstuff \\
\cline { 3 - 4 } & 97 & Not Applicable \\
\cline { 3 - 4 } & 98 & Unknown \\
\cline { 3 - 4 } & 99 & Other \\
\hline
\end{tabular}

Unlike the oil spill table from previous spill research, the spreadsheets of spill data provided by SAC contain spill records from all Material Group categories: Oil, Chemicals, Gases/Particulate, Wastes and Miscellaneous. A column of Contaminant Code values for each spill record is contained in each SAC spreadsheet. The extracted oil spill table from the earlier time period prior to 2003 was combined with SAC spreadsheet data from the later time period of 2007 to 2013 . Before combining the earlier spill research data with the more recent spreadsheet data, spill records from the recent spill data set were filtered specifically for oil spill records. All other types of substances or materials, such as chemicals and gases, were removed or excluded from the analysis.

Oils and oily substances are generally found within the Oils category as well as the Wastes and Miscellaneous categories. Data columns such Contaminant Name, Material Group and/or Contaminant Code, as well as others, were searched for spill records of oils or oily substances which were then transferred from the original spreadsheet to another spreadsheet. These extracted oil spill records from the SAC spreadsheets were then 
combined with the oil spill table into a larger, main spreadsheet based on common data columns.

Most significant data fields or columns under study are common to both the initial oil spill table and the SAC spreadsheets, although variations exist even amongst the annual SAC spreadsheets. The ordering of the column data also differs between the oil spill table and the SAC spreadsheets as well as from spreadsheet to spreadsheet. Assumptions regarding the selection and ordering of data columns within the final, main spreadsheet were based on this inherent variation of the given datasets.

The oil spill table was used as the template for the main spill database. However, since this table contained fewer data columns than the SAC spreadsheets, data columns or fields were added to earlier data from the oil spill table, such as the Contaminant Code column, to make it compatible with later data. Since there is only one data column containing date values in the oil spill table and two data columns in the SAC spreadsheets, Date Reported to MOE and Incident Date, the Date column in the oil spill table was assumed to be the incident date or the date on which the spill occurred. The incident date data from the oil spill table were then merged with incident date data of the SAC spreadsheets in the main spill database.

The oil spill table contains Latitude and Longitude columns of coordinate data for spill locations whereas the SAC spreadsheets contain coordinate data from the Universal Transverse Mercator (UTM) system, named Site UTM Easting and Site UTM Northing. 
The coordinate data from the two data sets were merged to form a pair of columns of coordinate system data, UTM Northing/Latitude and UTM Easting/Longitude, while maintaining coordinate values from each respective coordinate system.

The Road Type field lists various types of roads on which the spill event occurred or roads located near the spill event (e.g. arterial, collector, and highway). This field is unique to the oil spill table and the majority of data entries in this field were determined to be either blank or empty. Therefore, the Road Type field was not included in the main spill database.

The Location data column is also unique to the oil spill table and sometimes lists more specific on-site information than the Address field for each spill event such as service station, motor vehicle, plant and transformer. However, it contains fewer blank or unfilled entries than the Road Type field. The data categories listed under this field are fairly similar in meaning to the SAC Action Class column which also describes more specific on-site information such as land spills, watercourse spills and highway spills (usually highway accidents). The Location and SAC Action Class fields were merged to yield a supplementary data column known as Location/SAC Action Class in the main spill database.

A field named Source is also part of the oil spill table and also lists similar categorical data to the Location field to describe the source of a spill event such as motor vehicle, transport truck and pipeline. Another field called Sector describes the industry 
responsible for the spill such as transportation, petroleum, or government (municipal/provincial/federal). Within the SAC spreadsheets, however, a field named Sector/Source type entails categories of sources of spills with some category names referring to industrial sectors as well. For example, a Sector/Source type categorical data value of petroleum refinery states that the source of the spill is a petroleum refinery and indicates that the petroleum refinery falls within the petroleum sector. A Source data column was created in the main spill database as a combination of data from the Source field of 1988 to 2002 in conjunction with data from the Sector/Source type field of 2003 to 2013 .

The Sector field from the oil spill table and the Client type field (e.g. corporation, government, individual) from the SAC spreadsheets were used to create generic Sector and Client type fields within the main spill database. These fields, developed for the main spill database, describe the industry in which the spill occurred and the nature of the organization (e.g. private, public) or whether an individual is responsible for the spill. Data from the Company and Client fields of the two sets of data were also used to develop categories for the generic Sector and Client Type fields. Additionally, the NAICS (North American Industry Classification System) field, which is unique to the SAC spreadsheets, was also referred to for categorization of the more recent spill records.

The Report column of the oil spill table contains a description of the report given by the caller to SAC. The Summary column usually contains the name of the organization or company associated with the spill and a brief summary of the event. In the SAC 
spreadsheets, with the exception of years 2005 and 2006, there is just one field, Incident Summary, used to describe or summarize the spill event. The Report and Summary field data for each spill record in the oil spill table and in the SAC spreadsheets for years 2005 and 2006 were merged and aligned with Incident Summary field data to form a single column of one to three sentences of details summarizing each spill event.

Any remaining data columns common to both the oil spill table and SAC spreadsheets were implemented in the main spill database. These data columns are listed next along with corresponding examples:

- Address: street address or closest intersection of streets

- Region/Site District Office: City of Toronto, Algoma, York, Durham

- Municipality/Site Municipality: City of Toronto (North York), Howland Township

- Oil Type: gasoline, diesel, furnace oil, crude oil, motor oil, tar, asphalt, vegetable oil

- Volume (Spill Volume)

- Unit (original unit assigned to spill volume - for example, Litres)

- Cause (Answers the question, "What kind of spill happened?".)

- Reason (Answers the question, "Why did the spill happen?".)

- Impact/Nature of impact: soil contamination, surface water pollution, air pollution - \% Clean-Up (percentage of spill cleaned up) 
After synthesizing all of the column or field data, the process of extracting and removing spill records not within the scope of research was conducted. Text filtering techniques were used to select records pertaining to spill events considered as exclusions, such as real-time emergency responses and extreme events including fires and disasters. Spills occurring at major hydro stations were removed while spills entailing local pad-mounted or pole-mounted transformers were retained. Spills involving transport by train, aircraft or watercraft, unless at marine terminals, were removed.

Out-of-province spills originating from locations outside the boundaries of the Province of Ontario were also removed. Moving or standing vehicles that travel using the roadway system were included in the main spill database such as cars, trucks and street sweepers. However, other vehicle types that may produce spills or be involved in spill events in other areas, such as construction vehicles and farm tractors were excluded.

The Impact or Nature of Impact fields contain categories that refer to the medium or media impacted by the spill such as surface water, soil or air. Although many spills impact multiple mediums, spill events with no impact on inland surface waters were excluded such as spills that only contaminate soil or cause vegetation damage. All spill events, unless otherwise stated, were assumed to impact inland surface waters, either directly by travelling to a river or watercourse or indirectly through the storm sewer system. This assumption was applied to blank or unclear entries within the Impact or Nature of Impact data columns. All excluded or removed records were placed in a separate spreadsheet and are referred to as "other" spill types. 
The development of the main spill database revealed the myriad of circumstances under which an inland oil spill event can occur. As previously described, inland oil spill events can occur at fixed locations or due to moving vehicles or transport-related circumstances. For instance, the improper transport and delivery of oil by trucks can result in very large volumes of oil being spilled from containment devices within the truck onto roadways and surrounding areas. Transport trucks carrying non-oil or other goods can also cause sizable on-land oil spills due to collisions resulting in damaged fuel tanks.

In order to characterize various spill events and tailor the development of tools or models to certain kinds of events, the spill data was divided into oil spills occurring at fixed locations and spills occurring in relation to transport. Keywords were used to search through the Incident Summary and Report columns in addition to other data columns to categorize spill events as either stationary or transportation-related.

Three fields were added to the spreadsheet regarding the categorization of each spill record. The first field assigned a decision to the record as to whether to retain the record or move it to another database or spreadsheet for transportation-related spills or other excluded oil spill event types. The second field expanded on this decision to explain why the spill record was being retained or removed. The third column categorized the spill as either stationary, transportation-related, or other. A spreadsheet or sub-database was developed for each spill category. The stationary and transportation-related or mobile 
sub-databases were further cleaned and edited to use as input data for statistical analysis and model development.

An Interevent Time (days) column was added to the stationary and transportation-related sub-databases to calculate the number of days in between spill events. Another column was later added when the data was used as input to the macro program. The additional Interevent Time (days) column provided an optional data column to eliminate values of zero days when fitting distributions to this variable. Although the minimum interevent time (IET) possible between spill events is zero days, zero values may influence the selection of a distribution for the variable that does not accurately represent the true distribution of the values. For certain statistical methods, it was assumed that Interevent Time values, in days, are greater than zero.

To prepare the data for geocoding, location data such as address, region and municipality were cleaned, edited and revised. Many of the municipalities were associated with incorrect regions which were corrected and updated to reflect more accurate and current regional classifications according to the Association of Municipalities Ontario (AMO). To distinguish original data from revised data, new or additional data columns were added to represent the revised data fields.

Additional data columns were added with respect to the original Oil Type field. The wide range of oil types were condensed and organized into detailed Oil Type categories based on the SAC Material Code groupings shown in Table 4. Entries within the 
Contaminant Code field were then corrected and updated to reflect revised oil type categories. Fields were added to convert Spill Volume (L) to Spill Mass (kg) for each spill record based on density values for each kind of oil.

For the stationary spill sub-database, a minimum spill event volume of $6 \mathrm{~L}$ (approximately $6 \mathrm{~kg}$ for some oil types), was used to exclude very minor spill events from subsequent analyses. Therefore spill events with spill masses $(\mathrm{kg})$ of less than $6 \mathrm{~kg}$ were removed since these events can often be cleaned up very easily. The many Spill Volume (L) entries in the stationary spill data set which contain blank or zero values were assumed to be errors representing non-trivial events. The non-trivial events were assumed to contain spill event masses $(\mathrm{kg})$ of at least $6 \mathrm{~kg}$ and were included in the main spill database. This assumption is realistic since every spill reported must be associated with a non-zero spill mass $(\mathrm{kg})$ quantity.

After excluding minor spill events and establishing the Spill Mass (kg) column, the minimum spill mass of $6 \mathrm{~kg}$ was added to each blank or zero value in the Spill Mass $(\mathrm{kg})$ data column to eliminate entries with blank or zero values. The same approach was used for the transportation-related or mobile spill sub-database as zero spill masses $(\mathrm{kg})$ or blank entries were replaced with an approximate minimum value, although very small or trivial spills were still retained for this database.

An additional data column for $\%$ Clean-Up values was appended to each of the stationary and transportation-related sub-databases after adding a value of one percent to each 
unfilled data entry or entry with a zero percent clean-up value. This provided an optional column of non-zero \% Clean-Up data values for statistical techniques, when required. The addition of the data column was based on the assumption that many of the zero values were recorded erroneously or that perhaps the issue of percent clean-up was not addressed during the call to report the spill.

The NAICS Code column of the given data contains very few entries that are filled, which led to the enhancement of the NAICS Code field or fields for both stationary and transportation-related spill data sets. NAICS Code entries in the original data set are mainly recorded as categorical variables as the title of the NAICS Code category. Any numeric entries are usually listed in the form of numeric codes such as the NAICS Canadian Industries six-digit code. Categorical data fields, in both text and numeric form, for each level of the NAICS code hierarchy were developed within the stationary spill data set. NAICS code fields ranged from the NAICS Canadian Industries detailed six-digit code to the more general NAICS Sector two-digit code.

The three-digit NAICS Subsector code was the only NAICS Code that was developed in the transportation-related spill sub-database. Other categorical variable fields within the transportation-related spill sub-database are of the same type and name as the stationary spill sub-database, however the categories are slightly different with the exception of the Nature of Impact spill variable. Categories of the transportation-related spill categorical variables were customized for the data while maintaining similar categories to the stationary spill sub-database since both data sets are very similar. Transportation-related 
spill variables with different sets or kinds of categories as compared with stationary spill categorical variables are: Source Type, Sector, Client Type, Cause and Reason. The spill database was imported into the ArcGIS mapping software program, ArcMap, at various stages to geocode the address or location data. Latitude and Longitude values were then added as fields to each sub-database after geocoding to further verify and specify the spill location. However, the external spill database was not permanently connected to ArcGIS.

\subsection{Transportation-Related Spill Sub-Database \& Highway Spill Model Development}

After close inspection of the data, an assumption was made to form the basis of spill analysis for the transportation-related spill sub-database. Transportation-related spills occurring as a result of collisions or accidents were assumed to be related to the type of road or to the design of the road. These kinds of spills were classified as "road-related vehicle accidents/collisions" while other spills related to vehicular issues or external issues, assumed to be unrelated to road design, were classified as "vehicle-related incidents". A collision is defined by the Transportation Association of Canada (TAC, 2007) as:

An event in which travel by a vehicle results in the vehicle being in an inappropriate location, or in an inappropriate location at an inappropriate time, culminating in unwanted contact with a fixed object, vehicle, other roadway user or other obstacle.

Vehicle-related incidents are mainly related to vehicular functioning or other external factors not associated with the road system such as adverse road or weather conditions. 
Although some vehicle-related incidents may be related to road-related issues such as the cleanliness of the road, the issue of concern is associated with road maintenance rather than road type or road design.

The Cause field was re-categorized accordingly to reflect the focus of the mobile spill analysis by classifying spill records as either road-related vehicle collision or vehicle-related incidents. Road-related vehicle collisions were extracted for the City of Toronto from the entire data set of all road-related vehicle collisions in Toronto. The City of Toronto eventually became the area of focus or study area for mobile spill analysis.

The mobile or transportation-related spill sub-database was first geocoded using ArcGIS to produce a shapefile of transportation-related spill points that occurred in Ontario. The geocoded data set of mobile spill points represent historical incident data points of inland oil spill events in which the focus is on the presence or absence of the spill event instead of a measured attribute associated with each point (Environmental Systems Research Institute [ESRI], 2017).

The incident data points or oil spill points were mapped as clusters using an optimized version of a hot spot analysis technique that identifies statistically significant spatial clusters of high values (hot spots) and low values (cold spots). Optimized hot spot analysis creates maps of statistically significant hot and cold spots by calculating the Getis-Ord Gi* (pronounced G-i-star) statistic for each feature in a data set (ESRI, 2017). 
The Getis-Ord local statistic is given as $G i^{*}$ which is calculated as:

$$
G i *=\frac{\sum_{j=1}^{n} w_{i, j} x_{j}-\bar{X} \sum_{j=1}^{n} w_{i, j}}{S \sqrt{\frac{\left[n \sum_{j=1}^{n} w_{i, j}^{2}-\left(\sum_{j=1}^{n} w_{i, j}\right)^{2}\right]}{n-1}}}
$$

where $x_{j}$ is the attribute value for feature $j, w_{i, j}$ is the spatial weight between feature $i$ and $j, n$ is equal to the total number of features and:

$$
\begin{array}{r}
\bar{X}=\frac{\sum_{j=1}^{n} x_{j}}{n} \\
S=\sqrt{\frac{\sum_{j=1}^{n} x_{j}^{2}}{n}-(\bar{X})^{2}}
\end{array}
$$

The Gi* statistic returned for each feature in the data set is a z-score, therefore no further calculations are required (ESRI, 2017). A new output feature class is created with a Z-score (Gi* statistic), p-level and confidence level bin (Gi_bin) for each feature in the input feature class. A field named NNeighbors is also included stating the number of neighbours included in the calculations for each feature (ESRI, 2017).

The z-scores and p-values tell you where features with either high or low Gi* values cluster spatially by looking at each feature within the context of neighbouring features. A feature with a high $\mathrm{Gi}^{*}$ value or z-score may be interesting but may not be a statistically significant hot spot because it is not surrounded by other features with high values as well (ESRI, 2017). For statistically significant positive Gi* values or z-scores, the larger the $\mathrm{Gi}^{*}$ value or z-score is, the more intense the clustering of high values is to form a hot spot. For statistically significant negative Gi* values or z-scores, the smaller the Gi* 
value or z-score is, the more intense the clustering of low values is to form a cold spot (ESRI, 2017).

The method employed by the ArcGIS tool to generate optimized hot spot analysis results, is given in the following general steps:

1) Initial data assessment,

2) Aggregation of incident data,

3) Identification of an appropriate scale of analysis,

4) Calculation of the Getis-Ord Gi* statistic,

5) Creation of the output features.

The colours of the grid cells of the map output represent hot and cold spots (ESRI, 2017).

In this study, characteristics of the input point feature class for transportation-related spills were evaluated to produce optimal hot spot analysis results (ESRI, 2017). Hot spot analyses were performed to analyze the spatial pattern of clusters of mobile spill points to determine if there were any statistically significant clusters. The optimized version of hot spot analysis evaluated the characteristics of the input feature class of transportation-related spill points to produce a map of statistically significant hot and cold spots for optimal results (ESRI, 2017).

The tool used for performing optimized hot spot analyses was applied to both sets of spills points for Ontario and for the City of Toronto. Both sets of spill points were representative of all oil types. Based on the results, recommendations were made for spill 
management strategies. Organizations can also follow the same approach to develop their own spill management strategies.

A highway spill model was developed by selecting spill points from the shapefile of transportation-related spill points within Toronto, which are within 50 metres of major highways. A buffer distance of 50 metres was selected to ensure that the selected spill points would be sufficiently close to the highways of interest to be able to associate the spill events with the road type category of highways. The drainage channel alongside highways is usually approximately $5 \mathrm{~m}$ in width. Therefore, some of the spills that were not recorded as being directly on a highway but very close to a highway were assumed to have originally occurred on a highway. It was then assumed that these spills travelled into a nearby ditch or slightly further away in which case they could still be classified as highway spills.

A shapefile was then created with the selected points and the number of spills occurring within the specified distance of 50 metres within major Toronto highways was analyzed. The selected spill points were analyzed by editing the attribute file of the shapefile to append a field for classifying the spill point. Each spill point was classified as occurring at or near an interchange location (including spills occurring on interchange ramps), spills occurring at non-interchange locations such as along a section of the highway and spills occurring on ramps that are not interchange ramps. Multiple spills occurring at the exact same location with non-specific address data were excluded from the analyses and classified as unknown due to limited spill site address data. Multiple spills occurring at 
the exact same point with specific spill site address data were assumed to be spills which occur at the same general location and were classified accordingly.

\subsection{The Development of a Web-Based GIS Prototype}

A prototype of a simple Web-based GIS urban oil spill application was developed in the current study, using ArcGIS for Server 10.1 software, to initiate the development of a Web-based GIS three-stage planning framework. A sample of data containing only transportation-related and stationary spills was extracted from the spill database. The data was then cleansed, simplified, and geocoded to produce map layers using ArcMap 10.1 software. Separate map services for stationary and transportation-related spills were developed such as displaying inland oil spill locations and providing relevant information for each inland oil spill.

The prototype was developed using the ArcGIS for Server 10.1 (Windows) software program which makes geographic information available to others in an organization and/or anyone with access to the Internet. A GIS resource such as a map or a globe provided on ArcGIS for Server is called a service. The geographic information is made available through web services which allow a server computer to receive and process requests for information sent by other devices.

At first, a sample of inland oil spill data from the original Microsoft Access spill database (1988-2002), containing both transportation-related and stationary spills, was cleansed, simplified, and geocoded to produce map layers using ArcMap 10.1 software. Using the map layers, separate map services for stationary and transportation-related spills were 
created. Additional data layers, such as polygon municipal boundaries, were then added to oil spill point layers to make the maps more useful as well as other mapping functions.

An ArcGIS Server site is composed of a GIS server, a Web Adaptor, a Web server and a data server. The design of these four elements comprise the ArcGIS for Server site architecture which depends on factors that determine the type of deployment scenario selected such as the purpose of the site, the extent of the distribution of the site, and the security level of the site. A one-machine deployment scenario was selected for the prototype because it is suitable for development and prototype purposes while maintaining a reasonable level of security. The steps followed to configure a one-machine deployment scenario were:

1) Install ArcGIS for Server,

2) Log in to ArcGIS for Server Manager and create a site,

3) Install and configure the ArcGIS Web Adaptor (IIS),

4) Connect to the GIS server in ArcGIS for Desktop,

5) Grant permissions to data directories,

6) Register data,

7) Publish services, and

8) Use the services. 
The one-machine deployment scenario exposes one GIS server through the Web Adaptor. For this prototype, the client and the server resided on the same computer, restricting accessibility and manageability of the site by someone with access to the computer.

\subsection{Statistical Treatment of Stationary Spill Data}

\subsubsection{Stationary Spill Sub-Database}

After the sub-databases were initially compiled, an investigation was undertaken to examine any relationships between the numeric and non-numeric variables under study. A correlation matrix, which indicates the similarity and closeness between objects was developed to determine the correlation coefficients between each variable and all other variables (TIBCO Software Inc. [StatSoft], 2017).

A multiple linear regression was performed using each of the three numeric variables as the dependent variable and the remaining numeric and non-numeric variables as the predictor variables. Both standard and stepwise regression analyses were performed. Attempts were made to transform variables in order to improve regression results.

Lastly, the most popular or common oil type was selected and a multiple linear regression was performed using a stepwise forward method as previously done.

A principal components and classification analysis (PCA), was carried out to detect the underlying structure of the data. The first analysis considered 12 variables, both numeric and non-numeric, as active variables and no supplementary variables. Many variables within the data set were analyzed because the technique is intended for large-sized 
problems. The PCA technique attempts to fit straight lines that best fit the clouds of points in the vector spaces of variables and cases based on the least squares criterion (StatSoft, 2017). The PCA technique is not part of the macro program that was subsequently developed to simulate future spill data but is a statistical technique used to investigate the multivariate data of the stationary spill sub-database.

Some experiments involved each version of each variable as input, such as all five variables representing each level of the NAICS code hierarchy. Smaller numbers of qualitative variables were sometimes used to speed up the iterative process and to evaluate the difference in results from larger numbers of qualitative variables. The maximum number of iterations used for the NIPALS algorithm was 50 and the convergence criterion was 0.0001 . The number of components was automatically fitted using a cross-validation technique.

A recipe-like, step-by-step data mining approach was also used to build predictive models to relate the target or dependent quantity of Spill Mass $(\mathrm{kg})$ to the remaining input independent or predictor variables. The input data set consisted of 2,330 stationary records. In the first step, the input data was cleaned and prepared for modelling. In the next step, data analysis, statistical analyses were conducted on both the input and target variables.

Various statistics were calculated on the continuous variables (categorical variables are excluded from such an analysis) such as mean, standard deviation, skewness, kurtosis and 
observed minimum and maximum values. A simple random sampling technique was used to create a validation sample. Code names from the validation sample were used for the training and validation or testing samples.

The next stage of data mining entailed the calculation of descriptive statistics for all continuous variables. The descriptive statistics include mean, standard deviation, skewness, kurtosis, and the observed minimum and maximum values. The data redundancy step was selected to eliminate highly redundant predictors using the correlation coefficient method with a specified criterion value of 0.8 . Redundant predictors which were highly correlated $(\mathrm{r} \geq 0.8)$ were eliminated.

After the data cleaning and preprocessing steps were completed, an advanced feature selection strategy was implemented to extract a subset of 12 input variables that are most likely related to the target variable. Three models were then built for the selected input variables and "tried" against the data (StatSoft, 2017). The three methods used both the training and testing data subsamples: (1) Boosted Trees, (2) C \& RT (a classification tree program), and (3) Neural Network. All three models were deployed to predict the target variable of spill mass $(\mathrm{kg})$.

\subsubsection{Input Simulation Data}

An input simulation data set of stationary spills was developed to perform simulations of predicted future spill records using the macro program that was created. Some data analyses were performed to develop planning tools such as the illustration of 
relationships between categorical variables. The correlation between numeric input variables was also investigated.

Gasoline spill records for the Humber River Watershed were extracted from the data set

of gasoline spills in Ontario. A shapefile was created to display and analyze the extracted spill points for the case study area. A map was created of the spill points in the case study area. Some geographic analyses were performed to investigate the spatial characteristics of the spill data set.

For instance, a spatial statistical analysis technique known as Grouping Analysis was carried out to group features based on feature attributes such as Spill Mass (kg), \% Clean-Up, and optional spatial constraints of Longitude and Latitude. A variety of trials were conducted to determine the optimal number of groups, variables and spatial constraints to group or cluster the points. The results, in addition to limitations imposed by given GIS data, were used to re-evaluate the study area for the purpose of spill model development. The case study area was then reduced to the lower or southern part of the Humber River Watershed which lies within the City of Toronto.

\subsection{Macro Development \& Simulation Data}

The input simulation data set still retained the original data set of gasolines spills within the Humber River Watershed for the purposes of macro simulation. Descriptive statistics and a correlation matrix were calculated for the numeric variables of the input simulation data. To investigate the relations between pairs of categorical variables, bivariate 
histograms were produced and analyzed. Recommendations and suggestions for spill management solutions were made based on some of the bivariate histograms. The same approach can be taken for any spill data set under study.

The spill data set containing all gasoline spills for Ontario was used in an earlier edition of the Excel macro program to determine the minimum number of simulations necessary to repeat the system of loops until the number of spills predicted in each results spreadsheet stabilized. The minimum number of simulations was then used to further develop the macro program to only repeat the loop system for the pre-determined minimum number of times. An aggregate spreadsheet was later developed as a unit of output from the macro to compile all results from all simulation results spreadsheets. Descriptive statistics were performed on the variables or characteristics of the aggregate spreadsheet data.

The Excel macro program developed was used to simulate future on-land oil spill scenarios based on historical spill data. The macro language used to develop the spill prediction model program was the Statistica Visual Basic (SVB) language. SVB integrates the industry standard Visual Basic programming language into the Statistica software program. Statistica Visual Basic is very similar to Microsoft Visual Basic Version 6 but not entirely the same as it contributes thousands of additional new functions to the standard syntax of Microsoft Visual Basic (StatSoft, 2017). 
A log of analyses is first recorded by the macro program to select the "best-fitting distribution" for each variable and to perform simulations based on the best-fitting distribution of each variable. The log records the type of module or tool run and the corresponding options and variables selected. For the stationary spill input dataset, the best-fitting distribution was determined for five numeric variables and eight non-numeric or text variables. Each row of data within the input dataset is treated as a distinct case or spill record by the module. Cases were selected by the program based on the completeness of the spill record. Therefore, cases with missing information for any one of the thirteen numeric and non-numeric variables were excluded from the analysis.

The module fitted continuous distributions for numeric variables and discrete distributions for non-numeric variables. Based on distribution summary statistics, distributions were ranked according to best fit from highest to lowest. The best-fitting distribution was selected based on this ranking and a priori analysis for each variable which entailed the fitting of distribution curves to histograms. By overlaying various types of distribution curves onto histograms for each variable, a priori analyses revealed which distribution most closely resembled the shape of the histogram of the variable. The distribution which most closely resembled the shape of the histogram and the ranking of the distributions from the module output were used to pre-select the best-fitting distribution for each variable.

Once the best-fitting distribution was pre-selected, custom settings were applied for the distribution of each numeric variable to specify any applicable restrictions. Simulations 
of predicted values for each variable, both numeric and non-numeric, are derived from the best-fitting distribution for each variable. Restrictions and customized settings were applied to generate realistic and sensible predicted values within the simulation results. For example, a minimum value of $0 \%$ and a maximum value of $100 \%$ are specified for the \% Clean-Up variable to apply restrictions to the range of predictable percentage values.

Likewise, minimum values for the distributions of the Interevent Time (days) and Spill Mass $(\mathrm{kg})$ variables were truncated at zero or positive values close to zero to simulate non-negative values in the results. Minimum and maximum values were not specified for the two remaining numeric variables, Latitude and Longitude. Nonetheless, minimum and maximum values of each variable are intrinsically incorporated into the workings of the module regardless of specifications or alterations made by the program developer.

After the bulk of the recorded portion of the macro was fully customized, a system of loops was created within the macro to generate simulation results spreadsheets based on the distributions of best fit for each variable. A separate results spreadsheet is generated for each simulation and the number of simulations is represented by an adjustable variable within the macro prior to the looping system.

Each simulation generates a results spreadsheet as a series of spill data for predicted spill scenarios occurring within one of two approximate timeframes: a 10-year planning horizon or a 20-year planning horizon. Spill records or cases are generated sequentially 
for the time period of the planning horizon using one of two simulation methods: Monte Carlo or Iman Conover. Each row within the simulated results spreadsheet is a case or spill record, similar to the rows and cases of the historical spill data set which serve as input data for the macro program that is referenced at the start of the macro program. The Monte Carlo simulation method was used to ignore the correlation structure of the input data set when generating simulation data while the Iman Conover simulation method incorporated the correlation structure. However, the Monte Carlo simulation method can be applied to incorporate the correlation between variables. Input data sets containing variables exhibiting dependence between one another can therefore be included.

Within a given simulation results spreadsheet, each case or spill record is assigned a number and each column is a list of predicted values for the variable over the approximate planning horizon. A singular row or case is comprised of predicted values for all numeric and non-numeric variables as well as additional data generated internally within the loop structure of the macro. Additional columns which round and then sum Interevent Time (days) values are used to signal an exit to the main and outermost loops. The predicted Interevent Time (days) values within a column are rounded in a neighbouring column and then summed in a subsequent column to calculate the time period over which the series of spill data approximates. If the summation of rounded Interevent Time (days) values approximates the first planning horizon of 10 years, the main loop is exited and recommences to generate another results spreadsheet data set. 
The cycle repeats itself for as many simulations as initially specified to produce the required number of simulation results spreadsheets for each planning time period.

After producing the required number of simulation results spreadsheets for the first planning horizon of 10-15 years, the process is repeated for the longer planning horizon of 20-25 years. Other data columns added within the loop system of the macro provide column statistics such as the mean and standard deviation for the variables of Interevent Time (days), Spill Mass (kg) and \% Clean-Up. A column with a single entry for the number of spill records is also given.

Since each row or spill record represents one predicted spill, the number of spill records is equivalent to the number of spills predicted for one simulation or one series of spill data. Remaining data columns include Latitude and Longitude values as well as text label values for non-numeric variables. The text label values are numeric codes that represent discrete categories for each predicted non-numeric category text label.

The aggregation of column statistics is also performed by the macro program. The mean and standard deviation for each of the three main numeric variables is listed in a summary table in addition to the number of spills for each simulated spill data set. Graphs are then plotted for each column in the summary table with table values displayed as $y$-values against the number of the simulation being performed as the $\mathrm{x}$-value. The process of aggregating results is repeated twice for each collective set of data generated for each planning horizon time period. 
A section was added towards the end of the main loop to compile all results from all simulation results spreadsheets into an aggregate data set. The results from each simulation spreadsheet were compiled into an aggregate results spreadsheet by establishing a very large, blank spreadsheet and copying the results from each individual simulation results spreadsheet into the larger spreadsheet. After running the program, a final spreadsheet is produced containing all of the results from all simulations stored in columns of continuous data.

A bootstrapping component was added to the latter half of the macro program to estimate sampling distributions for statistics calculated on random samples selected from the simulation results spreadsheets. The bootstrapping component was based on the concept of bootstrapping that is presented for the bootstrapping module of the statistical software program. The bootstrapping component developed for the macro program was created independently from the embedded bootstrapping module or tool of the software program. As a result, there is no recorded portion of SVB code for bootstrapping that is part of the component that was developed.

Once a results spreadsheet was generated from a simulation (also known as a replication), a random sample of half of the cases was drawn from it and stored in a separate spreadsheet. A spreadsheet containing spill records of the random sample is associated with the corresponding simulation results spreadsheet from which it was extracted. Within the bootstrapping section of the macro program, a loop was used to round the sampled Interevent Time (days) values before calculating various statistics. Since it was 
not necessary to calculate the time period for a random sample, the sampled IET (days) values were not summed to calculate a time period as was carried out for the simulation results spreadsheets. Bootstrapping cannot be used to perform predictive data simulations in place of the Monte Carlo simulation method because it is sampling from the results spreadsheets which are the predicted spill data sets. It is not sampling from the historical input data set to generate new data.

A recorded portion of a tool was then implemented to calculate a variety of basic descriptive statistics for the three main numeric variables: (1) Interevent Time (days), (2) \% Clean-Up, and (3) Spill Mass (kg), for all random sample spreadsheets. A summary of the statistics was then displayed in a spreadsheet. Histograms were also created for each of the three main numeric variables with normal distributions fitted on top for comparison. The entire output of the macro program developed consists of the following:

- spreadsheets of simulation results and random samples from bootstrapping

- summary tables and graphs of simulation results

- aggregate spreadsheets of compiled simulations results

- a summary of descriptive statistics for random samples from bootstrapping

- histograms with fitted normal distributions for the three main numeric variables from the statistical summary of bootstrapping

The output of the macro program contains comprehensive data and analyses for predicted future spill data. 


\subsection{Spill Path and Travel-Time Model Development}

The flow of oil over land is guided by the physical nature of the land surface and the degree of slope over which it flows. After the oil enters a surface water feature, its flow is guided by surface currents and a different modelling approach is required (Farrar et al., 2014). An oil spill can be assumed to enter the storm sewer system first before reaching a surface water feature in an urban environment. In this study, the oil spill path was modeled for both the overland and storm sewer system components.

The flow path of an on-land urban oil spill is usually comprised of two sequential stages: 1) The first stage entails the starting point of the spill path which is the initial location of the spill, referred to in this study as the "spill point". The spill usually continues from the spill point to an entry point or opening of the stormwater-collection system. It is assumed that the entry point is the access point of the stormwater-collection system, also known as the access hole, manhole, sewer receptacle or clean-out structure (Chin, 2013).

2) The second stage includes the path from the access point or sewer receptacle to the exit point of the storm sewer system, which is assumed to be an outlet structure such as an outfall.

Another assumption of the spill path and travel-time model is that the surface area over which the spilled oil flows consists of paved, impervious area that typically dominates the urban landscape. Pervious area such as grass is therefore excluded from the spill path and travel-time model though the natural terrain is used in ArcGIS to calculate the 
overland path of the spill. Due to the assumption of imperviousness, oil absorption into pervious areas such as grass or soil is excluded when calculating overland flow time.

To model the spill path, an application was used within ArcGIS to automate the entire spill pathway by calculating the travel-time for each stage of the flow path: first the overland spill path, followed by the storm sewer system spill path. As the spill travels in the downslope direction over the land surface, the velocity $(\mathrm{m} / \mathrm{s}), \mathrm{V}$, of the leading edge of the spill is determined by the slope of the land surface using Manning's Equation with constant n:

$$
\mathrm{V}=(1 / \mathrm{n}) \mathrm{R}^{2 / 3} \mathrm{~S}^{1 / 2}(\mathrm{~m} / \mathrm{s})
$$

where $\mathrm{R}$ is the hydraulic radius, $\mathrm{S}$ is the slope, and $\mathrm{n}$ is a dimensionless number that characterizes flow resistance (Farrar et al., 2014). Assuming $\mathrm{n}$ is 0.05 and $\mathrm{R}$ is $0.122 \mathrm{~m}$ :

$$
\mathrm{V}=4.92 \mathrm{~S}^{1 / 2}(\mathrm{~m} / \mathrm{s})(5) .
$$

Equation (5) can be used to calculate the velocity $(\mathrm{m} / \mathrm{s})$ of a spill travelling overland. If the length of the overland path is known, $\mathrm{L}(\mathrm{m})$, the flow time, $\mathrm{t}(\mathrm{s})$, can be estimated by:

$$
\mathrm{t}=\mathrm{L} / \mathrm{V}(\mathrm{s})(6) \text {. }
$$

For the overland spill path, cost distance analysis was used to create the least-cost path or corridor from the spill point to the access hole or sewer receptacle for direct entry into the sewer system. To determine the length $(\mathrm{m})$ of the overland pathway of an oil spill, a cost distance analysis was performed using cost distance analysis tools within ArcGIS. Cost distance analysis explores the movement of a travelling object over a landscape and is 
usually used to create the least-cost path or corridor between a source and a destination (ESRI, 2017).

The cost to move from one raster grid cell to another is influenced by the following three characteristics:

1) The surface characteristics (the cost surface).

2) The characteristics of the moving object, which could include the mode of travel such as walking or driving.

3) The characteristics of the movement when moving from one raster grid cell to another, such as moving upslope or downslope.

The general cost distance formula is described by the following equation: cost $=$ cost of travel over surface $*$ characteristics of the mover $*$ movement characteristics (7) on the surface (ESRI, 2017).

The surface characteristics are incorporated by the cost distance tools using a cost surface which is usually a digital elevation model (DEM). The cost surface is a raster layer which defines the impedance or cost to move planimetrically through each cell. The values of the cost raster can be integer or floating point but they cannot be zero or negative because you cannot have a negative or zero cost (ESRI, 2017).

All cost distance tools use the same algorithm to calculate an output raster in which each cell is assigned the accumulative cost to the closest destination cell which, in this study, is the sewer point or sewer receptacle. The algorithm employs the node and link cell 
representation used in graph theory in which the centre of a cell is considered a node and each node is connected to adjacent nodes by multiple links. Every link has an associated impedance which is derived from the costs associated with the cells at the end of each link (based on the cost surface) and from the direction of movement through the cells. Each cell is assigned a cost that represents the cost per unit distance for moving through the cell. The final value for each cell is the size of the cell multiplied by the cost value. For example, if the cost raster has a cell size of 20 and that particular cell has a cost value of 5 the final cost of a cell is 100 units (ESRI, 2017).

After the overland spill path is delineated and measured, the pathway through the storm sewer system must be determined. For the spill path within the storm sewer system, a geometric network was used to model and trace the downstream spill path from the point of entry at the sewer receptacle to the exit point at the outfall. A geometric network was developed using ArcGIS to model the storm sewer system. A geometric network can be used to model other common networks and infrastructure systems such as water distribution, electrical lines, gas pipelines, telephone services and water flow in a stream. It is essentially a set of connected edges (lines) and junctions (points). Connectivity rules are applied to the edges and junctions to represent and model the behaviour of the network or infrastructure system (ESRI, 2017).

The ModelBuilder application was used in ArcGIS to develop the model. This application develops models by connecting series of geoprocessing tools in a chain-like manner so that the output of a preceding tool is used as input for the following tool 
(ESRI, 2017). Pre-processed input is required, however, for some of the tools that initiate the model or for connecting branches of the model. To first establish the model, appropriate environment settings were applied and a connection was made to two file geodatabases that were created for the storage of input and output data sets. Flow diagrams which illustrate the entire model are shown in Appendix A.1 as three separate sections or parts due to the breadth of the diagram.

Data sets stored within the input file geodatabase were prepared in advance and used as input for some of the initiating tools of the model while data sets stored in the output file geodatabase were generated by tools running within the model. The first tool of the model is a cost distance tool which receives two prepared input data sets: (1) the sewer receptacle points of the study area and (2) the given digital elevation model (DEM) of the City of Toronto. The original format of these two data sets and a data set of the sewer system lines within the City of Toronto, were provided by the municipal government and modified to suit the purposes of this research and the model. The given DEM encompasses the entire City of Toronto with a resolution of 3 metres by 3 metres. The XY Coordinate System is the North American Datum 1927 Transverse Mercator (NAD 1927 Transverse Mercator). It was assumed that the DEM was derived from LiDAR data, therefore the vertical accuracy is approximately $\pm 30 \mathrm{~cm}$ ("A Survey and Clarification of DEM Vertical Accuracy", 2019).

Since the DEM was considered to be the central and limiting piece of GIS data, all other GIS data sets were projected to the common coordinate system of North American 
Datum 1927 Universal Transverse Mercator Zone 17 North (NAD 1927 UTM Zone

$17 \mathrm{~N})$. Modifications were made to the DEM to make it usable as model input, including the conversion of the units of the elevation values to metres which is the linear unit of the given data file. Any sinks or areas of internal drainage greater than 1 metre were then filled in to remove small imperfections within the data (ESRI, 2017).

The shapefiles for sewer receptacle points and storm sewer system lines were initially given within a projected custom coordinate system named MTM_3Degree. As a result, they were projected from the custom coordinate system to the selected common coordinate system. New shapefiles were then derived based on selecting records pertaining specifically to the storm sewer system, as compared with other kinds of sewer systems such as sanitary and combined sewer systems. Sewer receptacle points and storm sewer system lines falling within the southern region or lower basin of the Humber River Watershed were then extracted to create sewer receptacle point and storm sewer system line shapefiles for the study area.

Both sets of shapefiles were also enhanced by adding coordinate values to the attribute tables using geoprocessing tools. For the storm sewer system lines shapefile, the modified DEM was used to interpolate z-coordinate values thereby making it z-aware. Coordinate values for $\mathrm{x}-, \mathrm{y}-$, and $\mathrm{z}$-coordinates were then added as the beginning, middle and ending coordinate points for each storm sewer line segment. The length of each storm sewer line segment was determined and the slope of each segment was then calculated. 
According to the majority of pipeline segment records within the attribute table, the pipeline segments were assumed to be round and made of concrete. Full-flow conditions were also assumed for pipe flow, therefore the depth of flow was taken to be the diameter of each pipe segment. Based on these assumptions and the given diameter (m) values of each pipeline segment, the flow area $\left(\mathrm{m}^{2}\right)$ and flow rate $\left(\mathrm{m}^{3} / \mathrm{s}\right)$ were calculated for each storm sewer line segment. The flow area $\left(\mathrm{m}^{2}\right)$ is the circular, cross-sectional area of the pipeline segment which was calculated using the diameter $(\mathrm{m})$ of each segment. The flow rate, $\mathrm{Q}\left(\mathrm{m}^{3} / \mathrm{s}\right)$, was calculated for each pipeline segment using the final expression of Manning's equation for full-flow conditions as shown in equation (8) (Chin, 2013):

$$
Q=\frac{1}{n} A R^{\frac{2}{3}} S_{0}^{\frac{1}{2}}=\frac{1}{n}\left(\frac{\pi D^{2}}{4}\right)\left(\frac{D}{4}\right)^{\frac{2}{3}} S_{0}^{\frac{1}{2}}=\frac{0.312}{n} D^{\frac{8}{3}} S_{0}^{\frac{1}{2}}
$$

where $n$ is the Manning roughness coefficient in storm sewers, $A\left(\mathrm{~m}^{2}\right)$ is the flow area of the pipe, $R(\mathrm{~m})$ is the hydraulic radius of the flow area, $D(\mathrm{~m})$ is the diameter of each pipe segment and $S_{0}$ is the slope of the pipe (dimensionless). When using equation (8) for concrete pipes in storm sewers, a Manning's $n$ of 0.013 is a common assumption. The travel-time, $t$, in seconds and in minutes, of each oil spill through each pipe segment was then pre-calculated for each segment for later use and referral using equation (6).

A wide range of geoprocessing tools were then linked together to create an inland spill path model consisting of three main parts as shown in Appendix A.1. The first part of the model entails two cost distance tools. The first cost distance tool receives the DEM and the sewer receptacle points shapefile of the study area, which were prepared in advance, 
as input. Using the sewer receptacle points shapefile as the input source locations and the DEM as the input cost raster, two output raster datasets are generated: the output backlink raster and the output cost distance raster (ESRI, 2017).

These two raster datasets are received as input, in addition to a shapefile of predicted spill points within the study area that was also prepared in advance, by a least-cost path tool. The shapefile of predicted spill points is derived from a data set of predicted spill cases that was generated by the macro developed for simulating future spill events. It is a simulation results spreadsheet which was converted into a points shapefile and projected into the common coordinate system.

The two raster datasets and the shapefile of predicted spill points are transferred to the least-cost path tool and used to generate an output polyline shapefile that delineates the least-cost spill paths. The output polyline shapefile is the key output for the entire model as it shows the least-cost paths from the source locations or spill point locations to the destination access points or target points of the storm sewer system. The attribute table associated with this output shapefile lists the least-cost value and length of each path as well as an identification number for the destination feature at the end of each path.

The second, intermediate part of the model modifies the output polyline shapefile by making it z-aware to enable it to receive z-elevation values. New fields are then added to the attribute table to calculate the overland slope for each spill pathway. The overland slope values are then used to calculate overland velocity $(\mathrm{m} / \mathrm{s})$ and travel-time $(\mathrm{s})$ for each 
corresponding spill along each spill pathway. The additional data and calculations are derived using geoprocessing tools that employ Python 9.3 and Visual Basic (VB) calculation expressions.

The travel-time calculation column may list " $<$ Null $>$ " values when division by zero is encountered due to zero velocity values which stem from zero overland slope values. To convert this text entry into a numeric, usable form, geoprocessing tools are then used to calculate the minimum travel-time within the travel-time column and replace the " $<$ Null $>$ " values with the minimum value.

After the overland travel-time is calculated for each spill, the end point of each line feature representing the spill path is converted into a point feature class. The converted point feature class is then attached to or "snapped" to the underlying coincident sewer receptacle point class to form a new feature class of points. The points within the new feature class of points are used as starting points or "flags" in the third, final portion of the spill model.

The third part of the spill model entails the creation and development of a geometric network to trace the spill pathway within the sewer system. A separate data set was first created within the input file geodatabase to contain the storm sewer line and sewer receptacle point feature classes that were already prepared in advance, for the development of the geometric network. These two feature classes are used to create the geometric network using a geometric network tool to connect the storm sewer lines to the 
sewer receptacle points. Constraints on the types of network features which may be connected to one another are imposed through network connectivity rules.

The two kinds of network connectivity rules available, the edge-junction rule and the edge-edge rule, are imposed one after the other using tools specific to these functions. The first network connectivity rule, the edge-junction rule, establishes the connection between the edge feature class, the storm sewer lines, and the junction feature class, the sewer receptacles. The second connectivity rule, the edge-edge rule, establishes the connection between the edge feature class, the storm sewer lines, and itself, the storm sewer lines, through a connection with the junction feature class, the sewer receptacles (ESRI, 2017).

The flow direction for the geometric network is then set based on the digitized direction which enables the flow direction within the pipelines to be established along the digitized direction of the edges. The downstream paths within the sewer lines are then traced based on the starting points which were created by the new feature class of flag points, as mentioned earlier.

The network analysis problem of tracing the downstream paths of the oil spills through the storm sewer system is solved based on the flags as starting points to create a group layer that stores the results of the trace as a selected set of layers. The layers involved in the trace, such as the sewer receptacles (including a separate default junction layer) and the sewer lines, are then extracted and made into feature layers using two more 
geoprocessing tools. Lastly, another tool is used to connect coincident endpoints of the traced sewer line segments and sum the pre-calculated travel-time values to provide a more concise table of output travel-times for spill paths within the storm sewer system. The travel-times for the storm sewer system spill paths can be summed with the overland travel-time values to yield the total travel-time, in minutes or in seconds, of the entire spill path.

The overall methodology developed can be applied to other regions or jurisdictions outside of the case study areas and the City of Toronto. First, a spill database must be compiled using local land-based spill data. The data should then be cleaned, edited and subdivided according to any categories of interest. Some statistical and exploratory data analyses is then performed to investigate the characteristics of the data, determine the correlation structure and develop spill management planning tools.

The macro program is used to simulate future spill data based on past spill data using a simulation method which either ignores or incorporates the correlation structure. The main requirement for the macro simulation model is to assign best-fitting distributions which reflect the histograms of the input data variables and the distributions proposed by the results of the program. The predicted spill data can then be plotted and analyzed using spatial analysis techniques to develop more planning tools. Lastly, the spill path and travel-time model can be applied using the DEM and the storm sewer pipe and sewer points shapefile which are specific to the given area. 


\section{Chapter 4: Results \& Discussion}

In this chapter, the results from each section of the methodology are discussed. First, the results from the analysis of the main inland oil spill database are discussed. Next, the results from the spatial analysis of the transportation-related spill data and highway spill model development are examined. The outcome of the development of the Web-based GIS prototype is then presented and reviewed. A statistical analysis of the results stemming from the macro simulation program and corresponding input and output simulation data is given. Lastly, the intermediate and end results of the spill path and travel-time model are investigated.

\subsection{Analysis of Main Inland Oil Spill Database}

The literature review of various spill databases demonstrates the requirement for improved data collecting, recording, and reporting. Many of the data entries in the given SAC spill database were left blank. There are many errors and inconsistences, even amongst entries pertaining to the same spill record. As a result, it is difficult to interpret the variables or characteristics of many spill records and to analyze data. Over time, there has been a noticeable improvement in terms of data accuracy, clarity, and consistency as seen from later data of 2007 to 2013 compared with earlier data of 1988 to 2006.

An improved system of spill data collection and recording is recommended. The benefit of the improved data sets of later years is the ability to work with the data with greater 
efficiency and clarity. The drawback to this improvement is a reduction in the level of detail and transparency of spill records. For example, the more recent spill data sets contain one column for a summary of the spill incident which generally has a brief description of the occurrence while the earliest data set contains two columns, one for a summary of the event and another for the report given by the caller. The summaries and reports given in the earlier data are usually quite detailed and descriptive, especially when compared with the terse incident summaries of later data.

The initial, main inland oil spill database consists of extracted inland oil spill records from the spill database of previous research (1988-2007) and the given SAC spreadsheets (2003-2013). It contains all on-land oil spill records before further subdivision into transportation-related and stationary spill sub-databases. As previously mentioned, many spill characteristics stored in the initial main database are the same characteristics found within the given spill data except for Interevent Time (days). Interevent Time (days) is derived from the dates of the spill events and is a measure of the time, in days, between two consecutive spill events.

After the development of the two spill sub-databases, spill characteristics were re-organized into fewer numbers of categories based on commonality and significance. The initial main spill database therefore maintained the multitude of individual categories for each categorical variable as shown in the original data sets. Preliminary analyses of initial spill database characteristics revealed a high number of blank, unspecified or unknown category values for the Cause, Reason and Nature of Impact fields as illustrated 
in the bar charts of Figures 8 and 9. This provides further evidence to support the recommendation for improved spill data collection, recording and reporting.

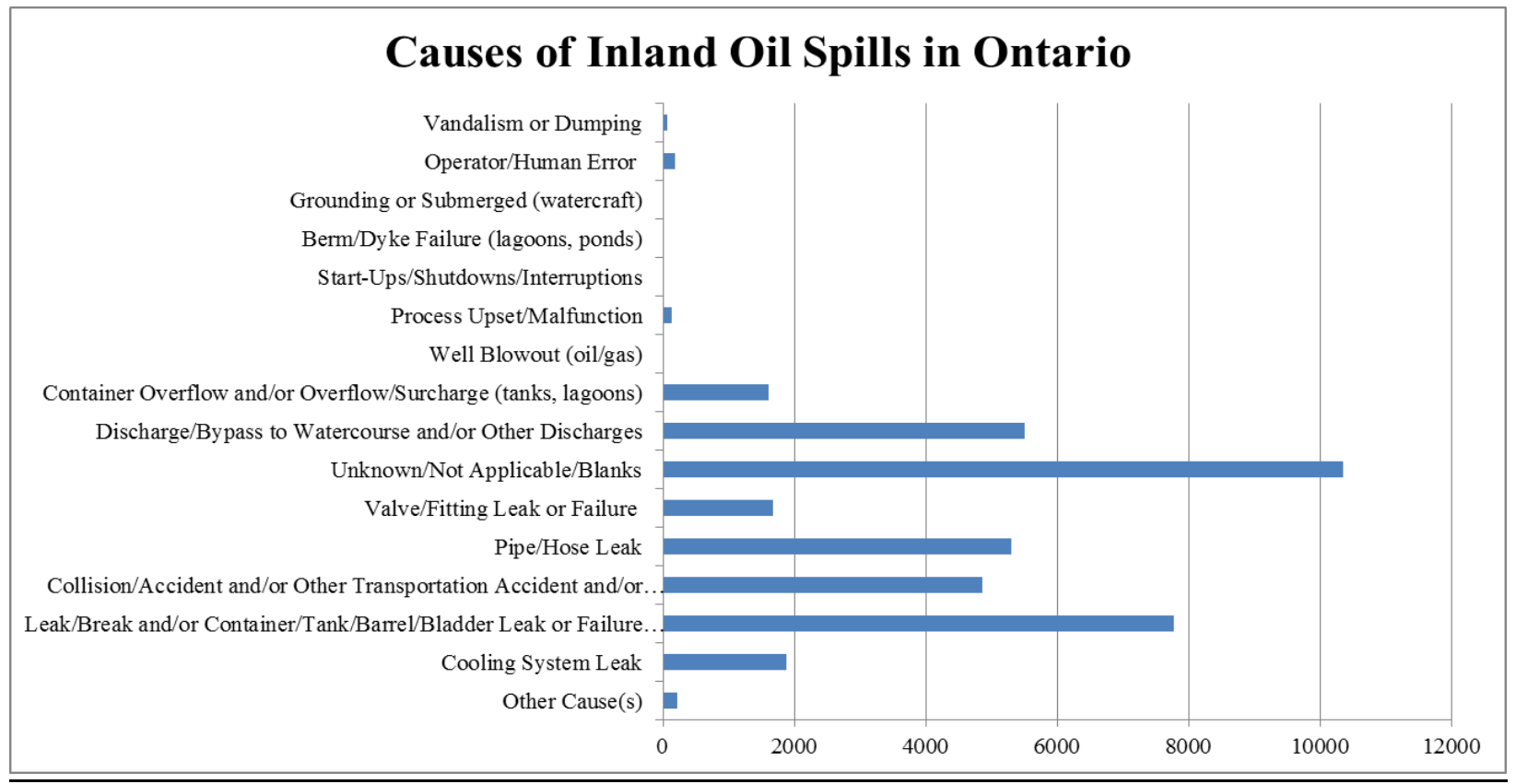

Figure 8. A bar chart of the causes of all inland oil spills based on preliminary analyses.

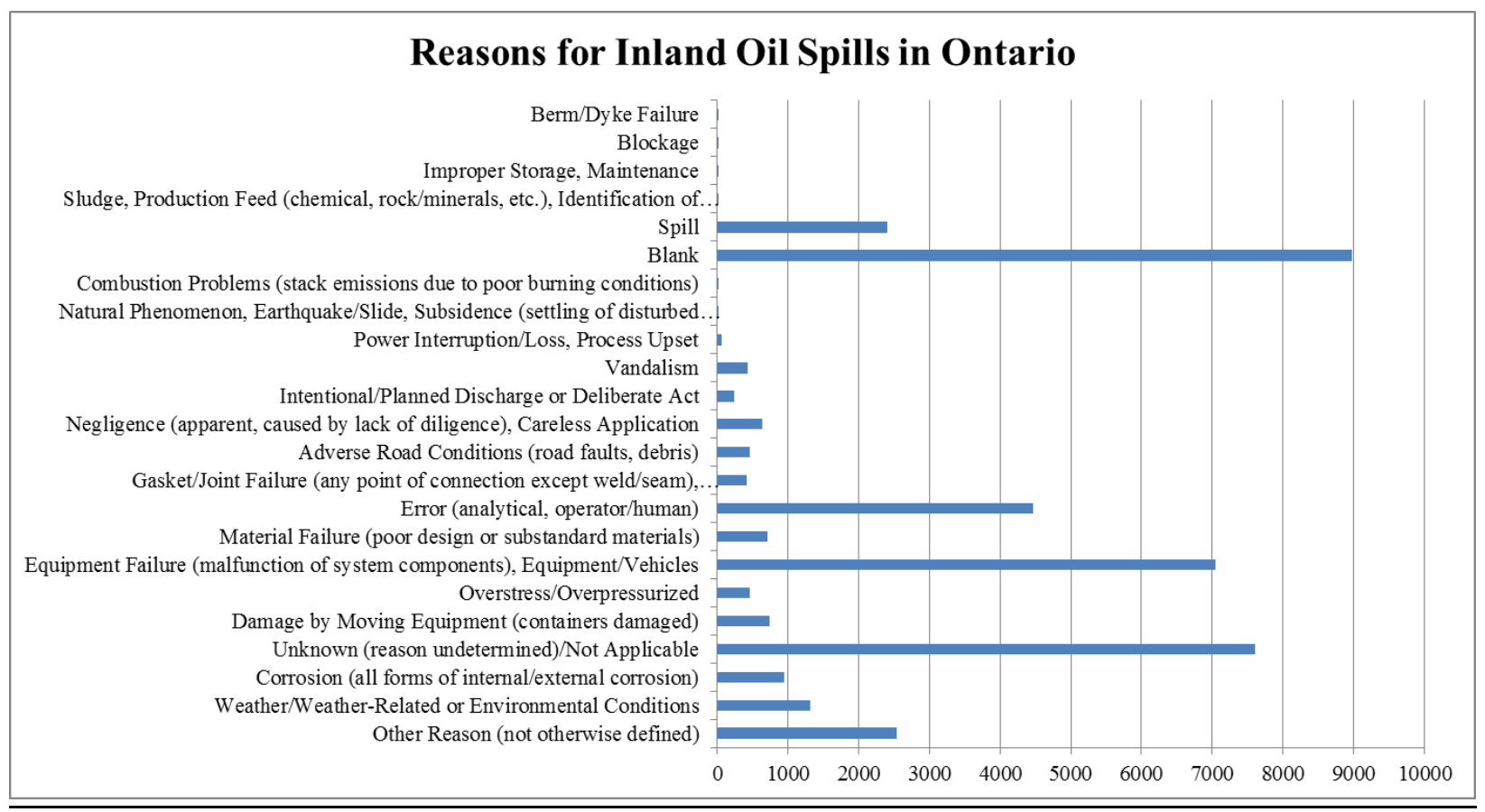

Figure 9. A bar chart of the reasons of all inland oil spills based on preliminary analyses. 
Figure 8 demonstrates that some of the general causes for all inland oil spill events are leaks or breaks of containment systems and pipe or hose leaks. Figure 9 demonstrates that some of the main reasons for all inland oil spill events are equipment failure or equipment/vehicular-related reasons and analytical or human error. The bar chart in Appendix B.1 demonstrates how soil and water environments are, in general, the environments which are most impacted by inland oil spills.

The given spill data for the earlier time period (1988-2007) only contained Longitude and Latitude values for spill records with site address data. This suggests that previous researchers geocoded spill locations based on site address to derive Longitude and Latitude values. In more recent spill data, Site UTM Easting and Site UTM Northing values were provided for a small fraction of site address data and with very few non-blank entries. For many spill records lacking detailed site addresses, zero values or blanks were mostly assigned as Longitude/Site UTM Easting and Latitude/Site UTM Northing values although some records without site addresses have geographic coordinates.

Therefore, the additional spatial data provided by geographic coordinates resulting from address geocoding does not compensate for the many limited or inaccurate spill site address data entries. It is recommended that Global Positioning System (GPS) devices or mobile GPS apps be used by the clean-up crew when attending the spill site. The caller reporting the spill may also be able to relay such information if asked. More specific and 
accurate address data is required to facilitate and improve spill management and spill model development.

The most recent spill data from 2007 to 2013 contain some GPS coordinates for spill records but most GPS coordinates are left unfilled or blank. GPS coordinates were available for almost every record in the reviewed (e.g. spill database for Alberta). This is ideal for spill database development because spatial or location-based data is important for planning tool development and spill data analysis. Ideally recorded spill characteristics which may prove useful for spill database analysis and model development are compared with currently recorded characteristics as listed in Table 5.

Table 5. Current vs. ideally recorded inland oil spill characteristics

\begin{tabular}{|l|l|}
\hline \multicolumn{1}{|c|}{$\begin{array}{c}\text { Currently Recorded Inland Oil Spill } \\
\text { Characteristics }\end{array}$} & \multicolumn{1}{c|}{$\begin{array}{c}\text { Ideally Recorded Inland Oil Spill } \\
\text { Characteristics }\end{array}$} \\
\hline $\begin{array}{l}\text { Some geographic coordinate data } \\
\text { (geographic coordinate system/UTM), site } \\
\text { address, municipality, region }\end{array}$ & GPS coordinates for all spills \\
\hline Incident date, date reported to SAC & Spill duration time \\
\hline Percentage of spill cleaned up & $\begin{array}{l}\text { Explanation for spills not cleaned up, } \\
\text { amount of oil recovered after each spill, } \\
\text { clean-up or remediation method used }\end{array}$ \\
\hline Environmental impact & Extent of damage caused by spill \\
\hline Nature of impact (soil, air, water) & The first medium impacted by the spill \\
\hline Brief incident summary & Meteorological and hydrological data \\
\hline
\end{tabular}

By collecting GPS coordinates for all spills, the efficiency of spill modelling will be enhanced by improving the accuracy of spill location data and alleviating the need for address geocoding. For this work, the current address or geographic coordinate data is assumed to be the starting point or point of origin of the spill. However, if this is not the case, an additional note should be made in a separate field. In general, more 
standardization and codification of spill characteristic entries is recommended with notes for exceptions and supplementary details placed in distinct and separate fields.

Meteorological data for each spill are proposed mainly for the purposes of distinguishing dry spills from wet spills. Dry spills occur during dry weather conditions and generally travel more slowly than wet spills which occur during precipitation events or are influenced by wet weather conditions. Wet spills may therefore require faster response times than dry spills and may prove to be more impactful and damaging to the environment.

For this research, it was assumed that all recorded and predicted spills are dry weather spills. Although climate data from external agencies and sources are readily available for the case study area to distinguish dry spills from wet spills, the only time variable in the data set is Interevent Time (days). Sub-daily data for the time of the spill event to coordinate the spill event and the rainfall event is not given. Moreover, rainfall events can be highly localized and may not occur in the exact same location as the spill event. The exact location of each spill event must therefore be coordinated with the corresponding rainfall event in order to classify the spill event as a wet event or a dry event. Meteorological and hydrological data are recommended for more detailed spill modelling because wind speed and air temperature are required to calculate oil evaporation (Farrar et al., 2014). 
Some of the ideal spill characteristics listed in Table 5, such as GPS coordinates for all spills, are recommended as an improvement to the existing database whereas others could be added to facilitate the development of spill management programs and policies. It is important to collect, record, and analyze spill data in an accurate and organized manner since spill data form the foundation for the development of a spill management framework.

The main spill database was first subdivided into three sub-databases (stationary, transportation-related and other) for each spill type based on the source of the spill. Source data indicates the origins of the spill in terms of the type of sector (e.g. electric power generation, petroleum refinery) or structure (e.g. pipeline, storage facility, plant), equipment (e.g. hose), and vehicle (e.g. motor vehicle, transport truck, watercraft). The first stationary spill sub-database contained a relatively low number of records, mainly as a result of excluding spill records with blank/unknown/not applicable/miscellaneous source entries as well as source entries listed as "other".

As a result, the main spill database was re-compiled and additional information from each spill record was used to determine the type of source for previously excluded data and to re-classify spill records. The development of the stationary spill sub-database concluded with 10,147 spill records. The result is that the majority of inland oil spills in Ontario can be classified as stationary (46\%), followed by transportation-related (34\%), and other spills (20\%), as illustrated in Figure 10. 


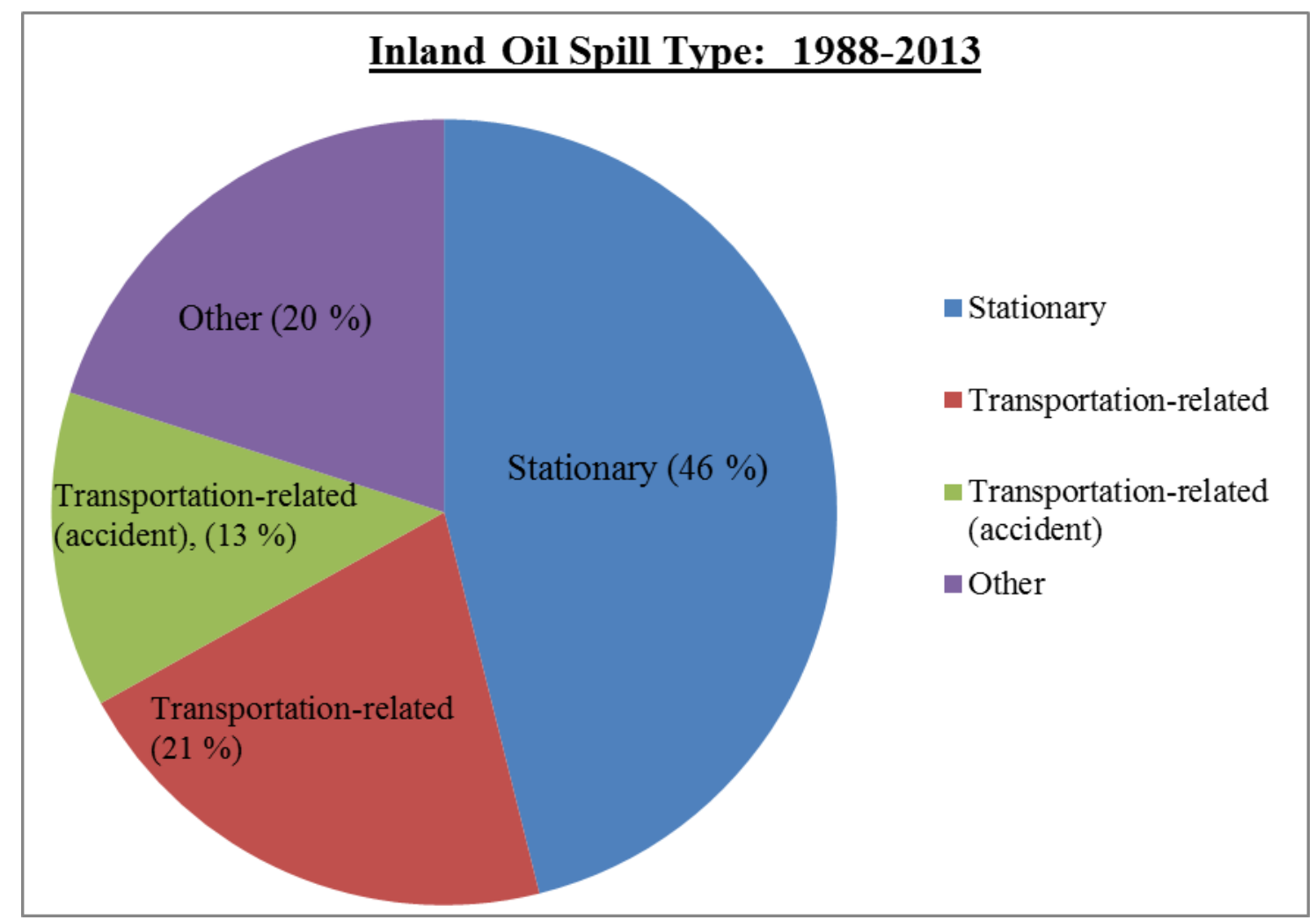

Figure 10. Type of inland oil spill as determined by the source of the spill.

Figure 10 illustrates that a sizable portion, $13 \%$, of transportation-related inland oil spills result from automotive accidents while $21 \%$ result from other transportation-related issues. Other transportation-related issues may include, for instance, mechanical failure of a large truck resulting in a significant amount of diesel leaking to the road while the truck is being driven.

As shown in Figure 10, collisions or accidents and other transportation accidents are one of the most common types of spill events based on the initial spill database containing all spill records. Equipment/vehicle malfunctioning is also one of the most common reasons to explain all inland spill events. These results initiated the investigation of transportation-related spills as a separate type of spill category. 


\subsection{Spatial Analysis of Transportation-Related Spills \& The Highway Spill Model}

The transportation-related sub-database was first geocoded using ArcGIS to produce a shapefile of 14,181 transportation-related spill points that have historically occurred within Ontario. Many of the addresses for transportation-related spill data are listed as major intersections or the nearest intersection to the spill occurrence because the exact address was unknown at the time and the call to report the spill was not being placed from a fixed location such as a building. Address data containing intersections were therefore used to identify transportation-related spills although the spill locations were usually not very accurate. The accuracy of the transportation-related spill data may have affected the spatial analyses performed by generating mapping clusters from hot spot analyses that are less accurate in terms of their representation of historically occurring transportation-related spills. Only historically occurring transportation-related spills were analyzed. Future transportation-related spill data were not predicted using the macro program as was carried out for stationary spills.

The Cause field in the spill database was re-categorized to reflect the refined focus of transportation-related spill analysis by classifying spill records as either road-related vehicle collisions (7,393 records) or vehicle-related incidents (6,491 records). After further development of the transportation-related spill sub-database, the resulting data set contains 13,884 spill records of inland oil spills occurring in relation to transport in Ontario. 
Spatial statistics were performed using an optimized version of hot spot analysis to identify statistically significant spatial clusters of high values or hot spots and low values or cold spots (ESRI, 2017). Figure 11 illustrates statistically significant hot and cold spots of inland transportation-related oil spills in Ontario from 1988-2013.

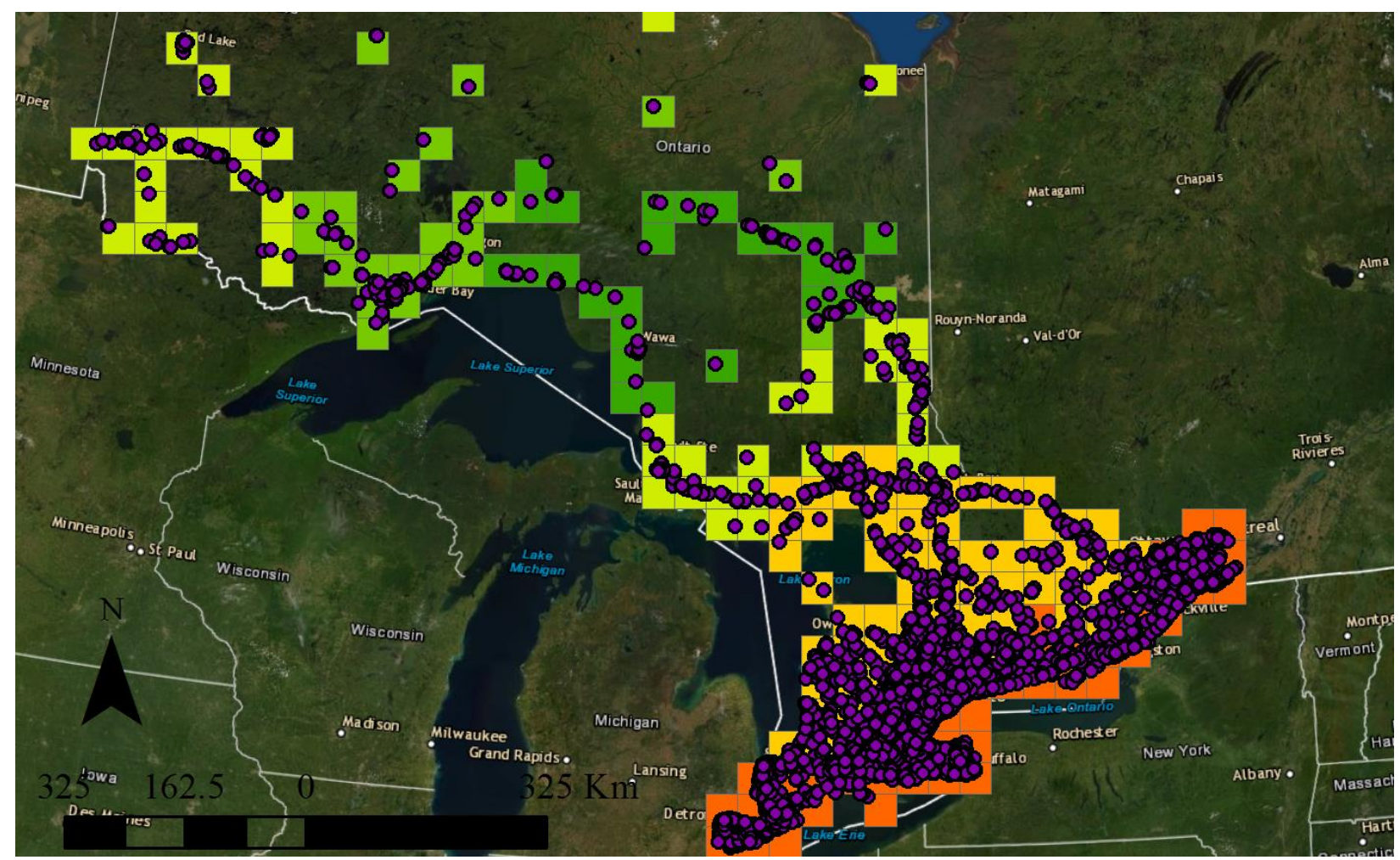

Figure 11. Statistically significant hot and cold spots of inland transportation-related oil spills in Ontario from 1988-2013.

The transportation-related spill points shown in purple in Figure 11 are overlaid on a fishnet polygon mesh which is situated on an ortho imagery layer of Ontario and surrounding regions. The underlying fishnet polygon mesh in Figure 11 is used to count the number of incident points within each polygon cell of the fishnet as part of the incident data aggregation method. Only cells with at least one incident point were used in the analysis (ESRI, 2017). 
The orange polygon cells in southern Ontario represent statistically significant hot spots which is consistent with the high concentration of spill points overlaying the fishnet polygon mesh in this region. The yellow-green areas in Figure 11 represent statistically significant cold spots, as illustrated by the relatively fewer amount of spill points on top of these cells.

Statistically significant hot spots of transportation-related inland oil spills are only located in southern Ontario, as demonstrated in Figure 11. This result can be explained by the predominance of population, industry, high traffic volumes and transport of goods in southern Ontario. Transportation-related spill policies and programs should therefore mainly target the highway and road system in the southern Ontario region.

The shapefile of transportation-related spill points displayed in Figure 11 represents incident data for the transportation-related spill spatial analysis. In this study, incident data represent all transportation-related spill events such as road-related collisions and vehicle-related incidents. The focus of optimized hot spot analysis for these events is on the presence or absence of the event (ESRI, 2017).

Therefore, the statistically significant hot or cold spots shown in Figure 11 are based on the presence or absence of the transportation-related spill event rather than a particular spill characteristic such as Spill Mass ( $\mathrm{kg}$ ) or Spill Volume (L) that is associated with each point. This enables a greater focus on the spatial aspects of the transportation-related spill occurrences in relation to the transportation system. 
Another incident data aggregation method was used to investigate the hot spot analysis of all transportation-related spills within Ontario for all oil types, as previously described. All model parameters remained the same except that a hexagon polygon mesh was used, instead of a fishnet polygon mesh, to count incident points within each polygon cell. Hexagons are suggested as preferable for certain analyses such as when you are considering a large area such as Ontario or including aspects of movement paths such as highways or roadways (ESRI, 2017). The results of employing a hexagon polygon mesh are shown in Figure 12. The transportation-related spill points are not displayed in Figure 12 to more clearly illustrate the colour variations of the hexagon polygon mesh.

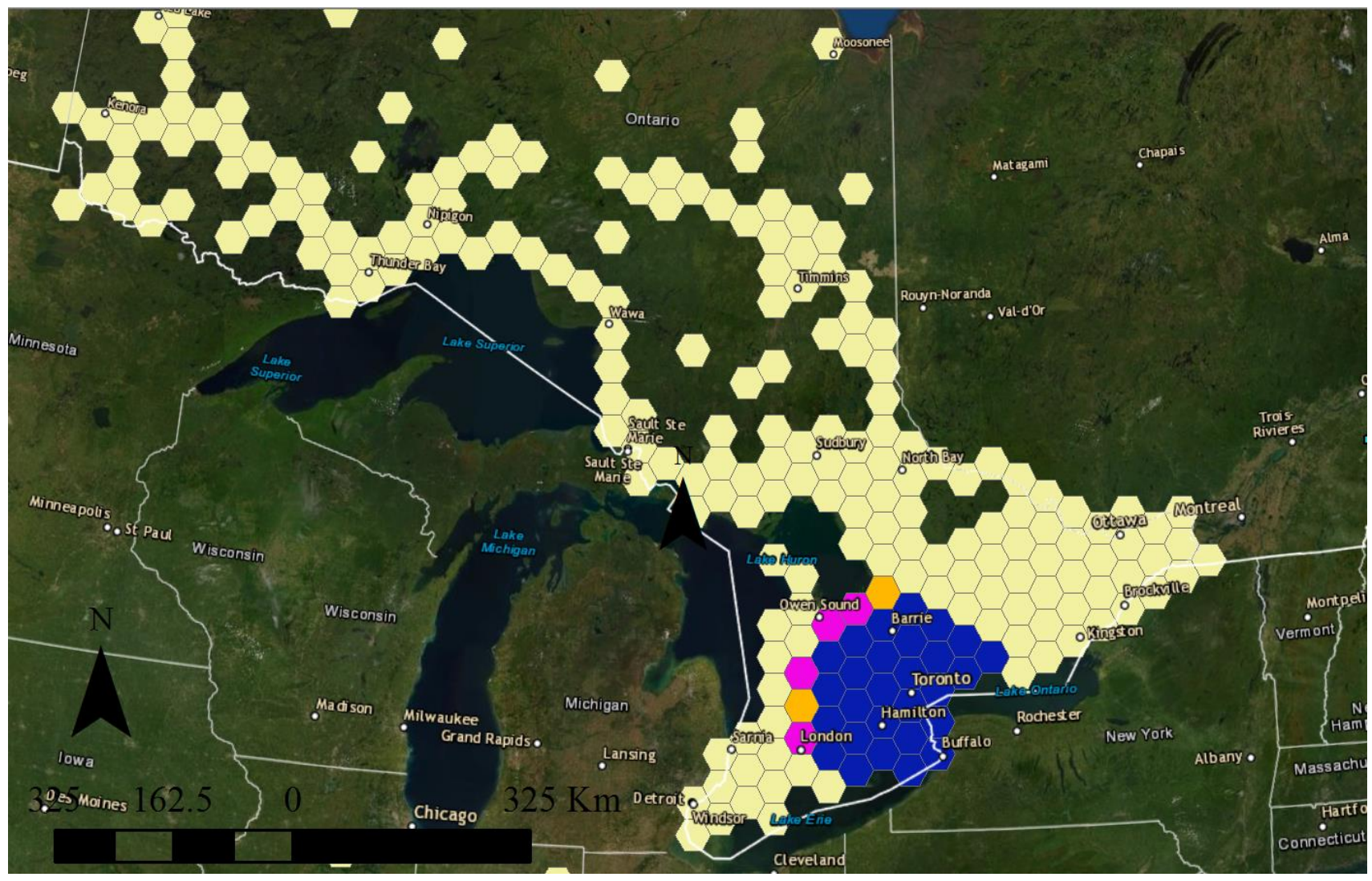

Figure 12. Optimized hot spot analysis using a hexagon polygon mesh for transportation-related inland oil spills in Ontario from 1988-2013. 
The beige areas in Figure 12 represent statistically significant cold spot areas while all other colours represent areas of statistically significant hot spots. There are fewer groups of statistically significant cold spots using this method as compared with the previous method using the fishnet polygon. However, the statistically significant cold spot areas comprise a greater portion of the province than the statistically significant hot spot area. The main statistically significant hot spot of varying intensities is visible in the central part of southern Ontario, encompassing both the Greater Toronto Area and the Golden Horseshoe region. A more specific result is provided by this method which can aid in focusing spill prevention strategies on certain areas with greater problems.

In Figure 11, the hot spot area in the southern area of the province is associated with the highest concentration of spill points. The results shown in Figure 12 provide additional value because the general hot spot area of the southern portion of the province has been narrowed down to the singular hot spot in the central part of the southern region. This result is not immediately obvious after overlaying the transportation-related spill points on the hexagon polygon mesh due to the high concentration of spill points scattered throughout the southern part of the province, as shown next in Figure 13. 


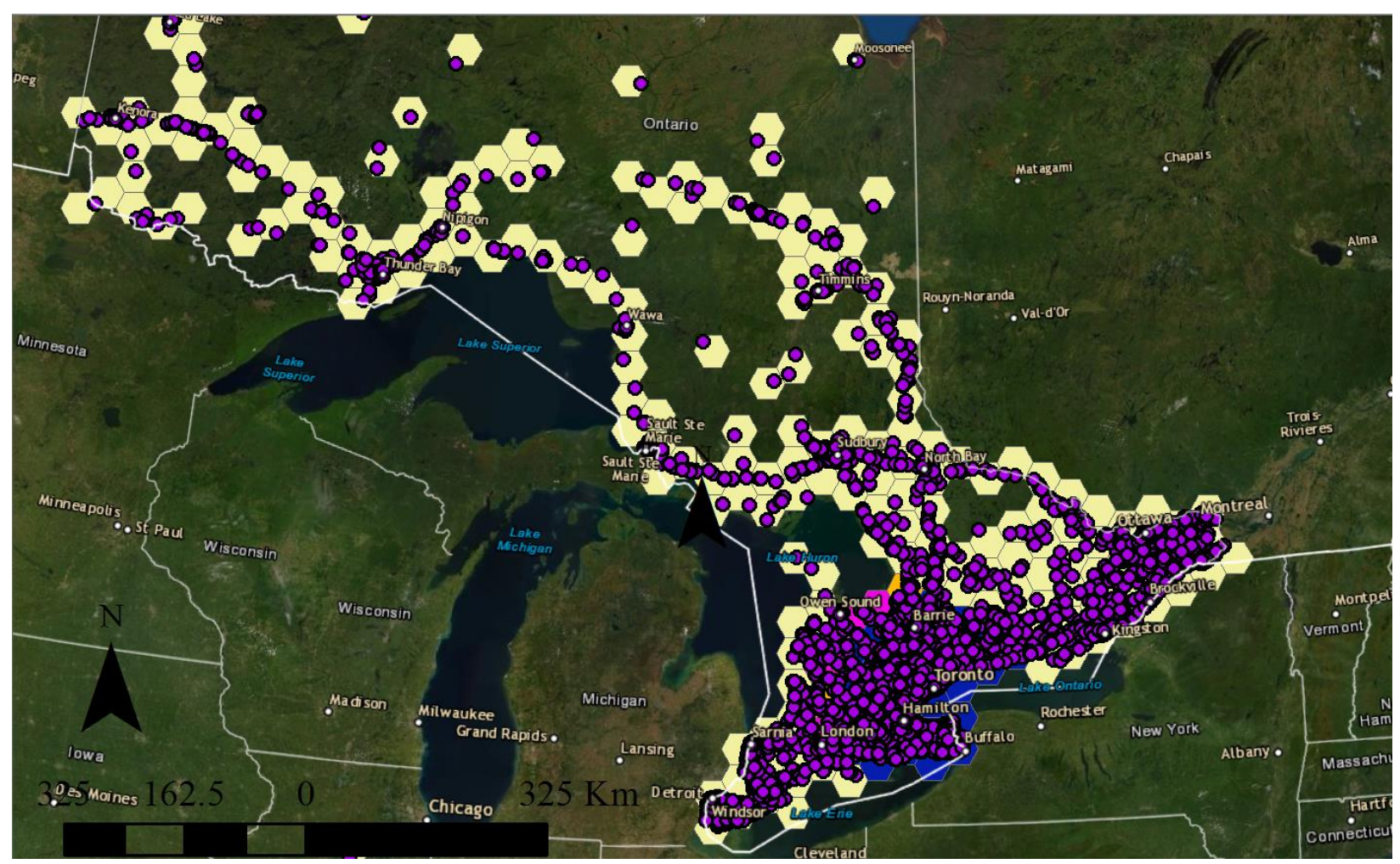

Figure 13. Spill points overlaid on the hexagon mesh of the optimized hot spot analysis.

The results in Figures 12 and 13 demonstrate that the main hot spot area of concern is the GTA and surrounding areas. Given that the Digital Elevation Model (DEM) is limited to the City of Toronto, Toronto was selected as the study area for the spatial analysis.

The number of road-related vehicle collisions which were extracted from the transportation-related spill sub-database is 7,393 and from among these, 866 records were extracted specifically for the City of Toronto. The resulting historical data set consists of 866 transportation-related spills which occurred in Toronto from 1988-2013. The 866 historical spill occurrences within Toronto were geocoded and mapped. Figure 14 illustrates that these spills appear to be fairly evenly distributed with almost no visible spatial clustering. 


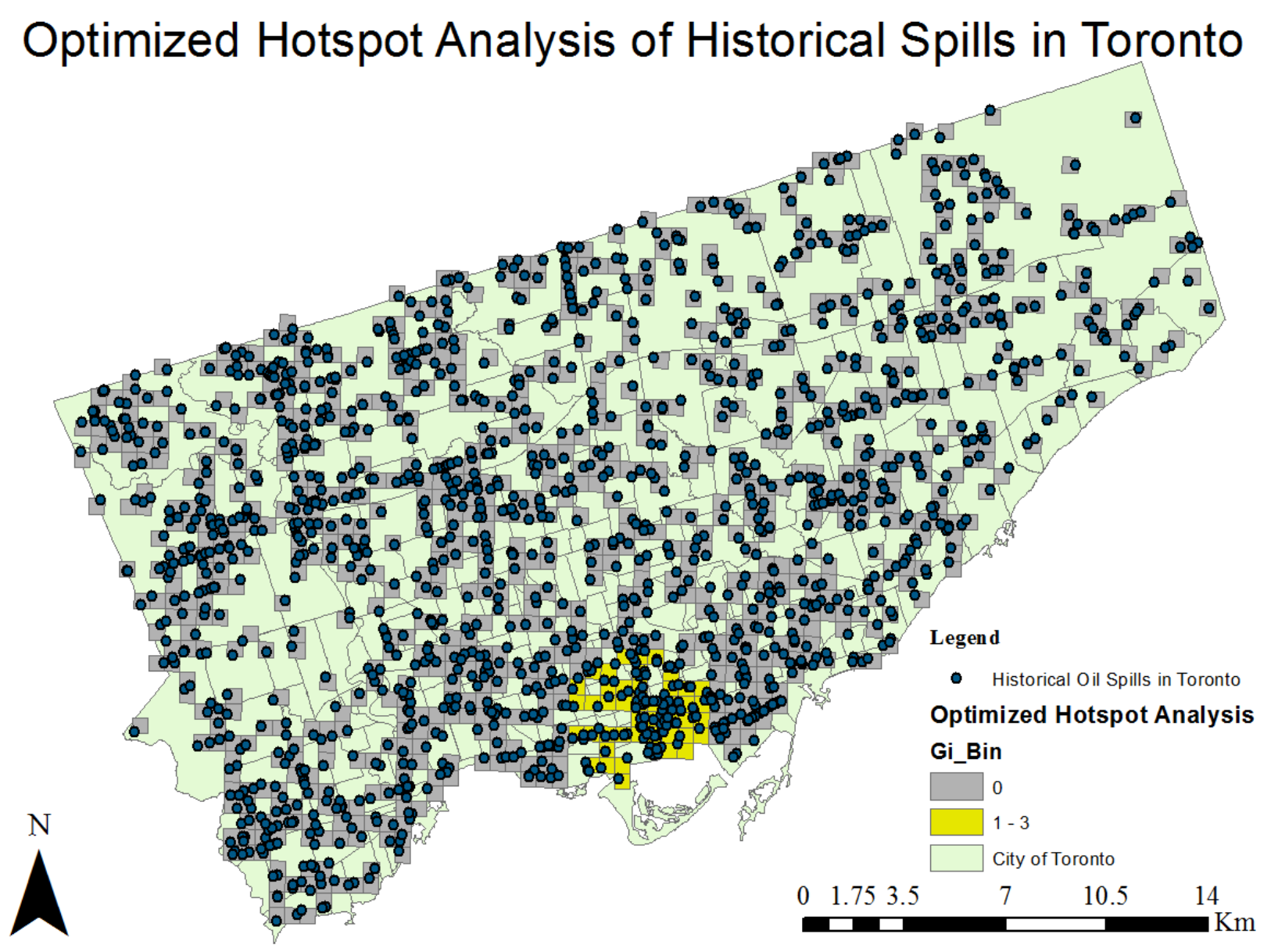

Figure 14. A map of a statistically significant hot spot beginning at the lakefront area in downtown Toronto.

The commonly used aggregation shape of fishnet polygons was used in an optimized hot spot analysis to identify statistically significant hot spots. One statistically significant hot spot area was found in the lakefront area of the downtown core in Toronto as shown in Figure 14. This result can be used to encourage a heightened awareness of spill response and clean-up efforts in this area. The variety of street or road types contained in the hot spot shown in Figure 14 made it difficult to detect a discernible pattern or relationship between spill events and road type. 
There was no apparent overall effect from experimenting with other tool parameters such as changing the grid cell size or providing a census tract bounding polygon. For example, by selecting the option to apply a census tract bounding polygon, the empty green areas in the map of Toronto in Figure 14 were included in the analysis. However, since there were no underlying incidents in those areas the fishnet cells received an incident count of zero. Therefore, the inclusion or exclusion of the empty green areas resulted in an incident count of zero. Since no input features overlay these areas, a resulting p-value and z-score were not provided.

The optimized hot spot analyses tool was applied to the set of spill points derived from the initial transportation-related sub-database as well to the points derived from the subsequent data set of 866 transportation-related spills of road-related vehicle collisions that occurred in Toronto. Both sets of spill points were representative of all oil types. Based on the results, recommendations were made for spill management strategies. Organizations can also follow the same approach to develop their own spill management strategies.

To investigate a specific road type, spills within 50 metres of major Toronto highways were selected and extracted from the spill points shapefile shown in Figure 14. These selected spills are displayed in Figure 15, with the majority of spills occurring on, at or near interchanges, including a few ramps. A total of 178 transportation-related spills were selected and approximately $75 \%$ occurred at interchanges, $8 \%$ occurred on the highway, and $17 \%$ occurred at unspecified locations. 


\section{Historical Oil Spills Within 50 m of Major Toronto Highways}

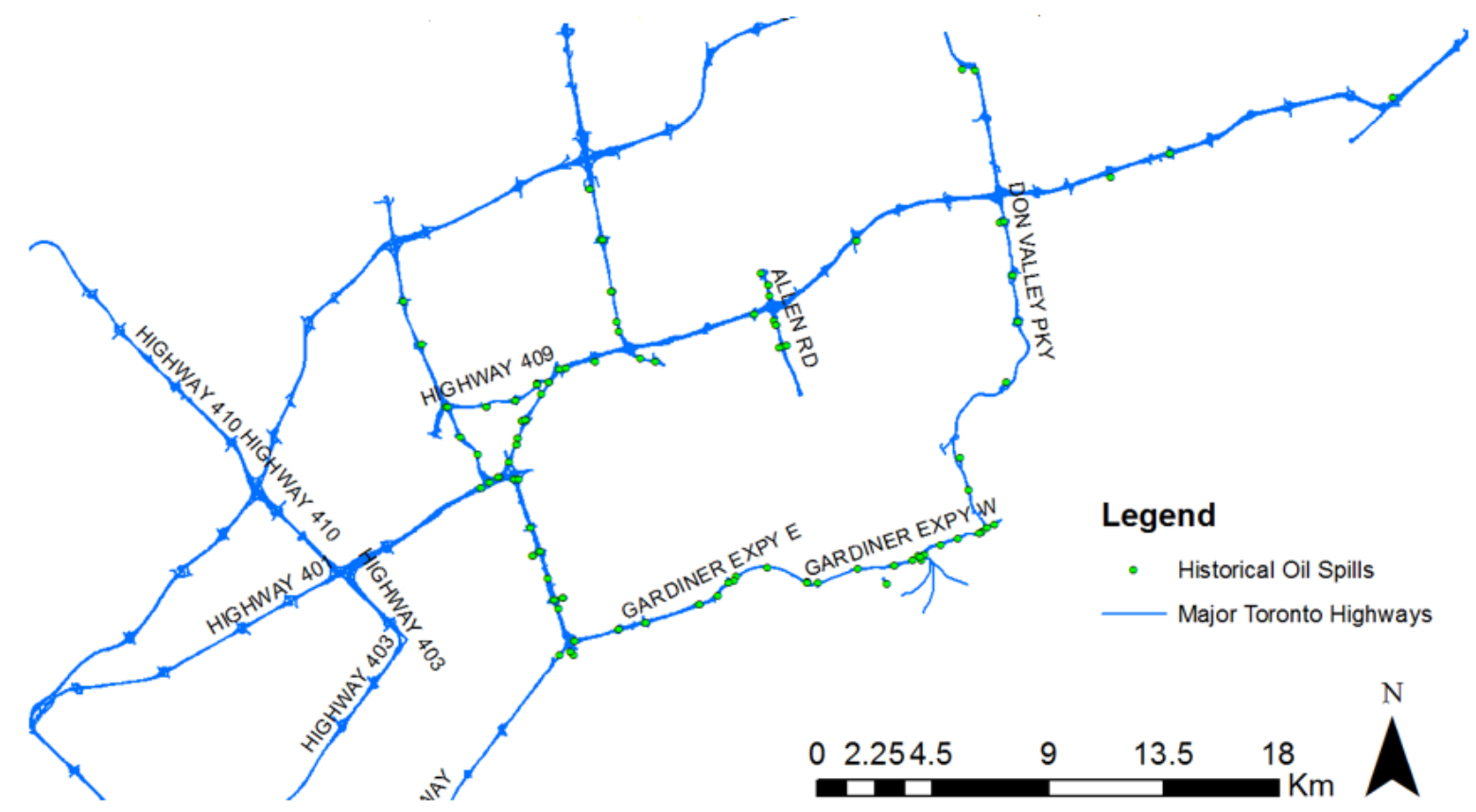

Figure 15. A map of spills within 50 metres of major Toronto highways, which often occurred at interchanges.

These results indicate that the majority of highway-related spills involve interchanges which suggests that the geometric design parameters or characteristics of interchanges could be improved to prevent or minimize future spill occurrences. Since the exact point of transportation-related spill occurrences has only been estimated using intersection names, mile markers and other inexact measures, more accurate spill point data is required to pinpoint which highway characteristic should be modified to prevent future highway-related spill occurrences. Historical data of road-related collisions in Toronto consists of $20.55 \%$ highway spills and $79.45 \%$ non-highway or local road spills. Therefore, future spatial analyses should also investigate non-highway or local road spills. 


\subsection{A Simple Prototype of a Web-Based GIS System for Inland Oil Spill Data in Ontario}

In earlier stages of research, after a single-machine deployment was configured for ArcGIS for Server 10.1, four basic interactive map services were published through ArcMap 10.1 and made available online via ArcGIS Server Manager. Locations of either stationary or transportation-related spills in Ontario municipalities were two basic map services that were developed. An illustration of the map service for transportation-related land-based oil spills in Ontario is given in Figure 16.

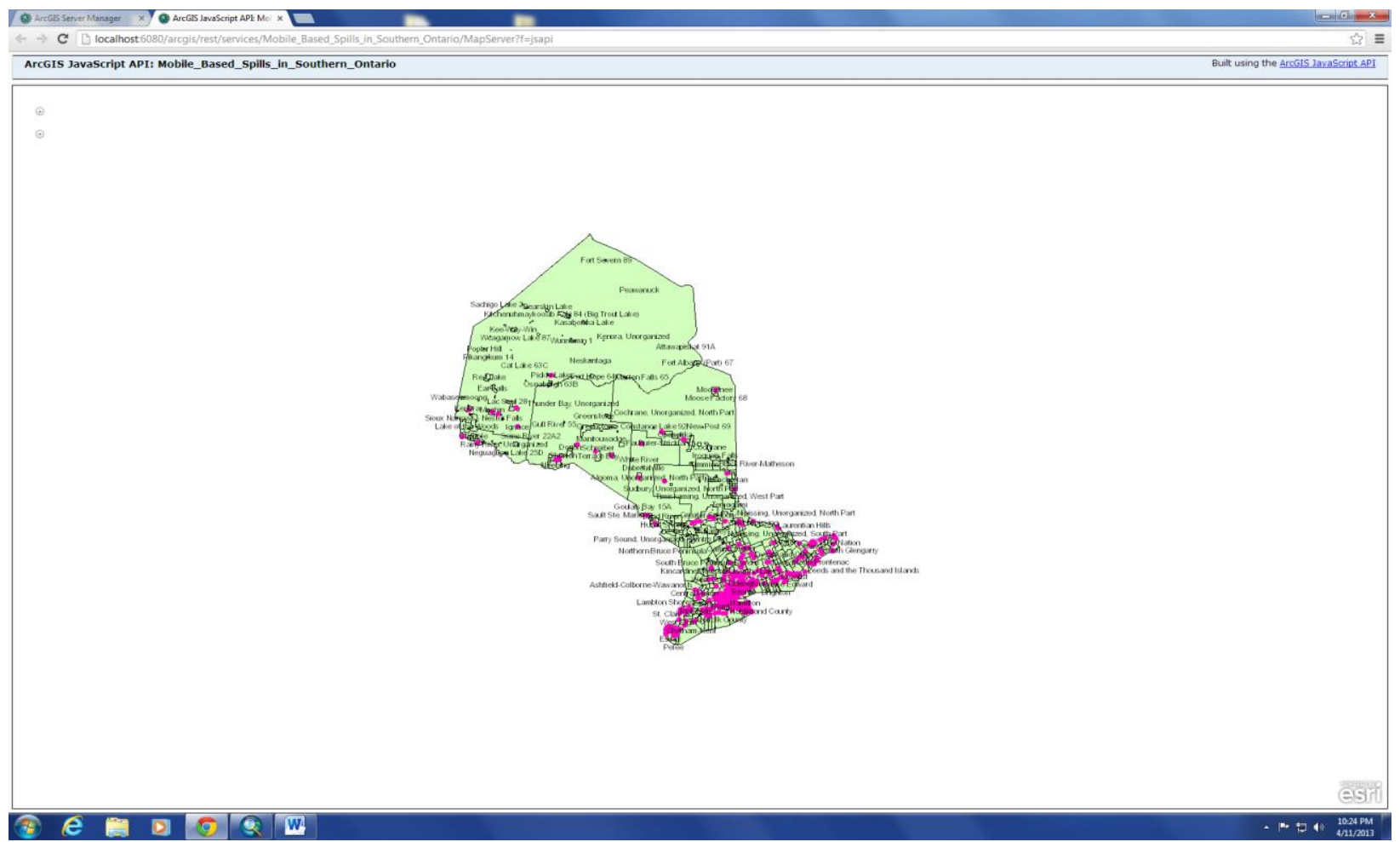

Figure 16. Map service illustrating transportation-related land-based oil spills in Ontario.

Although a small sample of transportation-related spill data was used, the results in Figure 16 demonstrate that the highest concentration of spills occurred in southern Ontario which is the same result derived from the initial trials of the hot spot analysis. 
The map service allows an individual to see the locations of highly concentrated spills which can then be verified as hot spots using the optimized version of hot spot analysis available in ArcGIS. Information pertaining to each spill such as the address and city can be obtained by clicking on the spill point as shown in Figure 17.

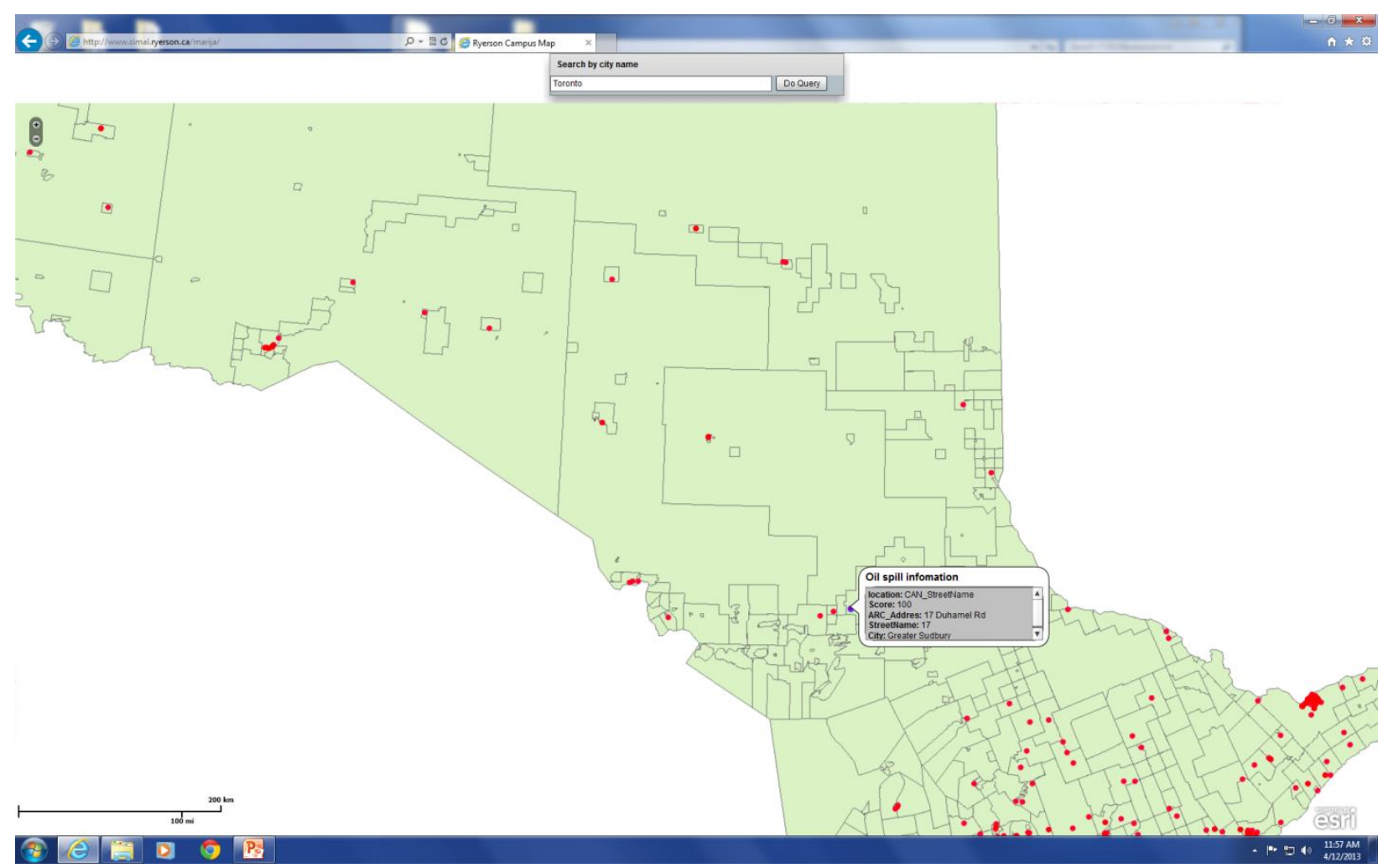

Figure 17. The retrieval of spill information by selecting spill points on the map service.

The other two map services developed showcase these spill locations as well as additional information such as hydrography (rivers, lakes, and creeks) and major roads and highways. An example of the map service with other data is shown in Appendix C.1.

\subsection{Statistical Analysis of Main Spill Database \& Stationary Spill $\underline{\text { Sub-Database Results }}$}

The development of the main spill database and corresponding sub-databases was a somewhat cyclical and evolutionary process. Stationary spills are defined as on-land oil 
spills occurring at fixed locations in which the source of the spill does not originate with a moving vehicle or with the contents being transported by a moving vehicle. Therefore, spills occurring at gas stations due to faulty nozzles or improper filling of the tank or vehicle are considered stationary spills and were included in the data set.

After the initial main spill database was re-compiled, the stationary spill sub-database contained 11,282 spill records. The primary spill characteristics subject to analysis were three numeric variables (Spill Mass (kg), \% Clean-Up, Interevent Time (days)) and five non-numeric variables (Oil Type, Sector/Source Type, Client Type, Cause, Reason). Client type represents the domain or field in which the spill occurred (business/corporation, government, residential/individual, other/multiple client types, and unknown/uncertain/N/A). Cause explains what happened (container or tank leak/failure/overflow, discharge/dumping, pipe/hose/valve/fitting leak or failure, spill) and reason explains why it happened (equipment or material damage/failure, intentional/planned/vandalism, negligence/operator error, weather/environmental conditions, other, unknown/uncertain/N/A). Low values of statistically insignificant correlation were found between the following variables: Sector/Source Type and Client Type (0.33); Sector/Source Type and Cause (0.26); and Cause and Reason (0.29).

A multiple linear regression was performed using statistical software with each of the three numeric variables as the y-variable and the remaining numeric and non-numeric variables as the $\mathrm{x}$-variables. There is no evidence for a strong linear relationship in any of the three cases since the $\mathrm{R}^{2}$ value is close to zero. The observed residuals do not lie on 
the straight line of the normal probability plot of residuals, implying that they are not normally distributed. In the case of the y-variable as \% Clean-Up, the observed residual values form an S-shaped pattern around the line, as shown in Figure 18, implying that the y-variable requires transformation (StatSoft, 2017).

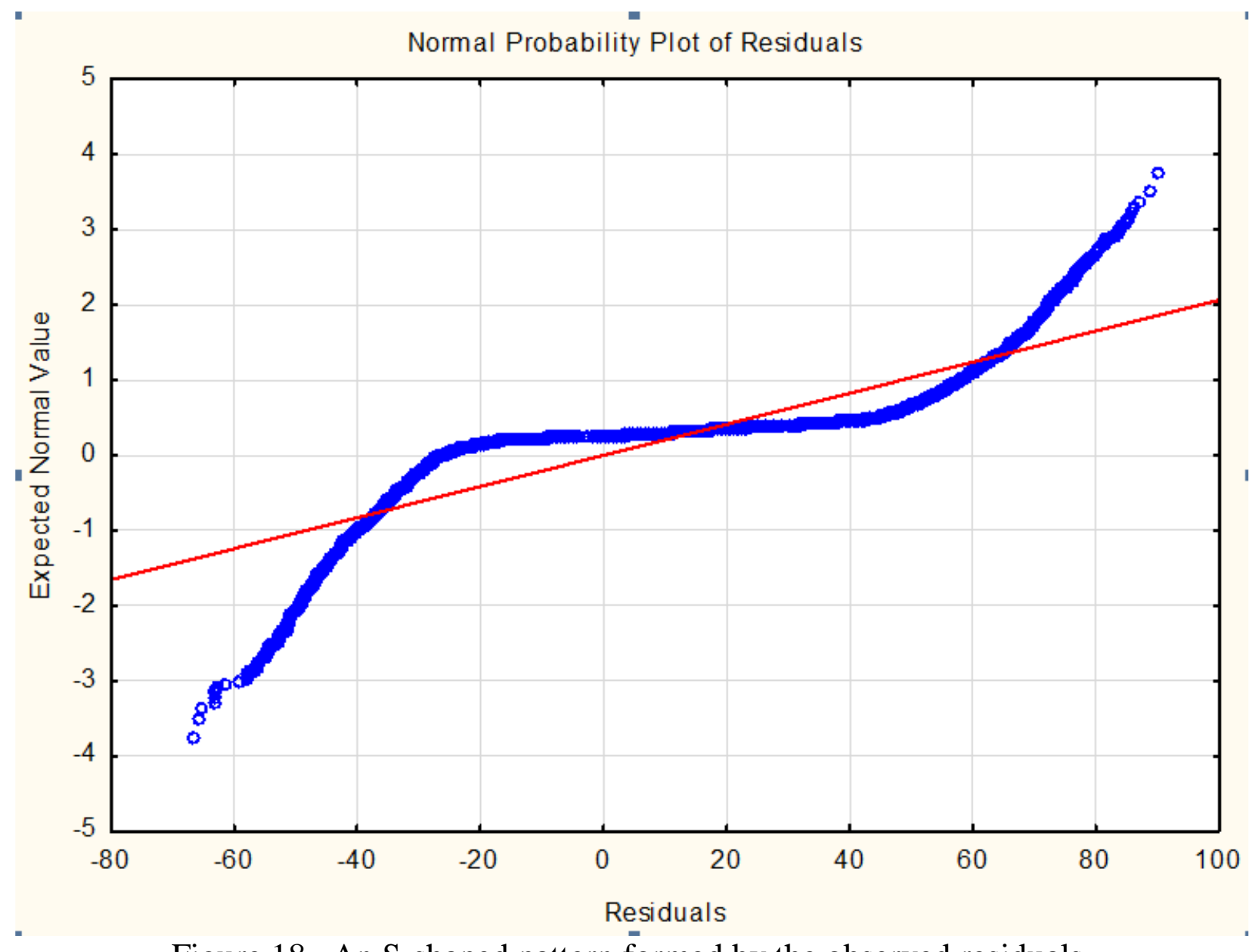

Figure 18. An S-shaped pattern formed by the observed residuals.

After using the $\log$ transformation to investigate relationships between the numeric variables, no significant linear regression results were found.

For the overall stationary spill database, regression relationships between the variables are essentially non-existent. Regression analyses were then performed for the most common type of oil spilled, light petroleum oils (e.g. diesel, furnace oils, mineral oils). 
The number of oil spills involving light petroleum oils from 1988 to 2013 in the initial stationary spill data set is 4,581 as shown in Figure 19 which categorizes oils according to SAC Material/Contaminant Code category names.

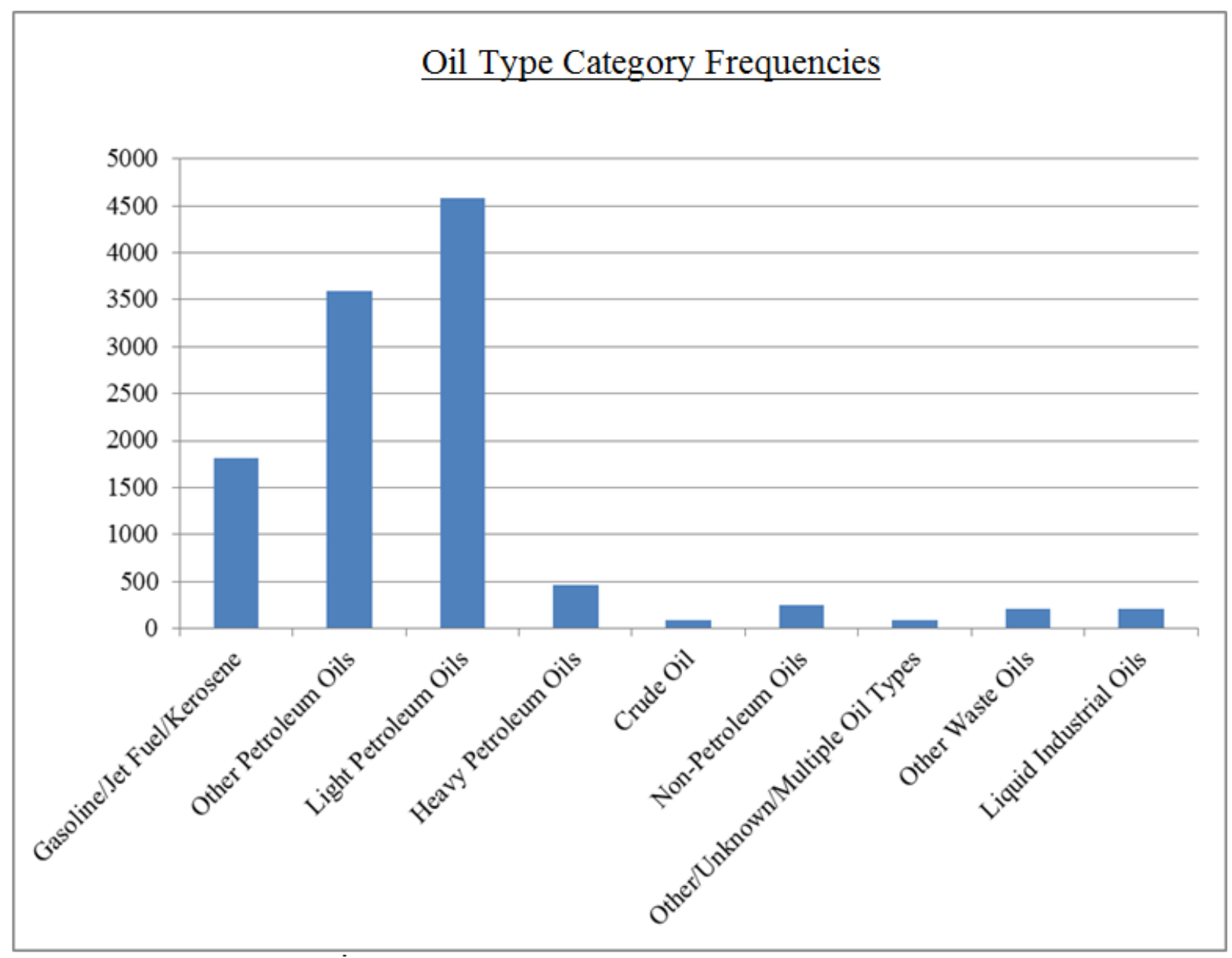

Figure 19. Histogram of SAC oil type categories.

For regression analysis of light petroleum oils, there was one less non-numeric x-variable since oil type remained constant. Multiple linear regression was performed for all three numeric variables as y-variables using a stepwise forward method as before, however no significant relationships were found. The only correlation values found were low, 
statistically insignificant correlation values between the variables Sector/Source Type and Cause (0.21).

Since the regression results did not demonstrate any statistically significant relationships between the eight variables involved, a Principal Components and Classification Analysis (PCA) was used as a multivariate exploratory technique. For the first PCA experiment, the three main numeric variables (Spill Mass (kg), Interevent Time (days), \% Clean-Up) were included with nine text variables (Nature of Impact, Reason, Cause, Client Type, NAICS Sector, Source Type, Detailed Oil Type, Final Municipality/Site Municipality, Final Region/Site District Office). All variables were considered as active and not supplementary variables to the analysis.

An initial result is a table of the eigenvalues of the correlation matrix and related statistics in which each factor corresponds to an eigenvalue. The eigenvalues are arranged in decreasing order to indicate the significance of respective factors in explaining the variation of the data (StatSoft, 2017). To determine which factors to retain for interpretation, the simplest criteria are to use as many factors as the number of eigenvalues that are greater than one. In this experiment, the first five factors are greater than one yet account for only approximately $58 \%$ of the total variation.

Another method to determine which factors to retain is to analyze the resulting scree plot as shown in the next figure, Figure 20. A scree plot is a graph illustrating successive eigenvalues as a simple line plot. It has been suggested to find the point on the plot 
where the smooth decrease of eigenvalues appears to level off to the right of the point and select no more than the number of factors to the left of this point. Points after eigenvalue number 5 in Figure 20 begin to level off which agrees with the previous result of taking the first 5 factors greater than one. However, if one applies the rule literally than no more than 4 factors should be selected as there are 4 points to the left of eigenvalue number 5 , which is the fifth point at which the decline in eigenvalues begins to level off.

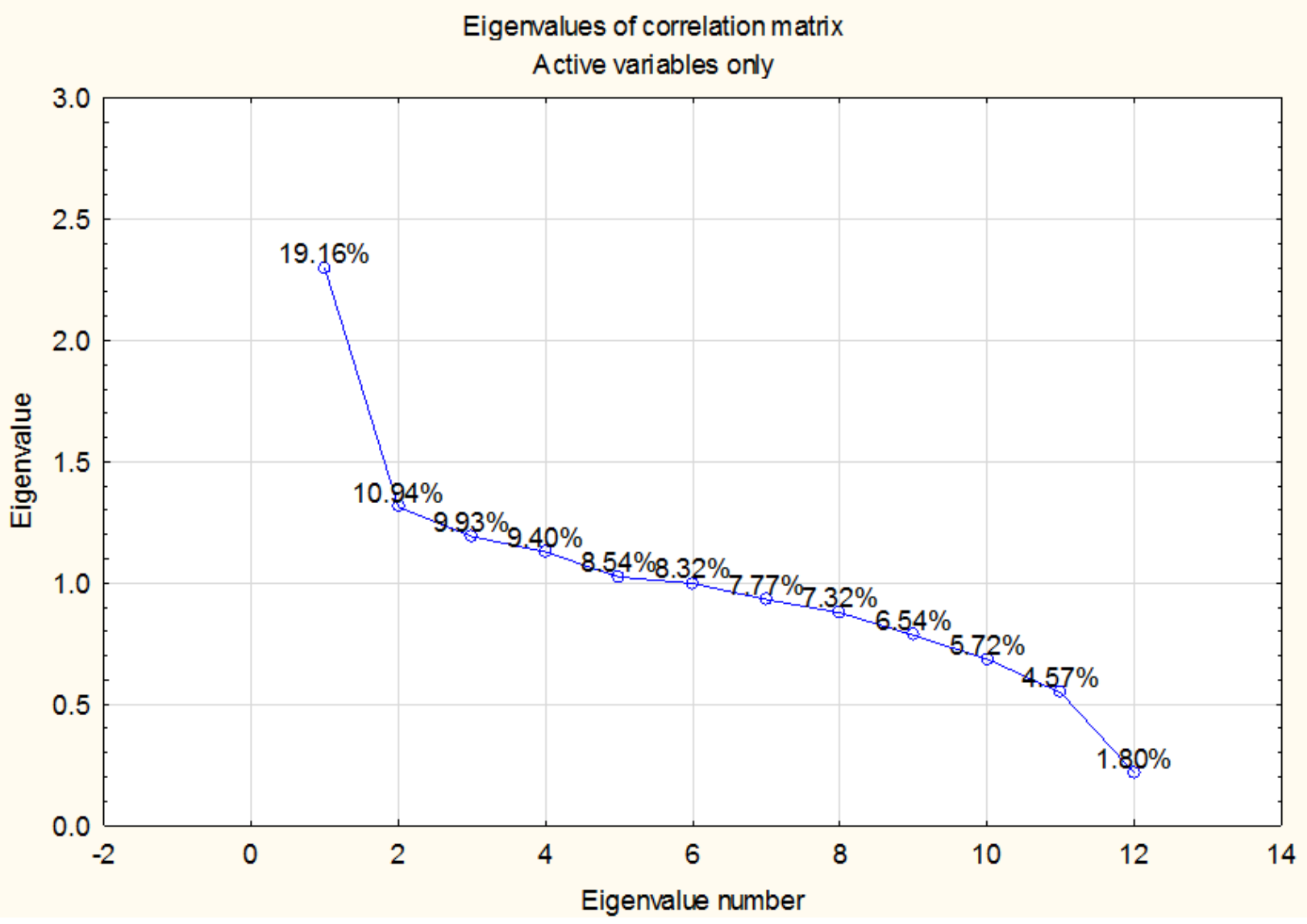

Figure 20. Scree plot of first PCA of initial stationary spill sub-database.

Lastly, the plot of factor coordinates facilitates the interpretation of factors by displaying the factor coordinates for two factors. The two factors plotted in the plot of factor coordinates of Figure 21 are the first two factors which only account for approximately 
$30 \%$ of total variation. The plot shows a unit circle in which all factor coordinates must fall within the unit circle. The unit circle provides a visual indication or scale as to how well each variable is represented by the current set of factors. The closer a variable is to the unit circle, the better is its representation by the current set of factors (StatSoft, 2017).

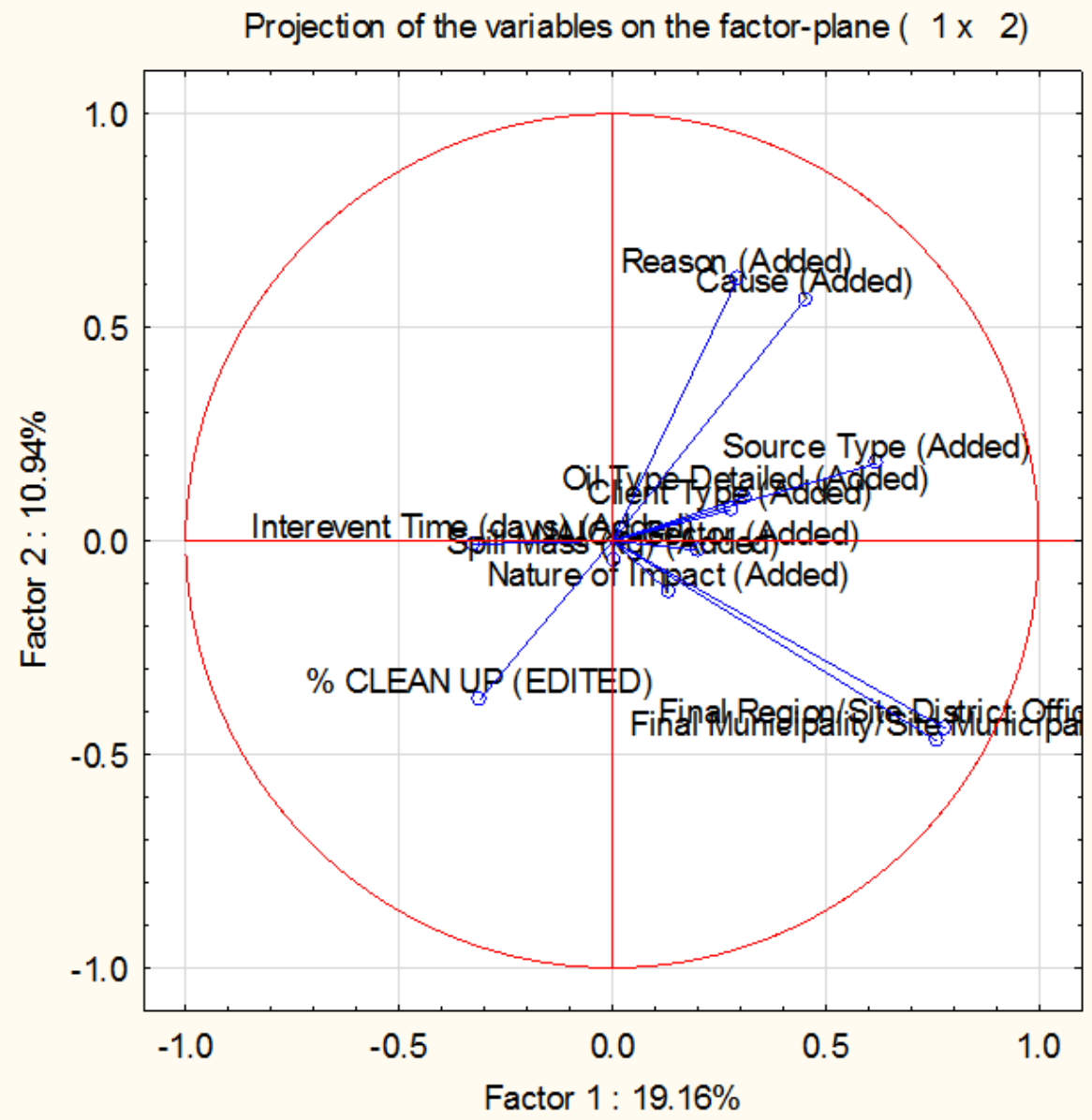

- Active

Figure 21. The plot of factor coordinates for the first PCA experiment with all variables as active variables.

Figure 21 demonstrates how most variables are centered within the plot and do not lie close to the unit circle. The variable names that overlap at the origin and in other places, illustrate the closeness of the factor coordinates in the factor-plane. Variables that are close to the unit circle are Reason, Cause, Final Region/Site District Office, Final 
Municipality/Site Municipality Office and \% Clean-Up. As a result, they are better represented by the current set of factors. The overall quality of representation is $81.40 \%$ when using eight factors. The first five factors have eigenvalues greater than one whereas the remaining three factors have eigenvalues slightly less than one but can still incorporated to represent and evaluate the quality of representation.

The mathematical procedure known as Statistica PCA was used to represent the set of multivariate variables, with possible correlations, by a smaller number of uncorrelated variables, the principal components, by using the NIPALS algorithm. Statistica PCA is a PCA technique specific to the Statistica software program. It can implement PCA using the state-of-the-art algorithm known as Nonlinear Iterative Partial Least Squares (NIPALS) in a mathematical procedure known as Statistica PCA. The method is particularly well-suited when a large number of predictor variables are involved (StatSoft, 2017).

The three main numeric variables were always selected in combination with text variables which ranged in number from one or two to fourteen when performing various trials and experiments. Regardless how few or how many qualitative variables were used in conjunction with the quantitative variables, nine or ten principal components were extracted to explain approximately $58 \%-61 \%$ of the sum of squares. Smaller sets of variables sometimes resulted in faster iteration run times which can be helpful since the iterative process of PCA techniques is sometimes quite lengthy. 
Another consistent and key result from the varying PCA experiments is the determination of the order of variable importance. The three numeric variables were always listed or shown as the most important, followed by all text or categorical variables. The order of importance of the three numeric variables is: (1) Spill Mass (kg), (2) Interevent Time (days), and (3) \% Clean-Up. The Nature of Impact and Client Type categorical variables followed the three numeric variables although the exact category varied since each category of each variable was treated separately in the resulting output lists and graphs. Block variables were used to assign categories of the same categorical variable to the same block number to determine, more generally, the ranking of variable importance. Despite this effort, each category of each categorical variable was still treated and listed separately.

A scatterplot of the loading factors between the first two principal components, called a loading scatterplot, was used to analyze the relation between the variables and identify the most influential ones in determining the PCA model. The scatterplot in Figure 22 illustrates a high degree of clustering in the centre by all variables except variable numbers 5,25 , and 51 which are the continuous or non-categorical variables. 


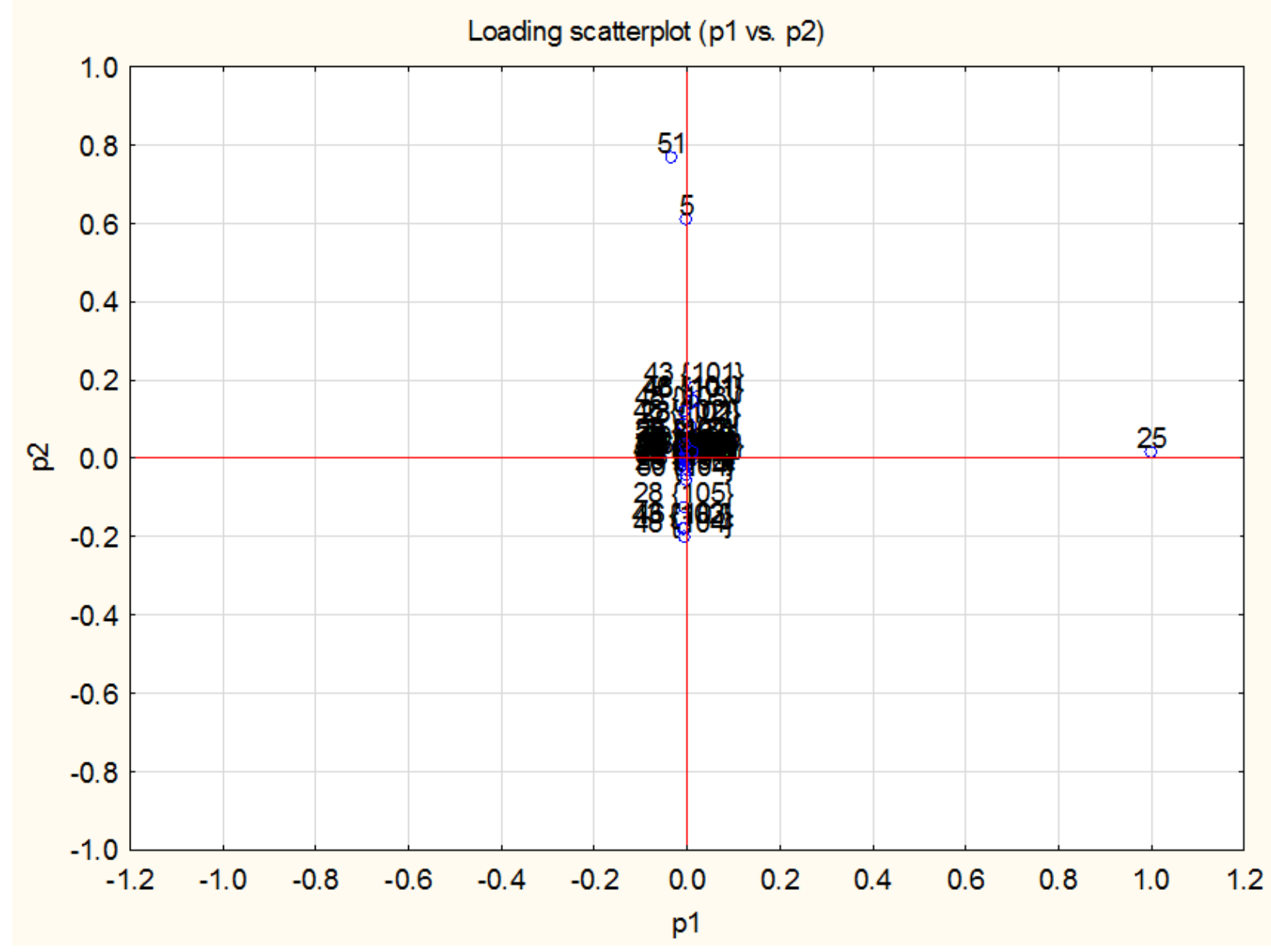

Figure 22. Loading scatterplot of component 1 and component 2.

Variables that are placed close to each other in the loading scatterplot influence the PCA model in similar ways which also denotes that they are correlated. Since all the non-numerical variables are clustered, they influence the model in similar ways. One more categorical variable or category of a categorical variable does not add a new kind of influence to the PCA model. Also, the further away a variable is from the origin, the more influential the variable is in determining the PCA model. All non-numerical variables are clustered around the origin while the three numeric variables are quite 
distant from it. This further implies that the three main numeric variables are much more influential than the group of non-numeric variables.

According to Figure 22, Spill Mass (kg) (variable 25) is the most influential, followed by $\%$ Clean-Up (variable 51) and IET (days) (variable 5). This differs slightly from the order of variable importance listed as Spill Mass (kg), IET (days) and \% Clean-Up. Therefore, the importance and influence of a certain variable are not entirely the same according to the resultant output of PCA.

Lastly, the results of the data mining approach on the subset of 2,330 stationary spills were unexpected as the output measure of error rate (\%) on the testing sample was not given, however the residual (mean sum of square) was given for both the testing and training samples. The residual is the difference between the predicted value of the target variable (Spill Mass ( $\mathrm{kg}$ ) in this study) and the actual value. The neural network model had the highest residuals for both the testing and training samples indicating that it is the least accurate of the three at predicting the target variable for this data set. The case-by-case deployment results varied as each model more closely predicted target variable values more closely at times than other models.

\section{5 $\underline{\text { Simulation Data Analysis \& Macro Program Results }}$}

\subsubsection{Input Simulation Data Analysis}

Stationary spills of gasoline in the Humber Watershed were selected as the input data set to form the basis of simulation studies. Figure 19 illustrates that gasoline is the third 
most commonly spilled oil within the stationary spill sub-database. The density of gasoline is $0.72 \mathrm{~g} / \mathrm{mL}$ (at $15^{\circ} \mathrm{C}$ ) (Fingas et al., 2011) and the density of water is approximately $1 \mathrm{~g} / \mathrm{mL}$ at room temperature. Both substances also have relatively low viscosities and are considered to be fairly "light" liquids that flow in a similar manner when spilled on the ground. Mock exercises of gasoline spills in paved areas can be performed using water as a substitute. Gasoline is also commonly spilled at fixed locations such as service stations and factories.

\section{Gasoline Spills in the Humber River Watershed}

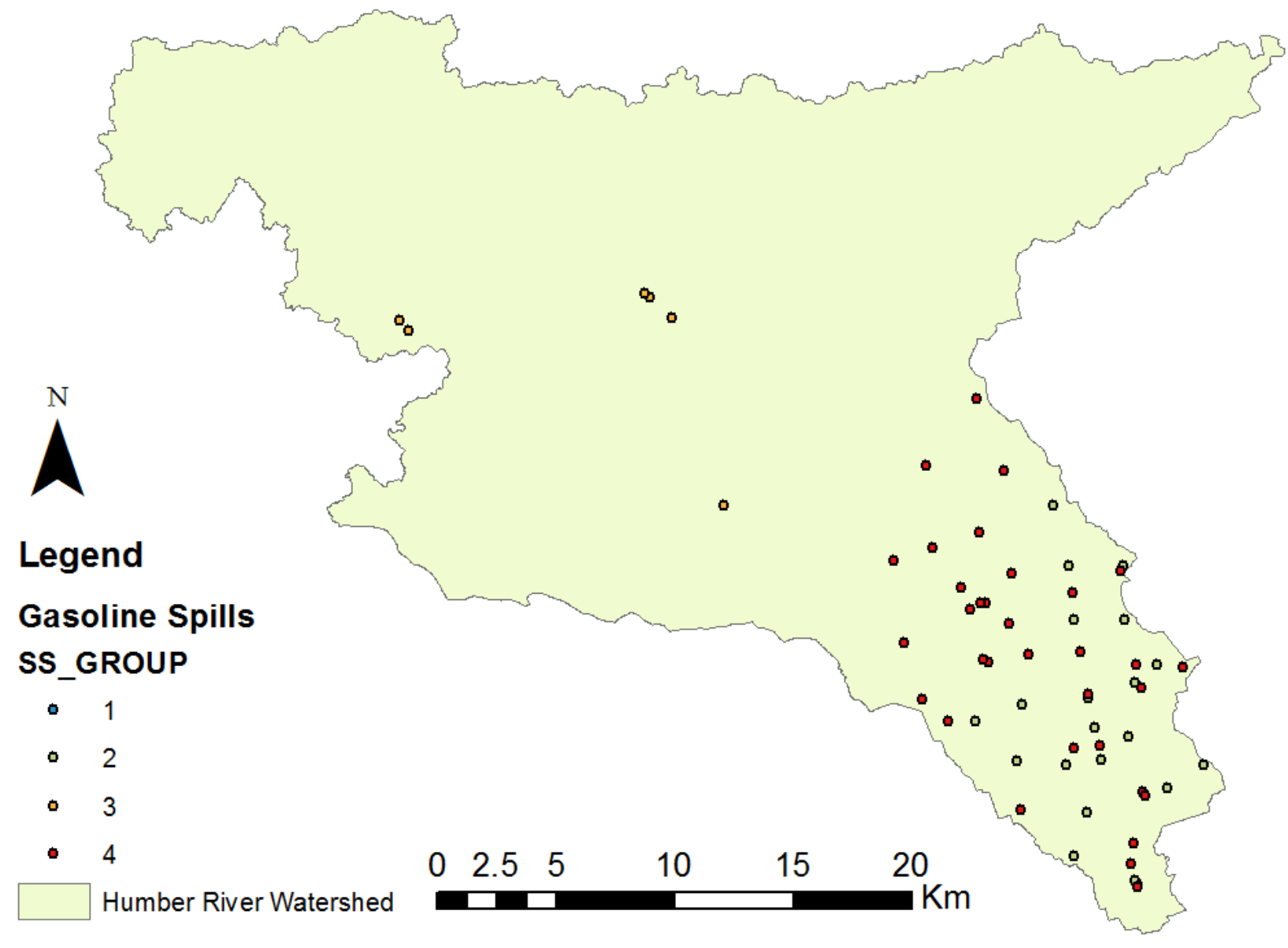

Figure 23. Groups of gasoline spill points with similar characteristics shown as colour-coded groups. 
As a result, 89 gasoline spill records within the Humber Watershed were extracted from the stationary spill sub-database to form the input simulation data set. The spatial statistical technique of grouping analysis was used to group features within the study area that are most alike. Various combinations of the number of groups, variables and parameter options were explored to yield the selected illustration of 4 groups of colour-coded points in Figure 23. Since the number of spill points is not high, four groups were selected to aggregate the points. The map of the input simulation data set in Figure 23 illustrates that none of the spill points are in exactly the same spot. It can be inferred from this map that there is no auto-correlation in the input data set. Some correlation was found between the geographic coordinates of latitude and longitude due to the nature of the relationship.

Gasoline spill records were placed into one of four groups based on similarities in Spill Mass (kg), \% Clean-Up and the spatial coordinates of Latitude and Longitude. The remaining numeric variable, IET (days), was not used because it is a spill characteristic that is derived from a series of spill records that does not originate with each spill record itself. The $\mathrm{K}$ nearest neighbour method was selected as the spatial constraint. The clusters of points in Figure 23 demonstrate the influence of the Longitude and Latitude variables because points of the same colour are generally placed near each other.

Since groups of spill points located more closely together are more similar, with respect to the numeric characteristics (except IET (days), gasoline spills in the lower basin area of the Humber River Watershed are more similar to one another than spills in the upper 
or northern part of the watershed. The investigation was then further specified to the lower or southern area of the watershed in which the majority of spills are located.

\section{Gasoline Spills in Lower Region of Humber Watershed}

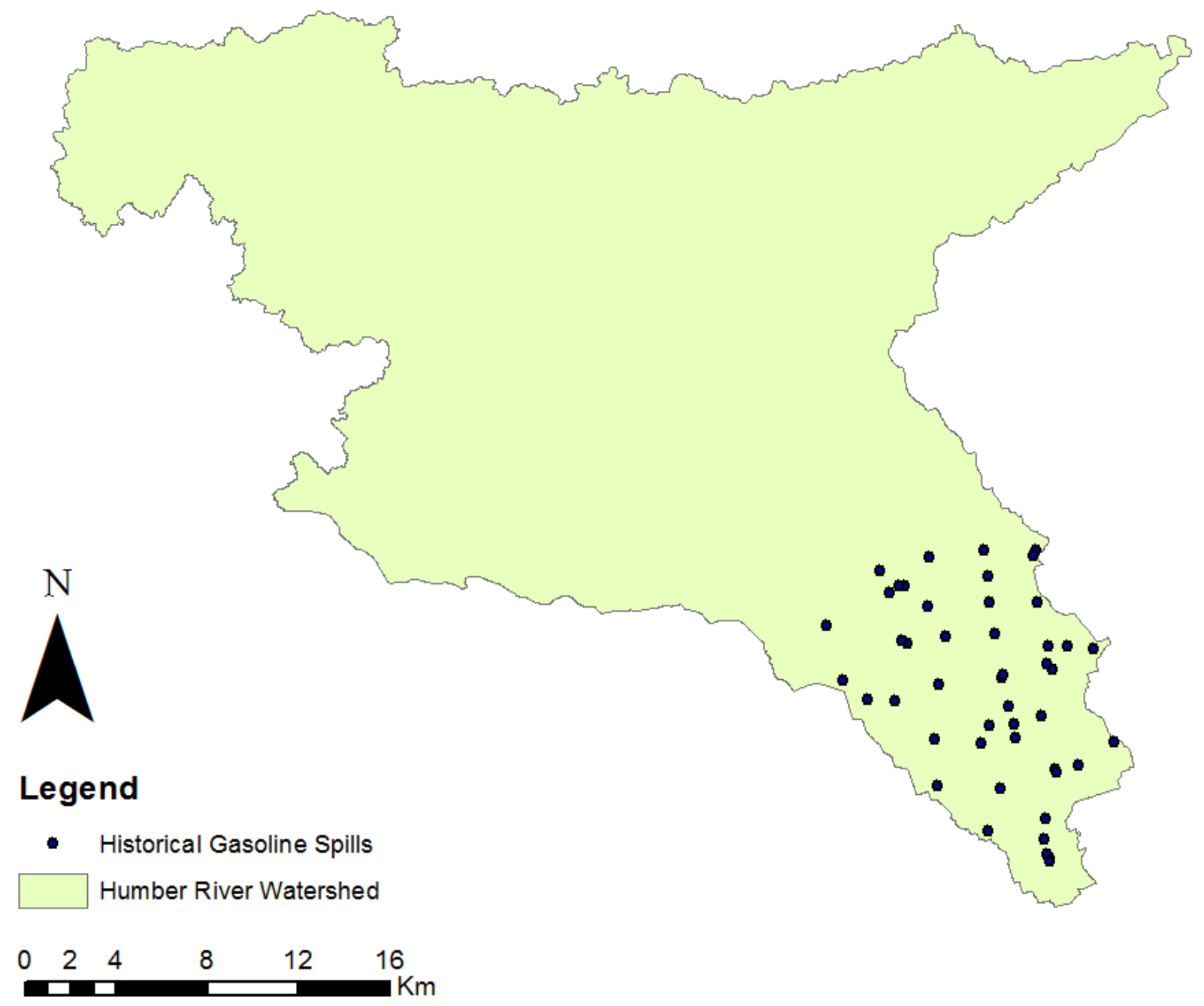

Figure 24. Lower region of Humber Watershed within the City of Toronto.

Figure 24 illustrates how the majority of gasoline spills (70 of the 89 historical gasoline spills) spills are situated within this lower region within the City of Toronto. The concentration of gasoline spills within the lower region can be explained by the greater 
concentration of industry and businesses in this area. The given DEM does not cover the entire Humber River Watershed which is also why the study area was slightly adjusted.

Since the adjustment was made later in the study, the input simulation data set of 89 historical gasoline spills was still maintained for the purposes of macro development. It was intended that a bootstrapping component be developed later as part of the macro program to generate sampling distributions. In order to apply the Central Limit Theorem, as many records as possible were maintained (Ahmed et al., 2014).

The IET (days) variable was re-calculated for the input simulation data set and does not contain any zero values, meaning that no two spills among the 89 gasoline spills recorded occurred on the same day. The $\%$ clean-up variable with no zero values was also used as well as the spill mass $(\mathrm{kg})$ variable with a minimum quantity of $6 \mathrm{~kg}$. A table of descriptive statistics for the data set is shown next as Table 6 .

Table 6. Descriptive Statistics for the Historical Gasoline Spill Set of Humber River Watershed

\begin{tabular}{|l|l|l|l|l|l|}
\hline Variable & \multicolumn{1}{|c|}{ Valid N } & \multicolumn{1}{|c|}{ Mean } & Minimum & Maximum & $\begin{array}{l}\text { Standard } \\
\text { Deviation }\end{array}$ \\
\hline IET (days) & 88 & 106.28 & 4.00 & 1009.00 & 140.45 \\
\hline $\begin{array}{l}\text { Spill Mass } \\
\text { kg) Plus 6 } \\
\text { kg }\end{array}$ & 89 & $2,233.49$ & 6.00 & $180,006.00$ & $19,096.14$ \\
\hline $\begin{array}{l}\% \text { Clean-Up } \\
\text { Plus 1 \% }\end{array}$ & 89 & 36.84 & 1.00 & 100.00 & 44.89 \\
\hline Longitude & 89 & -79.54 & -79.87 & -79.40 & 0.08 \\
\hline Latitude & 89 & 43.75 & 43.65 & 43.90 & 0.06 \\
\hline
\end{tabular}

The valid number of cases or spill records included in the calculation of descriptive statistics for the IET (days) variable is 88 and 89 for the other variables. This is due to 
the initial blank entry for IET (days) in the first spill record of the data set. IET (days) calculations are based on the difference between two consecutive values. Since the first spill record is not preceded by any other record, the initial IET (days) value remains blank. Only cases that do not contain any missing data for any of the spill records are included in the analysis for each variable (StatSoft, 2017).

With respect to the correlations of the variables, all correlations are calculated by excluding cases that have missing data or blank entries for any of the selected variables in order to base all correlations on the same data set (StatSoft, 2017). The correlation matrix shown in Table 7 is based on the 88 valid cases with no missing data for all variables of the input simulation data set.

Table 7. Correlation matrix for numeric variables of the input simulation data set.

\begin{tabular}{|l|l|l|l|l|l|}
\hline & IET (days) & $\begin{array}{l}\text { Spill Mass } \\
(\mathbf{k g}) \text { Plus 6 } \\
\text { kg }\end{array}$ & $\begin{array}{l}\text { \% Clean-Up } \\
\text { Plus 1 \% }\end{array}$ & Longitude & Latitude \\
\hline IET (days) & 1.000000 & -0.094978 & 0.015856 & -0.164021 & 0.147150 \\
\hline $\begin{array}{l}\text { Spill Mass } \\
\text { (kg) Plus 6 } \\
\text { kg }\end{array}$ & -0.094978 & 1.000000 & -0.112783 & 0.098921 & 0.050477 \\
\hline $\begin{array}{l}\text { \% Clean-Up } \\
\text { Plus 1 \% }\end{array}$ & 0.015856 & -0.112783 & 1.000000 & 0.110973 & -0.157289 \\
\hline Longitude & -0.164021 & 0.098921 & 0.110973 & 1.000000 & -0.653994 \\
\hline Latitude & 0.147150 & 0.050477 & -0.157289 & -0.653994 & 1.000000 \\
\hline
\end{tabular}

The correlations highlighted in blue in Table 7 are significant at $p<0.05000$. The only variables demonstrating statistically significant correlations are Longitude and Latitude which is expected due to the intrinsic relationship between longitude and latitude. The 
correlation coefficients in Table 7 are called Pearson $r$, the most widely-used type of correlation coefficients (StatSoft, 2017).

The non-numeric or categorical variables of the input data set were analyzed using bivariate histograms. The correlation matrix essentially measures the relation between pairs of numeric variables. To investigate the correlation between pairs of non-numeric variables, bivariate histograms were developed as shown in Figure 25.

\section{Bivariate Histogram of Reason vs. Cause}

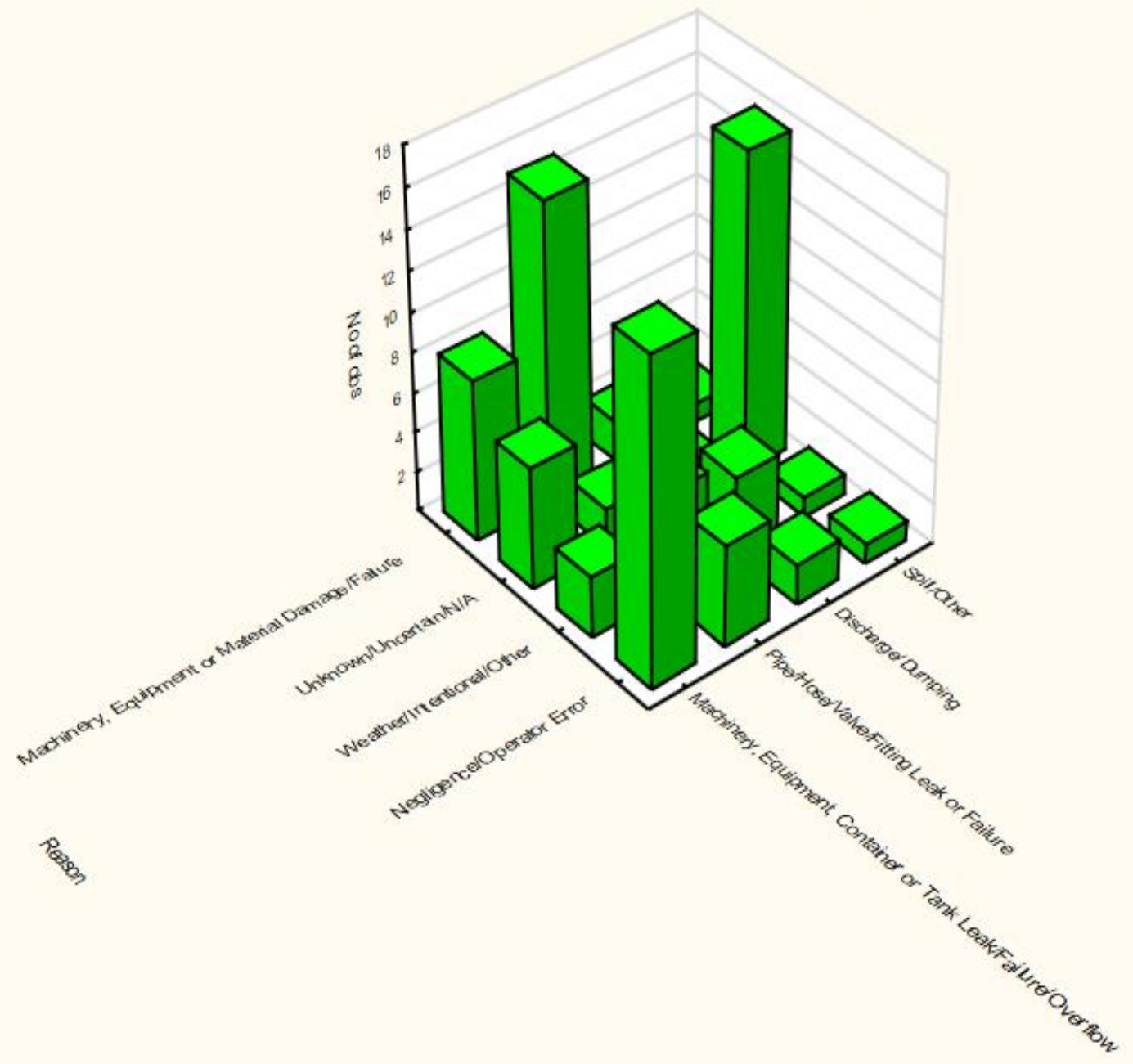

Figure 25. Bivariate histogram of categorical variables illustrating reason against cause. 
The bivariate histogram shown in Figure 25 illustrates the relation between the reason or explanation as to why a spill occurred against the sort or type of spill event that occurred. Figure 25 demonstrates that failure or damage of machinery or equipment is largely due to negligence or operator error rather than equipment or machinery failure or material damage/failure. Many preventative approaches can be derived from this finding to eliminate or reduce machinery or equipment failure.

Negligence or operator error also contributes substantially to pipe/hose/valve/fitting leak or failure. Since the Cause variable describes what happened, many spills were simply categorized as "spill" in the given data. This category indicates that the details of the spill event are unknown or the reason for the type of spill are unknown/uncertain/not applicable. Further results can be deduced from the bivariate histogram of Cause versus Sector as shown in Figure 26. 


\section{Bivariate Histogram of Cause vs. Sector}

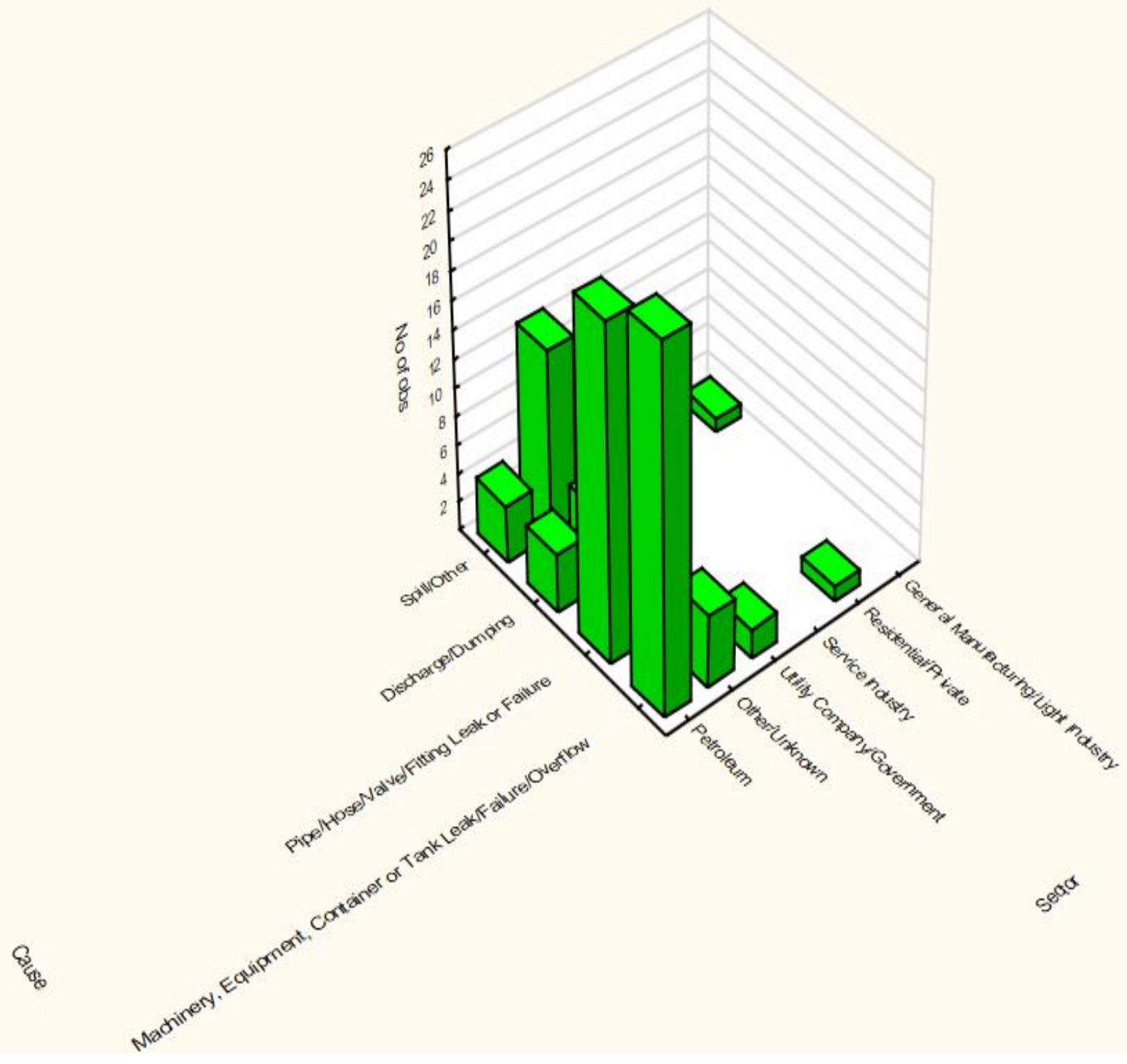

Figure 26. Bivariate histogram illustrating spill cause versus spill sector.

More specific suggestions for spill management solutions or strategies can be drawn from the bivariate histograms of the input simulation data set. For instance, the bivariate histogram in Figure 26 demonstrates how gasoline spills occurring in the Humber River Watershed mainly result from pipe/hose/valve/fitting leaks or failures and machinery, 
equipment, container or tank leak/failure/overflow within the petroleum sector. The results from the previous bivariate histogram in Figure 25 can be used to pinpoint reasons for machinery and equipment failures or breakage in the petroleum sector.

Negligence/operator error is a common reason for machinery and equipment malfunctioning or failure. Businesses in the petroleum sector can use this reasoning to improve their operations. An individualized approach could be taken by the petroleum company to reduce the likelihood of spills and to improve the control and response of spills when necessary.

\subsubsection{Macro Program Development \& Output Simulation Data Results}

A simulation or replication is defined as the execution of the outer-most loop in the macro program code to generate one series of predicted sequential spill records over an estimated time frame or planning horizon. The data series of sequential spill records is stored in a results spreadsheet output file. The number of simulations or replications to repetitively execute is defined in the macro program code and after one set of the simulations is generated, a "run" has been performed.

The spill data set containing 1,328 gasoline spills for Ontario was used in an earlier edition of the macro program to determine the minimum number of simulations necessary to repeat the looping system before the number of spills predicted in each results spreadsheet stabilized. The earlier version of the macro program that was developed employed a similar looping structure to the final macro program except that the number of simulations was incremented by 1,000 for each run until a group of 25,000 simulations 
was attained in one execution of the looping system. This was repeated for each approximate planning time horizon of 10 and 20 years respectively to generate 650,000 results spreadsheets, one for each simulation or replication.

For each time frame, the number of spills or spill records generated in each spill series data set is recorded in a summary table for each group of runs. The average number of spills is then calculated and plotted on a graph against the number of simulations in the group of runs. The procedure is repeated for both the 10-year and 20-year time frames. The resulting graphs for both time frames are shown in Figures 27 and 28.

The graphs in Figures 27 and 28 were used to estimate the minimum number of simulations required to execute the looping system as a group of runs before the number of spills predicted in the resulting output stabilized. Each curve on the figures takes the shape of a "knee" and at the top part, where the points begin to level off, the point at which the number of spills predicted begins to stabilize can be seen. The top of the knee of both curves on both graphs was visually determined to occur at approximately 15,000 simulations. The minimum number of simulations to repeat the overall loop in one run for both the shorter and longer planning horizons is 15,000 . 


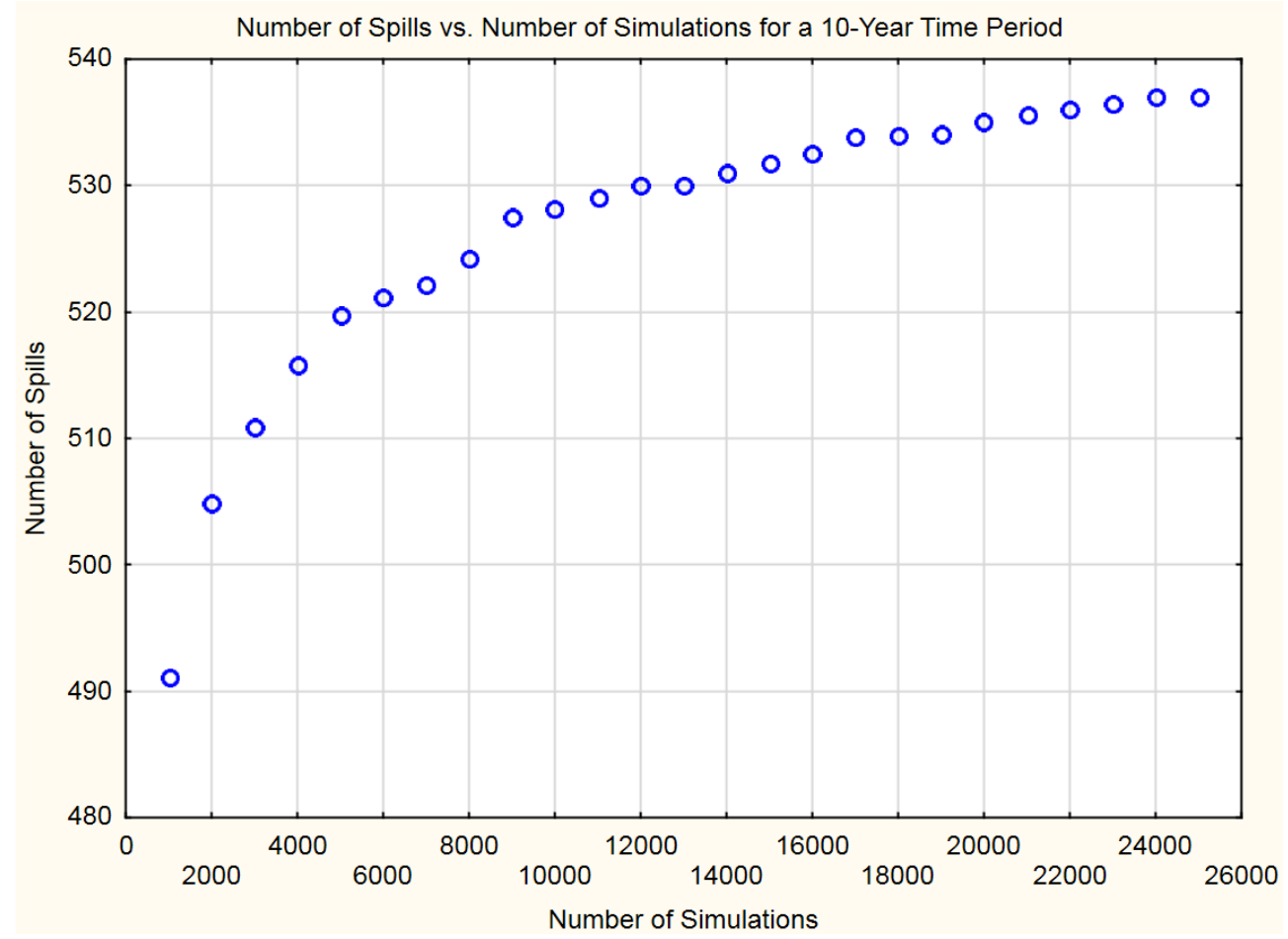

Figure 27. Number of spills vs. number of simulations over a 10-year time period.

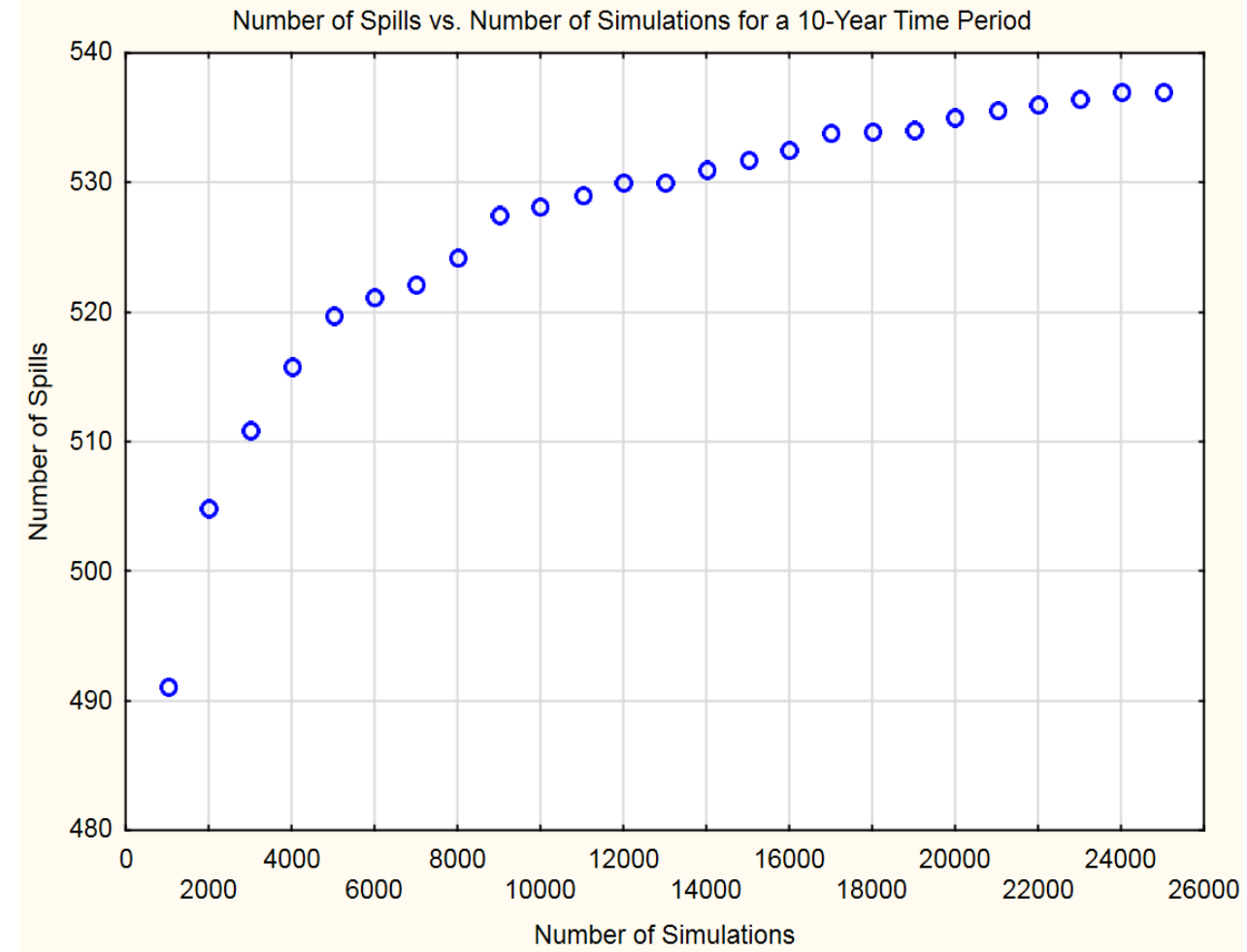

Figure 28. Number of spills vs. number of simulations over a 20-year time period. 
The macro was modified to reflect the fixed set of minimum simulation repetitions of 15,000 for each time period. The input simulation data set for gasoline spills within the Humber Watershed was used to run the macro and the number of spills predicted or spill records generated for each simulation was plotted against each simulation as shown in Figure 29.

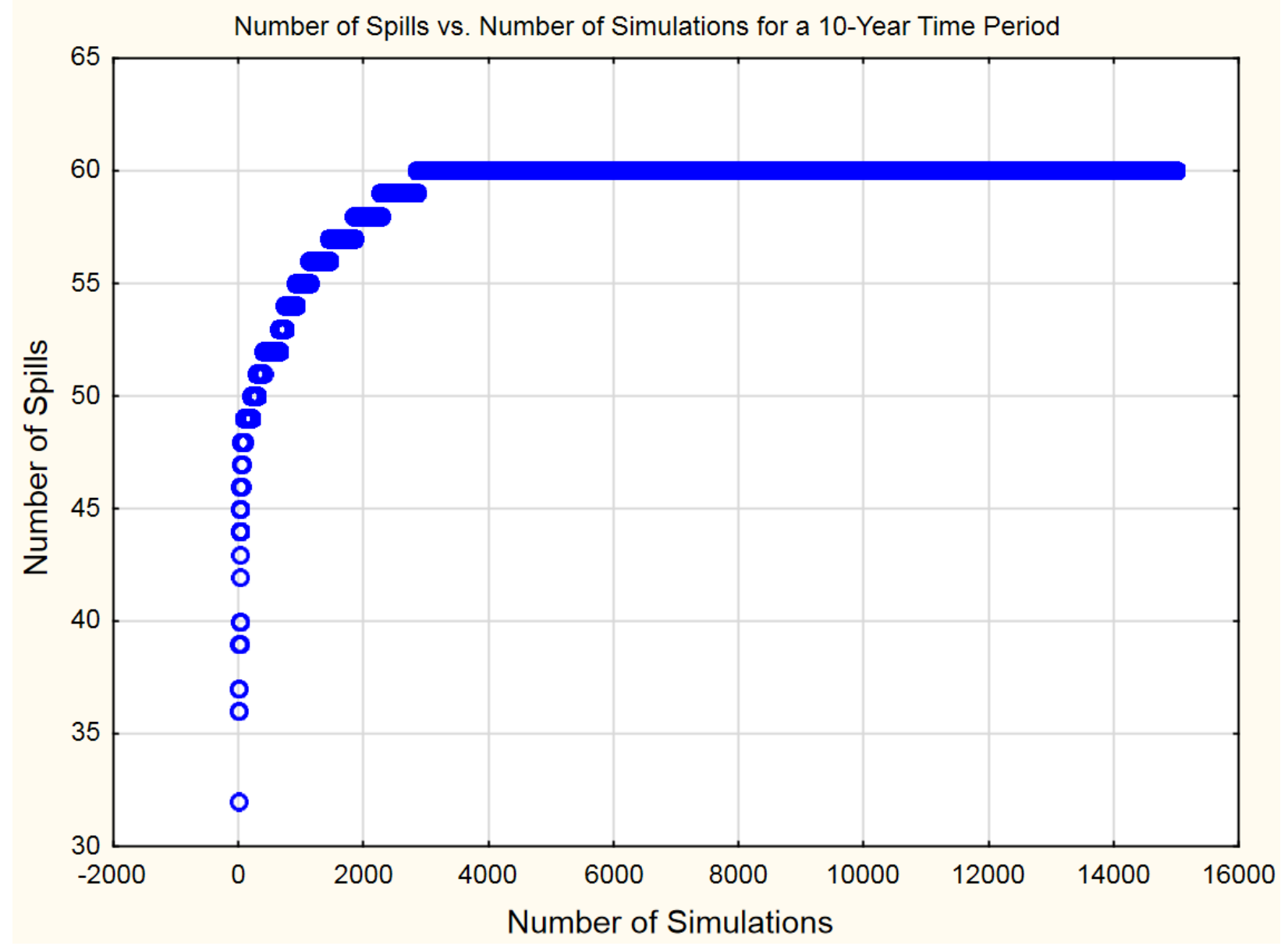

Figure 29. Number of spills plotted against the minimum number of simulations for the 10-year time period.

The graph in Figure 29 clearly illustrates that the number of spills predicted in each series of simulated spill data increases from approximately 32 for the first simulation to 60 at an approximate number of simulations of 3,000 and remains at that point thereafter. A 
similar result is found for the 20 -year time period. Therefore, the smaller data set may require a smaller number of simulation repetitions than the gasoline spill data set for Ontario that contains 1,328 records.

The output results are slightly different in the modified macro that employs the input simulation data set. The previous macro, that employs the province-wide gasoline spill data set, tabulates the mean and standard deviation of each of the three primary numeric variables (Spill mass (kg), Interevent Time (days), and \% Clean-Up) and the number of spills for each simulation in each set of repetitive simulations. Each set of simulations corresponds to a summary table and column statistics are used to calculate the average of each column of tabulated values to plot against the number of simulations in a set.

The final macro contains a fixed set of 15,000 repetitive simulations for each time period. The same summary table is developed as for the previous macro, however only one table is developed for each time period. Each table contains 15,000 rows of mean and standard deviation values for each of the three numeric variables in addition to the spill record count in the final column. Figure 30 illustrates the mean Interevent Time (days) values for each simulation. 


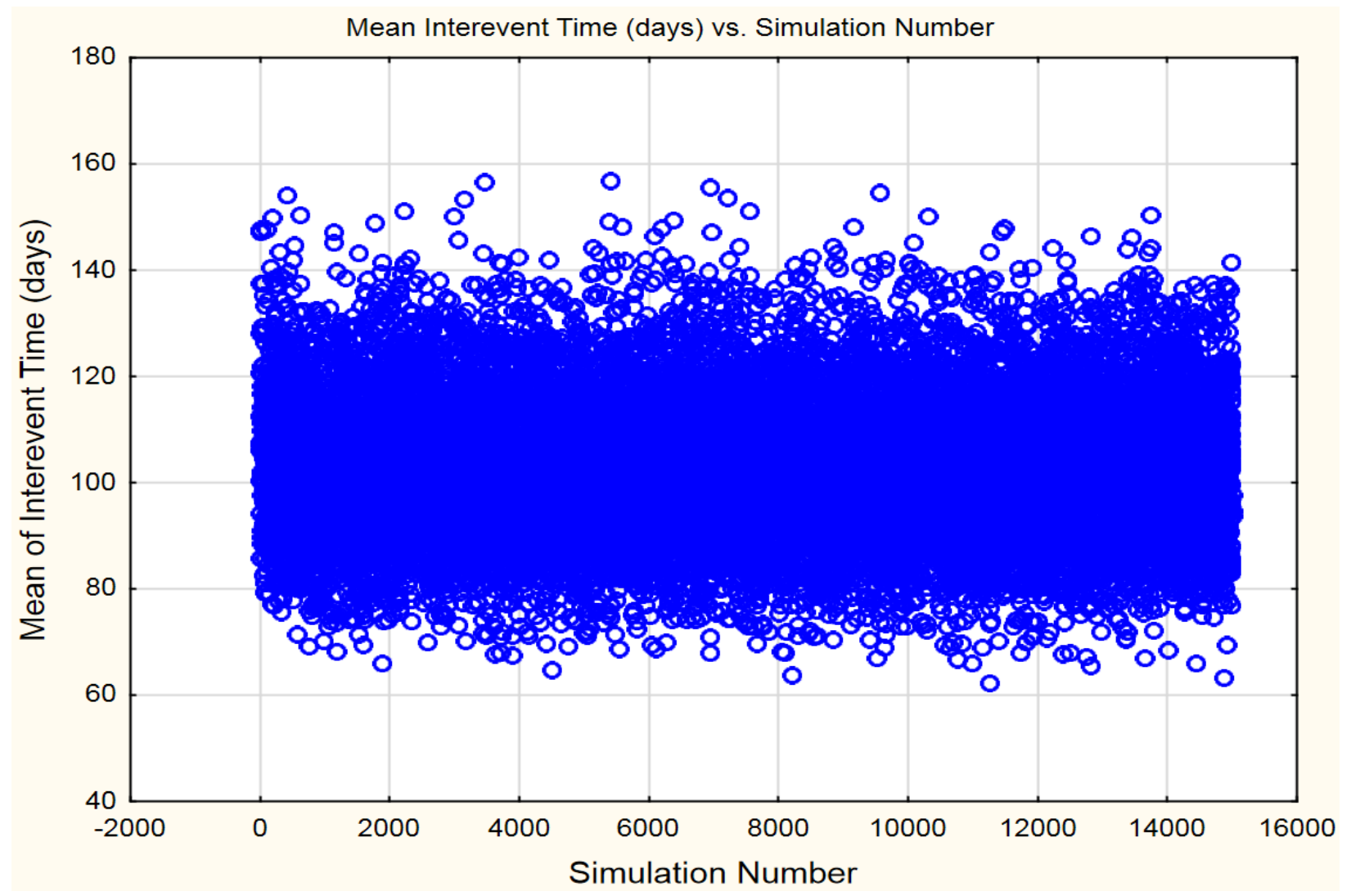

Figure 30. Illustration of mean Interevent Time (days) for each of 15,000 simulations. Interevent time (days) values are not averaged but plotted directly from the summary table for each of the 15,000 simulations. Graphs for the other two numeric variables have the same appearance. The only noticeably clear trend is seen for the graph of the number of spills as shown in Figure 29.

The output of the final macro contains a three-column spreadsheet with the mean for each of the three numeric variables of each random sample drawn from each simulation results spreadsheet. As part of the bootstrapping component of the macro, a random sample of $50 \%$ of the spill records in each simulation results spreadsheet was selected (with replacement) to create a random sample spreadsheet for each simulation. 
For example, one of the first Monte Carlo simulation results spreadsheets is comprised of 32 spill records and the associated random sample spreadsheet contains 16 spill records that were randomly selected from the simulation results spreadsheet. The random sample spreadsheet consists of approximately half of the spill records for each simulation results spreadsheet. It contains six columns of numeric variables (Interevent Time (days), Spill Mass (kg), \% Clean-Up, Rounded Interevent Time (days), Longitude, Latitude) and eight columns of categorical variables (Region, Source Type, NAICS Subsector, Sector, Client Type, Cause, Reason, Nature of Impact). The category names for the categorical variables are represented by code values for each category known as text labels that were automatically assigned when developing the input data spreadsheet. An example of text labels for the cause variable categories is given in Table 8 .

Table 8. Text labels for Cause variable categories.

\begin{tabular}{|l|l|}
\hline \multicolumn{1}{|c|}{ Text Label } & \multicolumn{1}{c|}{ Numeric Code } \\
\hline Machinery, Equipment, Container or Tank & 101 \\
\hline Leak/Failure/Overflow & \\
\hline Pipe/Hose/Valve/Fitting Leak or Failure & 102 \\
\hline Spill/Other & 103 \\
\hline
\end{tabular}

The mean of each column for Interevent Time (days), Spill Mass (kg), and \% Clean-Up are calculated for each random sample spreadsheet and placed in the aggregate table 
output for each time period. A descriptive statistics analysis is performed on the aggregate table output (with respect to the second, longer time period) for each of the three variables. A histogram of each variable within the aggregate output table is produced which represents the sampling distribution for the variable means of the Interevent Time (days), Spill Mass (kg), and \% Clean-Up values in the random samples. The sampling distribution or histogram for each of the three variables is displayed in Figures 31, 32, and 33.

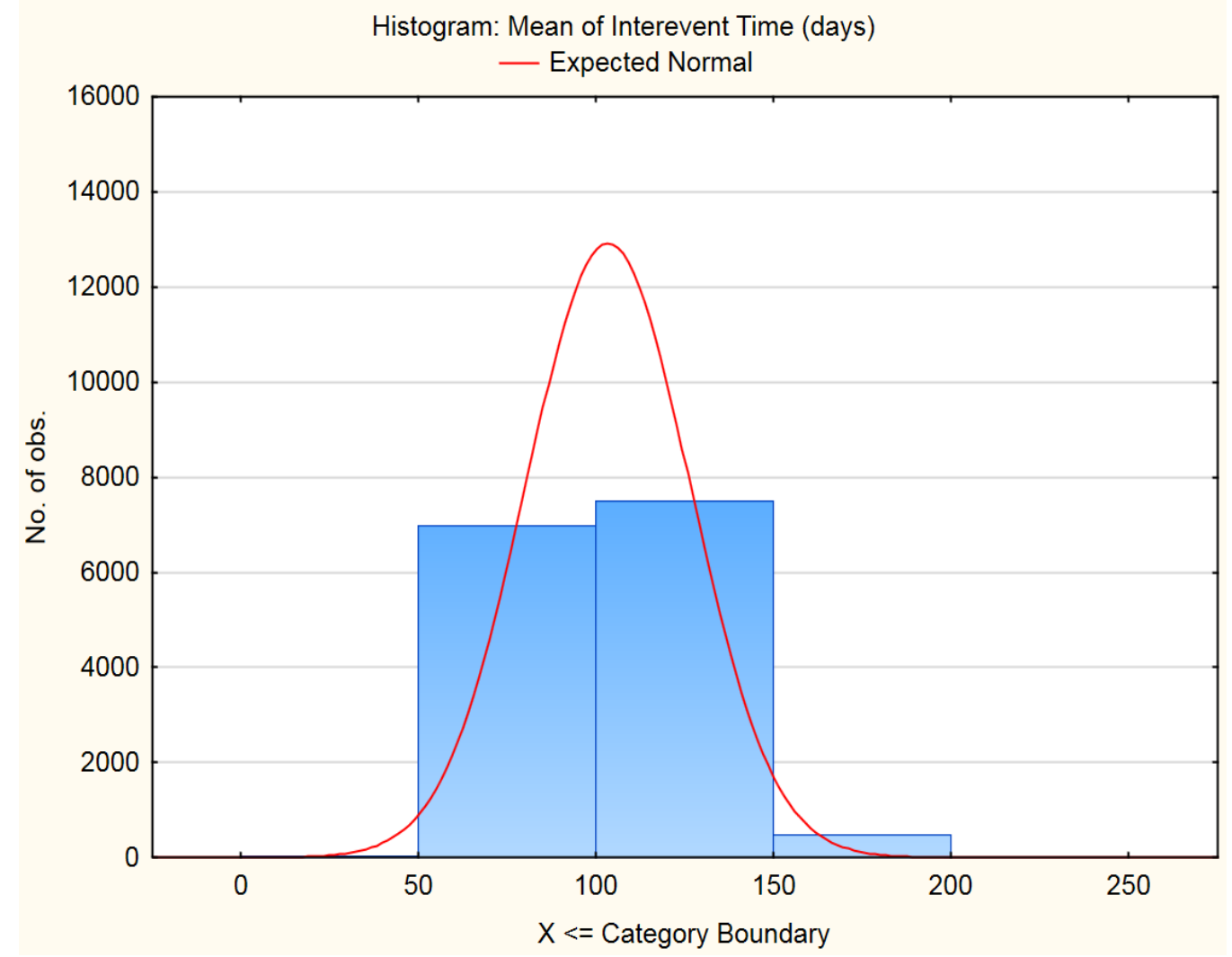

Figure 31. Histogram of mean of Interevent Time (days) for each random sample. 


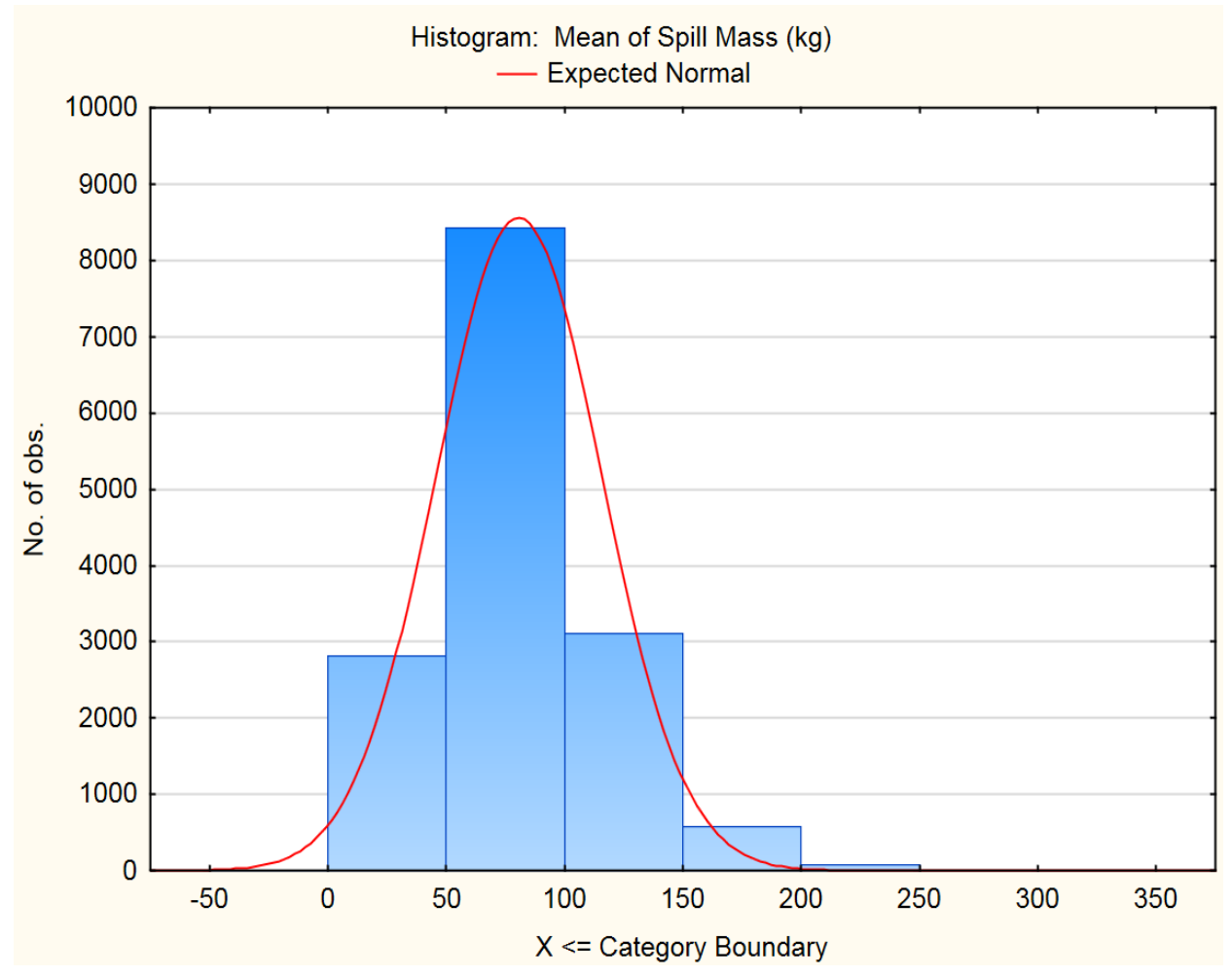

Figure 32. Histogram of mean of Spill Mass $(\mathrm{kg})$ for each random sample.

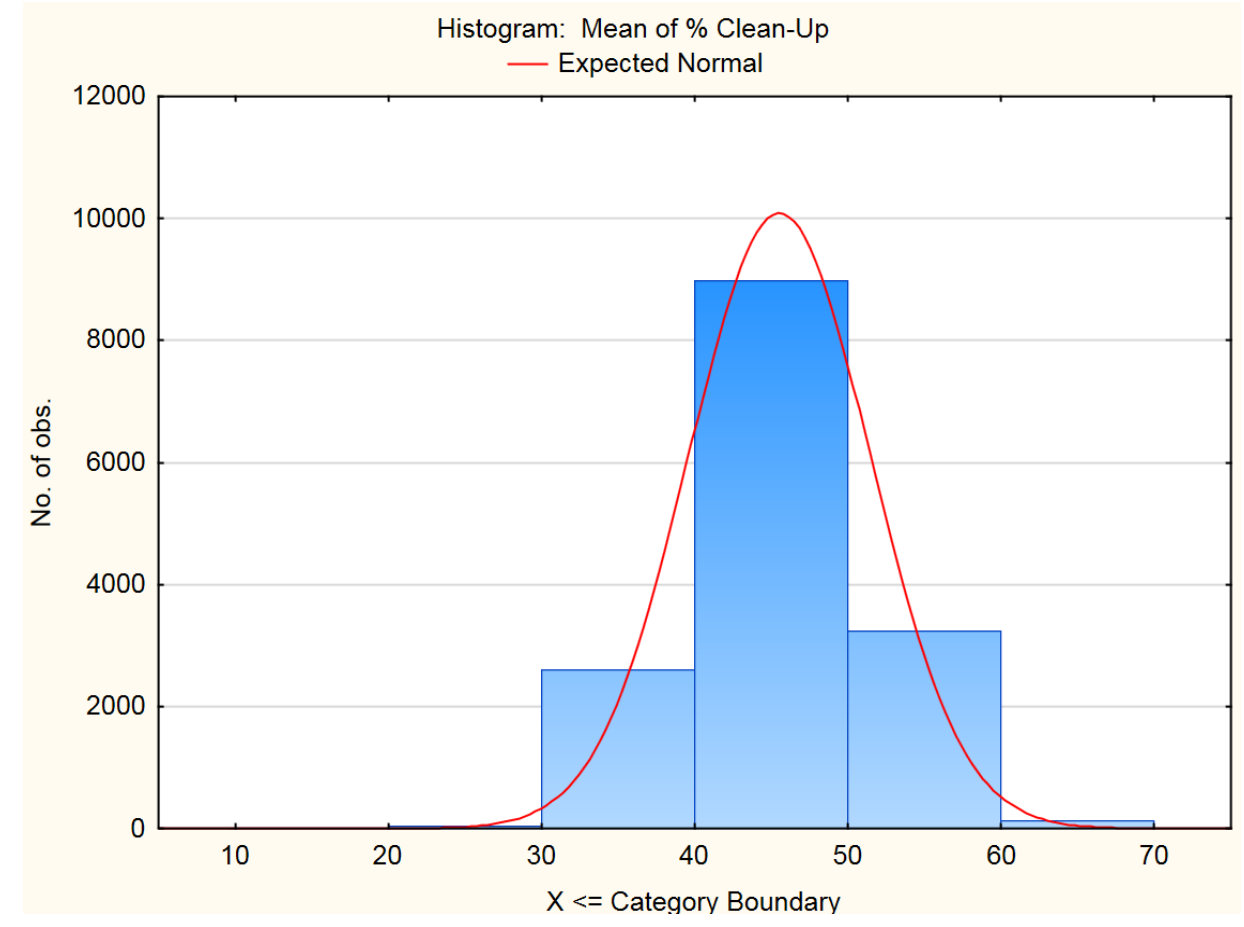

Figure 33. Histogram of mean of $\%$ Clean-Up for each random sample. 
Figures 31,32 , and 33 provide an estimate of the sampling distribution of the mean of Interevent Time (days), Spill Mass (kg) and \% Clean-Up based on the data. The distribution of the sample means in Figures 32 and 33 follow a normal distribution very closely. The resulting histograms of the bootstrap statistics provide an estimate of the expected distribution of the statistic and confirm the validity of the bootstrapping procedure employed.

A first-level sensitivity analysis was performed to investigate the effect of incorporating the correlation between the longitude and latitude values into the simulation procedure. The entire code of the macro program remained the same except the simulation method was changed from Monte Carlo to Iman Conover. The modified macro program was run with the same input simulation data set and nearly identical bootstrapping results were achieved as shown in Figures 31, 32, and 33. It is likely that the change in simulation method had little impact since there is no correlation for most of the numeric variables.

Pre-analyses of input simulation data variables were conducted to verify and support decisions and resulting selections of best-fitting distributions of each variable within the macro program. Univariate histograms were developed for each variable and a variety of distributions were applied to determine which distribution most closely approximated the shape of the histogram. The distributions of best fit were compared with the ranking of distributions by the distributions module within the macro program and a decision was made as to which distribution most closely approximated the empirical distribution of the variable. 
The selection of the distribution of best fit is an important step in simulation analysis since the simulated data and predicted values are drawn from the selected distribution. For the Interevent Time (days) and Spill Mass (kg) variables, the distribution which most closely fit is the log-normal distribution which was also selected by the program module as the best-fitting distribution. Figure 34 illustrates the log-normal distribution as applied to Interevent Time (days).

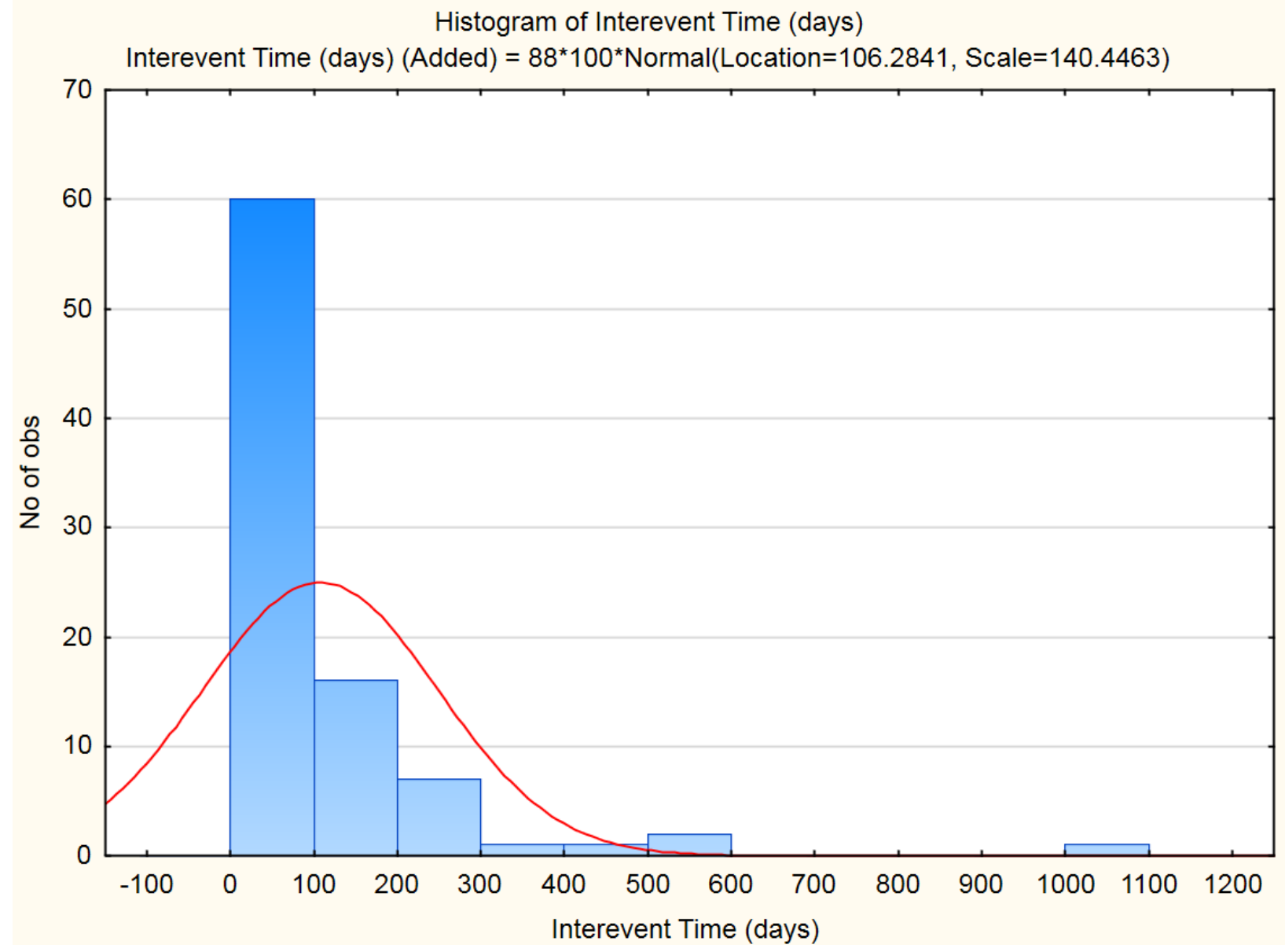

Figure 34. Log-normal distribution overlaid on histogram of Interevent Time (days). Although the log-normal distribution applied to the histogram of Figure 34 may not appear to be a perfect fit, it was pre-determined to be the distribution of best fit based on the pre-analysis as described above. 
For the \% Clean-Up variable, the three highest ranking distributions, by the distributions module within the macro program, are: (1) general pareto, (2) Gaussian mixture and (3) normal. The normal distribution was programmed as the user-defined distribution for $\%$ Clean-Up based the fit of the normal distribution to the histogram for \% Clean-Up as shown in Figure 35.

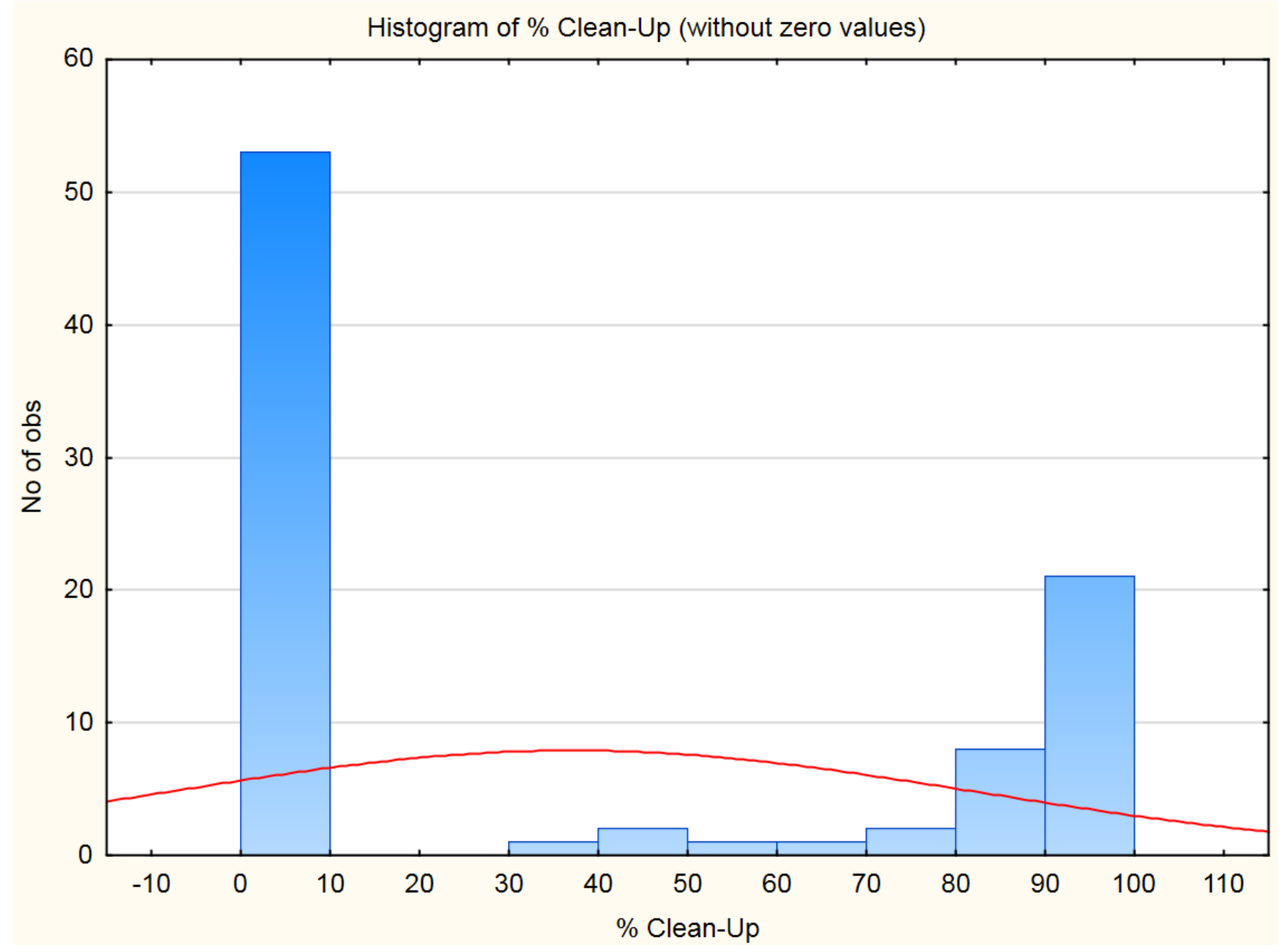

Figure 35. Histogram of \% Clean-Up with a normal distribution fit type.

Although the normal distribution in Figure 35 does not fit that well, it approximated the histogram much better than other distributions, including those ranked by the module. It 
was therefore selected as the best-fitting distribution for simulating future $\%$ Clean-Up data.

The macro program was edited to include a component for compiling the simulation results spreadsheets for each time horizon into a single, aggregate spreadsheet. The aggregate spreadsheet is an extended version of a simulation results spreadsheet that includes columns of continuous data from each simulation for each variable and statistic within the results spreadsheets.

Since each time frame generates 15,000 results spreadsheets, using either the Monte Carlo or Iman Conover method, a singular aggregate spreadsheet is comprised of the results from 30,000 simulation results spreadsheets. After the macro program was run with the latest component, two aggregate spreadsheets were generated that contain compiled results from 30,000 Monte Carlo simulation results spreadsheets for each time frame. The first aggregate spreadsheet contains 886,908 records of predicted spill data. The simulation output data was analyzed to develop and further refine spill management strategies. Descriptive statistics for Interevent Time (days), Spill Mass (kg), and $\%$ Clean-Up are given in Table 9. 
Table 9. Descriptive statistics for three numeric variables of aggregate output simulation data.

\begin{tabular}{|l|l|l|l|l|l|}
\hline Variable & \multicolumn{1}{|c|}{ Valid N } & \multicolumn{1}{|c|}{ Mean } & Minimum & Maximum & $\begin{array}{l}\text { Standard } \\
\text { Deviation }\end{array}$ \\
\hline $\begin{array}{l}\text { Interevent } \\
\text { Time (days) }\end{array}$ & 886,908 & 103.23 & 0.36 & 608.20 & 103.75 \\
\hline $\begin{array}{l}\text { Spill Mass } \\
(\mathrm{kg})\end{array}$ & 886,908 & 80.49 & 0.001 & 1308.88 & 155.24 \\
\hline \% Clean-Up & 886,908 & 45.59 & 0.0001 & 100.00 & 26.35 \\
\hline
\end{tabular}

According to Table 9, only approximately $46 \%$ of spills will be cleaned up in the next 15 years, on average, and the amount of clean-up may be negligible for some. This implies that more effort should be directed towards clean-up and remediation of spill events. Companies or organizations should familiarize themselves with clean-up techniques that are most suitable for their working environment and the oil types used. The aggregate output can also aid in the prioritization of spill management options. For example, since the projected $\%$ clean-up over the planning horizon is quite low, response and clean-up strategies should be made a priority over prevention and control strategies.

Table 9 indicates that future gasoline oil spills within fixed facilities may be very insignificant or very large with a maximum predicted spill mass of $1,308.88 \mathrm{~kg}$. Therefore, companies or people should be aware for possible large spill events and prepare themselves by storing clean-up kits in convenient and accessible locations. A 
minimum interevent time of 0.36 days is suggested as the possible minimum time in between spills events which means that more than one spill could happen on the same day as it has occurred historically, even possibly within the same location.

\subsection{Analysis of Spill Path \& Travel-Time Model Results}

Inland oil spills travel downslope over land and usually enter streams or other surface water features (Farrar et al., 2014). Surface water features can be entered into directly or through an outlet structure of the stormwater system. In urban areas, most spills travel over paved areas and enter into storm sewers, also known as storm drains, which convey surface runoff to an appropriate discharge location (Chin, 2013).

The lower region of the Humber River Watershed was used as the case study area to model the spill overland and storm sewer system pathways. To model the overland flow path, the least-cost path is calculated from the spill point to the access point of the storm sewer system, known as the sewer receptacle or manhole. Sewer receptacles or manholes provide access to sewer systems for preventative maintenance and emergency service and are usually located at the following points: junctions of sanitary sewers, changes of grade, size or alignment, and at the beginning of the sewer system (Chin, 2013).

Manholes or sewer receptacles serve only as access points and not entry points for surface runoff from urban streets which is usually routed to sewer pipes through street gutters and stormwater inlets (Chin, 2013). For the purposes of developing the model, manholes or access holes were the assumed entry points for surface runoff into the storm 
sewer system. The access points shapefile was the only data provided to model the junction points of the storm sewer system. Access points have been used in ArcGIS to model the street drains in order to determine the upstream entry point of a chemical spill before it entered the river (ESRI, 2017).

To model the overland flow path, which precedes the sewer system path, cost distance tools were used to calculate the least-cost path from the spill point to the sewer receptacle. Oil, when spilled, is much like water for it takes the path of least resistance while travelling in the steepest downslope direction. Cost distance tools were used to derive the path of least resistance down a DEM which is the starting point for the spill path and travel-time model.

The given DEM contains 3 metre by 3 metre raster grid cells which is relatively fine in comparison with other available DEM data sets. The fine resolution of the raster grid cells was well-suited for deriving the least-cost path since path width is typically around 1 metre and is not allowed to exceed the dimension of the land elevation grid cells (Farrar et al., 2014). The DEM is illustrated in Figure 36 after converting the elevation units and filling in sinks greater than 1 metre. 


\section{Digital Elevation Model (DEM) of City of Toronto}

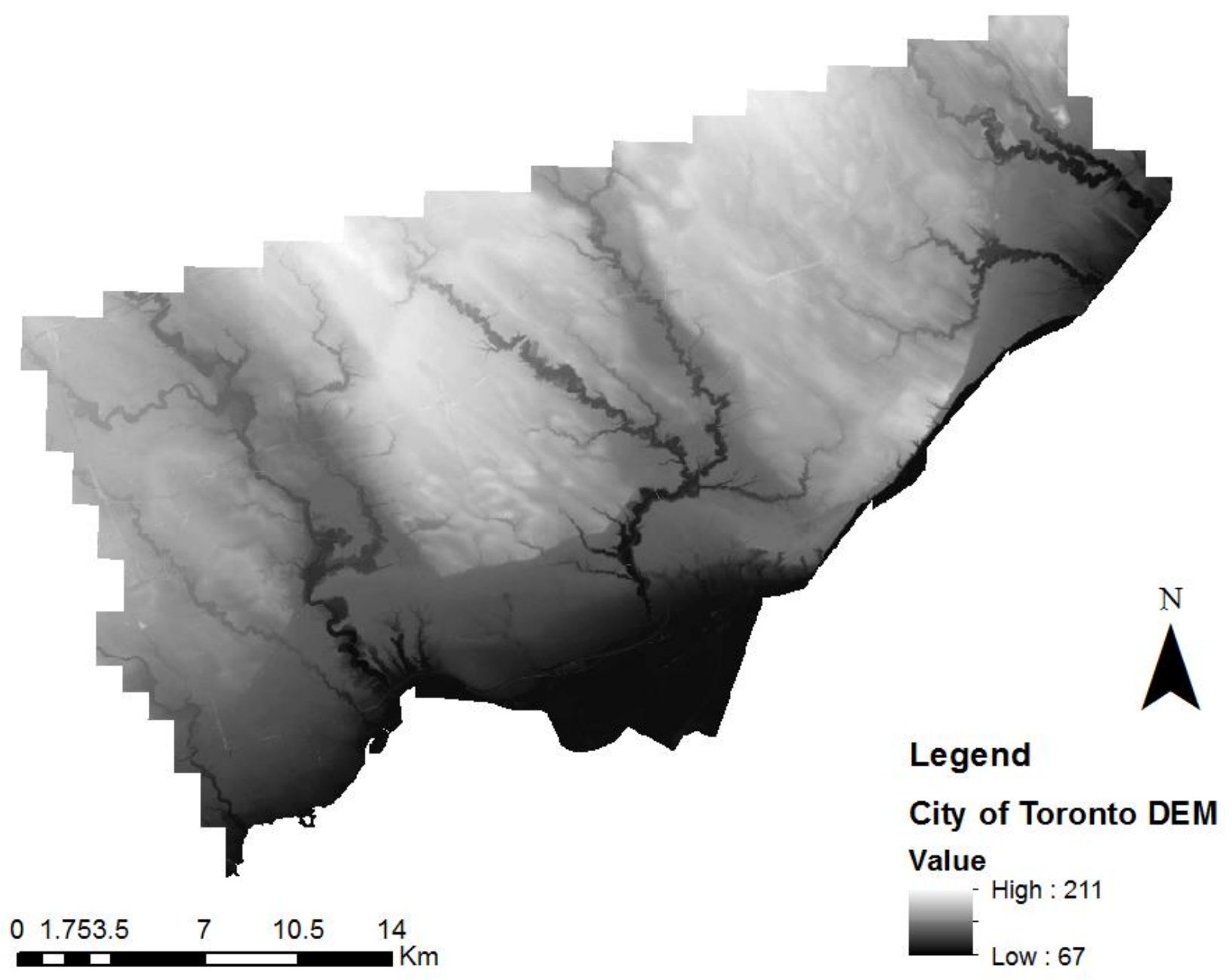

Figure 36. Illustration of Toronto DEM after initial adjustments.

Despite the adjustments made on the DEM, it still exhibits some oddities. For instance, there are often small groups of horizontal lines scattered throughout the image and it is unclear what these lines or little rectangles mean, resemble or represent. An example of this peculiar pattern is illustrated in Figure 37. 
Figure 37. An example of an odd grouping of lines seen throughout the DEM both before and after the DEM was modified.

The DEM is the input surface raster for many tools. A slope raster was derived using the DEM shown in Figures 36 and 37 as the input surface raster to produce a slope raster that identifies the slope, gradient or steepness, from each cell of a raster (ESRI, 2017). A portion of the slope raster is illustrated in Figure 38 in which the odd grouping of lines in Figure 38 is also noticeable in addition to 1 metre contour lines. The resulting slope raster is also a contour map with a 3 metre accuracy in the $\mathrm{x}$ and $\mathrm{y}$ direction and a one metre contour map combined. This result demonstrates that the given DEM is not a fully extended DEM. 


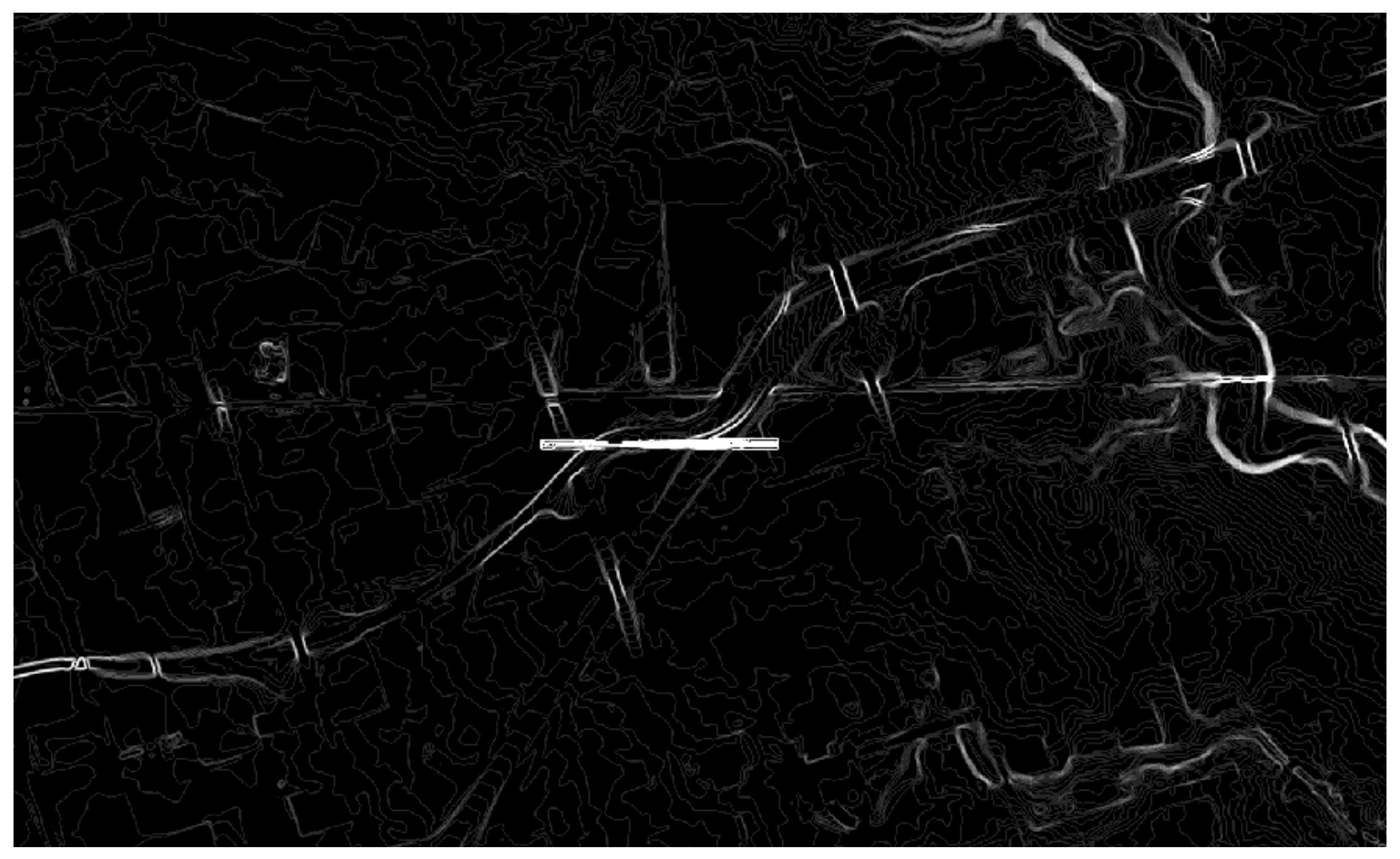

Figure 38. The slope raster resulting from the DEM input raster.

Although the DEM contains some oddities, it was still both the initial and central piece of GIS data throughout the study. It was used as the input raster of a continuous surface for the City of Toronto for many tools and modules within ArcGIS. One of its key usages was to create an output raster showing the flow direction from each cell to its downslope neighbour(s) using the D8 flow method. This output raster is known as the flow direction raster and served as the input for delineating all drainage basins in the DEM. A basin was delineated which coincided with the lower region of the Humber Watershed. The boundary of the basin was used thereafter as the study area and all GIS data sets used in the case study were extracted for this area. 
A simulation results spreadsheet was randomly selected and displayed using the longitude and latitude coordinates to produce a shapefile of predicted gasoline spills. Since the input simulation data set consists of gasoline spills for the entire Humber Watershed area, some of the predicted spills are located in the upper part of the Humber Watershed above the boundary for the City of Toronto. These spills were excluded and an extracted data set of 37 predicted spill points located in the lower basin area of the study area were used in the development of the model. The access points and sewer lines were also extracted to form subsets of points and lines for the lower basin area. Some important GIS data layers used for the model development are displayed in Figure 39.

\section{GIS Data Layers for Model Development}

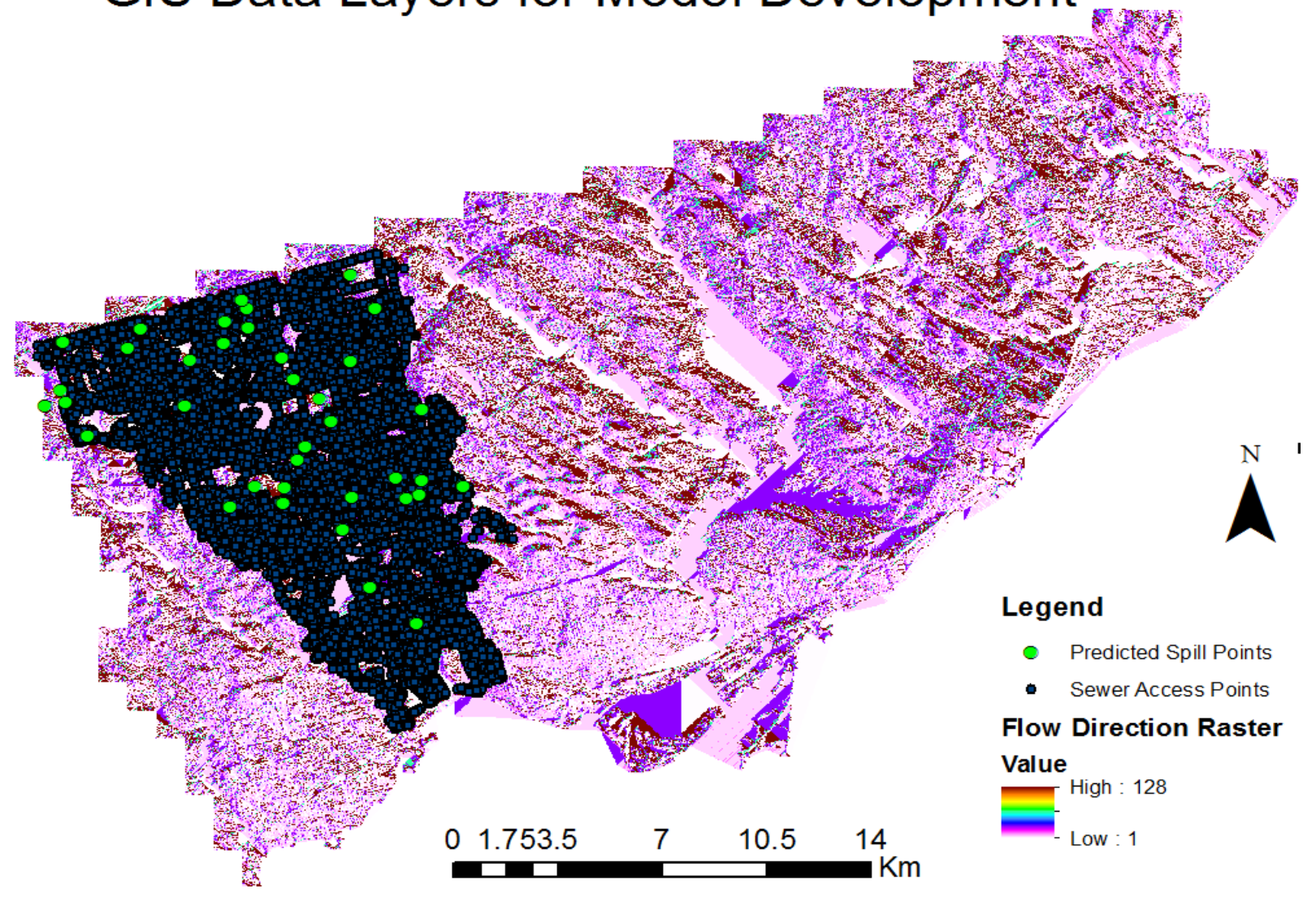

Figure 39. Some important GIS data layers used in model development. 
The multi-coloured, purple raster of Toronto is the output flow direction raster and the patch of different layers on the left side of the image contains the required data layers as extracted for the study area. The small blue points represent the access points and the bright green dots represent the predicted spill points. The sewer lines and the polygon shapefile layer of the lower basin area of the Humber Watershed are not included to simplify the map. However, the shape of the entire patch of GIS layers is the shape of the lower basin area.

A closer view of the GIS data layers is given in Figure 40 using a different set of colours to represent each feature layer.

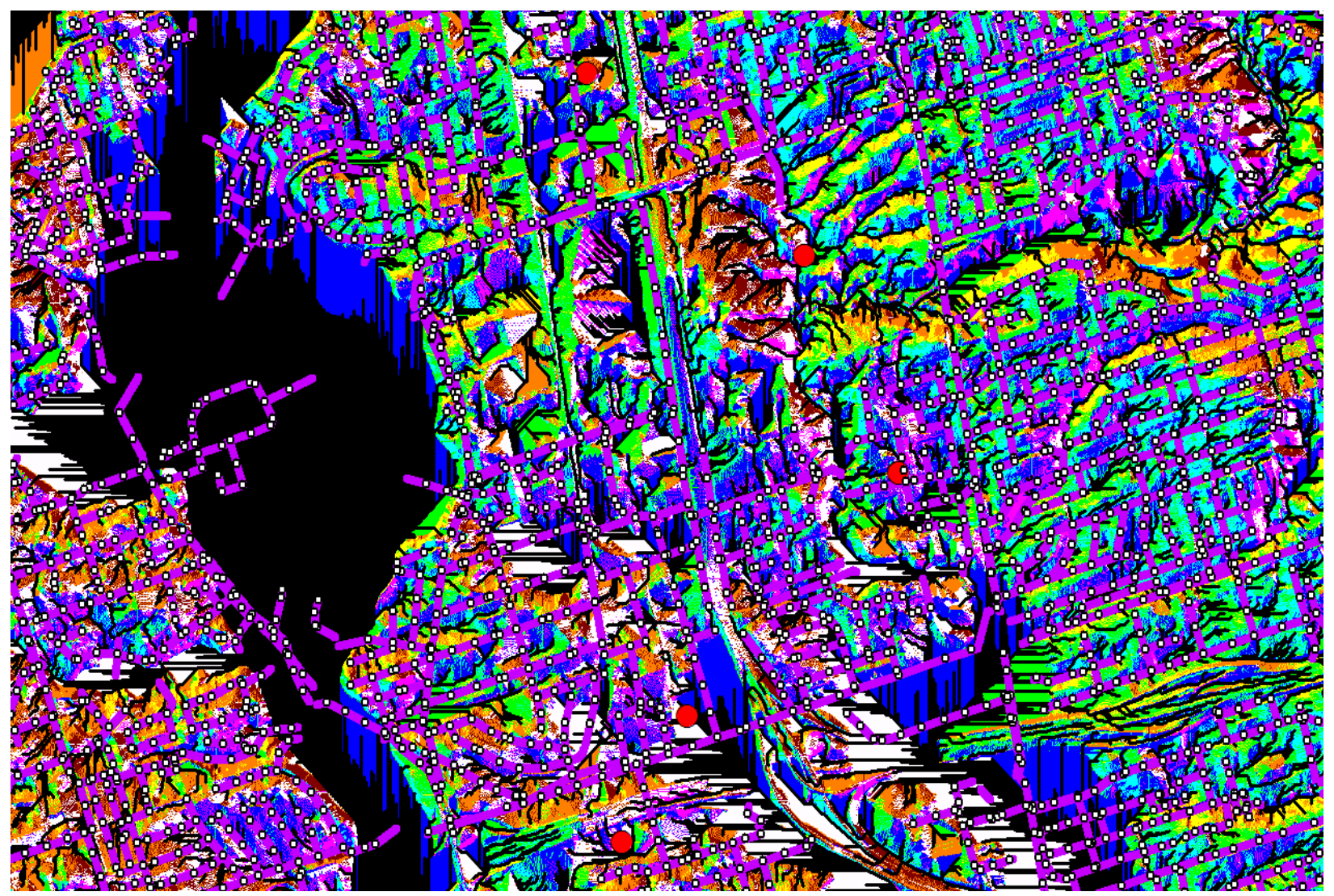

Figure 40. A close view of many GIS data layers used for model development. 
In Figure 40, the purple lines with beige dots represent the sewer lines and the access points respectively. The bright red dots represent the predicted spill points. The bright blue background is the study area or lower basin area of the Humber Watershed. The feature to the left with lines protruding from it is a vectorized stream network representing the Humber River. This polyline feature class was mainly used for visualization purposes. It was not used for model development. The general background consisting of a variety of colours is the flow direction raster. The illustration in Figure 41 presents a magnified view of an individual spill point from Figure 40 and its surrounding environment. The path and travel-time for each spill was initially delineated for each individual spill point using the direction coding legend shown in the upper left-hand corner of the image and spreadsheet-based calculations.

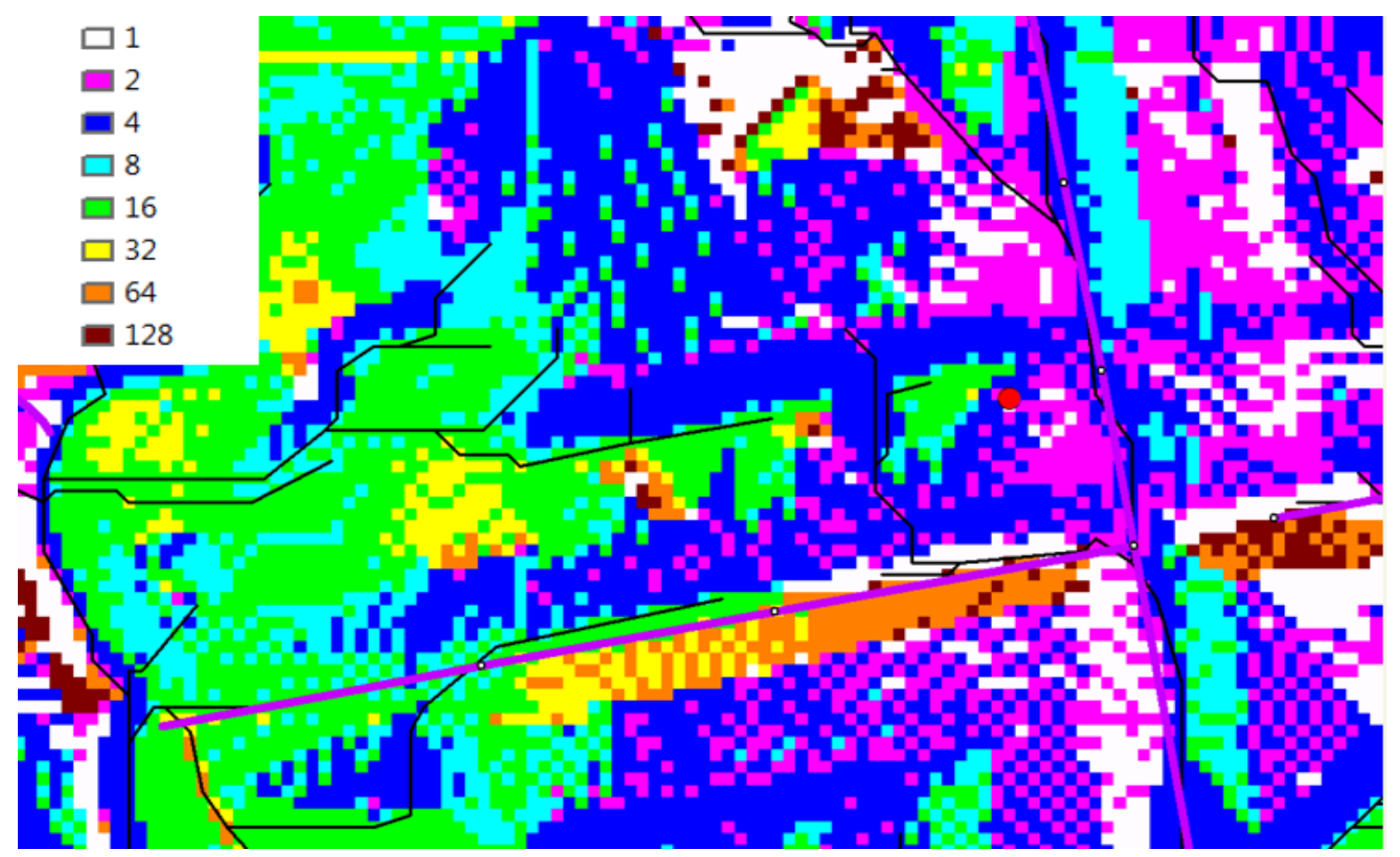

Figure 41. An individual spill point and the direction coding legend for the underlying flow direction raster. 
The spill path was traced on a cell-by-cell basis from the spill point (red) to the access point of the sewer line (purple) using the colour-coded directions of the direction coding legend. The length of the pathway was measured and the average slope of the pathway was extracted from pixel values of the underlying slope raster layer. Time of concentration methods were used to calculate the overland travel-time of the spill. Time of concentration can be defined as the longest travel-time that it takes for surface runoff to reach the discharge point of a catchment (Chin, 2013).

The NRCS (Natural Resources Conservation Service) Method was used, for example, to calculate the overland flow time in two parts: first for sheet flow (flow lengths of approximately less than $100 \mathrm{~m}$ ) and then for shallow concentrated flow (remaining flow lengths) when applicable. Various values of Manning's roughness coefficient, $n$ (dimensionless), for overland flow were investigated although the total time of concentration was often determined to be quite long, sometimes several hours, which is unrealistic in most cases.

Using the spreadsheet-based approach, the access point was the ending point of the overland spill path and the beginning point of the spill path through the sewer system. The spill was traced through the sewer system based on the beginning and ending elevation points of each sewer line segment. The channel flow time was calculated for each sewer line segment using equation (8) and summed for the entire sewer line pathway while assuming full-pipe flow conditions. The assumption of full-pipe flow conditions is appropriate for this analysis and model because it provides the shortest time of flow for 
the spill through the pipe, which results in the fastest travel-time. The fastest travel-time provides the most conservative estimate for spill response which ensures or at least encourages a timely response to the potential spill site upon the exit of the spill from the storm sewer system.

The summation of channel flow time for each sewer line segment resulted in fairly realistic values of channel flow times, from approximately one minute to twelve minutes. The overland spill path travel-times were much higher than reasonably expected, from approximately five minutes to several hours, when a practical assumption for urban areas is often 5 minutes to 10 minutes. The spreadsheet-based approach, combined with the visualization of GIS data layers, was still considered useful as a preliminary step in understanding how to model spill pathways.

The limitation in using Manning's equation with constant $n$, equation (4), for overland spill routing is that it is conventionally used for water flowing in sewer pipes. However, since gasoline exhibits similar flow characteristics to water, it was applied to model gasoline spills in the case study. The equation has also been applied in GIS modelling to calculate the velocity (V) of the leading edge of the spill as it travels over the land surface (Farrar et al., 2014).

Manning's equation for full-flow conditions, equation (8), was applied to routing the spill through the storm sewer system. This equation is conventionally used to design storm sewers since most storm sewers are sized to flow full at the design discharge (Chin, 
2013). It is therefore directly applicable to the storm sewer system environment. By employing Manning's equation for full-flow conditions when determining the spill pathway through the storm sewer, the most conservative estimate for pipeline travel-time is obtained because the fastest travel-time is obtained. However, the estimates may differ from actual conditions since the storm sewer pipelines may be empty and dry - especially if a rainfall has not occurred for some time. The spilled gasoline or oil will travel more slowly under these conditions.

The development of an automated model tracing overland and storm sewer spill pathways was initiated based on the former approach, using the spreadsheet calculations and GIS visualization layers. The access points for the study area and the DEM shown in Figure 33 were used as inputs for a cost distance analysis tool. The cost distance analysis tool calculates the least accumulative cost distance for each cell to the least-cost destination point (sewer system access point) over a cost surface. The terms source and destination are often used interchangeably in the literature accompanying ArcGIS. For the purposes of this model, the set of predicted spill points is considered as the source feature class and the set of access points of the storm sewer system as the destination feature class.

The cost distance tool generates two significant output raster datasets, the output cost distance raster and the output cost backlink raster, as depicted in the next two figures, Figures 39 and 40. Figure 39 illustrates the output cost distance raster shown beneath the input shapefile of access points within the study area. 


\section{Cost Distance Output Raster with Sewer Access Points}

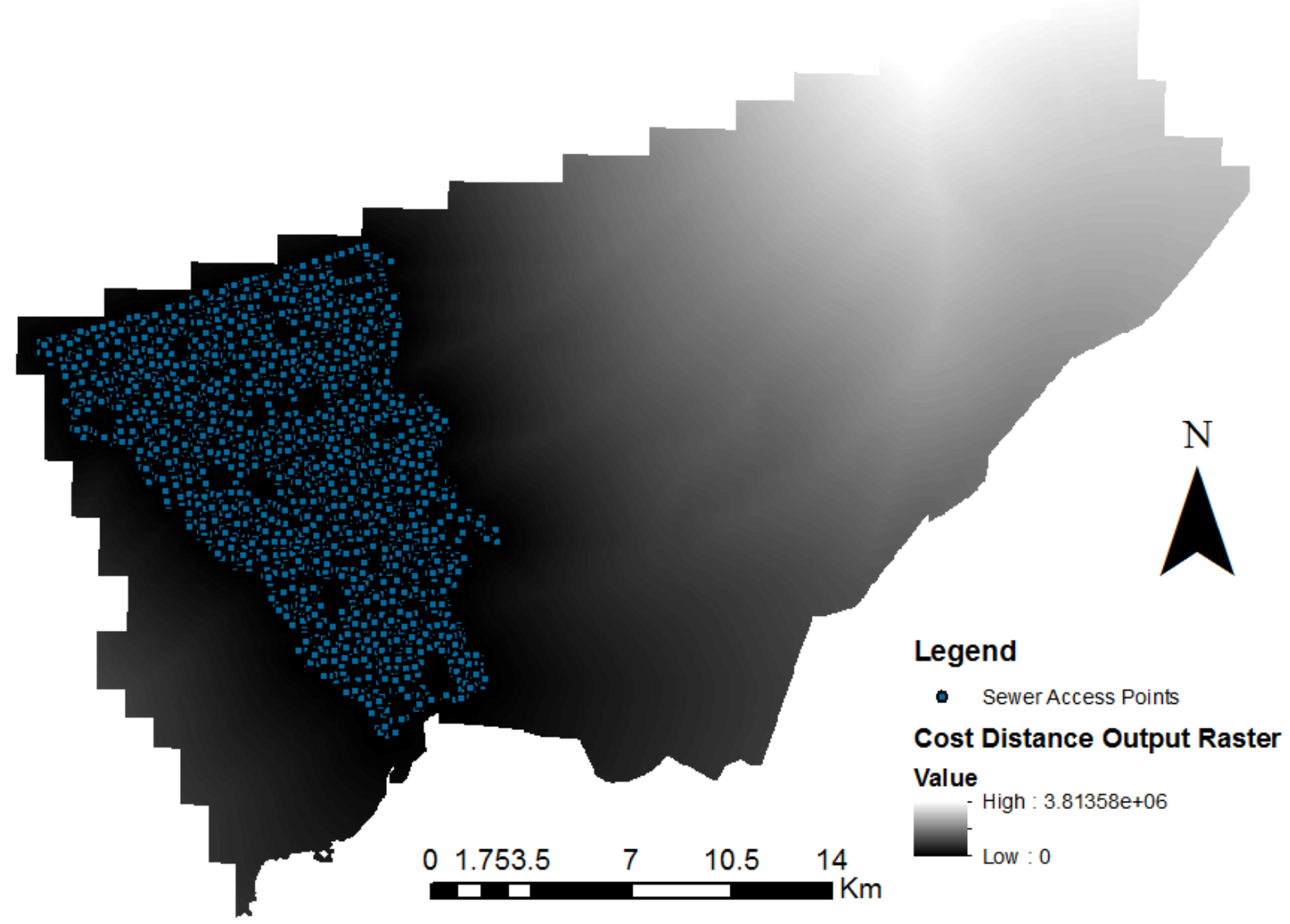

Figure 42. The output cost distance raster resulting from a cost distance analysis tool underlying the access points layer (blue points) for the study area.

It is interesting to note how the cost distance tool receives two input files with different coverage extents, one larger (the DEM) and one smaller (the access points shapefile) yet outputs a cost distance raster and cost backlink raster dataset with the larger coverage extent. Figure 40 illustrates the contrast between the colour scheme and pattern for the study area versus other parts of the image.

The cost backlink output raster of Figure 43 assigns values from zero to eight inclusive to each raster cell in a similar manner as the flow direction raster and accompanying 
colour-coded diagram of Figure 41. The zero grid cell occupies the central cell and represents the fixed position of the destination location or access point. The neighbouring eight cells of the central cell are assigned values 1 through 8 to identify the next neighbouring cell (the succeeding cell) along the least accumulative cost path from a cell to reach its least-cost destination (access point). If the path continues to the right neighbour, the cell will be assigned the value 1, then 2 for the lower right diagonal cell and then continuing in a clockwise manner.

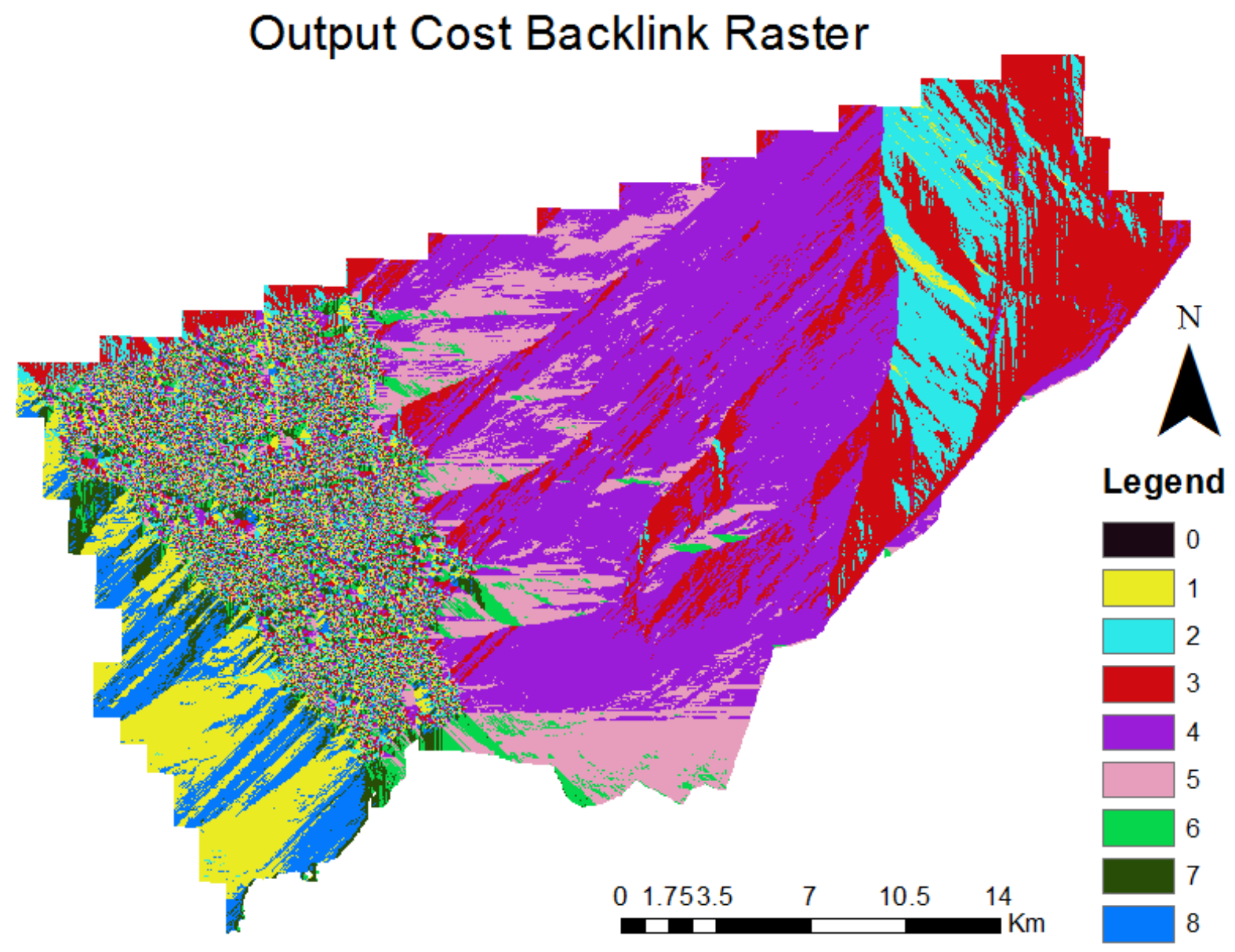

Figure 43. The output cost backlink raster resulting from a cost distance analysis tool. 
The assignment of values for the output cost backlink raster differs from the direction coding of the flow direction raster which does not assign any value to the central cell and does not include the central cell in the grid of the flow direction raster. The central cell of the output cost backlink raster represents the fixed destination points as provided by the access points shapefile layer used as input. The cost backlink raster essentially gives the neighbour that is the next cell on the least-accumulative cost path to the nearest destination or central cell, a value of zero.

The two raster datasets produced by the cost distance analysis tool then became inputs, in conjunction with the shapefile of predicted spill points, to the next important tool of the model - a least-cost path tool which outputs a polyline feature class that holds the least cost paths. The least cost paths are delineated from the spill points, or source locations, to the destination points which are the access points embedded in the cost backlink raster. An example of a portion of the output polyline feature class of least-cost paths is displayed in Figure 44. 


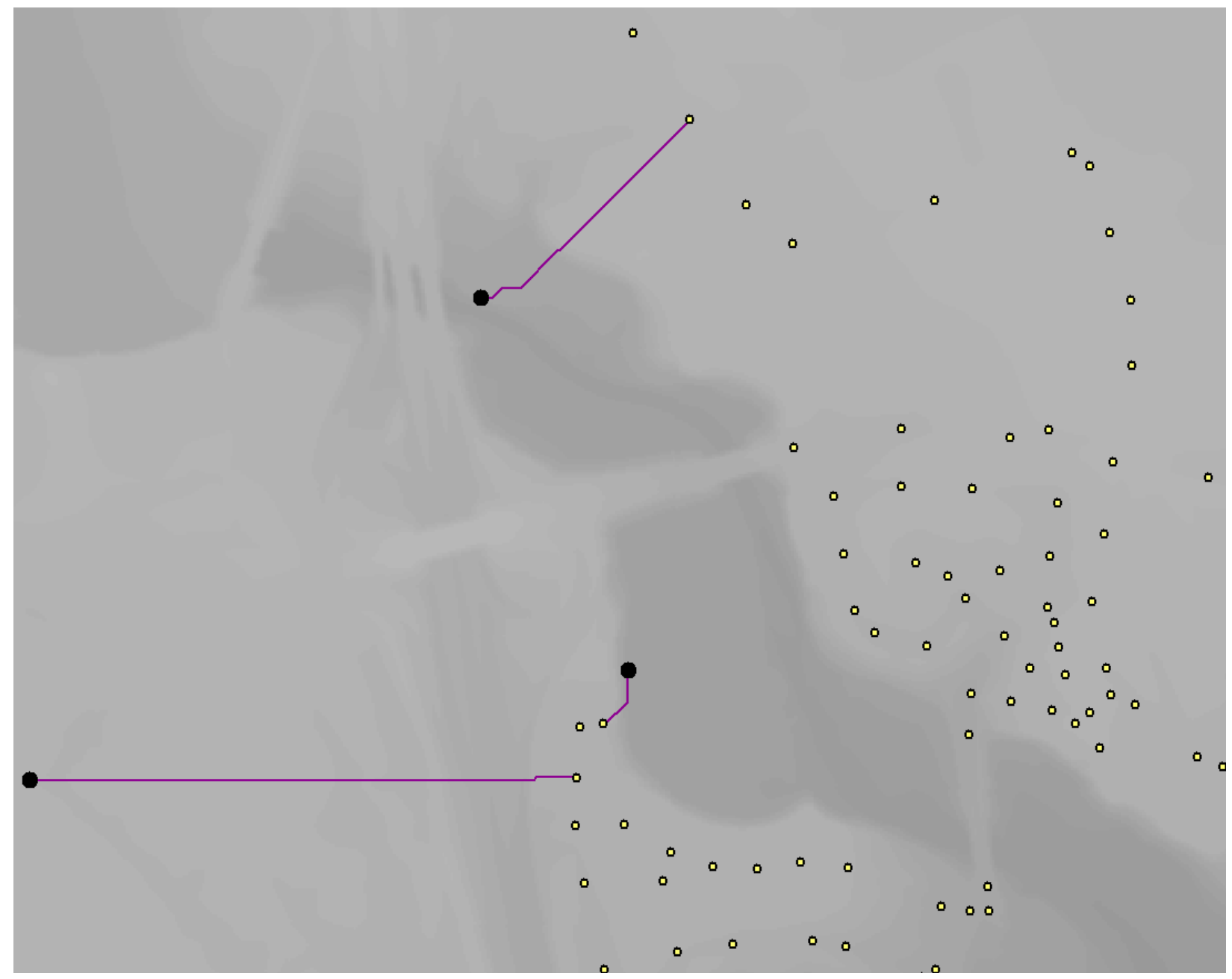

Figure 44. Examples of least-cost paths of the output polyline feature class created.

The larger points represent spill points and the smaller points represent the destination access points. The line connecting the two points is the least-cost path from the oil spill point to the nearest access point of the storm sewer system. A closer look at the spill paths reveals that the first path from the bottom of Figure 44 connects the spill and destination points exactly as highlighted next in Figure 45. 


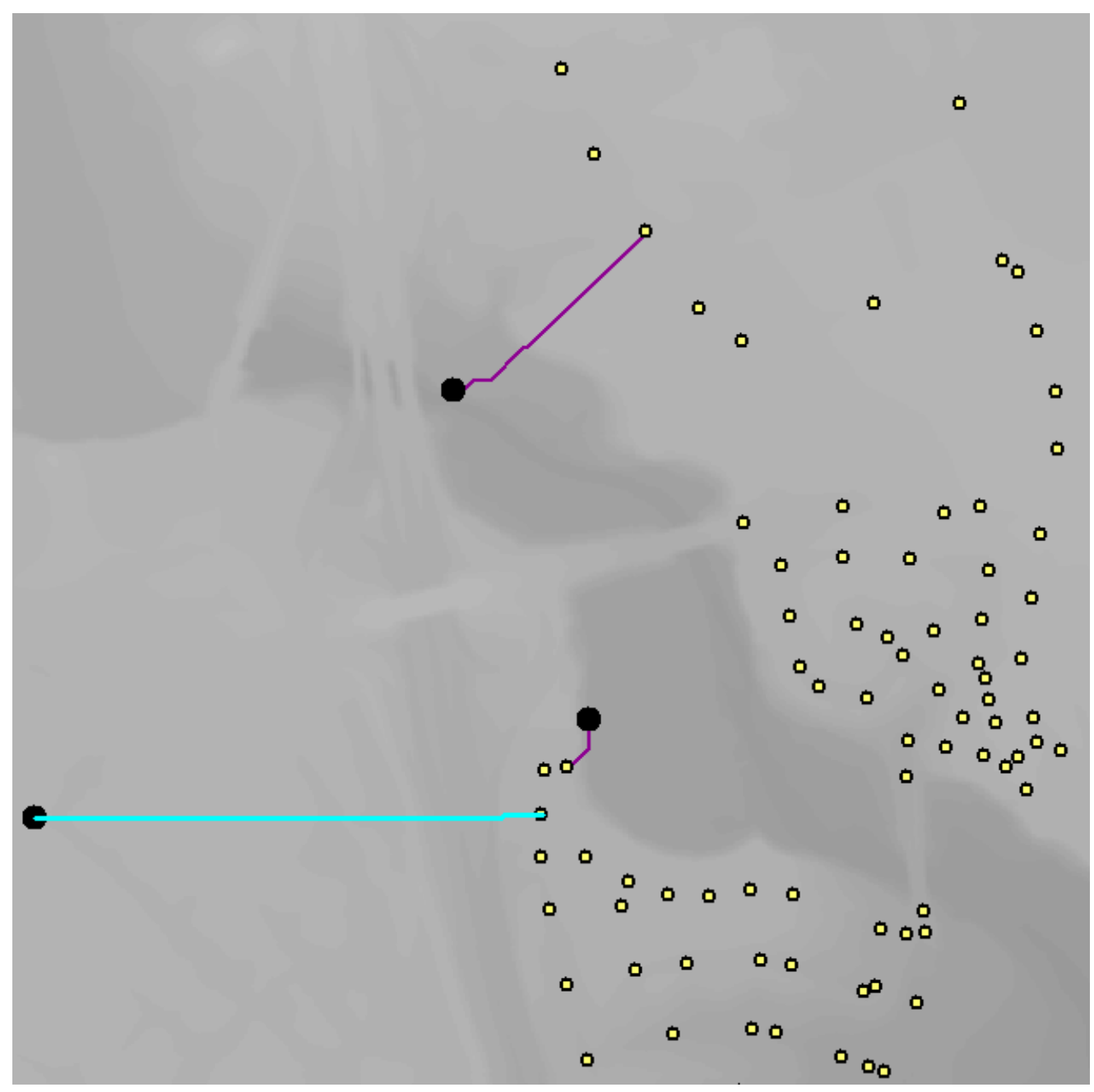

Figure 45. The highlighted spill path contacts both points to connect them.

Most of the spill paths do not contact the beginning or ending points exactly but approximate a path between both points as illustrated in Figures 46 and 47.

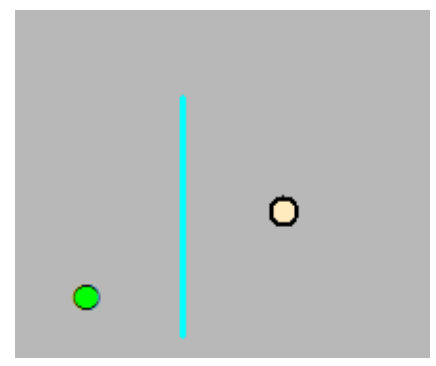

Figure 46. A short spill path does that does not connect the spill point (green) to the access point (beige). 
The length of the path shown in Figure 46 is 3 metres which is the width and height of each grid cell. The resolution of the raster data sets appears to have an impact on path lengths which are of the same or similar size. The error shown in Figure 46 is a rarity as most paths more accurately approximate the distance between the two points as demonstrated in Figure 47.

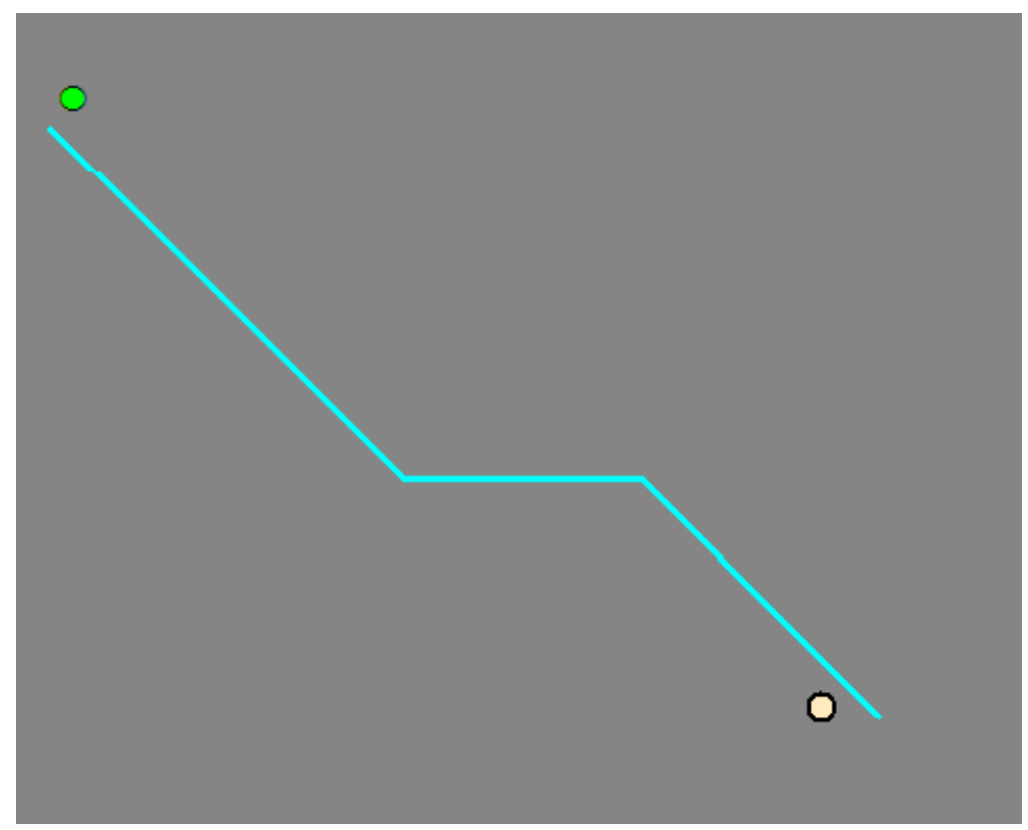

Figure 47. A typical line in the output polyline feature class that approximates the least-cost path between the spill point (green) and the access point (beige).

The least-cost path line illustrated in Figure 47 demonstrates a typical and acceptable result despite the apparent error caused by the lack of contact between the line and the beginning and ending points. The backdrop in Figures $44-47$ is the DEM as opposed to the cost backlink raster that supplements the delineation of the least-cost pathways. The DEM is used to see the paths more clearly. 
The next 13 geoprocessing tools in the model modify the given attribute table output of the polyline feature class of spill paths to calculate the travel-time, in seconds, taken by the spill to travel along the least-cost path to the nearest access point which is the destination location. The next part of the model containing the loop retrieves the minimum spill travel-time, in seconds, and replaces any non-numeric invalid entries with this minimum spill travel-time (s). A minimum travel-time of 28.85 seconds was used to replace any text entries which were the result of equal elevation values for the both the beginning and ending points of the least-cost path line.

Another column is added by the model to the attribute table to store spill travel-time values in minutes. The travel-time in minutes was then calculated to yield an average travel-time of 3.08 minutes. The minimum and maximum travel-times (min) are 0.48 minutes and 37.72 minutes respectively. The results are in agreement with the assumption of 5 or 10 minutes and a priori knowledge derived from experience. Some of the results do not fall exactly within the range given by the assumption, which is loosely applied, yet still acceptable because they are realistic and logical.

The final part of the model entails the development and implementation of a geometric network within the study area. A portion of the geometric is illustrated in Figure 48 against the backdrop of the DEM. 


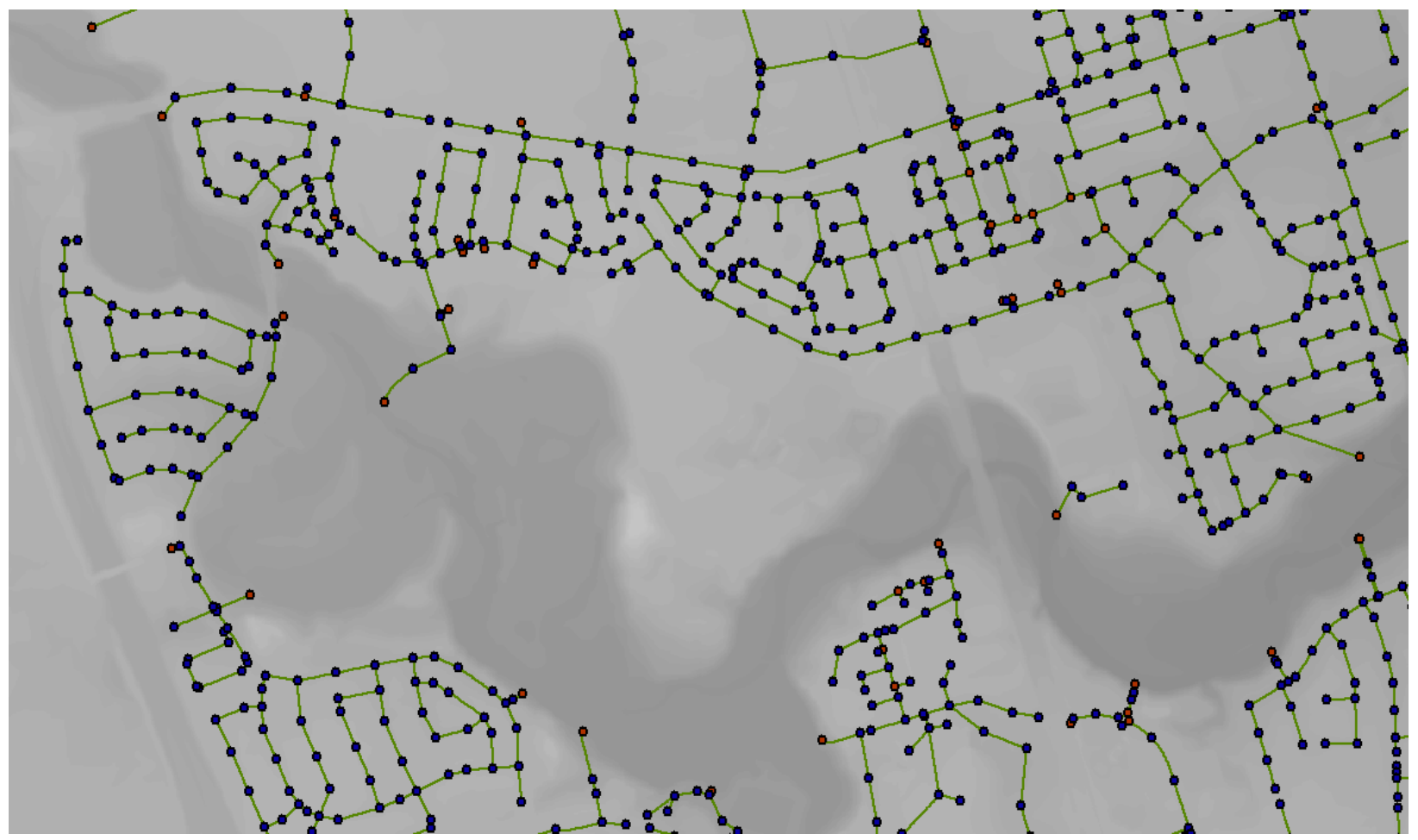

Figure 48. A portion of the geometric network developed and used in the spill path and travel-time model.

Figure 48 illustrates the connectivity of the sewer lines (green) and the access points (blue) that comprise the geometric network. The underlying DEM illustrates a sizable waterway or river that separates the two sides of the geometric network in a certain area. The red access points are extraneous points added by the geoprocessing tools as a distinct shapefile of junction points of the geometric network. The additional shapefile represents access points, which are junction points, lying at the ends of sewer lines that did not initially have access points. These additional points can be removed since they are not a necessary part of the geometric network. Figure 49 depicts the geometric network without the additional junction end points. 


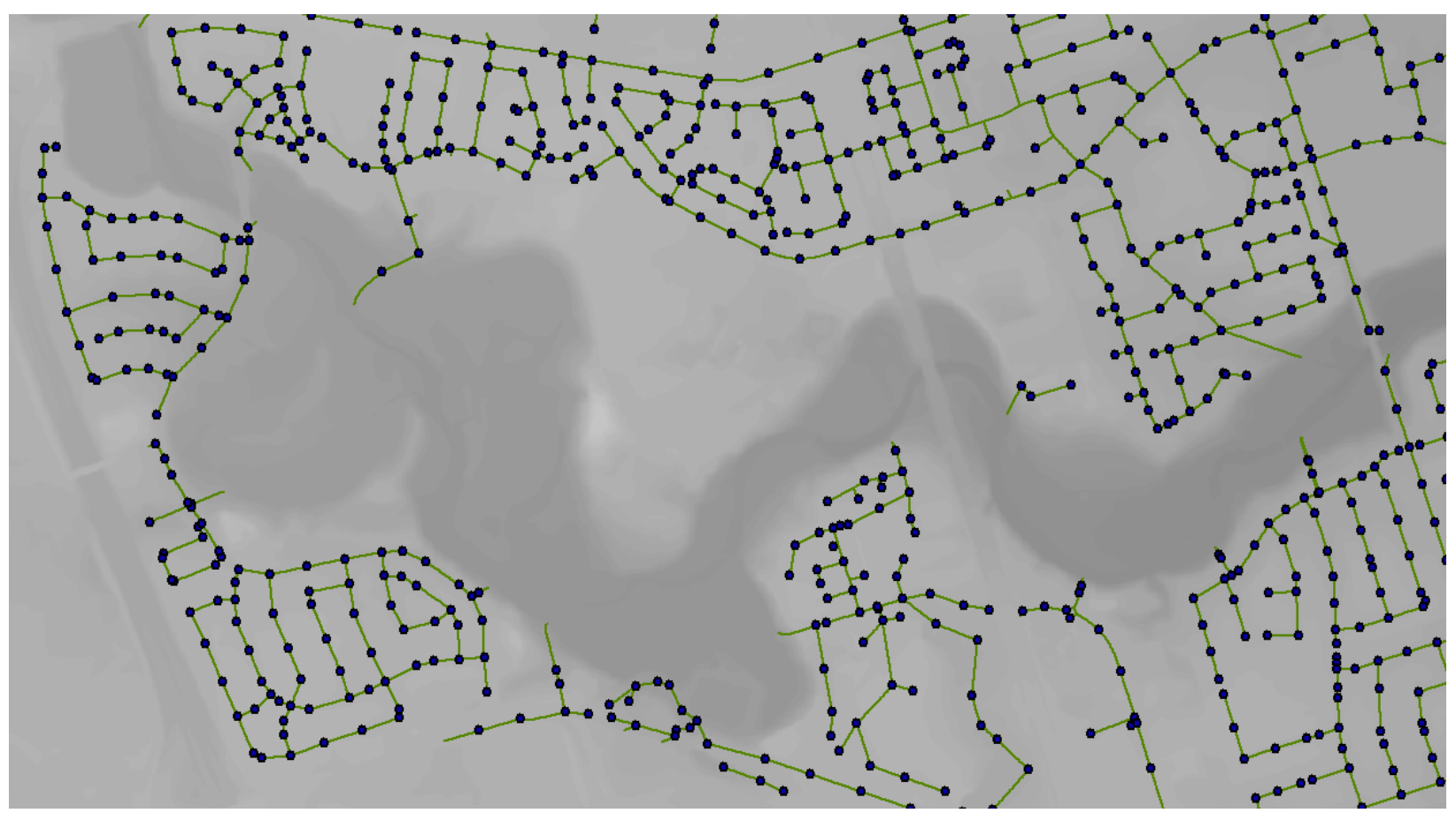

Figure 49. Geometric network without additional junction end points.

The additional junction end points are automatically inserted at the end of sewer lines to establish connectivity between lines/edges and points/junctions. The junction points are considered as build errors as listed in a corresponding attribute table of 2,169 records. By the standards of geometric network development there are 2,169 errors, however the additional points are simply a part of a supplementary shapefile of access points that are not a necessary component of the model. The data set within the geometric network developed is therefore comprised of three data layers: (1) 15,970 access points into the sewer system, (2) 17,069 storm sewer lines, and (3) 2,169 additional access points as storm sewer line end points.

The final task of the model is to trace the spill pathway through the geometric network representing the storm sewer system and associated access points. The end points of the 
least-cost paths are converted into a shapefile of points (flags) that signify (or flag) the beginning point of the pathway through the storm sewer system. In this case, a shapefile of 37 points or flags is created to mark the starting points of the spill pathways through the storm sewer system. The points are connected to the access points that they flag to represent the beginning points of the spill paths.

Before tracing the spill through the storm sewer system, the flow direction was set to trace all downstream paths of the spill since other options, such as tracing upstream paths, are available. The flow pathways through the storm sewer line channels were then traced throughout the geometric network for each of the 37 starting points which were flagged. The resulting data for each of the three data layers of the geometric network data set are then extracted into three separate data sets and developed into three distinct files. This collection of output files with features involved in the tracing of the spill paths is displayed in Figure 50. The bits of orange represent storm sewer lines, the light blue dots represent access points and the beige dots represent the supplementary access points which can be included, but are not required for the development of the model. Each of the output files resulting from the tracing of spill pathways through the sewer system is difficult to discern, therefore the illustration in the figure is given to provide an overview of the output. 


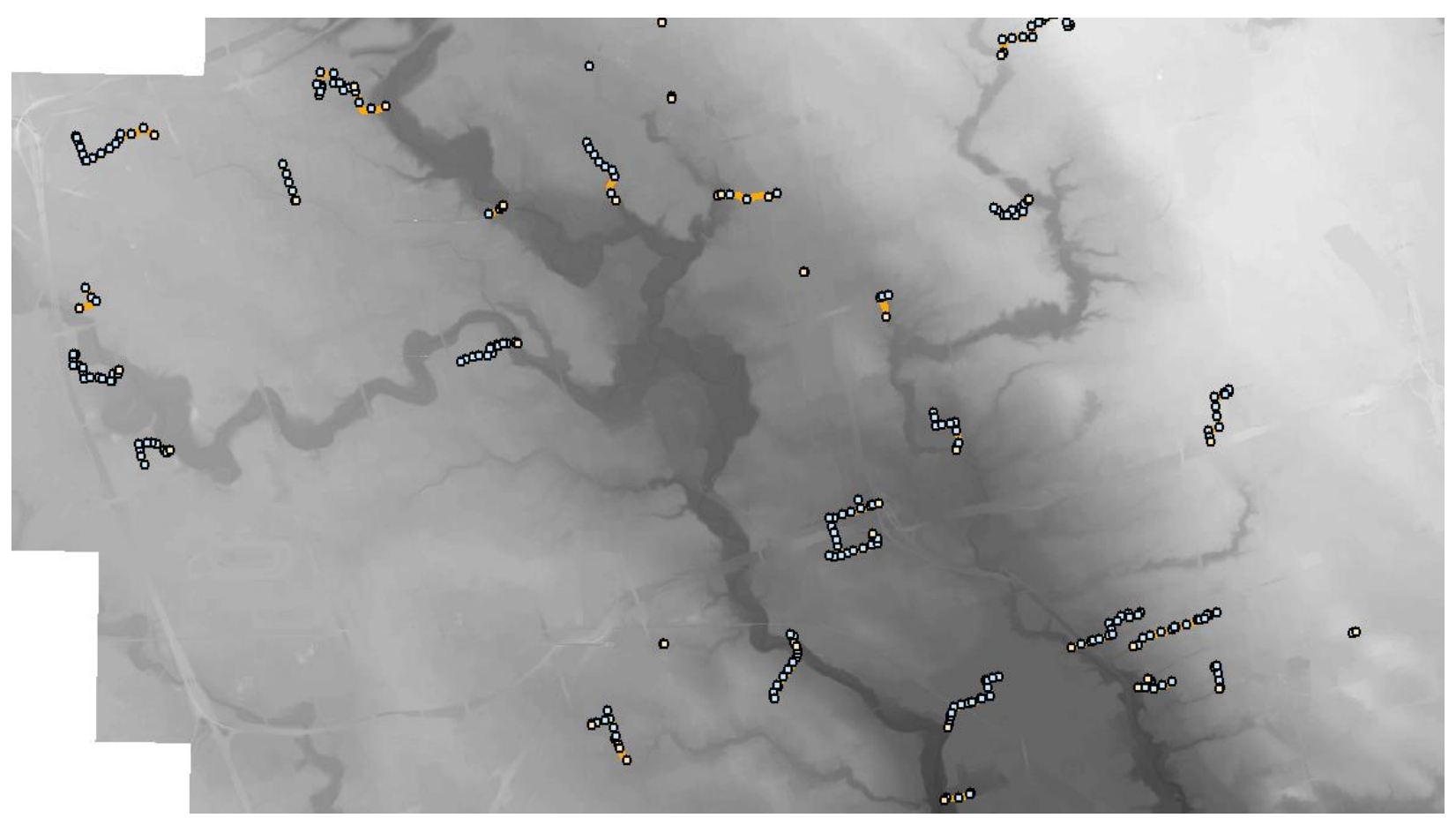

Figure 50. Three output files resulting from tracing spill pathways through the storm sewer system.

The storm sewer lines involved in the trace were derived from the sewer lines shapefile of the geometric network and are still composed of storm sewer line segments. All storm sewer line segments within the study area are associated with travel-time (min) values in the corresponding attribute table. Since the pre-calculated travel-time (min) values are still attached to each sewer line segment, a final geoprocessing tool was used at the end of the sequence of tools within the model to sum travel-time values for line segments within the same spill path. Storm sewer line segments with coincident end points were merged as well as the common attribute field of pre-calculated travel-time (min) values. The intent was to create a single pathway for each spill through the storm sewer system. However, since all sewer line segments are not coincident and some coincident sewer system spill pathways exist, only some line segments were merged. Some line segments 
were merged while others were not to yield a segmented yet continuous pathway for each spill.

Nonetheless, the travel-time ( $\mathrm{min}$ ) values for each storm sewer line segment or merged series of segments can be added and then combined with the associated travel-time (min) values for overland flow from the earlier output attribute table. The final result is a delineated overland and storm sewer line path with a total travel-time (min) value. The overall result is illustrated in Figure 51 against a blank backdrop.

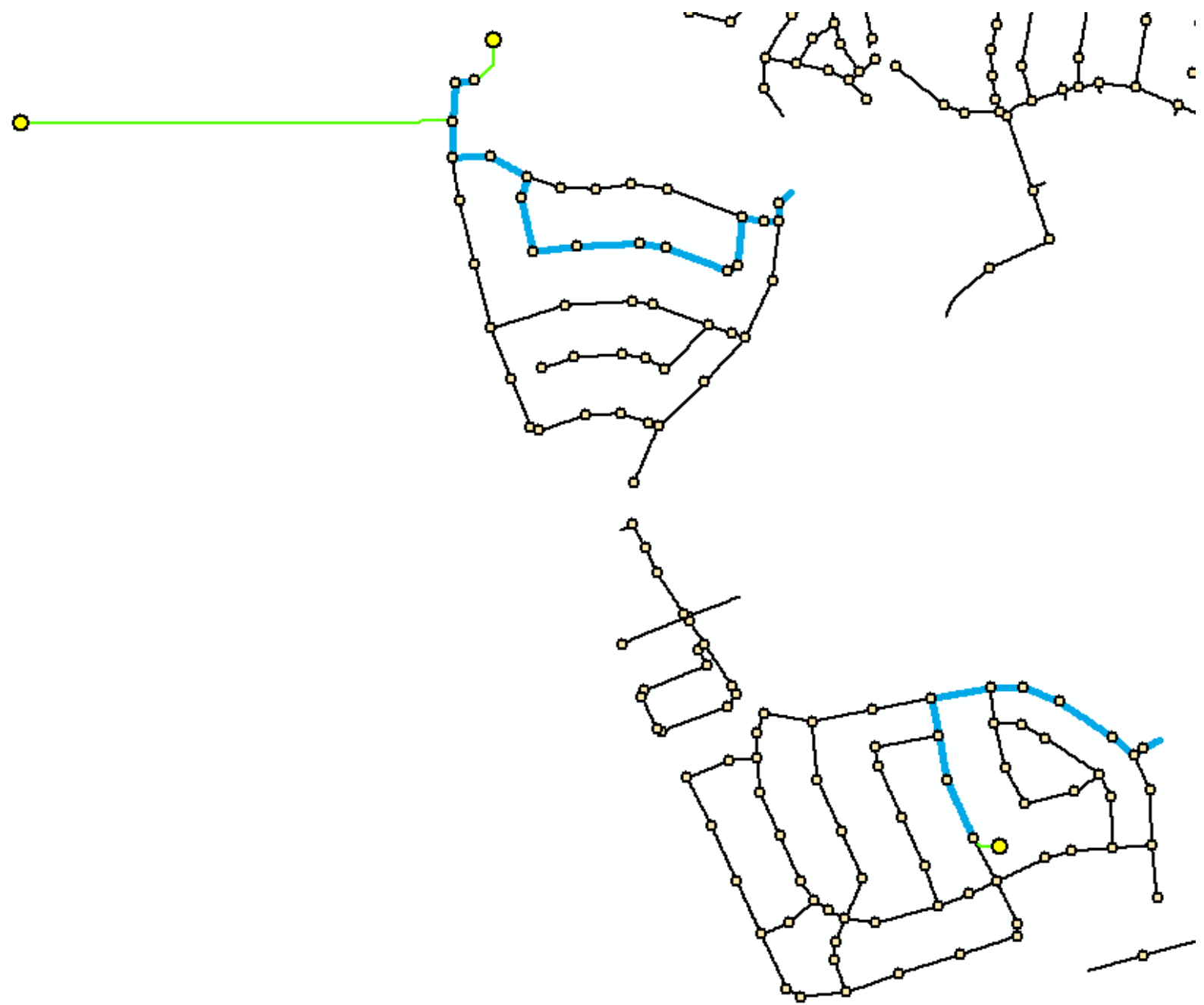

Figure 51. A section of an example of the final result of the spill path and travel-time model. 
The yellow points in Figure 51 are spill points that are connected to the access points (beige) by an overland spill path line (neon green). The connected blue sewer line segments are the portions of the storm sewer line system that were traced to form a spill path through the storm sewer system. The travel-time ( $\mathrm{min})$ values for the overland flow can be added to the travel-time (min) values of the adjoining sewer line segments to yield a final, overall travel-time (min). This final travel-time value can be used in spill response measures to more accurately calculate the amount of time required to reach the spill area on-land or in the storm sewer system to possibly divert or stop the spill.

The two spill points at the top of the figure share a common spill pathway for part of the journey through the storm sewer system. If all of the connecting line segments were merged to form a single pathway, it would result in an inaccurate portrayal of the spill pathway for the uppermost point since its pathway is not entirely the same as the pathway of the other spill point. Furthermore, the travel-time cannot be accurately summed for spills sharing a common pathway or part of a common pathway since sewer pipe segment travel-times are pre-calculated. Therefore, even if the endpoints of sewer line segments are made coincident, a more accurate travel-time (min) will not be achieved. 


\section{Chapter 5: Application of Stationary and Transportation-Related Spill}

\section{Models to the Spill Management Planning Framework}

The development of stationary and transportation-related spill models was discussed in the previous chapter. Each type of spill model was then applied to each of the three stages of prevention, control, and response and clean-up to develop planning tools or planning models for each stage of the spill management framework. The planning tools or planning models developed for each of the three stages is shown in the boxes of Figure 7 and discussed next for stationary and mobile or transportation-related spills.

\subsection{Development of Planning Tools for the Stationary Spill Model}

\subsubsection{Stationary Spill Model Planning Tools for Spill Prevention}

In Chapter 4, basic results stemming from preliminary analyses of the initial overall main spill database encompassing all spills, both stationary and transportation-related, were discussed. Prevention planning tools based on the data set for all inland oil spills in Ontario were first developed. For instance, the majority of inland oil spills in Ontario occur as a result of leaks and breaks of containment systems and pipe or hose leaks. Containment systems generally refer to fixed structures such as oil tanks while pipe or hose leaks refer to passageways for transporting substances which may sometimes include tubing. A general prevention planning tool based on this finding is improved maintenance, inspection and replacement of oil containment systems and pipes, hoses and tubing. 
Since most inland oil spill events in Ontario can be explained by equipment failure or by equipment/vehicular-related reasons and analytical or human error, improved equipment maintenance and training of operator personnel could be implemented as a prevention planning tool for all spills. Equipment should be inspected at regular intervals and operator knowledge and skill tested regularly.

A similar approach was taken to develop planning tools for the stationary spill sub-database of gasoline spills for the case study area. The bivariate histogram of reason versus cause in Figure 25, illustrates the relationship between the leak/failure/overflow of machinery, equipment, containers or tanks and negligence/operator error.

Negligence/operator error are related to the efficacy of staff and managers with respect to performing duties. Therefore, a preventative planning tool for the case study area is improved training and staff education as well as more effective oversight by managerial authorities. Based on the results, if staff attend to machinery, equipment, containers or tanks more frequently and thoroughly, and operate such devices with less error, a reduction in on-land oil spills should be evident.

An enhancement of preventative maintenance practices and improved operator training and awareness of spill potential could also be implemented to better maintain and prolong the life cycle of machinery or equipment. On the contrary, pipe leaks or failures in the case study area are mainly due to material damage or failure as opposed to operator error or negligence. Therefore, more durable pipes which are made of materials that are less likely to deteriorate and leak could be installed or used to replace existing, more fragile 
pipes as a preventative planning tool. This recommendation is supported by the data illustrated in the bivariate histogram of Figure 25.

As previously discussed, the bivariate histogram of Figure 26 illustrates how the bulk of gasoline spills occurring in the Humber River Watershed originate in the petroleum sector due to pipe/hose/valve/fitting leaks or failures and machinery, equipment, container or tank leak/failure/overflow issues. Petroleum sector companies within the Humber Watershed can be informed of the inherent weaknesses of their industrial framework to better target areas in need of improvement.

Higher quality mechanisms and devices could replace existing ones and greater emphasis could be placed on the immediate reporting of any noticeable malfunctioning of devices or system elements. Based on Figure 26, the petroleum sector within the case study area should be the focus for preventative strategies while industries with few or no spills, such as the service industry, do not require as much attention.

\subsubsection{Stationary Spill Model Planning Tools for Spill Control}

The second box listing control planning tools in Figure 7 entails a spill location analysis to identify areas for control measures. Examples of control measures are treatment devices that include treatment/flow control Best Management Practices (BMPs). Treatment/flow control BMPs are structural facilities that are designed to remove pollutants such as oil from stormwater runoff prior to discharge into receiving waters and/or to reduce or control runoff volumes (Transportation Research Board [TRB], 
2012). Common examples include detention basins (e.g. stormwater management ponds), infiltration systems, filter strips, roadside swales, and sumped catch basins (TRB, 2012).

The typical design of an urban stormwater-collection system is shown in Figure 52 and entails routing surface runoff from streets to pipelines through storm sewers (Chin, 2013).

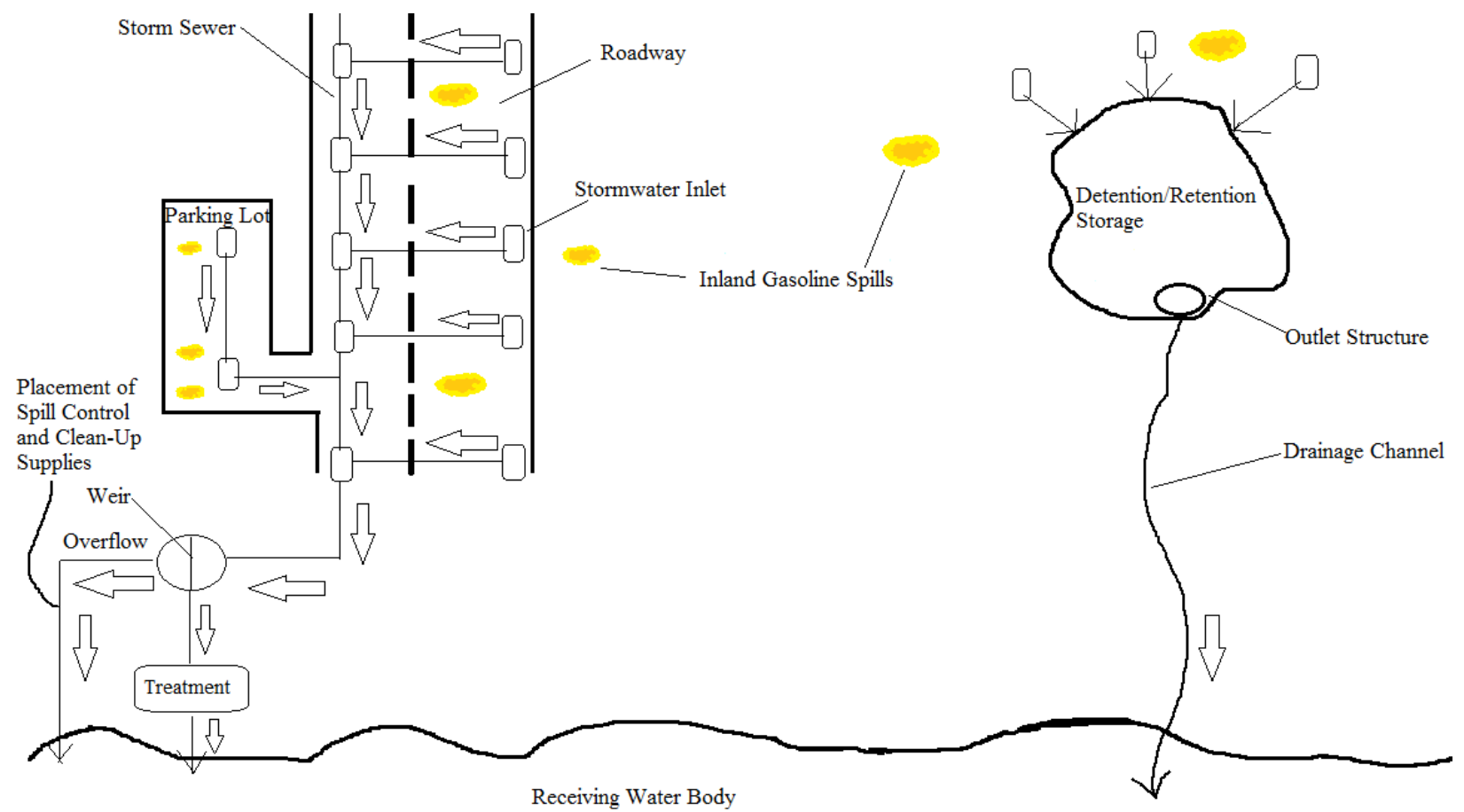

Figure 52. A typical design of a stormwater-collection system with gasoline spills. (Source: Adapted from Chin, 2013)

Figure 52 illustrates how urban runoff enters the stormwater collection system through street gutters and inlets that are connected to storm sewers by inlet structures such as catch basins. The collected runoff is transported in the storm-sewer system to a treatment unit such as a detention basin or an infiltration basin. Effluent from the treatment unit is then transported and released into a receiving water body such as a river or a lake (Chin, 
2013). Any excess runoff collected usually bypasses the treatment unit through a weir-type structure and is released directly into the receiving water body without treatment (Chin, 2013). Urban runoff from other types of surfaces other than roadways and parking lots can also be collected by inlet structures and directed to a treatment unit before being released into a receiving waterway, as illustrated on the right-hand side of Figure 52 (Chin, 2013).

Figure 52 also illustrates a typical scenario of a spill prone area in the Humber River Watershed to demonstrate the routing of gasoline spills for both recorded and predicted spills (yellow and orange spots) in the case study area. On the left-hand side of Figure 52, a few gasoline spills have occurred on the parking lot and on the roadway while on the right-hand side, gasoline spills have occurred in other kinds of areas such as on the property of an industrial plant. In both cases, the gasoline spill is treated before entering the main receiving water body of the case study area, the Humber River.

If the amount of spilled gasoline is high, then first responders should anticipate an excess amount of gasoline flowing into the Humber River or water body without being treated. They should position themselves at an appropriate location as indicated on Figure 52 (see "Placement of Spill Control and Clean-Up Supplies") to intercept the spill from entering the river by using booms and other spill control devices. The path taken by the spill, both overland and in the storm sewer system, can be predicted by the GIS-based spill path and travel-time model developed. Travel-time analysis for spill control strategies aims to prevent excess, untreated spilled oil from entering water bodies within close proximity. 
Another objective is to reduce or control the volume of spilled gasoline from entering the water body using spill control equipment.

In a case study, a stormwater management pond or stormwater detention pond at a public school was retrofitted to treat the "first flush" of surface runoff from the parking lot (McDuffie et al., 2015). First flush is the phenomena that the highest pollutant concentrations and loads occur in the first portions of the runoff hydrograph or in the initial stages of a rain event (TRB, 2012). The detention/retention storage unit of Figure 52, representing a potential or existing stormwater management pond, can be retrofitted, if required, to treat gasoline and other oil spills in advance of their discharge into the river. An oil-water separator can be attached to the outlet structure to receive any incoming oil spills which will be combined with water from the pond.

If the inlet structures connected to the inlets shown in Figure 52 are assumed to be catch basins, the catch basins can be retrofitted by installing catch basin inserts, an example of which is shown in Figure 43 which is a sketch of a photograph of a catch basin insert.

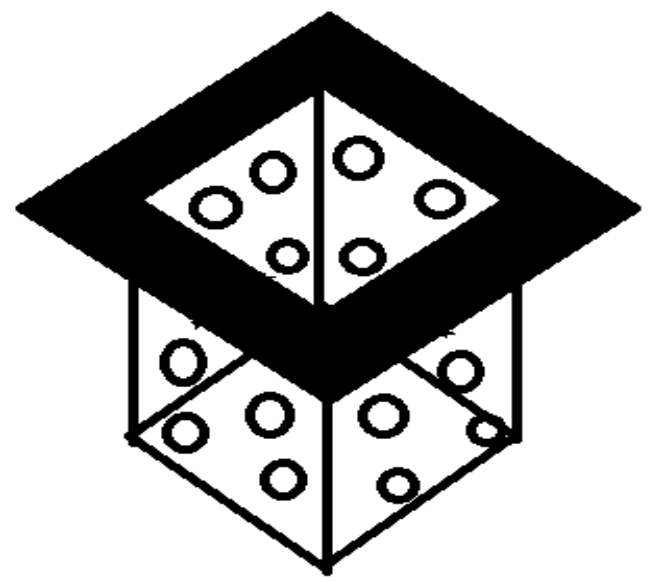

Figure 53. A sketch of an example of a catch basin insert to target oil and grease.

(Source: Adapted from TRB, 2012) 
Catch basin inserts are typically designed for easy drop-in installation in existing catch basins and some inserts include oil-adsorbent media to target the removal of oil and grease (TRB, 2012).

Oil-water separators that trap floatables such as free oil and grease can be installed as the treatment unit or connected to the outlet structure of the detention/retention storage as shown in Figure 52. Oil-water or oil-grit separators are chambered tanks, as shown in Figure 54, which are designed to remove gross pollutants and solids by sedimentation and to specifically trap free-phase oils and grease (TRB, 2012).

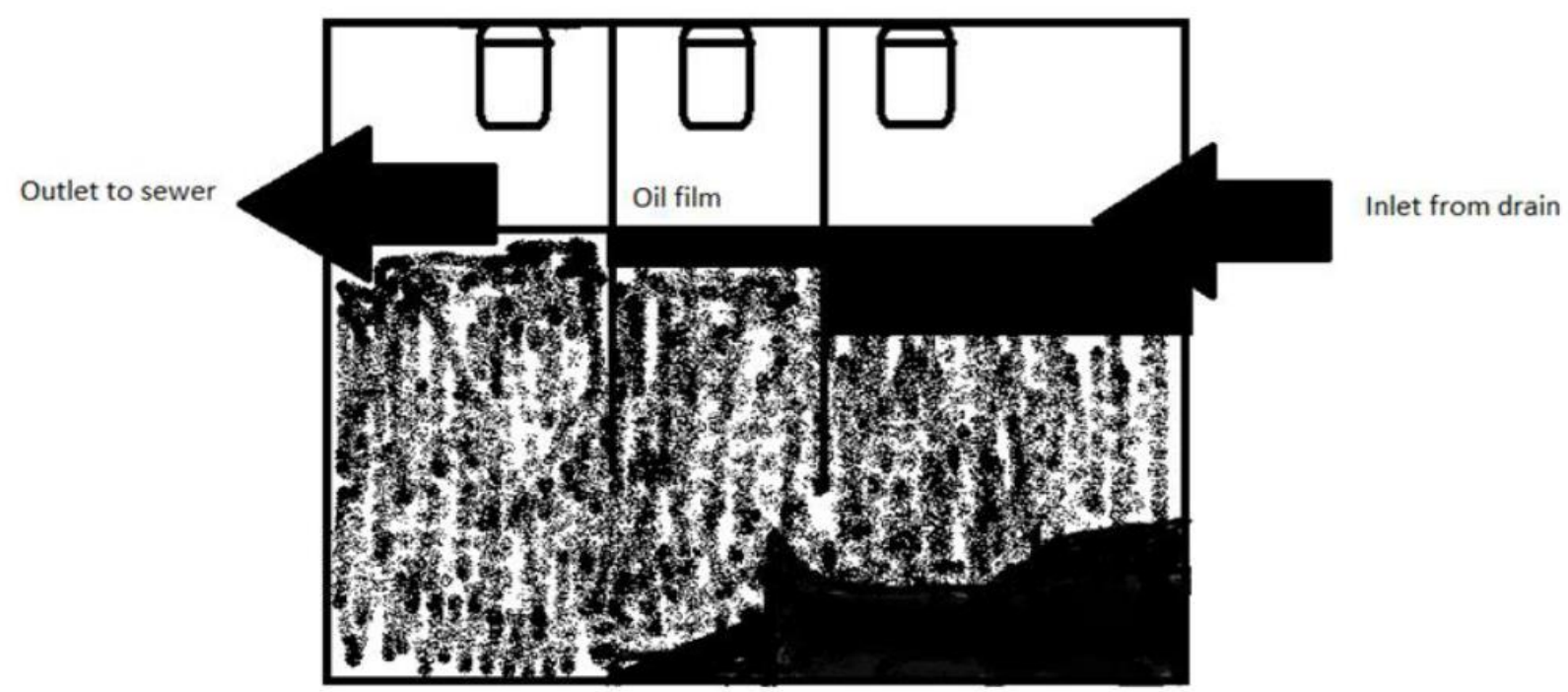

Figure 54. A sketch of an oil-water separator used to trap free-phase oils and grease. (Source: Adapted from TRB, 2012)

Oil-water separator capacities come in a range of sizes from 50 to 20,000 gallons and should be sized according to the spill volume characteristics of spills expected to occur in spill prone locations which is a spill control planning tool. Spatial prediction is first 
performed using the macro program to predict expected spill occurrences in the study area based on historical spill data of the same area. The historical spill data set may contain inaccurate or missing spatial data to correctly describe the location of past spill events that may hinder the ability to more accurately predict future spill events. Since predicted data is based on historical data, predicted future spill locations reflect the general area of past spill locations. However, some predicted future spill events may occasionally be situated in unusual locations in which a future spill event is unlikely to occur such as in the middle of a park.

The predicted spill locations from the aggregate output can be plotted and areas of high spill concentrations can be determined using hot spot analysis to identify spill prone locations. Control/response options can then be placed in these spill prone locations. For instance, the results from the aggregate simulation output data state a predicted mean value of approximately $81 \mathrm{~kg}$ or $112.5 \mathrm{~L}$ in Table 9 . The capacity of oil-water separators installed in the case study area should reflect the average predicted spill volume for the long-term planning horizon at minimum.

Oil-water separators require regular cleaning of solids and organics that have accumulated. A vactor truck is used to perform regular cleaning in order to ensure design-level performance and to minimize washout. The maintenance required for catch basin inserts is generally minimal and is mainly focused on cleaning and disposing of accumulated solids. Frequent inspection may be required to assess maintenance needs or 
to assess clogging and potential safety hazards which could result in very high costs, potentially rendering this option as infeasible (TRB, 2012).

Surface detention facilities such as stormwater management ponds have the advantage of only requiring fairly routine and infrequent maintenance activities (TRB, 2012).

Municipal staff and other appropriate personnel must be thoroughly trained in maintaining and inspecting treatment/flow control BMPs for on-land oil spills to ensure their proper functioning.

A siting analysis for placement of BMPs and treatment units to target oils and grease is a control planning tool that should be used in conjunction with highly trained personnel who can skillfully control and mitigate the spreading of future on-land oil spills. Oil tanks and other containment devices such as containment walls can be designed with a greater emphasis on safety to help control the spreading of a spill. Containment walls are usually placed around oil tanks and can be sized based on predicted spill characteristics of the facility and tank capacity to capture potential leaks or spills. Frequent or non-stop monitoring of oil-related systems and infrastructure, as currently performed by major oil pipeline companies, also helps to control on-land oil spills.

\subsubsection{Stationary Spill Model Planning Tools for Spill Response and Clean-Up}

A GIS-based spill path and travel-time model was developed that can be applied to both stationary and mobile spills. The model determines the approximate amount of time required for the spill to travel overland to the nearest access point of the storm sewer 
system and through the storm sewer system to the outlet structure or exit point. The delineated spill path and estimated time taken by the spill to traverse the path has been applied to develop the planning tools in the third box shown in Figure 7. The third box addresses Response and Remedial Action that entails travel-time analysis for spill response and clean-up strategies.

For travel-time analysis, suitable spill response and clean-up strategies for each spill are based on the estimated total (overland and sewer system) or partial (either overland or sewer system) travel-times (minutes or seconds) as determined by the model. An estimated total spill travel-time of 15 minutes, for example, allows first responders approximately 15 minutes to travel to the exit point of the storm sewer system which was predicted by the model to prevent the spill from entering the water body. An estimated partial overland spill travel-time of 5 minutes, for example, allows first responders approximately 5 minutes to travel to the predicted access point of the storm sewer system to prevent the spill from entering the pipeline system.

The spill destination points of either the sewer system access or exit points are predicted by the spill path and travel-time model. The model therefore determines where the spill can potentially be intercepted and how long the spill will take to reach that point. The time taken to reach the destination point can be used by spill response personnel to gather equipment and outline a route to reach the destination point as quickly as possible.

Figure 51 illustrates the overland spill paths connecting predicted spill points to the corresponding least-cost sewer access points. Based on the beginning least-cost sewer 
access points, the sewer system pathway is traced to the exit point. The spill can potentially be intercepted at any point along the overland path or at the exit point of the storm sewer system path. Therefore, response personnel can plan in advance where they intend to intercept the spill.

Regardless of whether the spill destination point is an entrance or exit point of the storm sewer line system, an estimated or assumed travel-time of 5 to 10 minutes is usually not enough time for a spill response and clean-up crew to reach the predicted destination of the spill. Therefore, other planning tools must be developed to facilitate the response and clean-up mission.

An example of such a planning tool is shown in the box of Figure 7 for Response and Remedial Action as spill location analysis for the placement of spill clean-up kits and other spill response and clean-up equipment. Spill clean-up kits and spill response and clean-up equipment can be placed at strategic locations or close to areas with frequent spills to facilitate the clean-up process.

Figure 24 illustrates a simulation data set of predicted spills for the lower region of the Humber River Watershed within the City of Toronto. It also illustrates a few clusters or groups of a few spills in various locations. Municipal works yards located near these groups or clusters can be stocked with spill clean-up supplies and equipment. Once response and clean-up personnel arrive near the spill site, they will be able to quickly access and utilize the supplies and equipment. These storage centres can also serve as a source of additional or back-up spill clean-up supplies and equipment. 
The supplies and equipment can be stored in more strategic locations by tailoring the stored supplies and equipment of each municipal works yard to similar characteristics of certain groups of spills. For example, the results in Figure 51 can be used to place supplies and equipment for spill clean-up in municipal works yards which are not only close to the colour-coded or clustered groups of spills but are also specific to spills with characteristics common to the group.

If a certain portion of the sewer line system experiences repeated spills, then spill response and clean-up equipment can be stored near the sewer line segment. For example, the upper part of Figure 51 illustrates how two inland oil spills beginning at distinct starting points share a common portion of the sewer line pathway. Since this series of sewer line segments is subject to frequent spills, spill equipment and supplies could be stored at a convenient location close to the sewer line path. Figure 51 has been modified to Figure 55 in order to illustrate the placement of spill equipment and supplies close to the exit point of both spills to prevent the spill from entering the water. A small municipal storage centre for spill equipment and supplies is represented by the yellow pentagon symbol. More information about the placement site is required, however, because there may be roads or other buildings which prevent the placement of the equipment and supplies. 

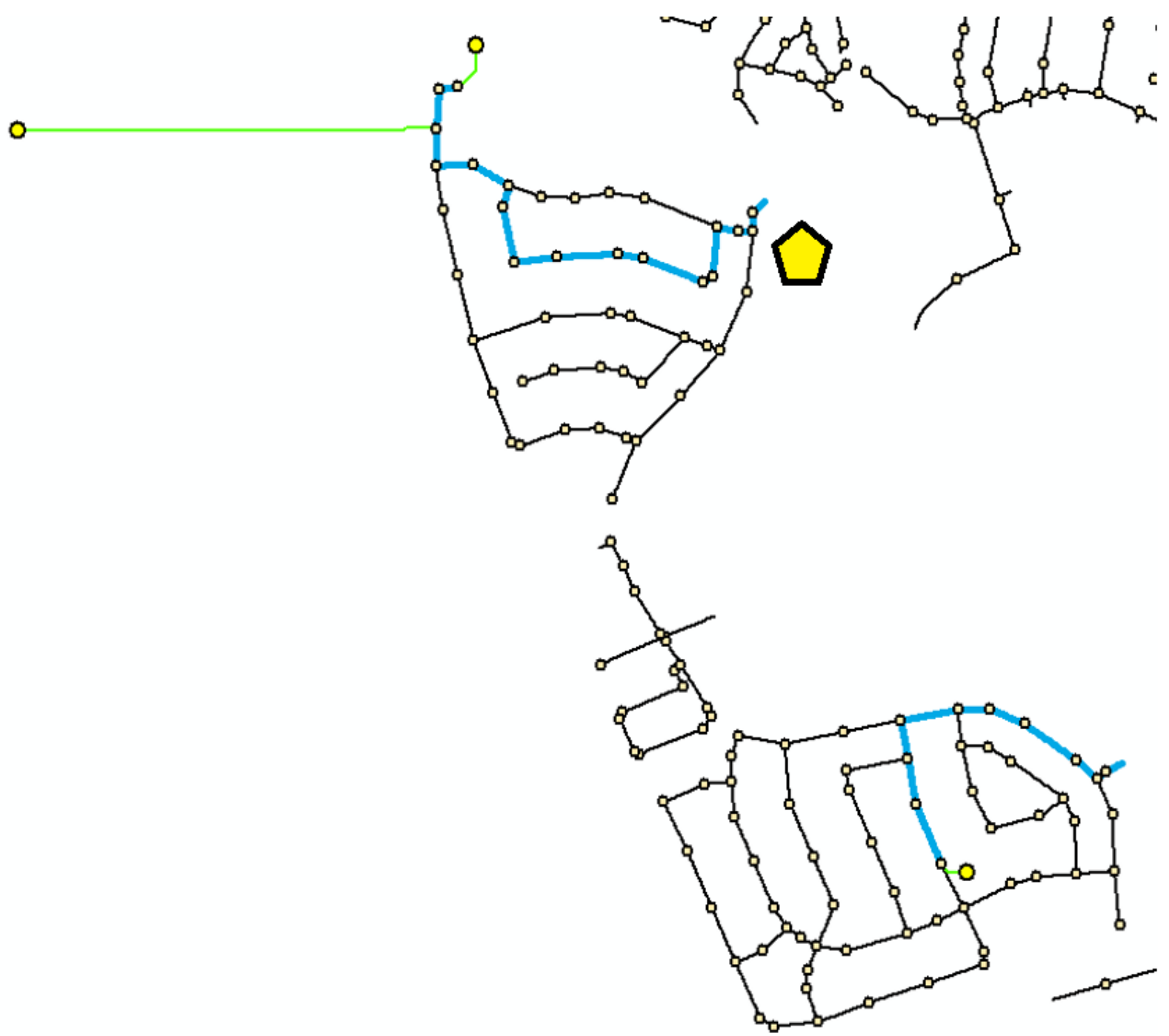

Figure 55. Storage of spill clean-up supplies and equipment near a spill exit point.

\subsection{Development of Planning Tools for the Transportation-Related Spill $\underline{\text { Model }}$}

\subsubsection{Transportation-Related Model Planning Tools for Spill Prevention}

The investigation of transportation-related or mobile inland spills occurring within 50 metres of major Toronto highways revealed that approximately three-quarters of all 
highway spills occur at or on interchanges or close to interchanges. Since the majority of oil spills occurring on highways occur at interchanges, a planning tool to enhance the safety of interchange design was developed as a preventative strategy. However, reliable, specific and explicit quantitative correlations between interchange parameters and safety are not available (TAC, 2007). As a result, the design domain concept for interchanges is applied in terms of empirical information such as "best practices" (TAC, 2007).

Although some empirical information has been obtained by displaying and analyzing historical data for mobile highway-related oil spills, the actual location information is often too vague or inaccurate to draw specific conclusions as to the modification of highway design features. The data layer symbolizing major Toronto highways also does not include explicit details regarding highway geometric design features.

Despite the lack of detail regarding historical spill location data and highway feature attribute data, some general recommendations can be made as prevention planning tools to enhance the safety of interchanges through design to help prevent future spills. Interchanges present a challenge to drivers by requiring them to make a complex set of decisions which necessitate quick evaluation and action (TAC, 2007). Driver stress can be reduced at interchanges by keeping the alignment simple and direct, providing sight distances greater than the minimum stopping sight distances, and maintaining design consistency (TAC, 2007). Using above-minimum design criteria for other geometric elements can also help to alleviate driver stress (TAC, 2007). Increases in traffic volume and decreases in curve radius often lead to an increase in collisions on ramps and 
connecting roads (TAC, 2007). Figure 56 illustrates a curve radius of $36 \mathrm{~m}$ at an interchange ramp. A decrease in this curve radius will create a sharper turn on the ramp that may lead to more collisions.

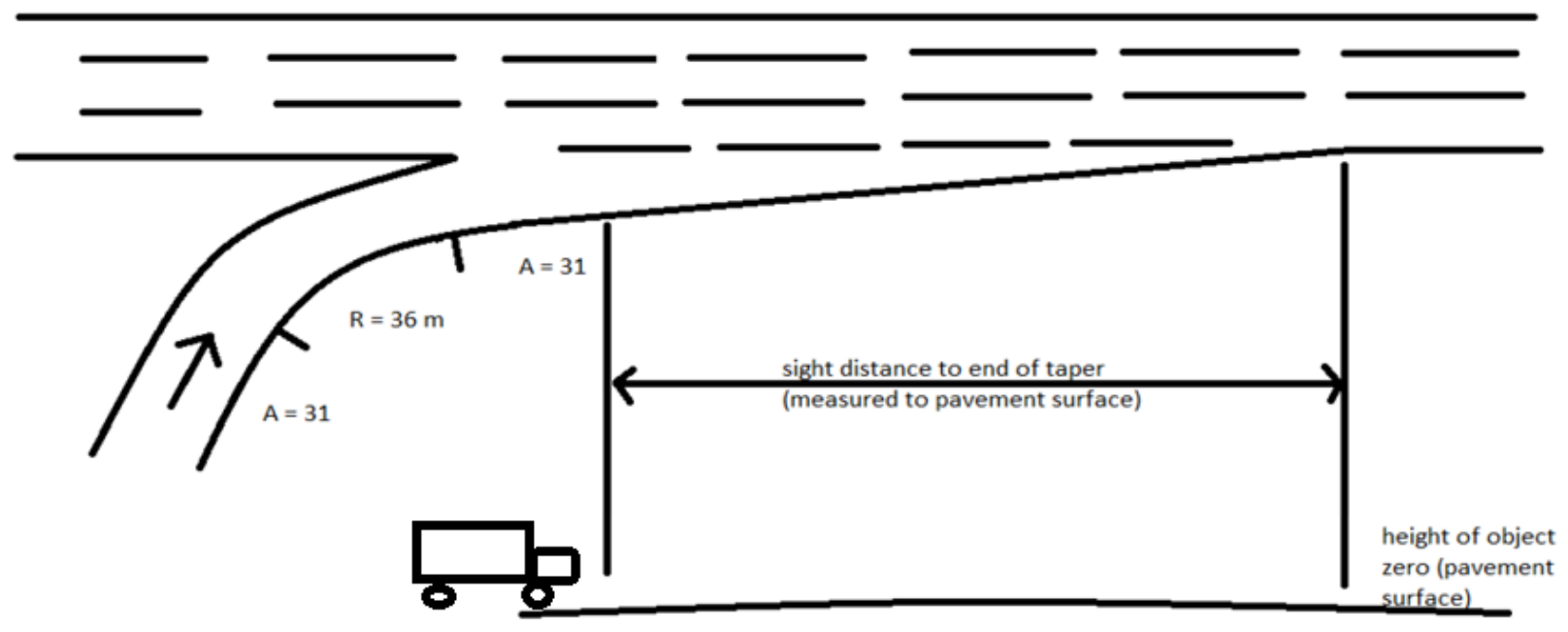

Figure 56. Curve radius and sight distance at the entrance ramp of an interchange. (Source: Adapted from TRB, 2012)

Maps with fishnet or hexagon polygons generated by hot spot analyses can be combined with road system shapefiles to determine the location and type of roads in hot spot areas. Investigations into the improvement of the design of various road types designated as hot spots such as interchanges can then be undertaken. For instance, if the lengths of the radius of the curves at most of the hot spot interchange ramps are less than the average length, then increases in the curve radius might lead to a reduction in the number of collisions. Other data such as the traffic volume of trucks at hot spot interchanges can be included to investigate other reasons for high collision rates. If traffic volume seems more closely related to the high incidence of collisions than geometric design parameters, 
ways to reduce traffic can be recommended. The reduction of traffic volume and the lengthening of the curve radius are both recommended as potential spill prevention measures that stem from hot spot analyses.

The optimized hot spot analysis technique used for mobile or transportation-related spills is a prevention planning tool for identifying areas that may require modifications to the road design. It is used as a preliminary step in location analysis to prioritize prevention measures. Statistically significant hot spot areas should be prioritized over cold spots while taking into account the overall spatial distribution of spills as carried out in regards to Figure 14. Although the central part of downtown Toronto was the only visible hot spot in Figure 14, the historical transportation-related spills were fairly, evenly distributed throughout the city which led to the investigation regarding highway spills. General guidelines for prevention planning tools for mobile spills include education BMPs such as anti-littering campaigns and adopt-a-highway initiatives (TRB, 2012). Such programs are intended to promote change in public perception and behaviour towards environmental issues (TRB, 2012). Source control BMPs that can be applied as preventative measures include street sweeping, highway litter cleanup, and catch basin stenciling. Litter and debris on highways and streets have often punctured the fuel tanks of transport trucks and cars, leading to numerous transportation-related oil spills. A high level of cleanliness of the transport system is therefore a preventative planning tool. A high level of truck or automotive maintenance, proper handling of transported oil, and a reduction in driver fatigue are other preventative planning tools for mobile spills. 


\subsubsection{Transportation-Related Model Planning Tools for Spill Control}

The control and treatment of oil spilled on highways and roads can be achieved by implementing oil-water separation retrofits. Unlike catch basin inserts, oil-water separation retrofits can be applied to "ultra-urban highways". Ultra-urban highways are defined as highway segments located in highly urbanized areas with limited opportunity for expansion of highway infrastructure. This limited opportunity for expansion is due to a lack of available surface space in the right-of-way (ROW) corridor for situating retrofit BMPs and high surrounding land costs (TRB, 2012). Oil-water or oil-grit separators are well-suited for ultra-urban highway retrofits because they are typically installed underground and have small space requirements (TRB, 2012).

Since the majority of highway spills have been found to occur at interchanges, oil-water separators should be retrofitted at major Toronto highway interchanges with oil spills, as displayed in Figure 15. Each circumstance in the following list of scenarios qualifies for the application of oil-water separator retrofits to ultra-urban highways (TRB, 2012):

- Oil-water separators are intended as hazardous materials traps or spill protection measures in areas with significant loadings of free-phase oils.

- Restrictive space constraints exist.

- Oil-water separators are used as pretreatment for other BMPs.

- Adequate maintenance capabilities exist and maintenance access is available (TRB, 2012). 
The first two circumstances in the list can be applied to major Toronto highway interchanges, therefore demonstrating the applicability of oil-water separator retrofits to these kinds of locations.

\subsubsection{Transportation-Related Model Planning Tools for Spill Response and} Clean-Up

The retrofitting of oil-water separators at major highway interchanges was developed as a mobile spill planning tool for spill control based on exploratory analyses between mobile spill occurrences and location type. Spill clean-up kits can be placed at or near oil-water separator locations for quick accessibility in the event of a transportation-related spill occurrence.

Additionally, interchanges and cloverleafs usually have vacant areas or large areas of managed landscapes, woods, or grass outside of the safety zones where non-structural and structural BMPs can be installed as retrofits. An example of the placement or installation of spill clean-up kits inside the quadrants of a cloverleaf interchange is shown in the sketch of Figure 57. 


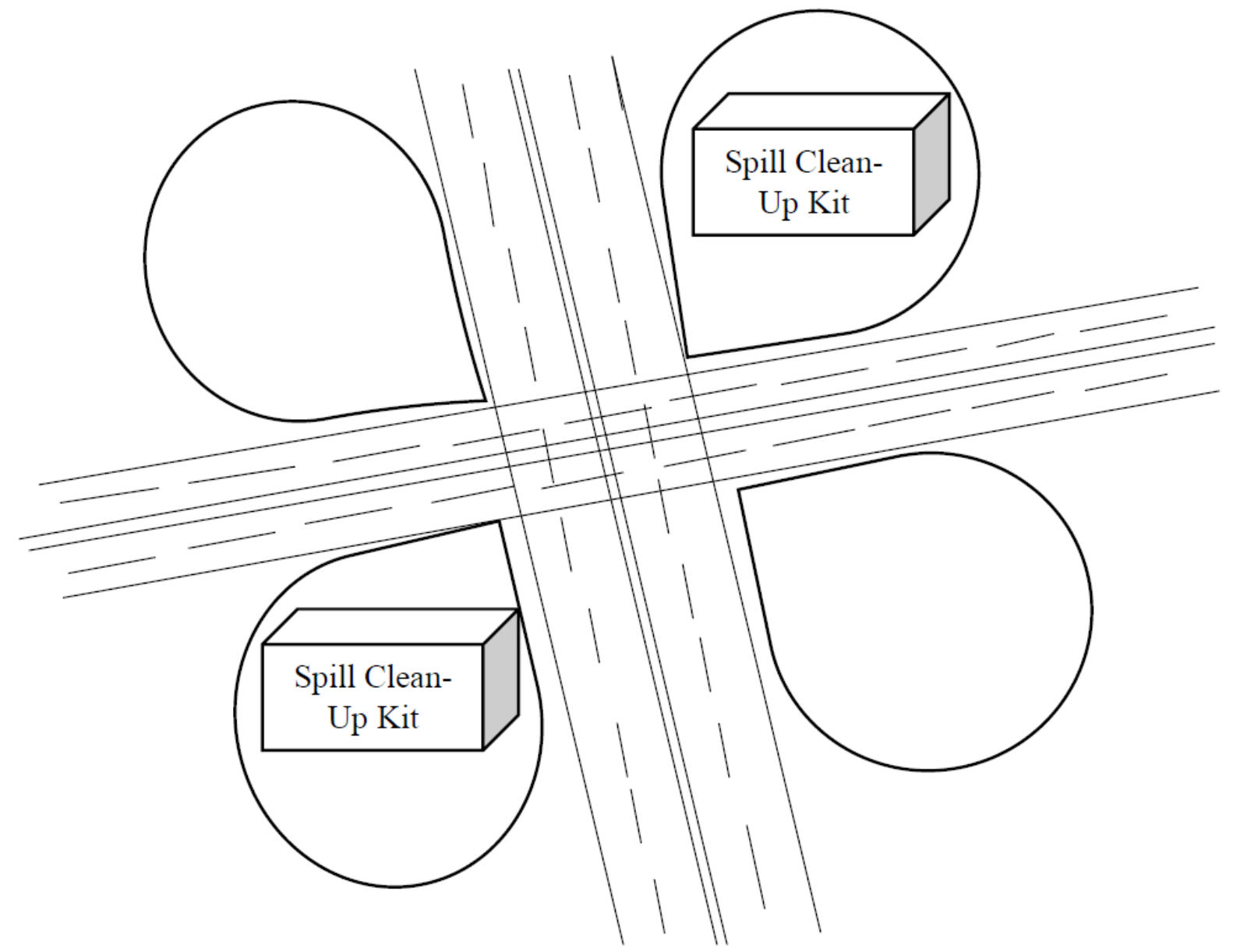

Figure 57. A sketch of a cloverleaf interchange with spill clean-up kits placed inside each quadrant.

In a retrofit project of a cloverleaf interchange, for example, trees were planted as a non-structural technique and bioretention cells were constructed as a structural technique inside the quadrants of the interchange (TRB, 2012).

The placement of spill clean-up kits inside the vacant areas within quadrants of cloverleaf interchanges also forms a spill response and clean-up planning tool for mobile or transportation-related spills. The spill clean-up kits could also be stored underground in 
these areas with an opening at ground level to minimize interference with structural and/or non-structural above-ground BMPs. The methodologies and results presented can be used to prioritize the placement of oil-water separators and spill clean-up kits based on the number of spills that have taken place at the interchanges. Interchanges with historically high amounts of spills should be prioritized over interchanges with historically low amounts of spills in terms of the installation or retrofitting of oil-water separators and the placement of spill clean-up kits. 


\section{Chapter 6: Conclusions and Recommendations}

The overall contribution to research of this study is the development of a comprehensive, three-stage spill management planning framework specifically for land-based oil spills. The three-stage spill management planning framework can be applied by municipalities which generally do not include all three stages of prevention, control, and response and clean-up as part of their spill management strategies to address land-based or inland oil spills.

Three key models were developed to generate quantitative planning tools for each of the three stages of spill management for each category of spill type (stationary and mobile):

1) A macro simulation program to predict future spill scenarios.

2) A spill path and travel-time model to delineate the entire spill path of an inland spill and calculate the total travel-time ( $\mathrm{min})$.

3) A transportation-related highway spill model.

Each of the three models is a contribution to research. Using these models, specific suggestions or policy guidelines were exemplified for the two case study areas. This approach can be followed by municipalities to develop their own spill management strategies.

The suggestions developed for the case study areas are viewed as planning tools that have been derived from the models. Generic planning tools have been developed from the main inland oil spill database encompassing historical data for both stationary and transportation-related spills. However, planning tools specific to each of these spill types 
have been developed using historical data as well. Statistical analyses of historical spill data can therefore help decision makers to manage spills as demonstrated, for example, by the development of prevention planning tools for the stationary spill sub-database using historical data. Planning tools developed for all three stages of prevention, control, and response and clean-up of transportation-related spills are based on historical transportation-related spill data.

The macro program simulated future spill data for stationary inland oil spills to develop planning tools such as the sizing of oil-water separators to retrofit existing oil-water separators or to install new oil-water separators in the future. The sizing of the oil-water separators was based on the aggregate output data of predicted spill simulations. In the past, oil-water separators were sized based only on historical data. Future spill data can now be used to determine the capacity of oil-water separators that may be required.

The simulated future spill data has been displayed in ArcGIS and hot spot analyses or other mapping clustering techniques and clustering methods can be used to specify where the highest concentrations of spills will occur in the future. Areas of high concentrations of spills can be verified with hot spot analyses to be statistically significant and plans for the installation or retrofitting of control devices can be made for these areas. The hot spot areas can be identified as future spill prone areas and serve as high-priority areas in terms of applying spill management options. The delineation of high-priority spill prone areas can be used to apply planning tools from all three stages of spill management. 
The transportation-related spill sub-database can be similarly applied in future research work to simulate future transportation-related spill data. Historical transportation-related spill data was used to perform spatial analyses using hot spot analysis (optimized version). The results were analyzed to further investigate transportation-related spills. The highway spill model was developed to propose strategies for spill prevention, control, and response and clean-up for highway interchange locations which are spill prone locations in terms of historically occurring highway spills. For example, spill clean-up kits can be placed in the quadrants of cloverleaf interchanges or partial cloverleaf interchanges to be easily accessible when spill events occur in these areas.

Predicted transportation-related spill data can be used in a similar manner as for predicted stationary spill data. Spill prone areas can be confirmed to be hot spots which can be used to prioritize the placement of spill control devices, and clean-up supplies and equipment. The accuracy of the predicted transportation-related spill data can be investigated to determine whether the predicted spill points should truly be associated with the hot spots. More specific and accurate address data for transportation-related spills will be required when recommending alterations to the design of highways or roadways which was suggested as a prevention planning tool. Future transportation-related spill analysis should also focus on non-highway spills The accuracy of the macro simulation model in terms of the accuracy of the historical data can be investigated for transportation-related spills as well as stationary spills. The uncertainty of the predicted values of Spill Mass ( $\mathrm{kg}$ ), Interevent Time (days) and 
$\%$ Clean-Up can be determined to ascertain whether the uncertainty of the predicted values is limited by the accuracy of the historical data sets. The macro program can also be refined to only predict spills within areas where spills are feasible. For example, an inland oil spill should not originate in the middle of a waterway. Although future predicted spill locations are usually reflective of past spill locations, predicted future spill points should be as realistic as possible.

Recommendations have also been made with respect to improving the system of inland oil spill data collection. More accurate address data for the site of the spill event has been recommended by using GPS coordinates. More detail regarding the exact location of the spill event can also be relayed by requesting the caller to clarify whether the address given is the address of the spill event or the address of the location which has placed the call to report the spill.

Future research should investigate the impacts of inland oil spills on soil environments since there may be some absorption by soils along the overland flow path of the spill, even in urban environments. The absorption of spilled oil by soils may lead to negative impacts on the groundwater which could then permeate into surface water environments. The spill data must be filtered for impacts on soils, vegetation and groundwater which indicate that some absorption may have occurred. The methodology developed can then be followed for the data set to develop planning tools specific to these environments.

The collection of future spill data should entail the identification of rain or dry weather conditions at the time and place of the spill event. Other meteorological conditions and 
spill characteristics which will help to model the spill before it enters the storm sewer network are: wind speed, air temperature and the physical and chemical characteristics of the spilled oil. These parameters will help to calculate the evaporative loss of the oil as it travels over the land (Farrar et al., 2014).

Land cover type or land use type can be used to calculate the different rates of oil loss as oil moves across the surface. In future work, Manning's $n$ for an urban area can be calculated by using a weighted average based on the proportion of various surface types or land use types in the study area and the value of Manning's $n$ for each surface. The built landscape, as opposed to just the natural terrain, could be included in the spill path and travel-time model to provide a more realistic spill path. The release rate of the spilled oil would also help to model the spill and is sometimes provided in the summary/report sections of the current spill data sets (Farrar et al., 2014).

The spill path and travel-time model assumes access points or sewer receptacles as the point of entry into the storm sewer network which is sufficient. However, a more realistic model could be developed by using the stormwater inlets as the points of entry of the storm sewer system. The GIS software program imposed certain limitations which complicated the development of the spill path and travel-time model. For example, there was no available tool or function to calculate the slope of a line. If this limitation could be overcome, the spill path and travel-time model could be shortened and simplified. Lastly, future work could expand upon the simple Web-based GIS prototype that was developed to merge the prototype with the spill path and travel-time model. 


\section{Appendices}

Appendix A.1: Spill Path and Travel-Time Model Displayed in Three Main Parts

Part 1: Generation of Cost Distance Output and Output Polyline Shapefile

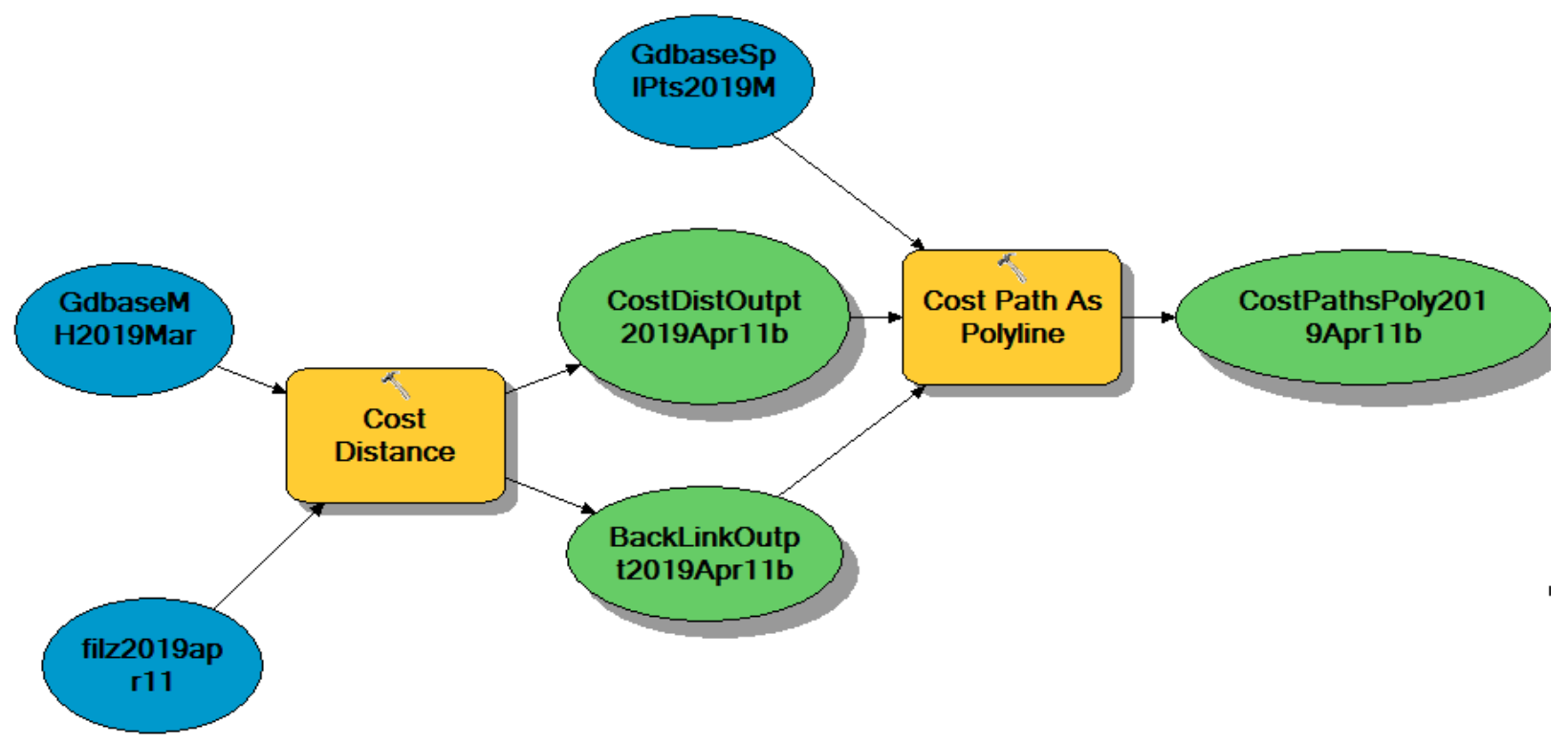

Part 2: Modification of Output Polyline Shapefile

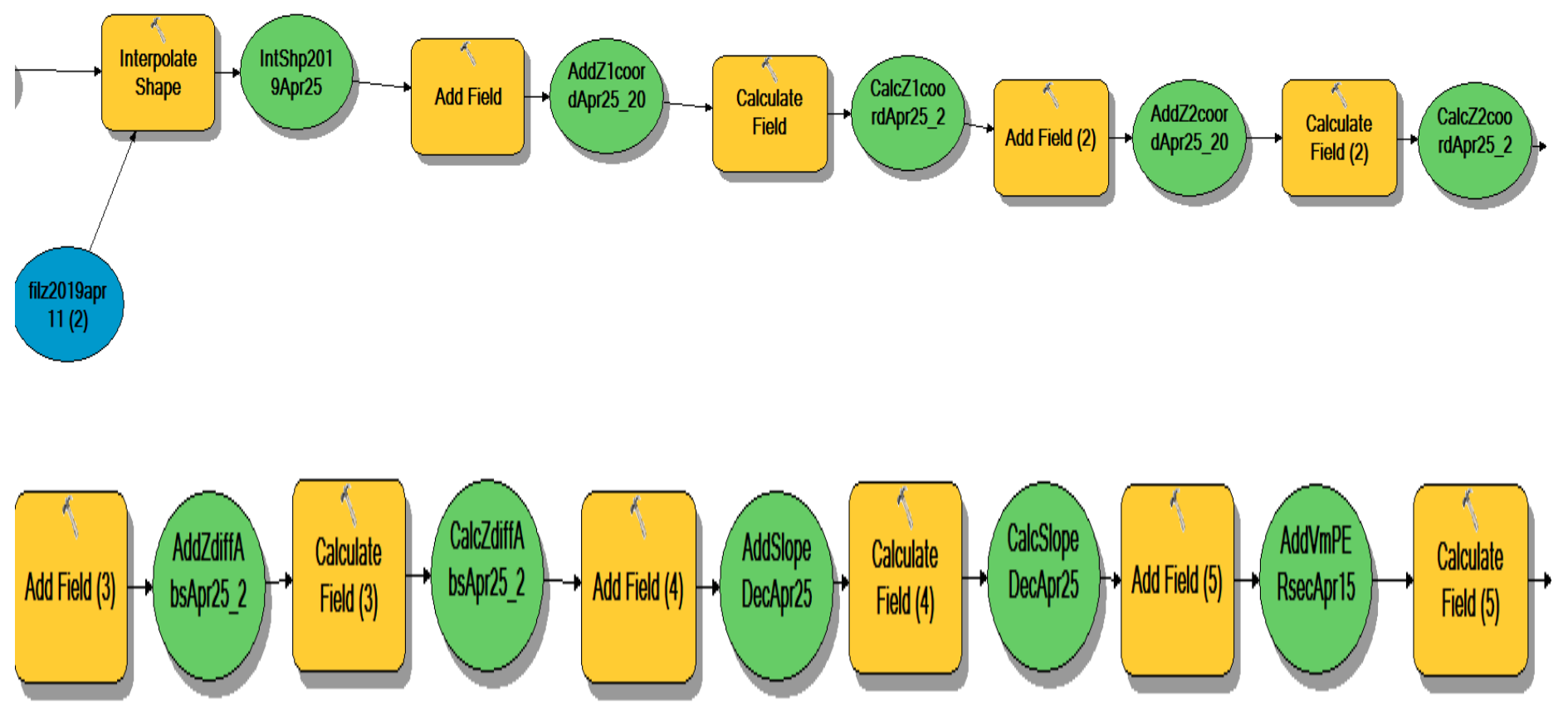


Appendix A.1 (cont'd): Spill Path and Travel-Time Model Displayed in Three Main Parts

Part 2 (cont'd): Modification of Output Polyline Shapefile

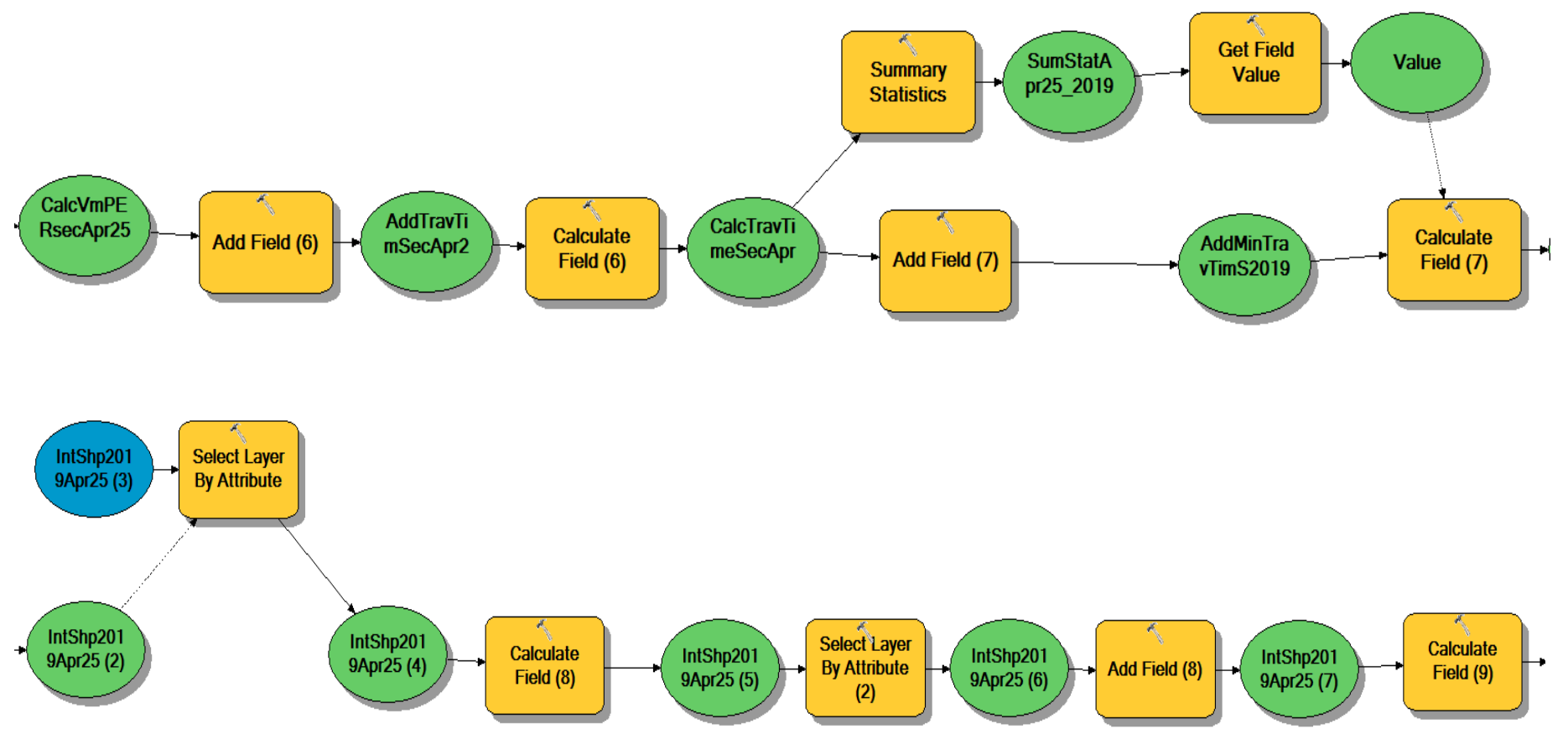

Part 3: Creation and Development of Geometric Network

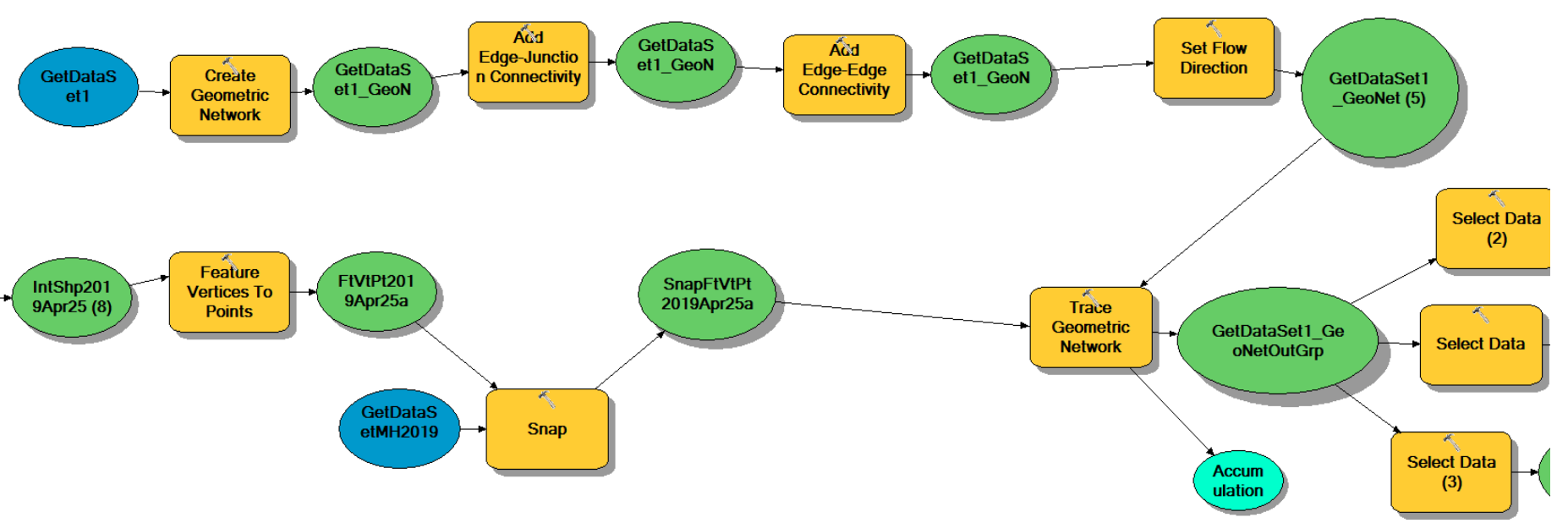


Appendix A.1 (cont'd): Spill Path and Travel-Time Model Displayed in Three Main Parts

$\underline{\text { Part } 3 \text { (cont'd): Creation and Development of Geometric Network }}$

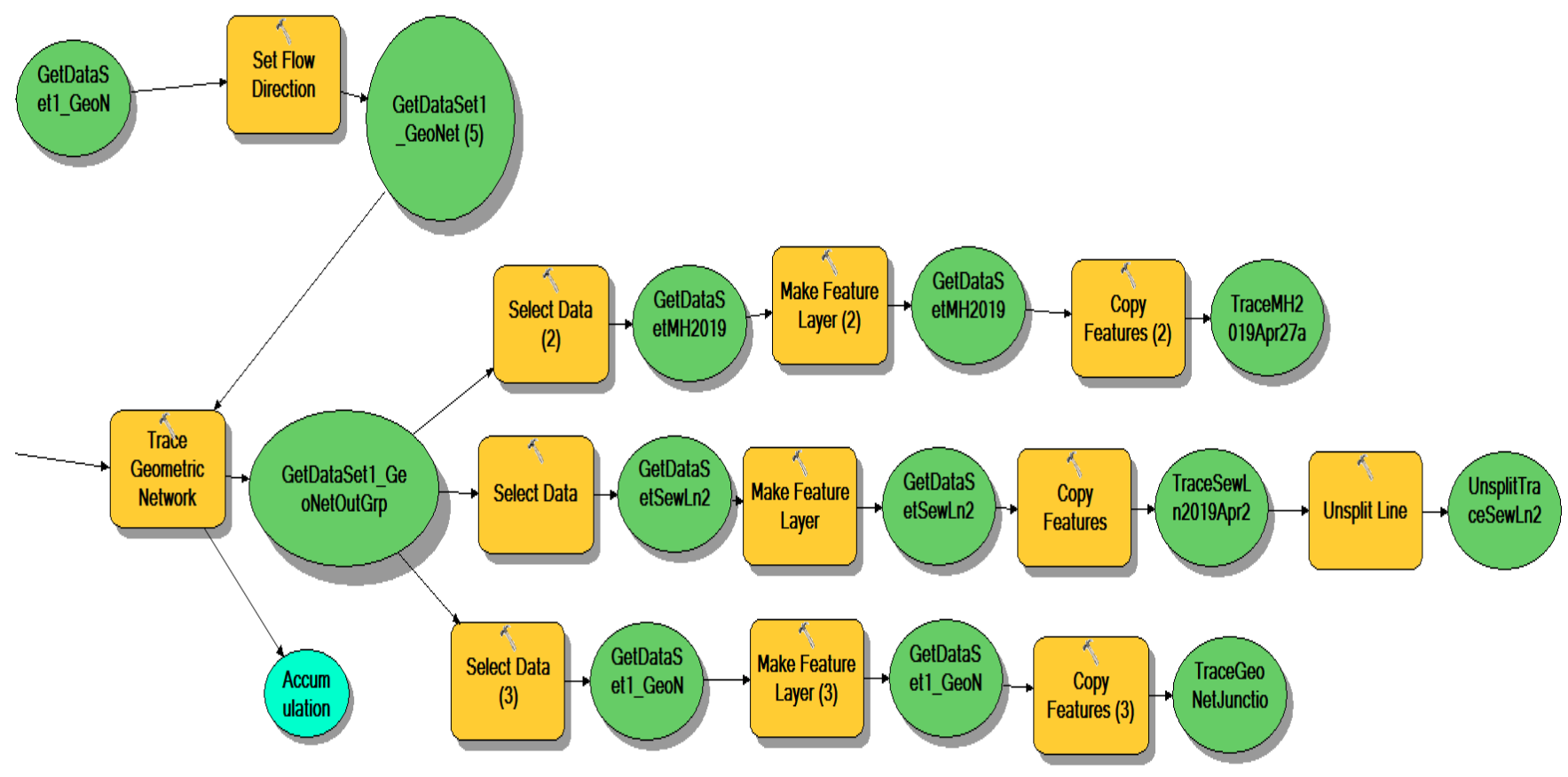




\section{Appendix B.1: Types of Environments Impacted by Inland Oil Spills in Ontario}

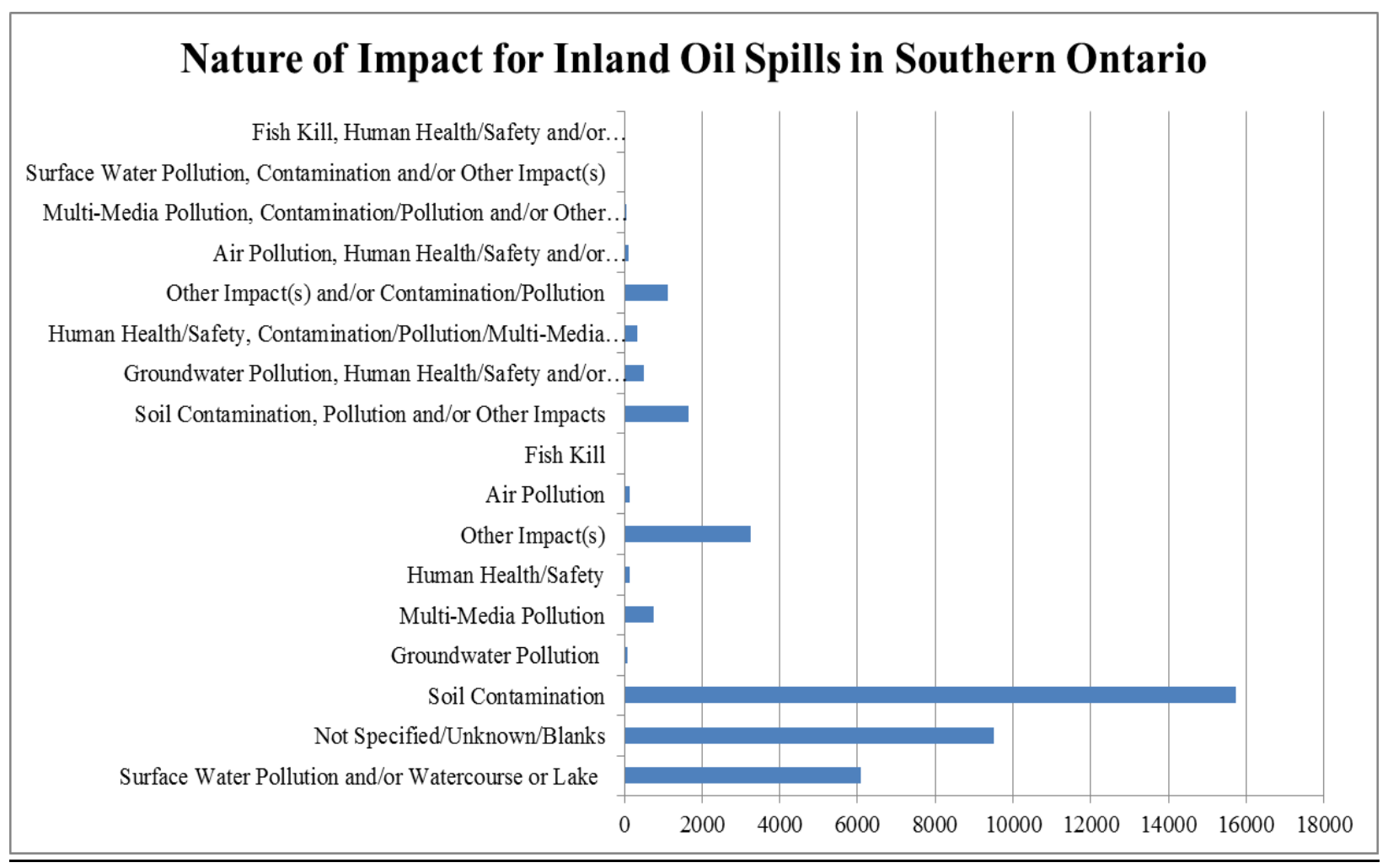


Appendix C.1: A Map Service of Transportation-Related Spills With Other Data

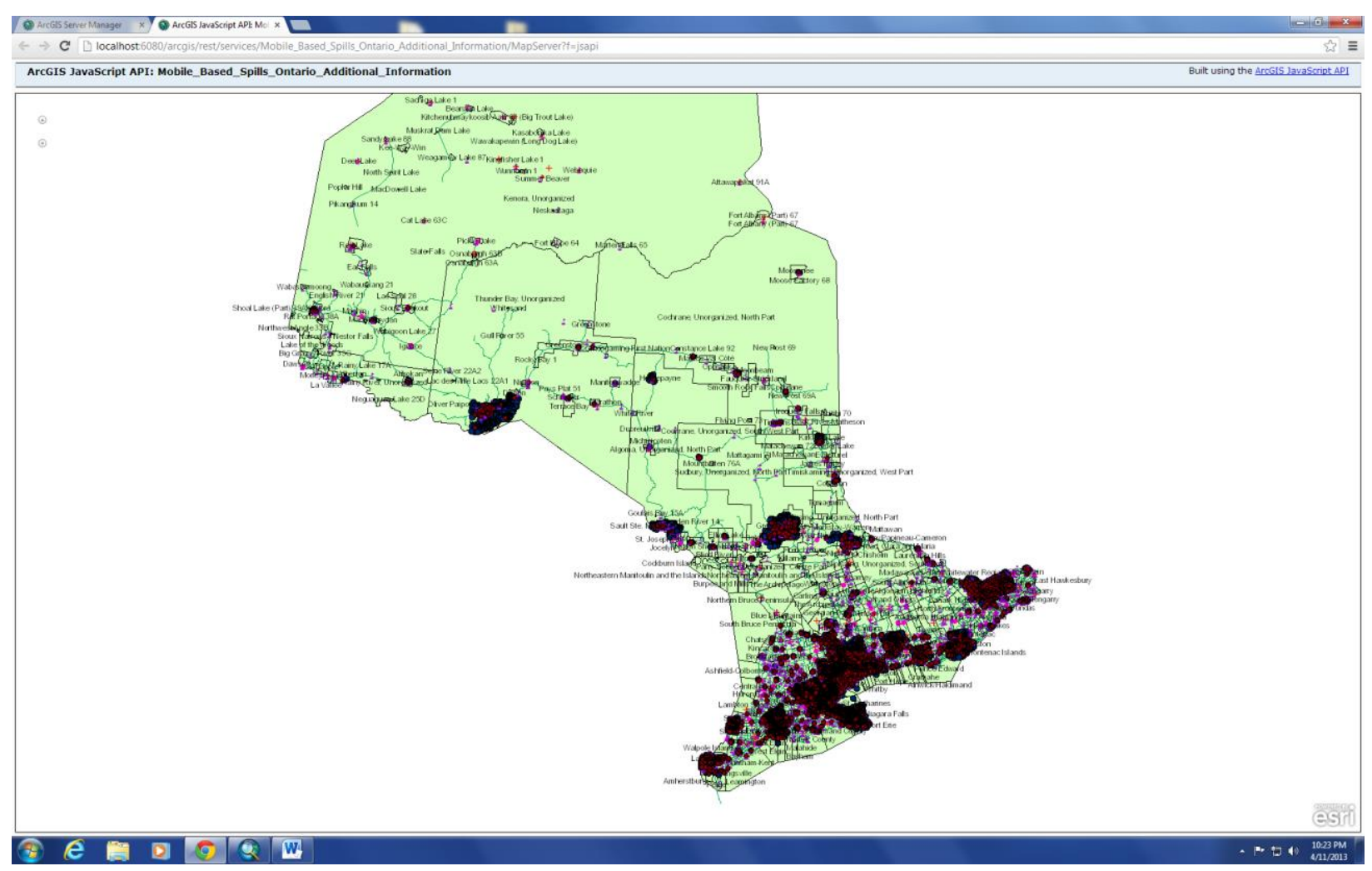




\section{References}

Acts. (2013, February). Retrieved December 15, 2013, from

http://www.ec.gc.ca/default.asp?lang=En\&n=E826924C-1

Ahmed, S.E, Beaver, B.M., Beaver, R.J. \& Mendenhall, W. (2014). Introduction to Probability \& Statistics, Third Edition. United States: Nelson Education Ltd.

A Survey and Clarification of DEM Vertical Accuracy. (2019). Retrieved September 7, 2019 from https://www.bigdataearth.com/elevation/dem-vertical-accuracy/

Awawdeh, M., Cherian, G., Chudiwale, V., Fan, G., Rao, M., and Thomas, J. (2007). A web-based GIS Decision Support System for managing and planning USDA's Conservation Reserve Program. Environmental Modelling \& Software, 22, 1270 1280.

Bloom, S.A., Grunwald, S., Reddy, K.R., and Mathiyalagan, V. (2004). A WebGIS and geodatabase for Florida's wetlands. Computers and electronics in agriculture. 17.

Cao, W. (2013). Probabilistic Spill Occurrence Simulations and Quantitative Water Quality Risk Analysis (Doctoral dissertation, Ryerson University, 2013

Castaldelli, G., Dezfuli, B.S., Giari, L., \& Lanzoni, M. (2012). The impact of an oil spill on organs of bream Abramis Brama in the Po River. Ecotoxicology and Environmental Safety, 77, 18-27.

Cheng, V. (2003). The Development of Risk-Based Spill Management Criteria Related to the Beneficial Use Impairments in the St. Clair River. (Master's dissertation, Ryerson University, 2002). 
Chin, D.A. (2013). Water-Resources Engineering, Third Edition. Upper Saddle River, NJ: Pearson Education, Inc.

Chui, J. K. L. (2002). Control of Oil Spills in Urban Areas (Master's dissertation, Ryerson University, 2002).

Cooper, A. \& Johnson, C. (2018, May 9). Now near 100 million bpd, when will oil demand peak? Retrieved January 10, 2019, from https://www.reuters.com/article/us-oil-demand-peak/now-near-100-million-bpdwhen-will-oil-demand-peak-idUSKCN1M01TC

Emergency Planning. (2012, April). Retrieved December 22, 2013, from http://www.ene.gov.on.ca/environment/en/about/emergency_planning/STD01_078 384.html

Environmental Emergencies. (2013, March). Retrieved December 22, 2013 from Environment and Climate Change Canada, http://www.ec.gc.ca/eeue/default.asp?lang=En\&n=8A6C8F31-1

Environmental Systems Research Institute [ESRI]. (2017). ArcGIS Help Library [Software manual]. Redlands, California.

Environmental Systems Research Institute [ESRI]. (2013). ArcGIS 10.1 for Server (Windows) Help, California, U.S.A.

Farrar, W., Galagan C., Isaji, T., \& Knee, K. (2014, June 5). GIS Technology Applied to Modeling Oil Spills on Land. [Electronic version].

Fingas, M. (Ed.), Brown, C., Challenger, G., Etkin, D., Hollebone, B., Kirkby, M., et al. (2011). Oil spill science and technology: prevention, response and clean up. Massachusetts: Elsevier. 
Han, H. Y. (2007). A Web-Based GIS Planning Framework for Urban Oil Spill Management (Master's dissertation, Ryerson University, 2007).

Hennighausen, A. (2012, July). Enbridge’s Quiet $\$ 765$ Million Oil Spill. Retrieved March 5, 2014, from http://www.bloomberg.com/slideshow/2012-07-25/enbridges-quiet-765-million-oil-spill.html

Hunter, Justine (2018, September 15). Trans Mountain pipeline delay means gaps in oil spill response remain. Retrieved March 21, 2019, from https://www.theglobeandmail.com/canada/british-columbia/article-transmountain-pipeline-delay-means-gaps-in-oil-spill-response-remain/

Klerks, P.L. , Nyman, J.A., \& Bhattacharyya, S. (2004). Relationship between hydrocarbon measurements and toxicity to a chironomid, fish larva and daphna oils and oil spill chemical treatments in laboratory freshwater marsh microcosms. Environmental Pollution, 129, 345-353.

Li, J. (2002). Spill Management for the Toronto AOC: The City of Vaughan Study. Toronto, Ontario.

Li, J. (2005). Urban Spill Management Planning in the Greater Toronto Area. Environmental Informatics Archives, 3, 67-75.

McDiarmid, J. (2013, October). Enbridge oil sands pipeline plan raises chilling issues for GTA. Retrieved March 10, 2014, from, http://www.thestar.com/news/gta/2013/10/02/enbridge_oilsands_pipeline_plan_rai ses_chilling_issues_for_gta.html 
McDuffie, E., Mallari, N., Manrique-Zedar, L., Pate, D., \& Smith B. (2015, April). A Study of Ecosystem Services Provided by a Stormwater Retrofit on a Public School Campus in Orange County, North Carolina. Sustainability: The Journal of Record, 8(2).

Michel, J., Etkin, D.S., Gilbert, T., Urban, R., Waldron, J., \& Blocksidge, C.T. (2005). Potentially Polluting Wrecks in Marine Waters. In 2005 International Oil Spill Conference Issue Paper (pp.76).

Milanelli, J.C.C., Pincinato, F.L., and Riedel, P.S. (2009). Modelling an expert GIS system based on knowledge to evaluate oil spill environmental sensitivity. Ocean \& Coastal Management, 52, 479-486.

Northwest Territories Environment and Natural Resources. (2014, August). Hazardous Materials Spill Database. Retrieved August 24, 2014, from http://www.enr.gov.nt.ca/programs/hazardous-materials-spills/hazardousmaterials-spill-database

Oberholster, P.J., Blaise, C., \& Botha, A.M. (2010). Phytobenthos and phytoplankton community changes upon exposure to a sunflower oil spill in a South African protected freshwater wetland [Electronic version]. Ecotoxicology, 19, 1426-1439.

Ontario Ministry of the Environment. (1994, April). Spills Action Centre Operations. Retrieved August 21, 2013, from Ontario Ministry of the Environment Web site: http://www.ene.gov.on.ca/environment/en/resources/STD01_076609.html

Ontario Ministry of the Environment and Climate Change. (2013, December). Spills Action Centre. Retrieved December 28, 2013 from http://www.ene.gov.on.ca/environment/en/about/emergency_planning/STDPROD _080741 
Owens, E.H. (2002). Response Strategies for Spills on Land. Spill Science \& Technology Bulletin, 7(3-4), 115-117.

Pollution Prevention. (2013, October). Retrieved December 18, 2013, from http://www.ec.gc.ca/p2/

Pollution Prevention Planning. (2013, October). Retrieved December 18, 2013, from http://www.ec.gc.ca/planp2-p2plan/default.asp?lang=En\&n=F7B45BF5Saba, V.S. \& Spotila, S.R. (2003, May). Survival and behavior of freshwater turtles after rehabilitation from an oil spill. Environmental Pollution, 126, 213223.

Saba, V.S. \& Spotila, S.R. (2003, May). Survival and behavior of freshwater turtles after rehabilitation from an oil spill. Environmental Pollution, 126, 213-223.

Schardt, J. (2010). Amending the Great Lakes Quality Agreement. Retrieved August 26, 2013, from http://binational.net/glwqa_2010_e.html

Shell Petroleum Development Company of Nigeria Ltd. (2014, November). Oil Spill Data. Retrieved November 26, 2014 from http://www.shell.com.ng/environment society/environment-tpkg/oil-spills.html (accessed 26 November 2014)

Spills Action Centre, Regional Operations Division, Ministry of Environment and Energy. (1993, November). Spills Action Centre Summary Report of 1992 Spills (Technical Publication ISSN 1192-5078). Queen's Printer for Ontario, 1993.

Spills Action Centre. (2012, November). Retrieved December 27, 2013, from http://www.ene.gov.on.ca/environment/en/about/emergency_planning/STDPROD _080741 
Tang, N. M. K. (2005). A Land-Based Oil Spill Management Planning Framework for the Petroleum Industry (Master's dissertation, Ryerson University, 2005).

TIBCO Software Inc. [StatSoft] (2017). Electronic Statistics Textbook [Software Manual]. Palo Alto, California.

Tranforming an Oregon Watershed, Once Marred by a Gasoline Spill, into Fish-Friendly Habitat. (2013). Retrieved December 27, 2013, from National Oceanic and Atmospheric Administration Website:

http://response.restoration.noaa.gov/about/media/transforming-oregon-watershedonce-marred-gasoline-spill-fish-friendly-habitat.html

Transportation Association of Canada [TAC]. (2007). Geometric Design Guide for Canadian Roads. Ottawa, Canada: Author.

Transportation Research Board [TRB]. (2012). National Cooperative Highway Research Program Report 728 Guidelines for Selecting Modifications to Existing Roadway Drainage Infrastructure to Improve Water Quality in Ultra-Urban Areas. Washington, DC: U.S.

Van Dongen, M. (2018, June 15). Imperial Oil to replace jet fuel pipeline from Waterdown to Toronto. Retrieved January 21, 2019 from https://www.thespec.com/news-story/8672520-imperial-oil-to-replace-jet-fuelpipeline-from-waterdown-to-toronto/

Weigh Anchor. What the Trans Mountain pipeline will mean for B.C.'s Coast. (2018). Retrieved April 30, 2019, from https://www.theglobeandmail.com/news/britishcolumbia/kinder-morgan-trans-mountain-pipeline-bc-coast/article35043172/ 
Wildlife Rescue. (2013). Retrieved December 21, 2013, from The Toronto Centre Website: http://torontowildlifecentre.com/oil-spill-response

Young, L. (2014, August). Open Data: Alberta oil spills 1975-2013. Retrieved August 21, 2014, from http://globalnews.ca/news/622513/open-data-alberta-oil-spills$1975-2013 /$ 